\title{
STRUCTURE AND FUNCTION OF A.NIDULANS PSI FACTOR PRODUCING OXYGENASE A
}

\author{
Dissertation \\ zur Erlangung des mathematisch-naturwissenschaftlichen Doktorgrades \\ „Doctor rerum naturalium” \\ der Georg-August-Universität Goettingen
}

vorgelegt von

Christian Koch

aus Mühlhausen 
Die vorliegende Arbeit wurde in der Zeit von Januar 2010 bis August 2012 am Albrecht-vonHaller-Institut für Pflanzenwissenschaften der Georg-August Universität unter der Anleitung von Prof. Dr. Ivo Feußner angefertigt.

Mitglied des Betreuungsausschusses/ Erstreferent: Prof. Dr. Ivo Feußner

Abteilung Biochemie der Pflanze, Albrecht-von-Haller-Institut, Georg-August Universität Göttingen

Mitglied des Betreuungsausschusses/ Zweitreferentin: Prof. Dr. Marina Bennati

Arbeitsgruppe für EPR-Spektroskopie, Max Planck Institut für biophysikalische Chemie, Göttingen

Mitglied des Betreuungsausschusses: Prof. Dr. Kai Tittmann

Abteilung Bioanalytik, Albrecht-von-Haller-Institut, Georg-August Universität Göttingen

Mitglieder der Prüfungskommission:

Prof Dr. Ulf Diederichsen

Institut für Organische und Biomolekulare Chemie, Georg-August Universität Göttingen

Prof. Dr. Ralf Ficner

Abteilung für Molekulare Strukturbiologie, Institut für Mikrobiologie und Genetik, Georg-August

Universität Göttingen

Prof. Dr. Heinz Neumann

Angewandte Synthetische Biologie, Institut für Mikrobiologie und Genetik, Georg-August Universität Göttingen

Tag der mündlichen Prüfung: 01.Oktober 2012 


\section{Affidavit}

Herewith, I declare that the present thesis was written on my own without other sources and aids than quoted.

Goettingen, August 2012

Christian Koch 



\section{STRUCTURE AND FUNCTION OF A. NIDULANS PSI FACTOR PRODUCING OXYGENASE $A$}





\section{TABLE OF CONTENTS}

1 Introduction

1.1 PpoA: A tale of green molds and red enzymes ................................................................ 11

1.2 Involvement of oxylipins in host-pathogen interaction..................................................... 12

1.2.1 Fungal infections and mycotoxin production-A curse for agriculture .........................13

1.2.2 The impact of oxylipins in general and Psi-factors in special on fungal pathogenicity.. 15

1.3 A mechanical view on PpoA, an fascinating enzyme to study heme-chemistry...................18

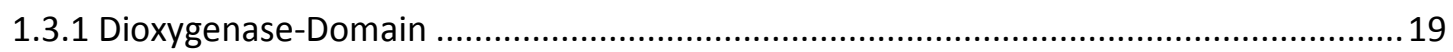

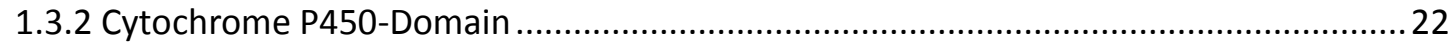

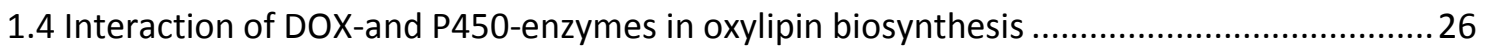

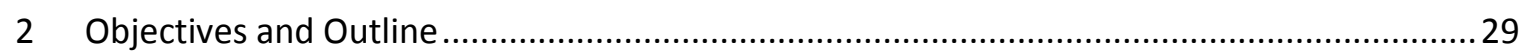

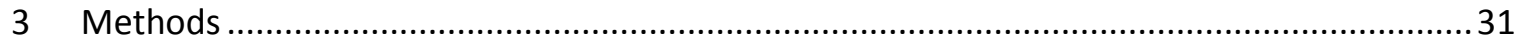

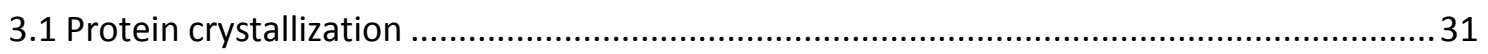

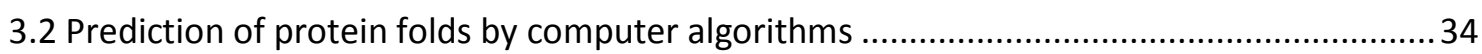

3.3 Characterizing the oligomeric assembly of a macromolecule by Small-angle X-ray scattering

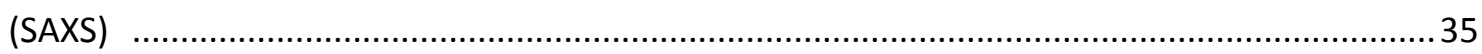

3.4 Electron Paramagnetic Resonance and Double Electron Electron Resonance......................37

3.4.1 Double electron-electron-resonance (DEER) ………................................................. 39

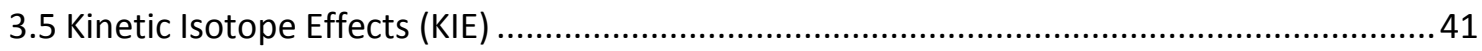

3.6 Measuring redoxpotentials by direct electro chemistry.................................................. 41

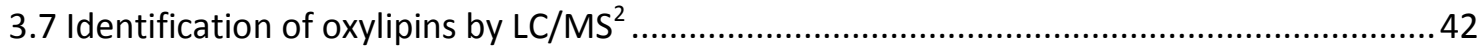

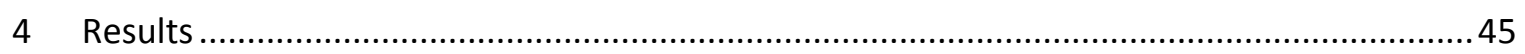

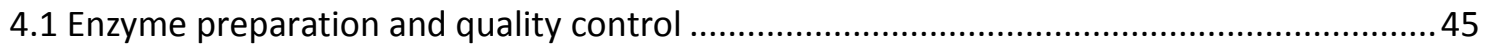

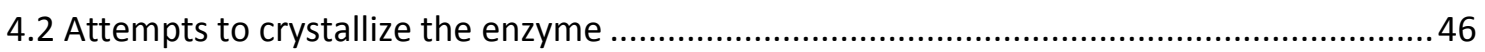

4.2.1 Refinement of a previously identified promising condition .......................................46

4.2.2 New screening for conditions eventually yielding crystals ........................................46

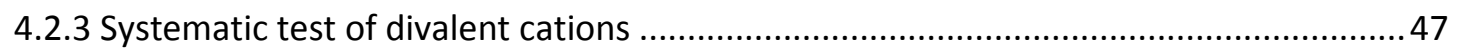

4.2.4 Tryptic digest and/or reductive lysine methylation of PpoA and subsequent crystal screens

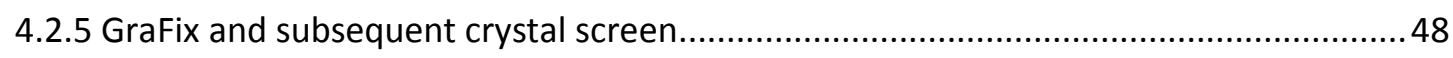

4.3 Template-based structure prediction .......................................................................... 51 
4.3.1 Predicted structure of PpoA's DOX-domain and identification of residues putatively involved in dioxygenation

4.3.2 Predicted structure of PpoA's P450-domain and identification of residues putatively involved in hydroperoxy fatty acid rearrangement 55

4.4 Validation of proposed amino acid determinants by biochemical characterization of respective variants 59

4.4.1 Substrate binding to PpoA's DOX-Domain may be governed by ionic interaction with $\operatorname{Arg} 336$ .59

4.4.2 Tyr327: A second tyrosine involved in hydrogen abstraction from the fatty acid substrate's $\mathrm{C}_{8}$ ? .61

4.4.3 Determinants of substrate binding to PpoA's hydroperoxide-isomerase-domain .63

4.4.4 Asparagine 887 is crucially involved in hydroperoxide rearrangement .71

4.5 Attempts to convert 5,8-LDS to 7,8-LDS . .74

4.6 Quaternary structure of PpoA .76

4.6.1 Measuring the native low-resolution structure of PpoA by SAXS.................................76

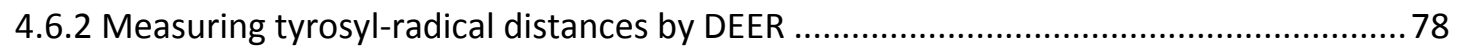

4.7 Kinetic isotope effects to probe rate-limiting steps of hydrogen abstraction.......................85

4.7.1 Utilized probes .85

4.7.2 Pseudo steady-state kinetics 85

4.7.3 End-point measurements. .89

4.7.4 Is the high KIE for dideuteration at $\mathrm{C}_{8}$ explainable by hydrogen tunneling? 90

4.7.5 Explaining the effect of dideuteration at $C_{5}$ on oxygen consumption 91

4.7.6 Stereochemistry of the dioxygenation, catalyzed by PpoA .94

4.8 Protein film voltammetry of PpoA .95

5 Discussion .97

5.1 Structure-Function-Relationship. .97

5.1.1 Active site structure of the DOX-domain and determinants of dioxygenase-activity ...98 5.1.2 Active site structure of the P450-domain and determinants of hydroperoxy fatty acid

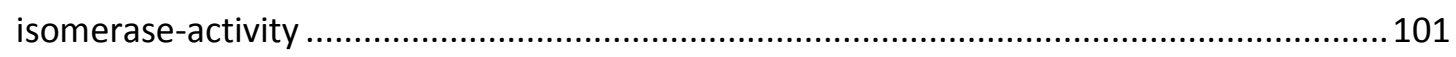

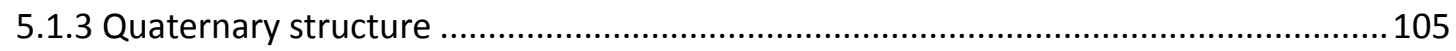

5.2 Kinetic isotope effects to probe the kinetic contribution of hydrogen-transfer steps........107

5.3 Electrochemistry of PpoA 


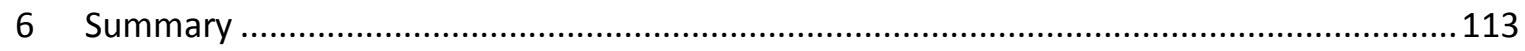

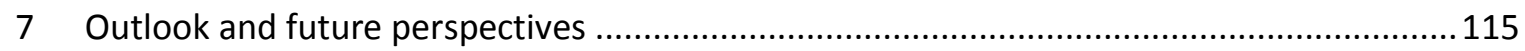

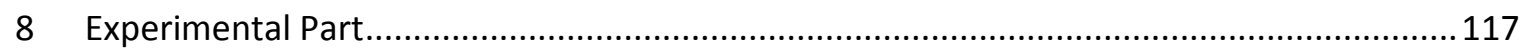

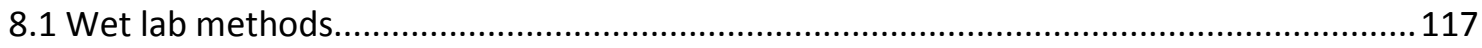

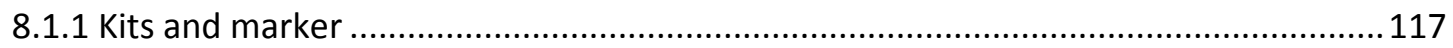

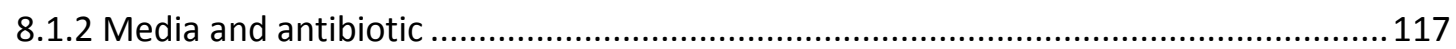

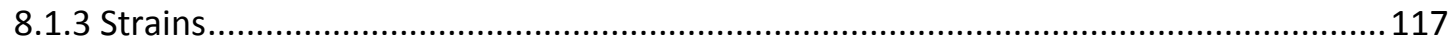

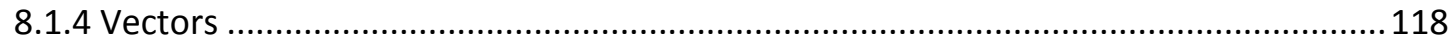

8.1.5 Preparation of competent E. coli for transformation by heat-shock ..........................118

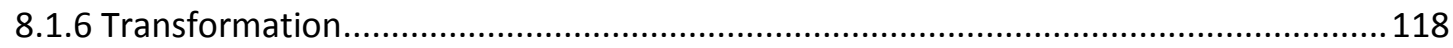

8.1.7 Plasmid-DNA-isolation from E. coli ........................................................................ 118

8.1.8 Site-directed mutagenesis by Polymerase-Chain-Reaction ........................................119

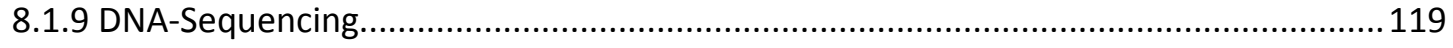

8.1.10 Expression of PpoA wild type and its variants in E. coli .............................................121

8.1.11 Cell disruption of E.coli expression cultures ........................................................ 121

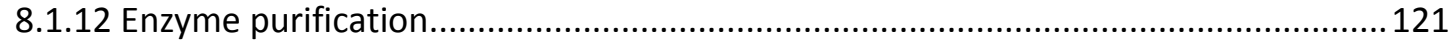

8.1.13 SDS-Polyacrylamide-Gel Elektrophoresis (SDS-PAGE)............................................. 121

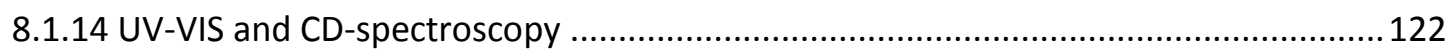

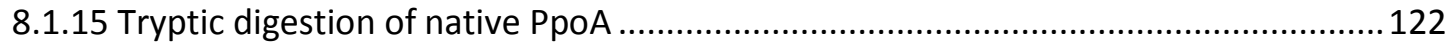

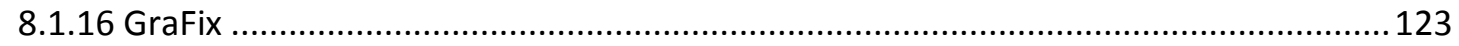

8.1.17 Reductive methylation of surface exposed lysines ................................................123

8.1.18 Measuring DOX-kinetics by monitoring depletion of dissolved oxygen .....................123

8.1.19 Synthesis of ${ }^{14} \mathrm{C}$-labeled 8-HPODE and 8-HPODE methyl ester..................................124

8.1.20 Substrate conversion and analysis of the product pattern by LC-MS ${ }^{2}$..................... 124

8.1.21 Measurement of tyrosyl radical distances by DEER ................................................... 124

8.1.22 Direct electrochemistry of PpoA within a surfactant film.......................................125

8.1.23 Imidazole titration and spectral binding assay .................................................... 125

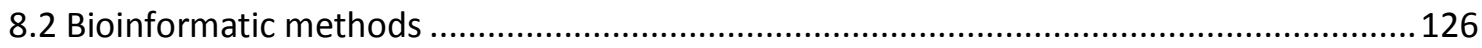

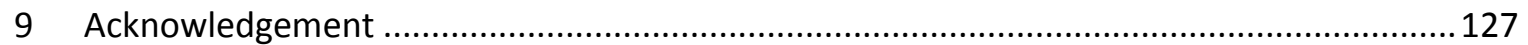

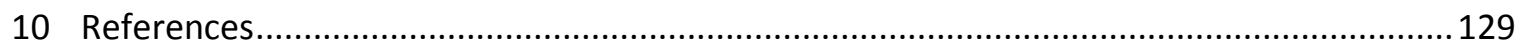

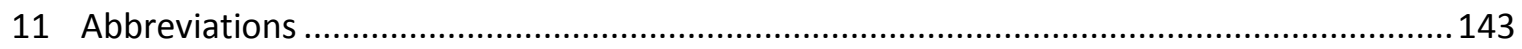





\section{INTRODUCTION}

\subsection{PpoA: A tale of green molds and red enzymes}

Oxidized fatty acids acting as signal molecules in mammals (Funk, 2001) and plants (Wasternack, 2007) are commonly termed oxylipins. In the late 1980's similar molecules have been identified in the ascomycete Aspergillus nidulans and were shown to be involved in developmental processes (S P Champe et al., 1987; S P Champe and el-Zayat, 1989; Mazur et al., 1991). By influencing the ratio of conidio- and ascospores these compounds obviously acted as well as hormone-like molecules and consequently have been named Precocious-Sexual-Inducers or, abbreviated, Psi-factors. Structurally, they are unsaturated $\mathrm{C}_{18}$ fatty acids with a hydroxyl-group at $\mathrm{C}_{8}$ and an optional hydroxyl group at $\mathrm{C}_{5}$. Almost in parallel it was established that Gaeumannomyces graminis, another ascomycete, produces similar hydroxylated derivatives of unsaturated $C_{18}$ fatty acids with a hydroxyl function at $C_{8}$ and a second, facultative one at $C_{7}$ (Brodowsky et al., 1992). Four years later the enzyme responsible for oxygen insertion at $C_{8}$ of the fatty acid was isolated from this fungus (Chao Su and Ernst H. Oliw, 1996). Although it seemed that the hydroperoxide-isomerase activity that rearranged this fatty acid hydroperoxide to the 7,8-dihydroxy derivative was tightly associated, it could not be isolated at that time. It rather took two more years to show that both reactions are catalyzed by the same polypeptide chain (Chao Su et al., 1998). Based on the thus identified sequence (Hörnsten et al., 1999), Tsitsigiannis et al. identified in 2004 a homologue protein in $A$. nidulans that was proposed to be involved in the formation of Psi-factors (Tsitsigiannis, Zarnowski, et al., 2004). Consequently, the

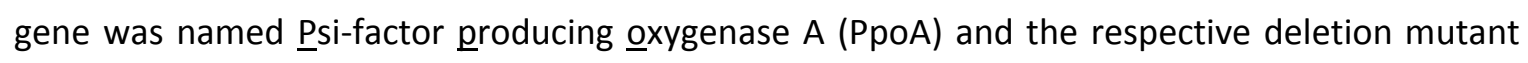
was indeed impaired in spore formation with a higher ratio of asexual to sexual spores in comparison to the wild type. Detailed investigations of the interaction between Ascomycota with a $\Delta p p o$ background and various hosts indicated that psi-factors deploy their action not only in spore formation, but merely are also involved in pathogenicity and colonization of mammal and plant hosts, putatively by regulation of mycotoxin biosynthesis (Tsitsigiannis, Bok, et al., 2005; Tsitsigiannis and Nancy P. Keller, 2006). Expanding this physiological insights into the role of PpoA, LC/MS'-analysis of the oxylipin pattern of $\triangle p p o A$ strains proved that this enzyme is indeed responsible for the formation of the fatty acid $\mathrm{C}_{8}$-hydroperoxy adduct and also the 5,8dihydroxy fatty acid was not detectable in the mutant (Garscha et al., 2007). Since also external addition of the 8-hydroperoxy fatty acid did not restore the formation of the 5,8-dihydroxy derivative, this study concluded that PpoA might exhibit a dual function and catalyzes not only fatty acid peroxidation, but also the subsequent rearrangement of this intermediate to the 5,8dihydroxy fatty acid (Garscha et al., 2007). The subsequent work of Brodhun et al. finally established a heterologous expression system and a purification protocol for this enzyme that enabled to produce pure enzyme in large amounts (Brodhun et al., 2009). Thus, it was possible to perform a detailed biochemical investigation for the first time. The results of this study and a 
subsequent EPR-study (Fielding et al., 2011), have unequivocally shown that PpoA is a bifunctional enzyme that utilizes two distinct heme-domains in order to catalyze the sequential oxidation of fatty acids. The peroxidation of an unsaturated $\mathrm{C} 18$ fatty acid at $\mathrm{C}_{8}$ is catalyzed in the $\mathrm{N}$-terminal domain of PpoA by a peroxidase/dioxygenase and the thus formed intermediate is rearranged to the corresponding 5,8-dihydroxy derivative by a cytochrome P450 activity in the C-terminal domain of PpoA. Hence, this enzyme is not only of interest because of its involvement in regulating fungal growth and pathogenicity, but also a comprehensive system in order to study the versatility of heme chemistry in nature. The two subsequent sections are dedicated to give a synopsis over these two fields of interest and highlight the role that Ppo-enzymes play in these respects.

\subsection{Involvement of oxylipins in host-pathogen interaction}

Lipids represent a heterogeneous class of biomolecules that are defined solely by their solubility in organic solvents. Besides their commonly known function in energy storage and cell compartmentalization, certain lipids (e.g. diacylglycerol) can deploy an important role in signaling (Stryer, 2007). Within this field oxylipins, i.e. oxidized fatty acids, occupy an important position in mammals and plants and their regulatory role in stress response and developmental processes is thoroughly investigated (Funk, 2001; Wasternack, 2007). Exemplary for oxylipin's hormone-like action in defense-responses, jasmonate and its derivatives were shown to be induced upon wounding in tomato and thale cress (Wasternack, 2007) and eicosanoids are widely known for their role in regulating pain, fever and inflammation (Funk, 2001), a fact exploited by all non-steroidal anti-inflammatory drugs (NSAIDs) on the market. Despite these information on the situation in plants and mammals, the knowledge about oxylipins in fungi started to rise rather recently (Andreou et al., 2009; Brodhun and Feussner, 2011). Not only the finding that several fungal enzymes seem to combine two distinct steps of oxylipin-biosynthesis, namely lipid peroxidation and subsequent peroxide rearrangement, in one polypeptide chain (see Section 1.4) renders this a research field of interest, but eventually more fascinating is the idea that the fungal oxylipins might mimic and interfere with host derived oxylipins in order to overcome an established defense mechanism (Tsitsigiannis and Nancy P. Keller, 2006; Christensen and M.V. Kolomiets, 2011). Before reviewing the experimental evidences for this hypothesis (section 1.2.2), the impact of fungal pathogens on agriculture as well as the consumer's health will be outlined briefly. 


\subsubsection{FUNGAL INFECTIONS AND MYCOTOXIN PRODUCTION-A CURSE FOR AGRICULTURE}

Molding of spices, fruits and crops is not just of academic interest but actually causes severe yield depressions. Contamination of the remaining harvest with mycotoxins and the resulting thread of the consumer's health make it even more important to understand the processes of fungal pathogenicity and to establish efficient strategies of plant protection. Mycotoxins are toxic secondary metabolites, produced by fungi in order to promote the fungal infection. Most important are those toxic to humans. Based on a consideration of natural abundance, toxicity and possibility to serve as an indicator analyte, the European Union has regularized the maximal tolerated concentration of six different mycotoxins in food ${ }^{1}$. Thus, those six compounds can be identified as main mycotoxins with severe relevance regarding economic and health issues and their occurrence and toxic effects will be briefly reviewed here (Coppock and Jacobsen; Frisvad et al., 2006):

\section{Aflatoxins (Figure1)}

Aflatoxins can be divided into Aflatoxin B1, B2, G1, G2 and M1. Aflatoxin B1 is the most potent known natural carcinogen. Even if only trace amounts of these compounds are ingested, acute liver toxicity can be observed. Aflatoxins are produced by various Aspergillus species, e.g. A. flavus and $A$. parasiticus. Since those fungi grow under rather tropical conditions, the possibly contaminated foods comprise of nuts, figs, spices etc. But also inadequate storage conditions, i.e. high temperature and humidity, can lead to a severe contamination. An exceptional position within the aflatoxin family is attributed to Aflatoxin M1. This compound is the product of a biotransformation and is yielded by hydroxylation of Aflatoxin B1, if contaminated food is ingested by a cow. Consequently, this toxin can be identified in milk and products thereof.

\section{Fumonisins (Figure1)}

As Tricothecenes and Zearalenone, Fumonisines are produced by Fusarium species and thus can contaminate crops on the field even in a zone of moderate climate. Fumonisines are mainly detected on maize and their major producers are $F$. verticillioides and $F$. proliferatum.

\section{Ochratoxin A (Figure1)}

Ochratoxin $A$ is a nephrotoxic, cancerogenic and teratogenic metabolite, produced mainly by $A$. ochraceus, A. westerdijkiae and some strains of $A$. niger. As for the Aflatoxin producing fungi, those mainly contaminate food grown in tropical zones, e.g. grapes, coffee and cacao beans. In contrast, Penicillium verrucosum is the main contaminant, producing Ochratoxin A in stored food.

\footnotetext{
${ }^{1}$ current regulations regarding maximal levels can be found in: COMMISSION REGULATION (EC) No 2174/2003 for Aflatoxines; COMMISSION REGULATION (EC) No 123/2005 for Ochratoxin A; COMMISSION REGULATION (EC) No 455/2004 for Patulin; COMMISSION REGULATION (EC) No 856/2005 for Deoxynivalenol, Zearalenon and Fumonisine
} 
Introduction

\section{Patulin (Figure1)}

Patulin is a metabolite with toxic effects on prokaryotes as well as eukaryotes. It can be identified in apples, but also in pears and grapes. The major source is Penicillium expansum.

\section{Tricothecenes (Figure1)}

Tricothecenes are a diverse group of compounds with a common lead structure. Of those derivatives Deoxynivalenol is the one that can be regarded as indicator compound and therefore its level in food is regularized. This mycotoxin, which's trivial name vomitoxin illustrates its major toxic effect, is mainly produced by F. graminearum and F. culmorum. Besides Deoxynivalenol, $\mathrm{T} 2$-and $\mathrm{HT} 2$-toxin, which are produced by F. sporotrichioides and F. langsethiae and occur mainly in oat, are discussed to be regularized by law, since those derivatives are known to be highly toxic.

\section{Zearalenone (Figure1)}

Zearalenone has a structure, comparable to the one of the hormone estrogen and its toxic effects mainly derive from this structural similarity. The pseudo estrogen interferes with various processes, regulated by estrogen. The main producers of this compound are $F$. graminearum and F. culmorum.

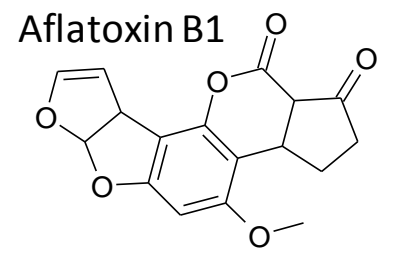

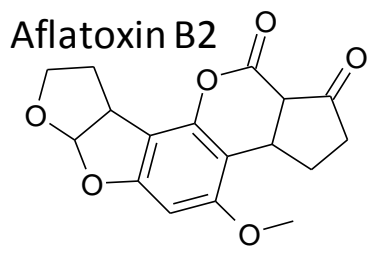<smiles>COc1cc2c(c3oc(=O)c4c(c13)CCCC4=O)C1CCOC1O2</smiles>

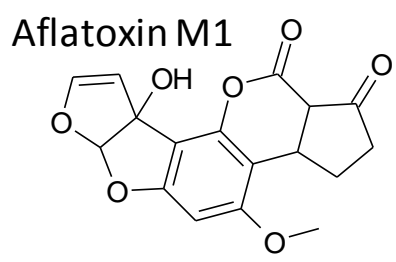

Tricothecene

Patulin

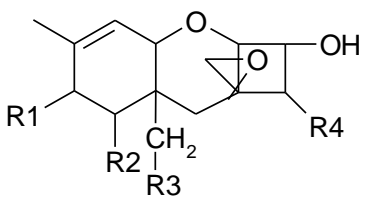<smiles>O=C1C=C2C(=CCOC2O)O1</smiles>

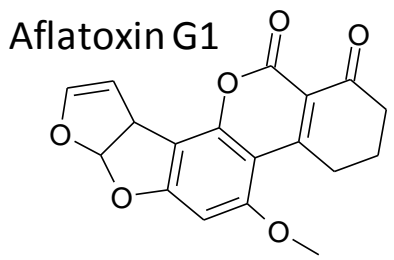

\section{Ochratoxin A}<smiles>CC1Cc2c(Cl)cc(C(=O)NC(Cc3ccccc3)C(=O)O)c(O)c2C(=O)O1</smiles>

Zearalenone<smiles>C=C(OC(C)CCCCC(=O)CCCC)c1c(O)cc(O)cc1/C=C\C</smiles>

Deoxynivalenol: $\mathrm{R} 1=\mathrm{O} ; \mathrm{R} 2=\mathrm{OH} ; \mathrm{R} 3=\mathrm{OH} ; \mathrm{R} 4=\mathrm{H}$ T2-Toxin: $\mathrm{R} 1=\mathrm{OCOCH} \mathrm{CH}_{2}\left(\mathrm{CH}_{3}\right)_{2} ; \mathrm{R} 2=\mathrm{H}$; $\mathrm{R} 3=\mathrm{OCOCH}_{3} ; \mathrm{R} 4=\mathrm{OCOCH}_{3}$ HT2-Toxin: $\mathrm{R} 1=\mathrm{OCOCH}_{2} \mathrm{CH}\left(\mathrm{CH}_{3}\right)_{2} ; \mathrm{R} 2=\mathrm{H}$; $\mathrm{R} 3=\mathrm{OCOCH}_{3} ; \mathrm{R} 4=\mathrm{OH}$

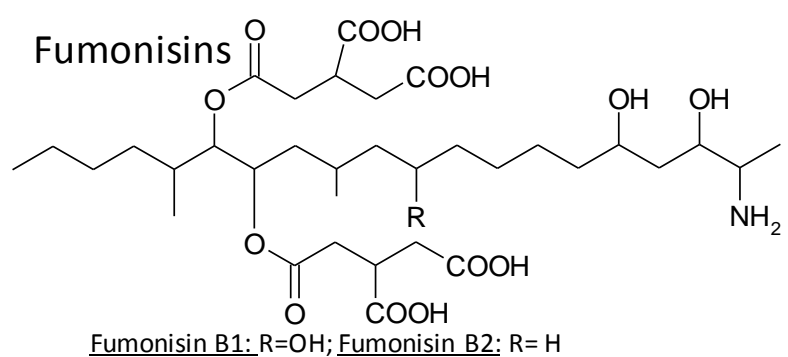

Figure 1: Structure of economical important mycotoxins 
All molds known to be important producers of mycotoxins, e.g. those belonging to the genera Aspergillus, Fusarium and Penicillium, can be classified in the phylum Ascomycota. One of the best established model organisms of this phylum is Aspergillus nidulans. Although not synthesizing one of the regularized mycotoxins, this fungus produces Sterigmatocystin a polyketide related to Aflatoxin and exhibiting similar toxological effects (D.W. Brown et al., 1996). Since the established tools and knowledge might help to gain new insights in the mechanisms and molecules regulating mycotoxin production and pathogenicity of ascomycetes in general, $A$. nidulans can also serve as model organism in this respect.

\subsubsection{THE IMPACT OF OXYLIPINS IN GENERAL AND PSI-FACTORS IN SPECIAL ON FUNGAL PATHOGENICITY}

One of the substance classes that are involved in regulation of crop molding are lipids. In the early 1980's it was shown that aflatoxins are preferentially found on seeds containing high amounts of unsaturated fatty acids and a subsequent study could further narrow down the responsible compounds and establish that oxidized fatty acids are accountable for this enhanced mycotoxin biosynthesis (A. A. Fabbri et al., 1983; C Fanelli and A A Fabbri, 1989). Moreover, a study investigating the role of polyunsaturated fatty acids as well as their hydroperoxy fatty acid derivatives on development of Aspergillus species established their growth and sporogenesis promoting function (Calvo et al., 1999). In line with this observation, Burow et al. showed that mycotoxin synthesis is inversely regulated by two positional isomers of hydroperoxy linoleic acid (HPODE): While 9-HPODE was shown to specifically enhance mycotoxin biosynthesis, 13-HPODE had the antagonistic effect and suppressed mycotoxin production (Burow et al., 1997). Consequently, maize with a mutation in the 9-HPODE synthesizing lipoxygenase (LOX) was less susceptible to Fusarium verticillioides and showed a significantly reduced fumonisin B1 level (Gao et al., 2007). Interestingly, the same maize knock-out line was more susceptible to Aspergillus flavus and displayed increased aflatoxin levels (Gao et al., 2009). This observation together with a study investigating the effect of fungal 13-HPODE on Ochratoxin A biosynthesis (Reverberi et al., 2010) leads to the hypothesis that fatty acid hydroperoxides may generally cause an unspecific up-regulation of mycotoxin biosynthesis, while some plant-derived oxylipins might act as defense mediators and inhibit this process in a pathogen-specific manner.

The virulence enhancing effect of hydroperoxy fatty acids may be explained by the fact that structurally related oxylipins on the one hand mediate host defense and are on the other hand important regulators of fungal development. Exemplary, it is known that in plants jasmonic acid acts inter alia as signaling molecule during wounding and pathogen attack (Wasternack, 2007). However, the effect of this compound on different Aspergillus species is inconsistent. There are reports of Aflatoxin biosynthesis repression in A. flavus grown on medium supplemented with methyl jasmonate (Goodrich-Tanrikulu et al., 1995) as well as on an Aflatoxin biosynthesis promoting effect for A. parasiticus grown under similar conditions (Vergopoulou et al., 2001). 
Interestingly, it was reported that several fungi are able to synthesize this plant defensemediator or a mimic thereof (Brodhun and Feussner, 2011) and it was proposed that these compounds might promote the fungal infection. Albeit this is not in agreement with the role of jasmonic acid as defense-mediator, the fungus might abuse the diverse physiological roles of this oxylipin in plants and an elevated jasmonic acid concentration may induce a senescence process making the plant more susceptible to fungal infections or a better host for necrotrophic pathogens (Thatcher et al., 2009). For the sake of completeness it should be mentioned that also non-plant hosts like mammals produce oxylipins that can mediate fungal growth. Exemplary it was shown that Prostaglandin $E_{2}$ can affect Aspergillus development (Tsitsigiannis, Bok, et al., 2005). Since the first intermediate in the biosynthesis leading to oxylipins is a peroxidized fatty acid in all potential hosts, it is tempting to assume that these intermediates might be sensed by the pathogen to initiate its virulence and finally overcome the established defense response.

With the description of Psi-factors as fungal hormones in the late 1980's (S P Champe et al., 1987; S P Champe and el-Zayat, 1989; Mazur et al., 1991), fungal oxylipins, which structurally resemble these peroxidized fatty acids, were identified. These compounds have been shown to be involved in the balance and regulation of the sexual and asexual life cycle of the fungus (S $P$ Champe et al., 1987; S P Champe and el-Zayat, 1989). Later on supported by the biochemical analysis of Garscha et al. (Garscha et al., 2007), various studies conducted in the group of Nancy Keller could show that these Psi-factors are produced by an enzyme family, which is specific for ascomycetes and shows homology towards mammalian prostaglandin $\mathrm{H}_{2}$ synthase. Physiologically, the respective knock out mutants were impaired in the regulation of their normal life-cycle (Tsitsigiannis, Kowieski, et al., 2004, 2005; Tsitsigiannis, Zarnowski, et al., 2004), a finding which is consistent with the function of Psi-factors as postulated by Champe and coworkers (S P Champe et al., 1987; S P Champe and el-Zayat, 1989). For instance, an A. nidulans strain in which the ppoA gene was disrupted did not produce the 8- hydroxy derivative of linoleic acid and showed a four-fold increased ratio of asexual to sexual spores as compared to a wild type strain (Tsitsigiannis, Zarnowski, et al., 2004) whereas disruption of the ppoC gene resulted in a decreased ratio of asexual to sexual spores and suppressed the biosynthesis of the 8hydroxy derivative of oleic acid (Tsitsigiannis, Kowieski, et al., 2004). Therefore, the identified genes in the model organism $A$. nidulans have been consequently named Psi-factor producing oxygenases $A$ through $C$ (ppoA, ppoB, ppoC). Benefiting from the recent improvements in genome sequencing (Bornscheuer et al., 2012), it became obvious that Ppo-enzymes with their domain-architecture comprising of an $\mathrm{N}$-terminal dioxygenase and a C-terminal cytochrome P450 may be ubiquitously distributed in ascomycetes (Andreou et al., 2009; Brodhun and Feussner, 2011) and hence might exhibit a pivotal function in this ecological niche. Considering that spore development is an essential process in fungal growth and virulence (Calvo et al., 1999) and keeping in mind that the structures of the signal molecules regulating the respective process are similar to that of the plant hormones defending the host, one may hypothesize that host oxylipins might be able to promote fungal pathogenicity. Supporting this hypothesis, 
various Ppo-knock out mutants are not only impaired in spore formation, but exhibit an additional change in their ability to colonize a host, which suggests a certain role of psi-factors in pathogenicity. This effect may be explained by the hypothesis that Psi-factors regulate not only the life cycle, but also the secondary metabolism of the fungus. Exemplary an A. flavus strain, in which four ppo and one lox gene were down regulated exhibited an impaired regulation of Aflatoxin biosynthesis (S.H. Brown et al., 2009) and Tsitsigiannis et al. could show that the Ppoknock out strains of $A$. nidulans were impaired in the colonization of peanut seeds, which is most likely caused by a reduced amount of Sterigmatocystin produced (Tsitsigiannis and Nancy P. Keller, 2006). This mycotoxin is related to Aflatoxin and therefore a potent virulence factor of host colonization. Additionally, regulation of fungal virulence by Psi-factors may point out the existence of an inter-species communication mediated by oxylipins. This cross-talk between host and pathogen may be characterized by perception of foreign oxylipins enabled through chemical similarities of the signaling compounds in all kingdoms of life (Tsitsigiannis and Nancy P. Keller, 2006; Brodhun and Feussner, 2011; Christensen and M.V. Kolomiets, 2011). Although a number of evidences accumulated during the last decade, which support such an oxylipin mediated crosstalk between host and pathogen, no conclusive details of the underlying mechanisms were given to date and some of the results seem to be in conflict with each other. However, a striking evidence for this crosstalk between host, irrespective whether plant or mammalian host, and pathogen was presented when Brodhagen et al. showed in 2007 that a plant LOX can complement the phenotype of $A$. nidulans $\Delta$ ppo strains (Brodhagen et al., 2007). The functional substitution of fungal oxylipins by host derived ones might explain how the fungus can sense the presence of a host and initiate its virulence mechanisms, e.g. production of mycotoxins. So far the evidences collected seem to point out that this mechanism is of general relevance and that oxylipins play a pivotal role not only for the infection of plants but also in mycosis of mammals. However, some hosts may also take advantage of the conserved chemical structure of oxylipins and utilize the pathogen's oxylipins to initiate an adequate defense mechanism (Tsitsigiannis, Bok, et al., 2005). Taken together the role of lipids and especially oxylipins in fungal pathogenicity seems to be worth a further investigation. However, to finally establish a detailed mechanism of how Psi-factors may mediate fungal virulence, more systematic research is indispensable and many aspects of the proposed oxylipin mediated inter-species communication have to be characterized in the future. For instance it is completely unknown by what receptors fungi perceive oxylipins. 


\subsection{A mechanical view on PpoA, an fascinating enzyme to study heme-chemistry}

In PpoA two independent activities are combined in one enzyme: A dioxygenase (DOX) in the Nterminal part of the enzyme catalyzes dioxygenation of $\mathrm{C}_{8}$ of an unsaturated $\mathrm{C} 18$ fatty acid and an atypical $\mathrm{P} 450$-functionality in the $\mathrm{C}$-terminal domain catalyzes the rearrangement of this $\mathrm{N}$ terminally formed hydroperoxy fatty acid to its 5,8-dihydroxy derivative (Brodhun et al., 2009). Interestingly, both domains require a heme cofactor for their activity and, since a different coordination causes a distinct reactivity, the entire enzyme represents two avenues, how versatile heme chemistry can be utilized by nature. Remarkably, this fusion is both a blessing and a curse. While it enables to investigate typically separated activities and their interplay within one system, this is consequently accompanied by an increased complexity of the system. Not solely the interaction and combination of two activities within one enzyme is of interest, but also the peroxide isomerase domain of the enzyme represents an activity that is atypical for cytochrome P450 enzymes. Most likely the P450-domain can be clustered with class III cytochrome P450s that are defined as cytochrome P450s that circumvent the need of external electron donors (Werck-Reichhart and Feyereisen, 2000; Brash, 2009). This class of cytochrome P450s does not insert molecular oxygen in the substrate molecule, but rather rearranges fatty acid peroxides. Notably, all known members of this class are responsible for the biosynthesis of oxylipins and short-cut the commonly accepted P450 reaction cycle by a hemolytic cleavage of the peroxide's O-O bond (Brash, 2009). In contrast, the reaction finally leading to a dihydroxy fatty acid suggests a heterolytic fission of the peroxide's O-O bond and a shunt that shortcuts the P450 reaction cycle by a different path than the previously mentioned reaction (Kupfer et al., 2001).

The following sections will review the current knowledge about similar enzymes that could serve as a guide to elucidate structural and mechanistic aspects of the respective enzyme domain. One should keep in mind that there are only homologue systems for the single domains, but the combination of both activities in one enzyme is something unique. 


\subsubsection{DioxygenASE-Domain}

Sequence alignments of the DOX-domain indicated that it is a member of the myeloperoxidase family (Daiyasu and Toh, 2000). Besides Ppo-enzymes, two members of this family catalyze the dioxygenation of fatty acids: prostaglandin $\mathrm{H}_{2}$ Synthase (PGHS) and $\alpha$-dioxygenase ( $\alpha$-DOX) (Daiyasu and Toh, 2000). Both employ a nearly identical mechanism of catalysis (Mukherjee et al., 2010; A.-L. Tsai and Kulmacz, 2010). This mechanism involves two activities, a peroxidase and the actual DOX, which are mechanistically linked (Figure 2). Initially, within a redox reaction catalyzed in the enzymes peroxidase site, a peroxide molecule is reduced to the corresponding hydroxide and the enzyme's heme cofactor is oxidized two-fold. This reactive heme species (compound I; $[\mathrm{Fe}(\mathrm{IV})=\mathrm{O} \mathrm{PPIX}]^{+*}$ ) is subsequently reduced by transfer of one electron from a tyrosine, which thus forms a reactive radical. The resulting heme derivative (compound II; $[\mathrm{Fe}(\mathrm{IV})=\mathrm{O} \mathrm{PPIX}]$ ) is still a reactive species and responsible for an enzyme inactivation process that is caused by heme destruction, which is illustrated as bleaching of the soret peak, and can be dampened by addition of reducing equivalents that will reduce the heme back to its ferric state (Wu et al., 2007). While these reactions occur in the enzymes peroxidase site (Figure 2, pale red), the actual DOX reaction is mediated by the mentioned tyrosyl radical that is placed within a second active site of the enzyme (Figure 2, pale green). Since its proposal in 1988 (Dietz et al., 1988; Karthein et al., 1988), growing evidence has accumulated that this "branched-chain" model, which mechanistically links both active sites by the intramolecular electron transfer from the catalytic tyrosine in the DOX-site to the oxidized heme in the peroxidase site, indeed is valid for the reaction of PGHS (A.-L. Tsai and Kulmacz, 2010) and the related $\alpha$-DOX (Gupta et al., 2008a; Mukherjee et al., 2010). Within the once activated DOX-site (Figure 2, pale green), the tyrosyl radical interacts with the fatty acid and abstracts a hydrogen atom from either $C_{13}$ of arachidonic acid (PGHS) or $\mathrm{C}_{2}$ from a range of different fatty acids in $\alpha$-DOX. The resulting substrate radical is delocalized over five carbons in case of arachidonic acid and finally reacts with molecular oxygen at $C_{11}$ to produce a fatty acid peroxyl derivative (Wu et al., 2011). In contrast to PGHS, hydrogen abstraction and oxygen insertion occur suprafacial in $\alpha$-DOX and the carbon from which the hydrogen is initially abstracted is identical with the one finally reacting with molecular oxygen (Mats Hamberg, León, et al., 2002). While in PGHS several rearrangement reactions follow the formation of the first peroxyl radical, sequentially including formation of an endoperoxide between $C_{9}$ and $C_{11}$, formation of a cyclopentane ring and a further dioxygen insertion at $C_{15}$, the final step for both enzymes is again an interaction between the catalytic tyrosine and the afore formed peroxyl radical. A hydrogen atom is transferred from the amino acid to form both: a hydroperoxy fatty acid that is released as enzymatic product and a tyrosyl radical that is capable of catalyzing the next reaction cycle upon formation of a new enzymesubstrate complex. The studies of Brodhun et al. (Brodhun et al., 2009) and Fielding et al. (Fielding et al., 2011) could already establish that the dioxygenation mechanism of PpoA resembles the here presented mechanism. EPR-spectra as well as the absence of dioxygenase 
activity in the respective phenylalanine variant indicated that the catalytic competent tyrosine of PpoA is Tyr374, the position homologue to the conserved tyrosine in myeloperoxidases.

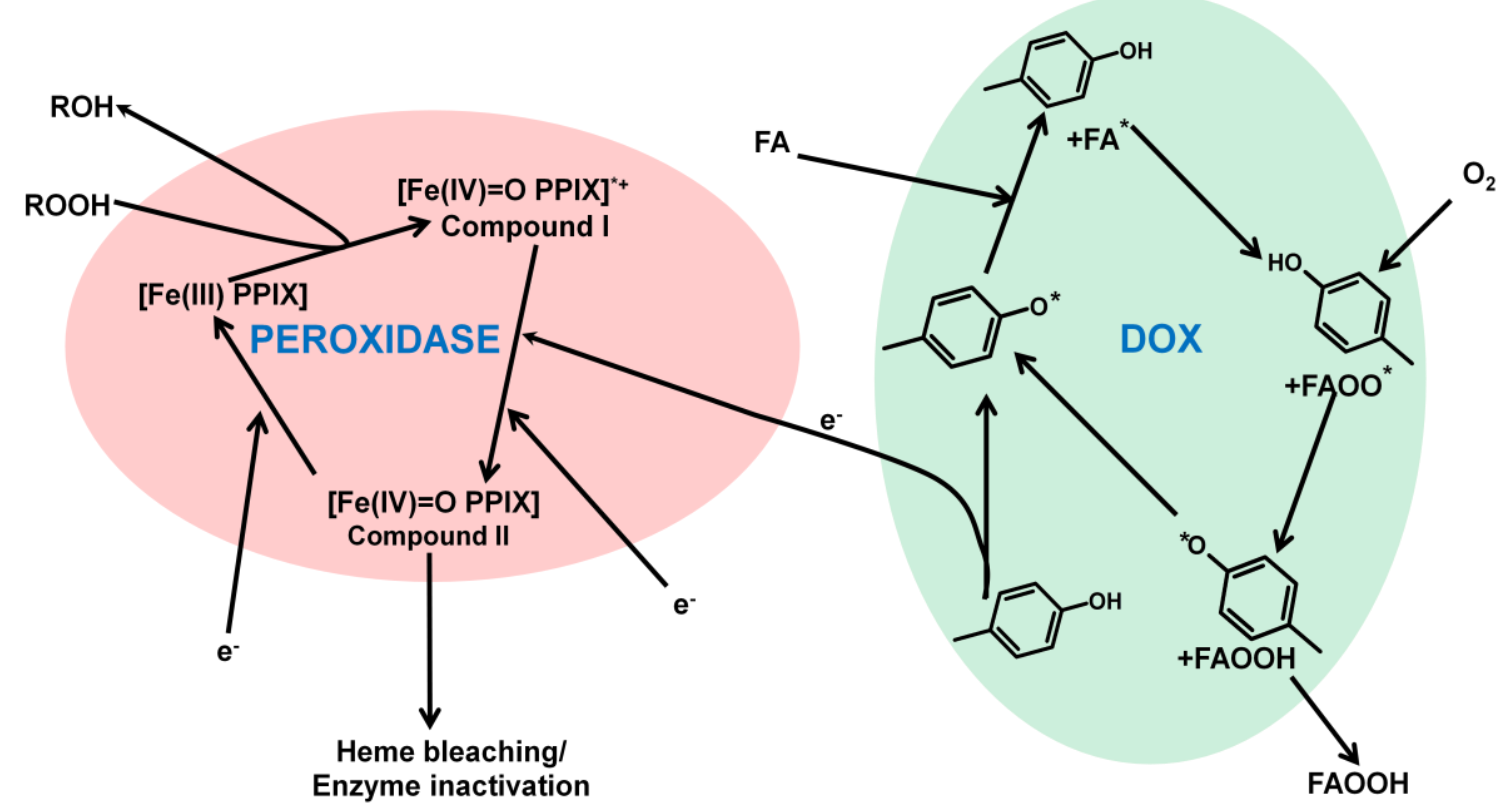

Figure 2: Conserved reaction mechanism of fatty acid oxidizing enzymes, belonging to the myeloperoxidase family. Note that these enzymes have two distinct active sites. In one of these sites dioxygenation of the fatty acid (FA) is mediated by a tyrosyl radical (pale green), while in the mechanistically linked peroxidasesite a peroxide is reduced to oxidize the heme cofactor (Fe containing protoporphyrin IX; [Fe(III)PPIX]), which is reduced again by intramolecular electron transfer from the catalytic tyrosine and thus activates the enzyme (pale red). ROOH denotes a variety of different peroxides and radicals are marked by an asterisk (*). A detailed description of the catalytic cycle is given in the text.

In contrast to knowledge on the mechanism, structural data is only available for PGHS. Although rice a-DOX has been crystallized successfully (Lloyd et al., 2006), so far no solved structure is available. Nevertheless two experimentally verified homology models suggest that the structure resembles the one of PGHS (W. Liu et al., 2004; Koszelak-Rosenblum et al., 2008) pointing out the structural conservation within the myeloperoxidase family. As expected by sequence homologies, the 3D-structures of both PGHS isoforms have a conserved fold. These two PGHSisoforms catalyze the same reaction, but differ in their substrate promiscuity, which might be explained by some specific amino acid exchanges within the DOX active site (Vecchio et al., 2012). However, the main difference between both isoforms is that PGHS-1 is constitutively expressed, while PGHS-2 is the inducible isoform exhibiting a pivotal role during infections and inflammation processes (D.L. Simmons et al., 2004). Both isoforms can serve as prototypes for monotopic membrane proteins (Fowler and Coveney, 2006). They form homodimers and both monomers exhibit a hydrophobic membrane binding domain with which the protein is anchored to the membrane (Michael Garavito et al., 2002). While the peroxidase-site of the enzyme is in proximity of the heme, the entrance to the channel forming the DOX active-site is located at the interface of the membrane binding domain (Figure $3 \mathrm{~A}$, green) and the catalytic domain (Figure 3 
A, blue) and the fatty acid enters the DOX channel from the membrane. At the junction of membrane binding domain and catalytic domain an arginine interacts with the negatively charged carboxyl group of the fatty acid substrate (Figure 3 B). Although structurally conserved, the contribution of this arginine to substrate binding differs remarkably between the two PGHS isoforms: While in PGHS-1 apparently an ionic interaction confers strong substrate binding, in PGHS-2 a hydrogen bond exhibits much weaker binding strength and contributes less strongly to binding (Rieke et al., 1999). From this observation it became obvious that in PGHS-2 hydrophobic interactions between apolar residues lining the active site and the fatty acid are relatively more important for substrate binding than in PGHS-1. In both isoforms, the fatty acid substrate is placed in an L-shaped confirmation within the active site of the catalytic domain and the substrate's $C_{13}$ is positioned next to the catalytic tyrosine residue (Figure $3 \mathrm{~B}$ ).
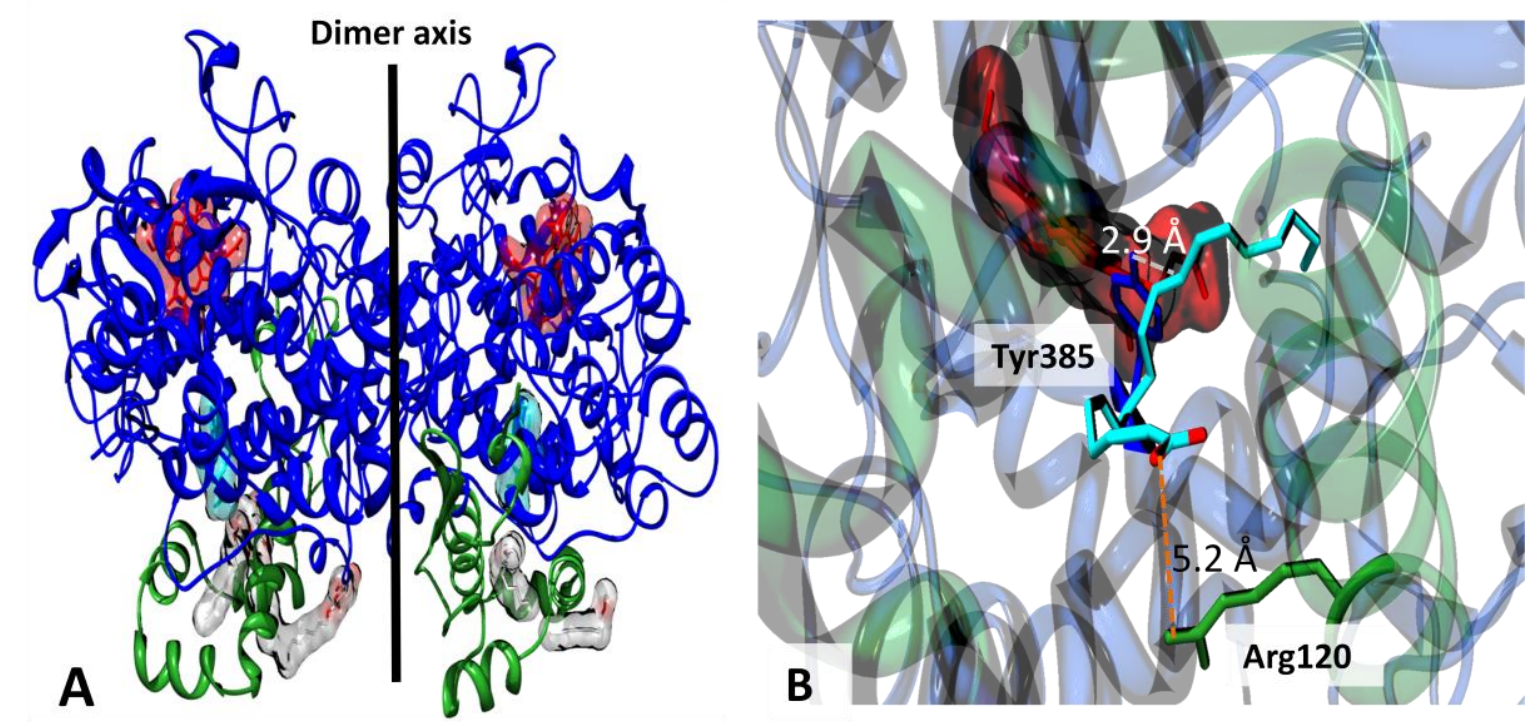

Figure 3: Structure of PGHS. A overall structure of the PGHS-1 homodimer (PDB-ID: 1CQE); heme: red; catalytic domain: blue; membrane binding domain: green; flurbiprofen bound to the active site: cyan; Note the $\beta$-octylglucoside molecules (grey) bound to the membrane binding domain $\mathbf{B}$ Zoom from the membrane into the active site of PGHS-2 (PDB-ID: 3HS5); heme: red; catalytic domain: blue; membrane binding domain: green; arachidonic acid bound to the active site: cyan. Note the two highlighted residues and their interactions with the substrate: Arg120 (5.2 ^ to arachidonic acid's carboxyl group) and Tyr385 (2.9 ^ to arachidonic acid's $C_{13}$ ). 


\subsubsection{Cytochrome P450-Domain}

Cytochrome P450 enzymes are one of the largest enzyme families with a very diverse range of utilized substrates and reactions catalyzed (Werck-Reichhart and Feyereisen, 2000). This diversity is reflected on the level of the primary sequence, on which sequence identity may be as low as $20 \%$ (Sirim et al., 2010). Actually only three residues are completely invariant in all known P450 sequences: the cysteine that serves as the heme iron's fifth ligand and a glutamate/arginine pair that forms the so called ExxR-motif (Werck-Reichhart and Feyereisen, 2000). Despite this low sequence identity, the three dimensional fold of the enzyme core is highly conserved with $12 \alpha$-helices named from A through L, several, short $\beta$-sheets and one conserved coil named 'meander loop' (Peterson and Graham, 1998; Sirim et al., 2010) (Figure 4 A). Noteworthy are the helices I and K (Figure 4 B). The I-helix spans over the distal heme plane and exhibits a conserved threonine which forms a hydrogen bond network with a neighboring glycine. This network causes a kink within the helical structure and thus forms a cavity that most likely accommodates the oxygen to be inserted into the substrate (Martinis et al., 1989). Helix K contains the ExxR-motif, which represents two of the three absolutely conserved residues of P450 enzymes. This motif is placed on the proximal heme site and might be involved in stabilizing the core structure. Besides this core region, the structure of P450 enzymes is nonconserved and variable. This variability enables interaction with different redox partners, optional membrane binding and conversion of a highly diversified substrate spectrum. The substrate promiscuity of P450s and the differences of single enzymes in this respect were additionally described by definition of six so called substrate recognition sites (SRS) (Gotoh, 1992). While SRS 1 is located in a variable loop connecting the conserved core structure of the enzyme-family, SRS 2, 3 and 4 are located within the conserved $\alpha$-helices and SRS 5 and 6 are located within the conserved ß-sheets (Figure 4 A) (Sirim et al., 2010).

As mentioned earlier, the hydroperoxide isomerase activity of PpoA can be identified by sequence homology and functional aspects as cytochrome P450 enzyme (Brodhun et al., 2009). Due to the fact that it does not require molecular oxygen or external electron donors, it can be classified to be part of the cytochrome P450 class III enzyme family (Werck-Reichhart and Feyereisen, 2000). Within this class, enzymes rearranging fatty acid peroxides to produce signal molecules in mammals (Cyp8a, prostacyclin synthase and Cyp5, thromboxane synthase) and plants (Cyp74-family, i.e. allene oxide synthase, divinyl ether synthase and hydroperoxide lyase) are systematized (Brash, 2009). Of this class four crystal structures are available and they all exhibit the conserved P450-fold. Notably, the plant members of this P450 class exhibit a specific alteration of the primary sequence. Within the typically highly conserved heme binding loop a nine amino acid insert can be identified (D.-S. Lee et al., 2008). This longer heme binding loop leads also to a slight perturbation of the conserved P450-fold with a change in the mode of heme-binding and a putative disruption of redox partner-interaction (D.-S. Lee et al., 2008; L. Li et al., 2008). 
Introduction
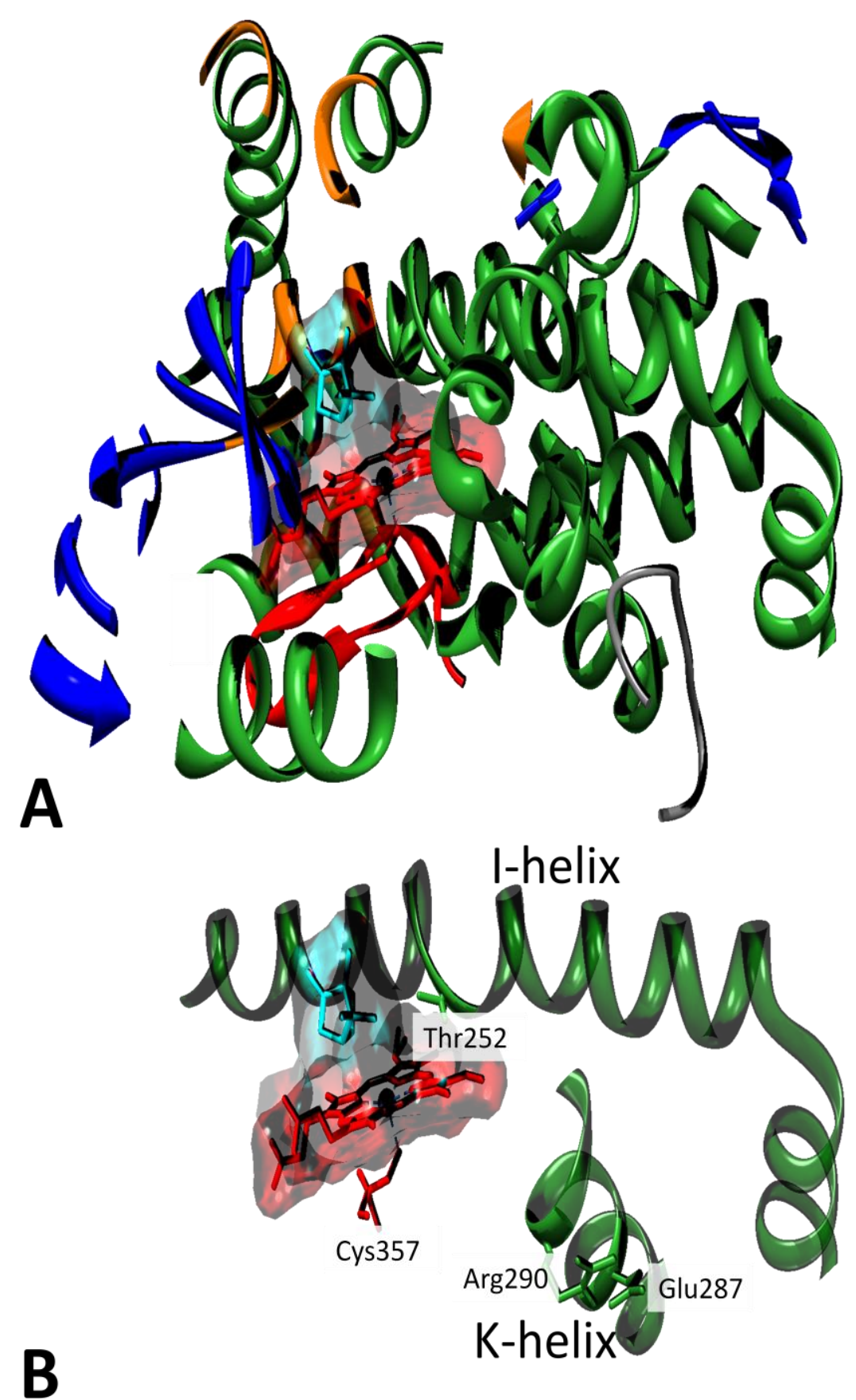

Figure 4:

Conserved

overall-fold of

cytochrome $P 450$

enzymes. The

tertiary structure

of P450 enzymes

is defined by the

presence of 12

conserved $\alpha$ -

helices and

several short $\beta$ -

sheets, while the

variable loops

connecting these

conserved

elements

account for the

diversity of this

enzyme-family.

Exemplary

shown are

secondary

structural

elements

forming the core structure of the prototype enzyme P450cam (PDB: 2ZWT; assigned

according to the algorithm of Sirim et al. (Sirim et al., 2010)). A Conserved helices are depicted in green, while $\beta$-sheets are rendered in blue and substrate-recognition sites are orange. Heme and Heme-binding loop are shown in red and the meander-loop is gray. The substrate (camphor) is rendered in cyan. B Highlighted are the I-helix, which is involved in determination of substrate specificity and harbors a threonine (Thr252 in P450cam) that is proposed to facilitate oxygen activation, and the K-helix, which contains the ExxR-motif that includes two of the absolutely invariant amino acids of P450s (i.e. Glu287 and Arg290 in P450cam; the third invariant amino acid is a cysteine coordinating the heme [Cys357 in P450cam]). The K-helix is proposed to stabilize the heme co-factor. 
The mechanism of catalysis differs remarkably between typical cytochrome P450monooxygenases and class III enzymes, i.e. hydroperoxide rearranging cytochrome P450s (Figure 5). To highlight these differences, a brief outline of the typical P450 reaction cycle is necessary. A more detailed view on this issue is for example given in a review from Denisov et al. (Denisov et al., 2005). In a common P450 reaction the hydroxylation or epoxidation of a substrate is catalyzed and the oxygen needed therefore is derived from molecular oxygen. Preceding the actual reaction, the enzyme has to be activated and the second atom of the molecular oxygen is reduced to water. Reduction equivalents therefore are provided by $\mathrm{NADPH} / \mathrm{H}^{+}$and are delivered by either P450 reductase or the flavoprotein-adrenodoxin system. Consequently, the first half of the typical reaction cycle describes only this enzyme activation by oxygen and no modification of the substrate occurs (Figure 5). The first step of the reaction cycle is replacement of hemebound water by a substrate molecule $\mathrm{RH}$ and a concomitant change of low-spin ferric heme to high-spin ferric heme (Conner et al., 2011). Following a reduction of ferric heme to its ferrous form, dioxygen is bound to the cofactor. A second electron transfer generates compound 0 , an iron-peroxo-heme-intermediate. Although some studies discuss that this species already could be responsible for enzyme reactivity, most calculations and experiments contradict this (P.K. Sharma et al., 2003; A. Franke et al., 2008). Finally a hydrogen atom is transferred to compound 0 which leads to a cleavage of the peroxo-O-O-bond and generation of compound I, the same two-fold oxidized heme species that also can be found as intermediate in enzymes of the myeloperoxidase-family (see section 1.3.1). For this oxygen-bond cleavage, a threonine within the I-helix has been shown to be crucial (Imai et al., 1989; Martinis et al., 1989). The last steps of this typical reaction cycle involve interaction with the substrate. Firstly, heme compound I serves as oxidizing species within a redox reaction, yielding a substrate radical and heme compound II. This heme compound is one-fold oxidized and although in the common representation its ironstructure is described as $\mathrm{Fe}(\mathrm{IV})=\mathrm{O}$, some studies suggest that it is rather $\mathrm{Fe}(\mathrm{IV})-\mathrm{OH}$ (Green et al., 2004). Within the next step of the reaction cycle, this oxygen is rebound to the substrate-radical. This results in an enzyme-product complex where the heme is already in its ferric form. The very last step to complete the reaction cycle is then dissociation of the product from the enzyme.

For later discussion mentioning of two artificial shortcuts of this reaction cycle is necessary. By the first shortcut compound I can be directly yielded without the need of molecular oxygen or reduction equivalents. If the enzyme is treated with peroxides (e.g. hydrogen peroxide or $m$ chloroperbenzoic acid), a redox reaction between heme and this peroxide yields directly the corresponding hydroxide and compound $\mathrm{I}$. The thus charged enzyme can oxidize the substrate in exactly the same manner as if it would have been activated by reduction of molecular oxygen. Surely, the most impressive application of this peroxide shunt was trapping of the highly reactive compound I by Rittle and Green (Rittle and Green, 2010). Recently, two enzymes have been identified that seem to utilize this peroxide shunt for a native reaction: the $\alpha$, $\beta$-hydroxylation of a fatty acid substrate with hydrogen peroxide as oxygen source (Matsunaga et al., 2002; D.-S. Lee et al., 2003; Fujishiro et al., 2011). As presented by Brash, all plant class III cytochrome P450s 
shortcut the outlined reaction cycle by homolytic cleavage of the substrate peroxide's O-O-bond and direct formation of heme compound II (Brash, 2009). Hence, this subclass of cytochrome P450s utilizes fatty acid peroxides as substrate and for enzyme activation. In contrast to the peroxide shunt, the peroxide's O-O-bond is cleaved not heterolytic and therefore compound II instead of compound $\mathrm{I}$ is formed. Thus, the substrate is not fully reduced to the corresponding hydroxide, but an alkoxyl radical is formed. This alkoxyl radical will readily react with the adjacent double bond and form an epoxy allylic carbon radical. Subsequently, this radical looses a hydrogen atom, which in toto classifies this kind of reaction as dehydration. The hydrogen loss itself can be subdivided into two steps: i) transfer of an electron to the heme iron and ii) loss of a proton. The first of these steps restores the cofactor, i.e. it yields ferric heme and a hydroxide, and forms a substrate cation. The subsequent proton loss would form a new double bond and finally furnish either an allene oxide or a divinyl ether. The reaction catalyzed by hydroperoxide lyase and eventually leading to an unstable hemiacetal, which spontaneously decomposes to an aldehyde and an $\omega$-oxo fatty acid, most likely differs from this scheme with no proton loss occurring, but rather a conventional oxygen rebound from compound II. Of course, formation of an epoxy alcohol also involves a classical oxygen rebound to the epoxy allylic radical. For mammalian class III-P450s the same shortcut of the reaction cycle with homolytic peroxide's OO-bond cleavage and compound II as solely heme intermediate was shown to be true (Hecker and Ullrich, 1989; Yeh et al., 2005).

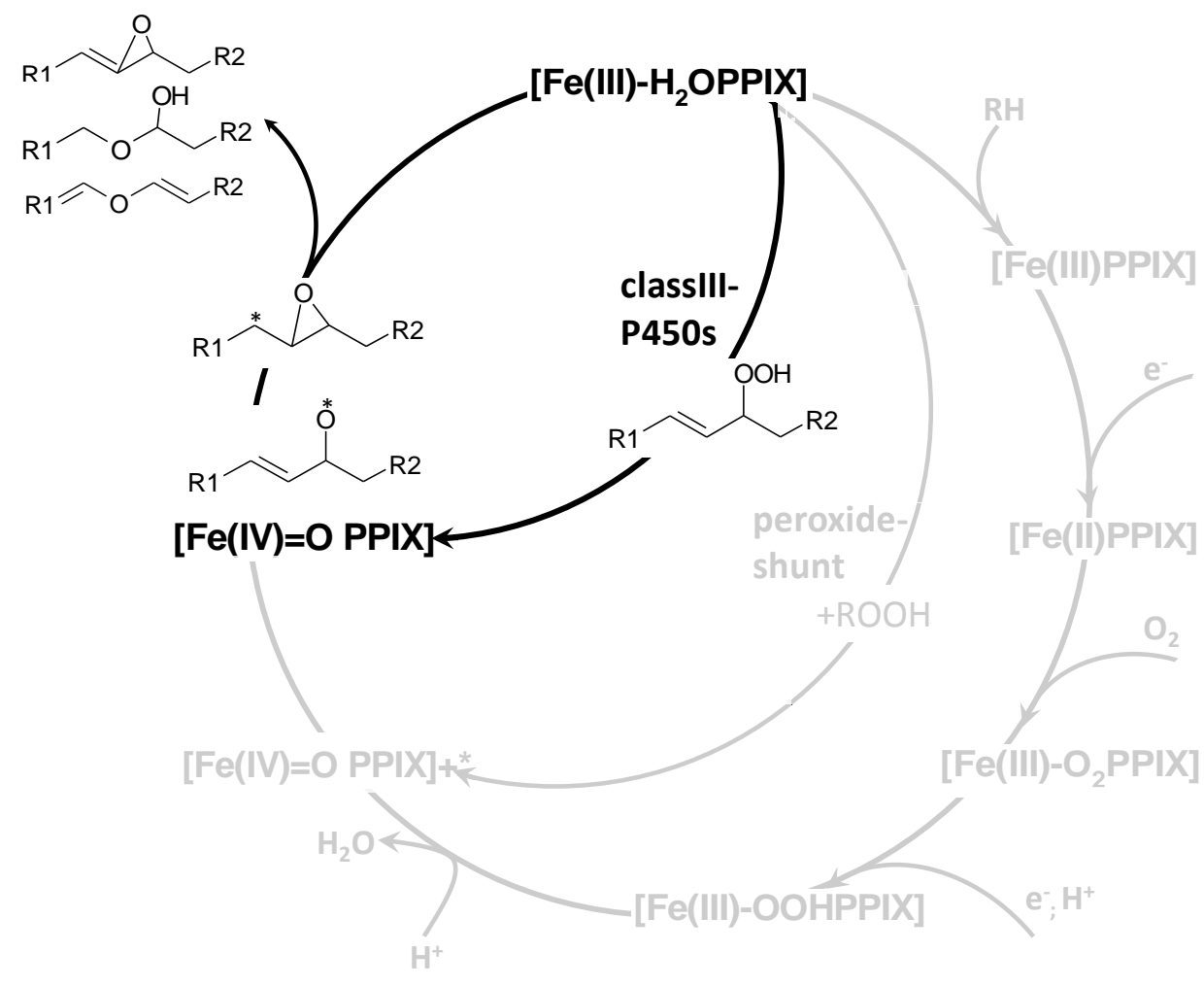

Figure 5: The cytochromeP450 reaction cycle and its shortcut, occurring in catalysis of classIIIP450s. For all known members of this fatty acid peroxide rearranging P450s, it was shown that the full reaction cycle

(grey) is shortcut and that heme compound II is the only reaction intermediate. For illustration reactants and products of Cyp74 enzymes are shown in this figure. 


\subsection{Interaction of DOX-and P450-enzymes in oxylipin biosynthesis}

So far, a hypothesis about the physiological role of oxylipins in host defense was presented, first indications for the use of similar compounds by pathogenic fungi were outlined and enzymes, homologue to the distinct PpoA-domains, were introduced. Interestingly, all of these homologue enzymes are involved in oxylipin biosynthesis in mammals and plants, but so far only single enzymes of complex biosynthetic routes were described. In this section the interplay of these enzymes to compose the entire biosynthetic pathway and finally yield the bioactive compound is summarized. This description will focus on selected examples of biosynthetic routes and one should be aware that there are alternative routes for production of distinct oxylipins (Andreou et al., 2009; Brodhun and Feussner, 2011). Namely the discussion will include the mammalian prostanoid pathway and the LOX-pathway from plants. Roughly spoken both pathways consist of a two-step process. In a first step a fatty acid is functionalized by insertion of molecular oxygen. The thus formed lipid peroxide is further processed by the action of diverse unusual cytochrome P450 enzymes leading to peroxide isomerization and finally formation of the active compound or a precursor thereof. The enzymes involved in oxygen insertion and formation of the hydroperoxy fatty acid can be classified in two big groups. Both groups utilize radical chemistry to perform their reactivity, but differ in their cofactor requirements and their demands on substrate unsaturation (C. Schneider et al., 2007). While lipoxygenases (LOXs) require an iron-cofactor and initiate their reaction on a bisallylic carbon, PGHS and $\alpha$-DOX have a heme cofactor and at least $\alpha$-DOX can catalyze activation of carbons with higher bond dissociation enthalpy. Although both classes are present in plants and mammals, one of the most important oxylipin biosynthesis pathways in mammals is initiated by PGHS, while the prominent oxylipin biosynthesis pathways in plants are initiated by LOX-enzymes (Brodhun and Feussner, 2011). As mentioned previously, important active compounds are formed from the yielded hydroperoxy fatty acid by peroxide isomerizing cytochrome P450 enzymes. In mammals those are thromboxane synthase for synthesis of thromboxane, which is involved in platelet aggregation and vasoconstriction, and prostacyclin synthase, which's product acts as a thromboxane antagonist (Funk, 2001). In plants these unusual cytochrome P450s form an own subfamily: Cyp74. From these Cyp74-enzymes allene oxide synthase (AOS), the first enzyme in the biosynthesis-pathway of jasmonic acid, is probably the most important one (Brash, 2009; Brodhun and Feussner, 2011). This enzyme catalyzes the formation of an allene oxide by dehydration of the fatty acid peroxide. Other enzymes belonging to this P450-subfamily are hydroperoxide lyase (HPL), epoxy alcohol synthase (EAS) and divinyl ether synthase (DES) (Brash, 2009; Brodhun and Feussner, 2011). Considering the results from Brodhun et al. on the domain structure of PpoA (Brodhun et al., 2009), it becomes evident that also the fungal pathway yielding Psi-factors resembles this two-step process, albeit only one enzyme is involved. While existing as two independent enzymes in plants and mammals, within ascomycetes the dioxygenase and the atypical P450-enzyme involved in furnishing the bioactive oxylipin are fused to one polypeptide chain (Figure 6). 

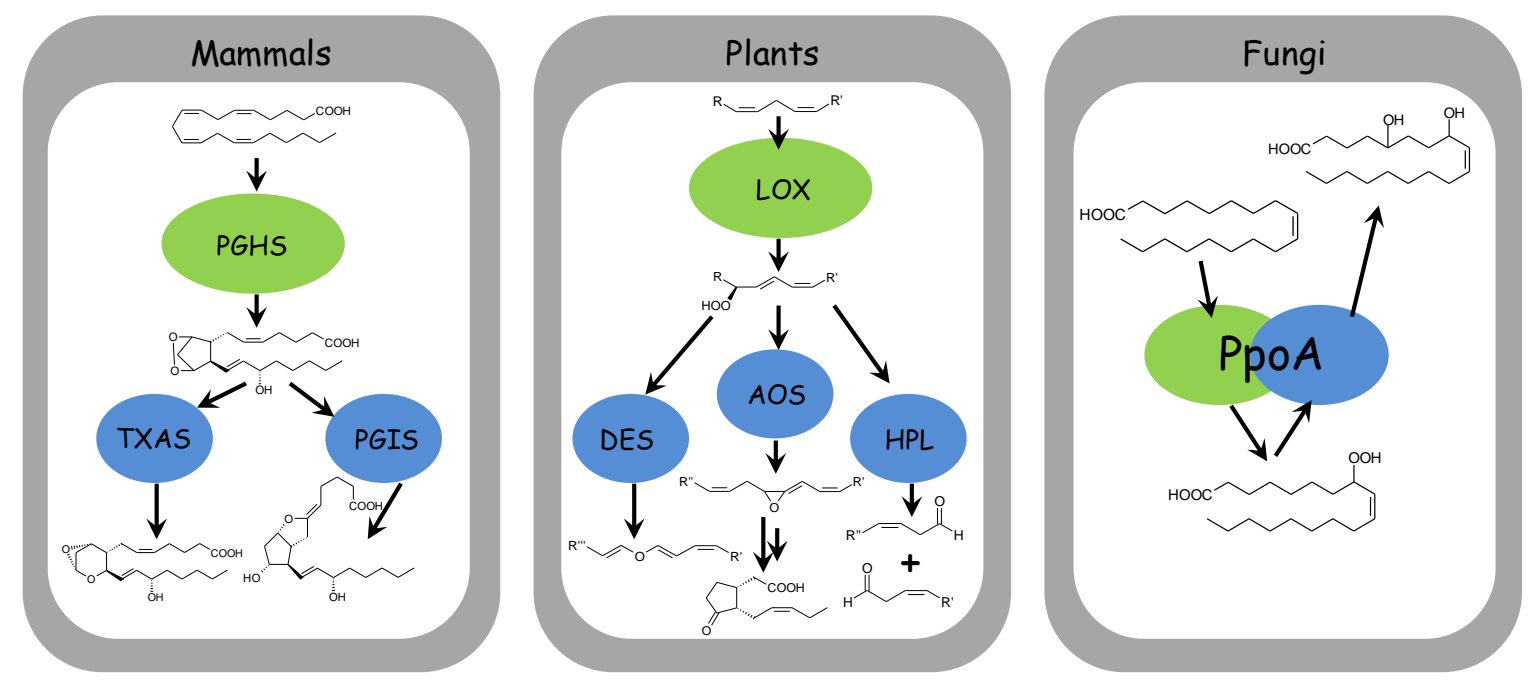

Figure 6: Common two step biosynthesis pathway of oxylipins. In mammals as well as in plants typical pathways of oxylipin biosynthesis involve fatty acid peroxidation by a dioxygenase (green) and subsequent rearrangement of this hydroperoxy fatty acid by an atypical cytochrome P450 (blue). Biosynthesis of fungal Psi-factors was shown to comprise the same reaction steps, but both enzymatic activities are fused to one enzyme. Exemplary substrates, products and intermediates as well as catalyzing enzymes are shown for selected pathways in each kingdom. Abbreviations: PGHS: prostaglandin $\mathrm{H}_{2}$ synthase; TXAS: thromboxane synthase; PGIS: prostacyclin synthase; LOX: lipoxygenase; DES: divinyl ether synthase; AOS: allene oxide synthase; HPL: hydroperoxide lyase; PpoA: Psi-factor producing oxygenase $A$ 
Introduction 


\section{OBJECTIVES AND OUTLINE}

The aim of the present study was to functionally and structurally characterize Psi-factor producing oxygenases and thus elucidate the molecular basis of Psi-factor biosynthesis in ascomycetes. With PpoA as prototype of the Ppo-enzyme family and its expression and purification protocols on hand, the prerequisites for such a study were already established in a previous study (Brodhun et al., 2009). Therefore, the main objective was to derive structural information and link this data with the mechanism proposed in this former study. Considering the size of this enzyme, X-ray crystallography was the method of choice to derive an atomic structure. Despite several efforts to rescue the crystallization project, the prototype Ppo did not yield diffraction quality crystals (Section 4.2). To overcome this dead end, template-based structure prediction was utilized to model the distinct enzyme domains that resemble characterized oxylipin producing enzymes from other species (Section 4.3). Although templatebased structural models tend to be reliable, careful validation of conclusions drawn from these models is mandatory. Therefore, enzyme variants were constructed and thoroughly characterized by various biochemical approaches to finally establish determinants of reactivity and thus link the active site structure to the enzyme function (Section 4.4). Thus the crucial involvement of two tyrosines (Tyr374 and Tyr327) in dioxygenation and an asparagine (Asn887) in hydroperoxy fatty acid rearrangement was shown. Moreover, the modes of substrate binding to the distinct domains were established. While an arginine (Arg336) might ionically bind the carboxylate of the fatty acid substrate in the DOX-domain of PpoA, two phenylalanines (Phe795 and Phe799) seem to be involved in proper substrate placement in the P450-domain. To bridge the gap between the predicted and biochemically validated structures of the single domains on the one hand and their arrangement in a single polypeptide chain as well as the enzyme's quaternary structure on the other hand, small-angle X-ray scattering data of the native enzyme were obtained and the low resolution envelope of the particle was calculated thereof (Section 4.6.1). The results indicate that native PpoA might exhibit a flat trimeric quaternary structure. To additionally shed light on a selected aspect of the enzyme mechanism, specifically dideuterated substrates were used to probe the kinetic contribution of the hydrogen transfer steps occurring during fatty acid conversion (Section 4.7). These measurements revealed that from the three steps involving hydrogen transfer, the tyrosyl radical mediated hydrogen abstraction from $\mathrm{C} 8$ exhibits clearly the most important kinetic contribution. 
Objectives and Outline 


\section{METHODS}

\subsection{Protein crystallization}

For proteins larger than $30 \mathrm{kDa}$ the only possibility to get atomically resolved structural information is to obtain single crystals and subsequent collection of X-ray diffraction data. The main obstacle in this procedure is the identification of conditions under that the protein of interest will crystallize. A comprehensive overview of crystallization techniques is given in literature (Bergfors, 1999). A typical crystallization experiment is set up by mixing a "high" protein concentration with several precipitants to be tested. These precipitants are quite diverse in nature and can be: salts (e.g. $\mathrm{NaCl} ;\left(\mathrm{NH}_{4}\right)_{2} \mathrm{SO}$ ), organic compounds (e.g. polyethylene glycols, methyl pentanediol), organic solvents or various combinations thereof. Initially the precipitant concentration is chosen to be lower than would be necessary to precipitate the protein. This point has to be found for every protein/precipitant-combination empirical. Subsequent, the condition is allowed to slowly increase the concentration of protein and precipitant by controlled evaporation of water. Although several approaches exist to achieve this, the most common used one is vapor diffusion. Here the miniaturized condition with protein and precipitant is enclosed together with a larger reservoir of undiluted precipitant (either as "hanging" or "sitting drop"). The sealing guarantees that water will slowly evaporate from the drop, as long as the precipitant concentration in the drop is lower than in the reservoir. Thus, the drop shrinks and both, the precipitant and the protein concentration are increased. Figure 7 shows and describes an idealized phase diagram for a vapor diffusion experiment. Note that for each examined precipitant, concentrations have to be optimized in order to prevent precipitation and avoid undersaturation. Another typical problem is associated with the fact that nucleation and (optimal) crystal growth are not typically occurring at the same position of the phase diagram. Hence, a too steep increase of concentrations might lead to overnucleation and formation of many small and bad diffracting crystals. Although not usable for the diffraction experiment itself, these tiny crystals still might pave the way to success by serving as starting material in various kinds of seeding experiments. By preventing the need to bring the system to a state where nucleation occurs, these seeding experiments can typically speed up the crystallization experiment and could yield bigger crystals than obtainable without seeds.

Up to date identification of a precipitant condition that gives an initial hit that could be optimized further is still an empirical approach. Nevertheless, automation and miniaturization allows the fast screening of various conditions without too much effort. Typically, screens are available that comprise various conditions (i.e. combinations of different precipitants, additives and buffers), which have been used successfully in crystallization trials of other proteins. These biased, random screens are called sparse matrix screens. Additionally, screens are available that try to rationalize the sampling of the parameter matrix (Grid screens). Caused by the huge 
amount of parameters (e.g. precipitants, additives and buffers with various concentrations and in different combinations), an entirely systematic screen is not feasible and Grid screens deploy their strength mainly in the optimization of an identified initial hit.

Figure 7: Phase diagram of an optimal protein-precipitant condition for vapor diffusion. The axes represent the concentration of precipitant and protein, respectively. The vapor diffusion experiment starts at point 1, where concentrations of protein and precipitant are low enough to ensure protein's solubility. Due to evaporation of water, the drop's volume shrinks and the concentration of protein and precipitant are equally increased. As soon as the nucleation zone is hit (2), crystal nuclei will form and thus deplete the protein concentration, which brings the condition to a phase of slow and

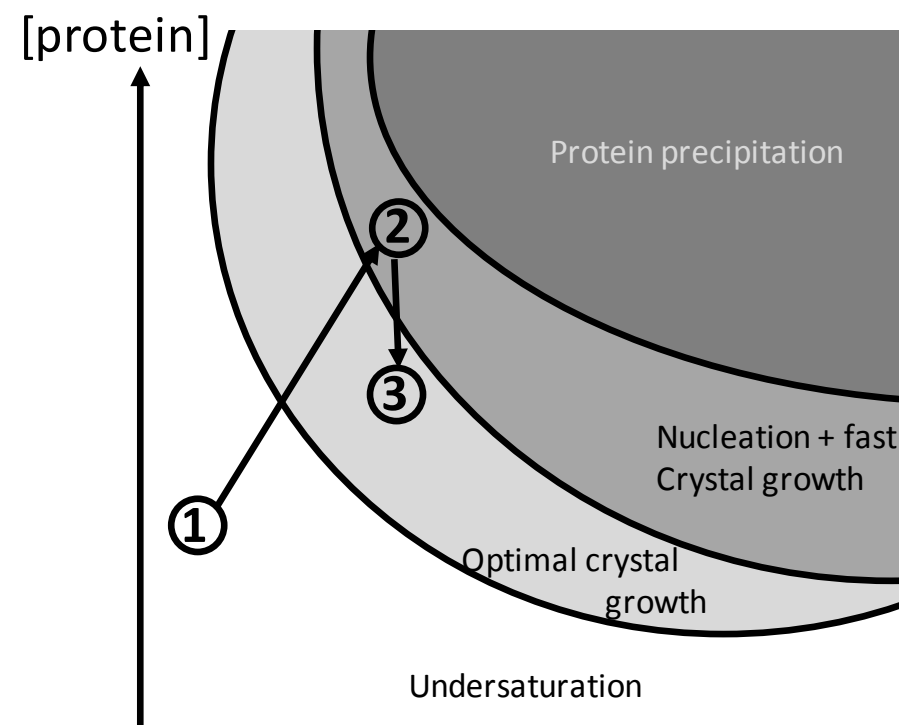

[precipitant] even crystal growth (3).

Despite the technical improvements made to enable automatized high-throughput screening of various conditions, new statistics show that only $10 \%$ of all proteins will crystallize readily (Kim et al., 2008) and that those will most likely show a first promising result even if screening only a small set of different conditions (Z.S. Derewenda, 2004). Thus it would be hardly meaningful to uninspiredly extent the conditions screened, in order to find a suitable condition for a protein that resisted successful crystallization so far. Besides this random trial and error approach with excessive testing of various possible crystallization conditions, one can think of various improvements of the protein in order to yield well diffracting crystals. These include:

-reductive methylation of surface exposed lysines (Kim et al., 2008)

-surface entropy reduction (Z.S. Derewenda and Vekilov, 2006; Cooper et al., 2007)

•construction of fusion proteins/ fixed arm carrier (Smyth et al., 2003; Moon et al., 2010)

-proteolytic digestion/ removal of flexible parts (Wernimont and Edwards, 2009)

-cocrystallization with ligands

-crystallization of homologue (thermostable) proteins

-antibody mediated crystallization (Hunte and Michel, 2002)

-GraFix/ cross linking approach to yield monodisperse complexes for 3D-cryo EM (Kastner et al., 2008)

-a combination of the aforementioned approaches (Moon et al., 2010) 
In the following the advantages and disadvantages of the single techniques will be discussed. In order to form a well ordered crystal lattice the sample has to be homogenous and should consist of molecules with a defined surface that can interact with each other. The first two approaches to optimize the protein, utilized for crystallization, follow the same idea: Charged side chains with long, and therefore intrinsically not well ordered, sidearms are modified in order to facilitate regular contacts between individual protein molecules. Especially for peripheral membrane binding proteins this seems to be of high importance, because these proteins have large, positively charged clusters on their surface, which are supposed to interact with the anionic phospholipids of the membrane (Bhardwaj et al., 2006). While the first approach aims at a biochemical methylation and will only affect surface exposed residues, the second approach consists of site-directed-mutagenesis steps to replace surface exposed glutamic acids and lysines with alanine. This implies all the drawbacks, one always have to keep in mind when dealing with site-directed-mutagenesis. Moreover the identification of surface exposed residues without structure is somewhat empirical and a large surface area might require the mutation of different amino acids, with each and every mutation step having the same inherent threat of structure perturbation or loss of function. While these two approaches modify the protein surface to allow the formation of crystal contacts, the other approaches rather try to yield monodisperse and well structured units for the formation of the crystal lattice. Therefore unordered loops and tails can be removed by limited proteolysis of the native protein. Upon substrate binding enzymes might undergo an induced fit, yielding a higher ordered structure. Another technique that proved to be very valuable for 3D cryo EM is called GraFix. The idea is to obtain monodisperse particles by ultracentrifugation and simultaneous fixation of this state by cross linking with glutaraldehyde. The promise of employing fusion proteins is that the added protein domain will crystallize readily and provide crystal surfaces that might guide the crystallization of the enzyme of interest. Furthermore the structure of the fused protein often is resolved to high resolution and this information can be used to ease the problem of phase determination. Unfortunately, the generated multidomain proteins are often very flexible with respect to the arrangement of the domains. Therefore a rigid linker is needed to assure a homogenous domain architecture, which in turn is a prerequisite for the formation of a defined crystal lattice (Smyth et al., 2003). Fragments of antibodies, raised specifically against the protein of interest can fixate intrinsically disordered, flexible parts of the protein, shield hydrophobic regions and provide new surfaces for crystals. Although nowadays generation of antibodies has become a standard technique, it is still expensive, labor-intensive and time-consuming. If modification of the protein does not lead to well diffracting crystals, a last opportunity is to crystallize a homologue protein. This related protein might be less flexible and therefore more easily to crystallize. 


\subsection{Prediction of protein folds by computer algorithms}

Although a nowadays commonly accepted hypothesis, postulated by the Nobel Prize laureate Christian B. Anfinsen and known as "Anfinsen's dogma", proposes that the protein structure is determined solely by its primary structure (Anfinsen, 1973), prediction of protein folds is still a challenging task. The mentioned dogma, also known as "Thermodynamic Hypothesis", is based on the idea that under physiological conditions all proteins will fold to a state of lowest Gibbs free energy, and therefore the native state can be calculated if all forces governing the interatomic interactions are known. The challenge of protein-folding can be visualized by thinking of the degrees of freedom that exist for the permutation of the atoms of a macromolecule within the three-dimensional space (Zwanzig et al., 1992). While the nature has found a way to reach the native state within physiological reasonable time and thus to overcome this "Levinthal's paradox", sampling of the conformational space in order to identify the global energetic minimum is still the most challenging task in protein-fold prediction. Also state-of-theart computational power allows the de novo fold prediction only for peptides of a limited size, i.e. only a small conformational space is searchable without high risk of getting trapped in a local minimum of the energy function (Yang Zhang, 2008b). Therefore other techniques have been designed that allow to define the initial structure based on homology to a protein with known structure (homology modeling) or by alignment of small sequence strings with known protein folds (fold recognition or protein threading) (Yang Zhang, 2008b). Remarkably, all techniques have gained severe improvements from recent developments. As the free-modeling and ab initio approaches could benefit from improvements in computational power and improved parameterization of their physics-and knowledge-based force fields, the template-based approaches could be utilized more frequently with the advent of more and more experimental structures placed in the protein database (PDB; http://www.rcsb.org/pdb/home/home.do) (Yang Zhang, 2008b). While suitable templates for most protein-domains are nowadays available, modeling of multi-domain proteins is still one of the most challenging tasks. To overcome these problems, one might think of modeling the single domains and subsequent identification of a reasonable spatial assembly by techniques allowing the determination of low resolution protein envelopes (Förster et al., 2008).

There are several algorithms available and in order to assess the reliability of the programs the biyearly competition CASP (Critical Assessment of Techniques for Protein Structure Prediction) is performed. One of the outstanding algorithms within the last three rounds (CASP7-2006; CASP82008; CASP9-2010) was I-TASSER (iterative threading assembly refinement) from the Zhang lab (Yang Zhang, 2008a; Roy et al., 2010). It is not only a highly reliable algorithm, but also allows to predict the structure of large proteins up to 1500 amino acids and hence was considered to be the best choice for modeling of PpoA. Its principle is based on a combination of threading, subsequent assembly and refinement of the model within a proprietary force field (Roy et al., 2010). In a first step, the submitted protein-sequence (and its predicted secondary structure) is 
utilized to identify templates in the PDB database that are best suited to guide the structure building of a given subsequence. As result of this threading process several small folds representing substructures of the final protein model are obtained. In the next step these fragments are assembled to a whole structure. Finally the structure is refined within a force field in order to remove steric clashes and improve hydrogen bonding networks between the assembled fragments.

\subsection{Characterizing the oligomeric assembly of macromolecule by Small-angle X-ray scattering (SAXS)}

Detailed description of SAXS to measure low-resolution particle structures is given in literature (Putnam et al., 2007; Jacques and Trewhella, 2010; Mertens and Svergun, 2010) and here only the essentials of this method are reviewed. The basic principle of X-ray scattering is that charged particles, e.g. electrons, emit electromagnetic waves when they are accelerated. If this acceleration is caused by elastic collision of an electromagnetic wave with a charged particle, this leads to an apparent change in the direction of wave propagation, although rather a secondary wave is generated. Assuming coherent scattering, one can observe intensity patterns of the secondary waves that are caused by constructive or destructive interference. This intensity patterns contain valuable information about the distances between lattice-planes in crystals or the envelope of a molecule in solution, if one considers only the circular scattering intensities at small angles. Hence, SAXS is a technique utilized to determine the envelope of a macromolecular particle. It can be employed in order to measure the oligomeric state of a protein sample, determine the assembly of single domains to an entire molecule or asses the flexibility of a protein which's structure was previously solved by high resolution techniques (i.e. NMR or crystallography) or can be predicted reliably.

During data collection the beam intensity is measured in dependence of momentum transfer $q$

( $\mathrm{q}=4 * \pi * \frac{\sin (\theta)}{\lambda} ; \lambda=$ wavelength and $2 * \theta=$ angle between incident beam and scattered beam). The measured intensity is typically represented in a one dimensional plot as radially averaged mean (Figure $8 \mathrm{~A}$ ) and depends on the particle's shape and the contrast between particle and solvent. Contrast denotes the excess scattering length density, i.e. the difference of electron density between solute and solvent. Since the incident beam interacts with the electron density, the scattering length is a measure for the intensity of the secondary wave and the difference between scattering length of the protein and the medium describes the contrast between sample and background. Following normalization for buffer background one obtains an intensity profile corresponding to the scattering of one particle. Since the technique measures the sample in its native state, i.e. in solution, the resulting scattering curve is an average over all possible particle orientations. From the thus obtained scattering curve a number of parameters (maximal dimension, particle volume, radius of gyration) can be derived directly, while calculation of the molecular shape needs some more elaborate procedures. The Fourier transform of the 
scattering curve gives the distance distribution function, which shows the abundance of certain distances $(r)$ occurring within the particle (Figure $8 \mathrm{~B}$ ). Clearly, this function depends on the particle's shape and one can derive the maximal dimension of the particle (Dmax). From the linearization of the scattering curve (Guinier plot; $\ln (I(q))$ vs. $q^{2}$; Figure $8 \mathrm{C}$ ) one can not only observe potential particle aggregation, but also read out $\mathrm{I}(0)$ (the scattering at zero angle; $y$ intercept of the Guinier-plot) and Rg (the radius of gyration; slope of the Guinier-plot).Obviously, $\mathrm{I}(0)$ cannot be measured directly, but is only an approximation for which's determination the measurement of very small angles is beneficial. To allow even at this small angles a clear distinction between the direct beam and the scattered one, a highly focused beam, as it is available at synchrotron sources, is a prerequisite. This intensity of the scattered beam at zero angles depends on the particle concentration and the particle volume. Thus, if the sample concentration is measured carefully one can calculate the particle's volume from I(0). Assuming that the specific density of e.g. a protein is always the same, calculation of the molecular weight is possible based on this particle volume.

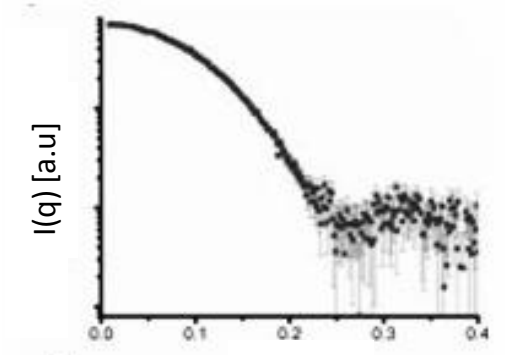

A
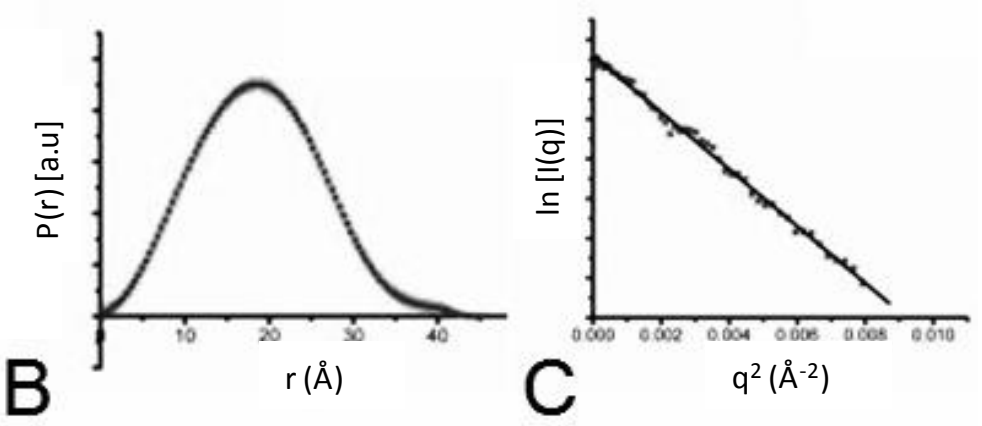

Figure 8: Data obtained directly from the SAXS-experiment. A Scattering curve of the sample corrected with scattering curve of the buffer $B$ The distance distribution function is obtained from $A$ by Fourier Transformation and represents a histogram of distances observed within the molecule. Besides other data, this function contains information about the maximal particle dimension (ca. $43 \AA$ for this example). C Guinier-Plot of $A$ is utilized to confirm that no aggregation occurs. Additionally, one can directly read out the scattering at zero angle (I(O); intercept with the y-axis) and the radius of gyration (Rg; slope of the function). Figures are taken with permission from ref (Jacques and Trewhella, 2010).

Additionally, more sophisticated algorithms allow calculating a particle envelope, which's theoretical scattering curve fits the experimentally observed one. There are several algorithms and programs available and the most important ones are collected in the program suite ASTAS from EMBL Hamburg. The principle of the algorithm implemented in the software DAMMIN and DAMMIF is outlined briefly. The scattering curve yields directly information on the maximal diameter of the investigated particle. Therefore, it is possible to define a sphere, which can completely enclose the investigated particle, as search volume and fill this volume with $\mathrm{M}$ beads that have a diameter $d$, with $d<<$ Dmax. Subsequently, each of this smaller spheres is assigned to be part of the particle (value=1) or the solvent (value=0). With this assignment the whole structure is described by a string of $M$ characters. Knowing that from every assumed shape a 
theoretical scattering curve can be calculated, one can compare the curve of the assumed shape with the experimental data and use the difference between these data to guide the process of $a b$ initio prediction of a molecular shape that perfectly describes the observed scattering curve. A very important aspect of a SAXS-experiment and especially of the derived ab initio structures is that a three dimensional molecular shape is derived from a one dimensional curve. Thus, the risk of overinterpreting the data and misleading results is given, especially since often a multitude of potential shapes will yield the observed scattering curve. Keeping this in mind, one will realize that the assurance of measuring pure, monodisperse samples is a necessary prerequisite, but not a sufficient criterion, for reliable interpretation of SAXS data. Therefore, a subsequent evaluation of the obtained model with other experimental data should be mandatory.

\subsection{Electron Paramagnetic Resonance and Double Electron Electron Resonance}

Electron paramagnetic resonance (EPR) is a spectroscopic technique to obtain information about the magnetic properties of electrons. For a more comprehensive description of this kind of spectroscopy, the reader is referred to literature (Lottspeich et al., 2006; Corvaja et al., 2009), but the basic principle will be outlined here: Magnetism is always associated with the kinematics of a charged particle. Keeping the electron-spin itself or the angular movement of the electron in a given orbital around an atom's nucleus in mind, one can easily see that an electron can be referred to as the world's smallest magnet. While typically atoms and molecules obey Hund's rule and have no magnetic properties, due to paired electrons with magnetic spins cancelling out each other, some transition metals have unpaired electrons in their $d$-shells and radicals have generally an unpaired electron giving rise to an observable magnetic moment. The principle of an EPR-experiment is that the magnetic moments of those electrons are split within an outer magnetic field (Zeeman Effect). A single electron has two magnetic states $m_{S}= \pm 1 / 2$ and thus the electrons are aligned within the applied magnetic field in either parallel or antiparallel orientation, with the latter having a higher energetic state than the parallel aligned electron. According to Zeeman's law the energy difference of both orientations $(\Delta E)$ is proportional to the effective magnetic field $\left(B_{\text {eff }}\right)$, meaning the higher the magnetic field is, the higher the splitting between both energetic states:

$\Delta E=\mu^{*} g^{*} B_{\text {eff }} ;$ with: $\mu$ - Bohr magneton and g- Landé $g$-factor

The macroscopic magnetic property of the compound is consequently proportional to the population difference between the two spin states. This population difference in turn is described by a Boltzmann distribution and is obviously inversely proportional to the temperature and proportional to the effective magnetic field. To probe the specific energetic difference 
between both states, which is characteristic for certain compound's properties described later on, the transition between both states is enabled by resonant absorption of microwaves:

$\Delta E=\mu_{B}{ }^{*} g^{*} B_{\text {eff }}=h * v ;$ with: $h$ - Planck constant and $v$ - the microwave frequency matching $\Delta E$

For elucidation of the resulting spectrum it is important to know that the effective magnetic field is not equal to the applied magnetic field $\left(B_{\text {appl }}\right)$, but is the sum of this magnetic field and a compound's internal magnetic field $\left(B_{\text {int }}\right)$ :

$B_{\text {eff }}=B_{\text {appl }}+B_{\text {int }}$

This internal magnetic field is caused by the following contributions:

I) Spin-orbit-coupling; magnetic field caused by the angular moment of the electron's movement around the nucleus

II) Spin-spin-coupling; magnetic field caused by additional unpaired electrons

III) Magnetic field of associated nuclei, this interaction causes a splitting called hyperfineinteraction. Often, this hyperfine-interaction can only be resolved at high magnetic fields and contains information on the atomic structure around the detected paramagnetic species.

This compound specific internal magnetic field can be described by a g-value deviating from that of the free electron. Caused by anisotropic geometry of the electron orbital, this g-value shows also an anisotropic behaviour and consequently, within a powder-sample or a frozen liquid three g-values can be observed and deliver additional information about the molecular geometry. In a single crystal these spatial dependencies can be resolved and in a liquid solution the fast tumbling of the molecule leads to a single, time-averaged and broadened g-value. Following this deviation the g-value is a specific fingerprint of the investigated compound and its tensor can be utilized to obtain information not only about the paramagnetic species, but also about the rhombicity of the electron-orbital and the nature of neighbouring nuclei. The most obvious way to obtain EPR-data is to measure a continuous wave (cw)-spectrum. That is to keep the microwave frequency fixed and continuously sweep the magnetic field over a given range of values.

Obviously a wealth of information can be extracted from such a cw-EPR-spectrum, but interpretation is complicated by this vast amount of parameters. To overcome these limitations, pulsed EPR can be utilized, with different pulse sequences yielding spectra with distinct information and the possibility to resolve several signal details that would give rather an inhomogeneous broadened signal than resolved information in a cw-experiment. The principle of these experiments is that electron spins aligned parallel or antiparallel with an outer magnetic field are rotated out of their equilibrium state by application of a short resonant microwave pulse, whereby the pulse length or the pulse intensity can be utilized to adjust the angle by 
which the spins are rotated. By definition a $\pi / 2$-pulse is used to rotate the magnetization from the z-axis of a Cartesian coordinate system into $x$-y-plane. Subsequently, two different interactions of the spins with their surrounding will lead to a decay of magnetisation within the $x-y$-plane. The spin lattice relaxation $\left(T_{1}\right)$ describes relaxation of the magnetization back to the $z-$ axis (i.e. to an equilibrium state) and the transverse relaxation $\left(T_{2}\right)$ describes the fanning out of magnetization in the $x-y$-plane. This transverse relaxation can be reversed by an additional pulse, causing a $180^{\circ}$ rotation of the magnetization and thus leading to refocusing of magnetization and producing an echo after the same time-delay placed between both pulses. The produced spin-echo is termed "Hahn-echo" and the described pulse-sequence is one of the simplest that can be utilized. From the large amount of pulse-sequences that a sample can be subjected to, a technique utilized in this study to measure intramolecular radical distances should be pointed out:

\subsubsection{DOUBLE ELECTRON-ELECTRON-RESONANCE (DEER)}

DEER, sometimes also referred to as pulsed electron-electron double resonance (PELDOR), is a pulsed EPR-technique that gives information about dipolar-interactions (Reginsson and Schiemann, 2011). This information can for instance give rise to knowledge on the distance of two independent paramagnetic species in a molecule. Since performed in frozen solution, but yielding precise distances and distance distributions between two paramagnetic labels of a macromolecule, it is a technique complementary to fluorescence approaches that yield rather imprecise real-time data of molecular distances and dynamics.

The presence of a second paramagnetic centre within the same molecule gives rise to an internal magnetic field that will result in a splitting of the EPR-signal. Since the magnitude of this dipoledipole interaction depends on the distance between the dipoles, in a continuous wave-spectrum the splitting will be resolved only for small distances (up to $2 \mathrm{~nm}$ ), while it leads to an inhomogeneous signal broadening for larger distances. To overcome this limitation the pulsed method DEER is utilized. The pulse sequence is designed in a way that allows specific detection of dipolar-couplings, while contributions of hyperfine-splitting are suppressed. Exactly, the dipolar coupling is described by the following equation:

$v_{\text {dd }}(r, \theta)=\frac{\mu * g 1 * g 2 * \mu B^{2}}{4 * \pi * h * r^{3}} *\left(3 \cos ^{2} \theta-1\right)$

where $v_{d d}$ is the dipolar coupling, $\mu$ is the vacuum permeability, $g_{1}$ and $g_{2}$ are the g-values of the respective paramagnetic species, $\mu_{\mathrm{B}}$ is the Bohr magneton, and $\mathrm{h}$ is Planck's constant. The important parameters in this equation are: $\theta$-the angle between the vector of the applied magnetic field and the vector spanning the distance between both dipoles and $r$-the distance between both dipoles, i.e. the desired information. This dipolar coupling is measured as modulation of the amplitude of a refocused echo. The modulation is caused and depends on the 
time of a pump-pulse resonant to the coupled dipole and accomplished at varying time-points $t$, swept between the first and the second $\pi$-pulse of the observer-sequence (Figure 9). The pulse sequence is started with a $\pi / 2$-pulse rotating the magnetization of the spins in resonance with the observe-pulse frequency to the $x-y$-plane. In this plane the transverse relaxation will lead to a fanning-out of the magnetization. By application of a $\pi$-pulse after $\tau_{1}$, the magnetization vectors will be rotated by $180^{\circ}$ and the fanning-out is reversed. Consequently, the magnetization is focused again and will produce a Hahn echo after $2 * \tau_{1}$. In the following evolution time $\left(\tau_{2}\right)$, the pump pulse is applied and the signal is eventually detected by application of a final $\pi$-pulse at the observer frequency.

Figure 9: Pulse-sequence applied for a four-pulse DEER-measurement. While three pulses are utilized at the Observe:

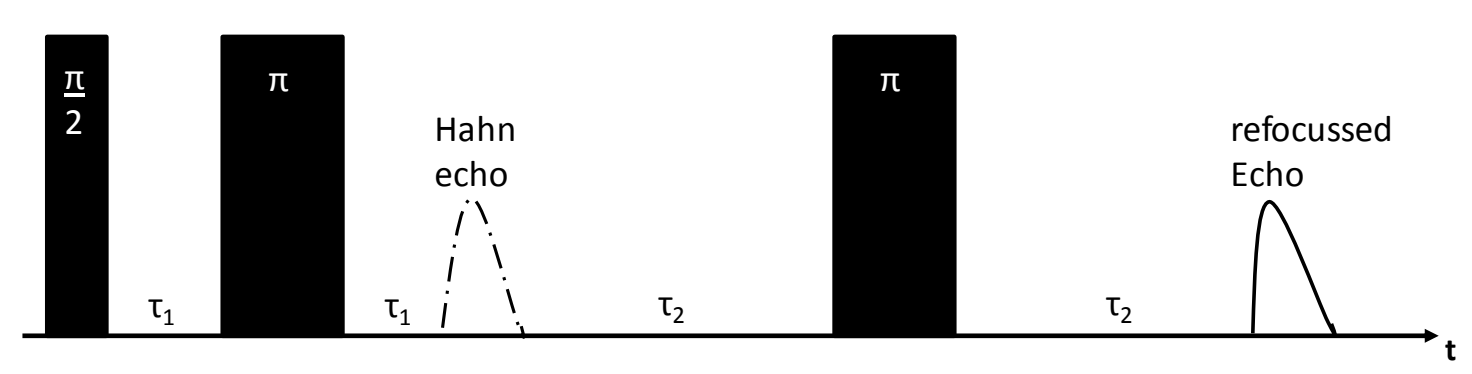

Pump:

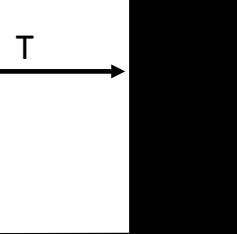

resonance frequency of one of the paramagnetic species to produce a refocused echo, the fourth is applied at a frequency resonant to the (putatively) dipolar-coupled paramagnetic species ("pump"). By modulating the time of this pump pulse application between $2 * \tau_{1}$ and $2 * \tau_{1}+\tau_{2}$ the refocused echo of the observed dipole is modulated (assuming that both dipoles are coupled). The frequency of this modulation is the dipolar coupling and thus contains information on the distance between both dipoles.

The resulting signal in the time domain represents contributions of intramolecular and intermolecular dipolar couplings. Subtraction of the randomly distributed intermolecular distances, which give a monoexponential decay, leads to a signal purely influenced by the intramolecular distances. To extract the desired distance distribution from the resulting signal several approaches ranging from a simple Fourier transformation to Tikhonov regularization can be pursued. In order to detect long distances, one has to assure that the dipolar evolution time $\tau_{2}$ is long enough to cover a full oscillation. Yet, the upper limit of this time window is restricted by the system's transverse relaxation $\left(T_{2}\right)$. Although this relaxation time might be prolonged (e.g. 
by addition of glycerol or using deuterated buffer), this limitation commonly determines the upper limit of detectable distances $(\sim 8 \mathrm{~nm})$.

\subsection{Kinetic Isotope Effects (KIE)}

To assess the kinetic contribution of a discrete step of bond fission within a complex scheme of reaction steps to an overall-reaction, the measurement of KIEs can be utilized. By labeling of the substrate with a heavy isotope, the zero-point energy of the bond is reduced and hence, assuming a fixed temperature, the rate of this microscopic reaction step is lowered, due to an increased activation energy. Caused by the largest possible relative mass difference, this effect is most pronounced for labeling with the derivatives of hydrogen. The result of such an experiment is usually represented as ratio of the rate constant for the light isotopologue (e.g. $k_{H}$ ) divided by the rate constant for the reaction with the heavy isotopologue (e.g. $k_{D}$ ), i.e. typically this fraction is bigger than one. Since the inherent decrease of the bond fission rate is caused by a defined change of the bond's reduced mass, one can calculate a range of fold-difference which encloses the experimentally expected value. Nevertheless, a quantum mechanical effect named hydrogen tunneling and describing the ability of the bond breaking process to transit through a lower energy pathway, which is forbidden in classical mechanics, can lead to a significantly higher $k_{H} / k_{D}$, since this tunneling can occur more likely for hydrogen than for the heavier isotopes.

By investigating whether this intrinsic KIE of a single reaction step is reflected in the macroscopically observed kinetics, one can deduce information about the interplay of discrete reaction steps, how they kinetically make up the overall reaction and whether the investigated step is rate limiting. For a more comprehensive description of this method, the reader is referred to literature (Ranaghan and Mulholland, 2010).

\subsection{Measuring redoxpotentials by direct electro chemistry}

Typically, electron transport through the protein to the active site of a redox-active protein is the limiting factor for direct electrochemistry of proteins (Léger and Bertrand, 2008). Direct means in this context that an electrode and not a redox partner is source or sink for the electrons delivered to/from the protein's active site. To overcome this limitation, either promoters or mediators can be applied (Hu, 2001). Promoters are substances that allow the protein of interest to form films on the electrode surface and thus ease the electron transfer. In contrast, mediators are low molecular weight redox-active substances that can carry electrons from the electrode to the active site of the investigated protein. Thus, this kind of voltametry is, strictly spoken, not a direct one. It was reported for several heme proteins that the best signal can be obtained by a combination of pyrolytic graphite electrode and DDAB (ㅁidecyldimethylammonium bromide) as promoter (Hu, 2001). This substance will form a hydrophobic layer on the electrode surface and 
then can eventually mimic the situation of redox transfer, occurring if the protein is bound to a biological membrane (Hu, 2001). The experimental approach to immobilize the redox active protein on the surface of an electrode is also known as "protein film voltammetry" and can be utilized to gain information not only about the redoxpotential but also about the kinetics of electron transfer (Armstrong, 2009).

Nevertheless, interpretation of the potentials obtained for redox-proteins immobilized on surfaces and their physiological relevance remains hard. It was shown for various systems that the midpoint potential measured for immobilized proteins and the redox potential of the protein in solution has not necessarily the same value (Verhagen et al., 1995). Also the chosen surfactant to immobilize the protein can have a drastic effect on the observed value ( $\mathrm{Hu}, 2001$; Johnson et al., 2002; Udit et al., 2006). This implies that for each investigated redox-active protein various surfactant/ electrode-combinations have to be examined, the measured values always have to be stated in combination with the utilized immobilization chemistry and interpretation of the measured data has to be done carefully.

\subsection{Identification of oxylipins by $\mathrm{LC} / \mathrm{MS}^{2}$}

LC-MS' , i.e. a triple quadrupole with electron spray ionization coupled to Reverse-phase HPLC, was described as ideal analytical tool for identification and quantification of oxylipins (E H Oliw et al., 1998; Nilsson et al., 2010). A typical MS/MS-experiment to detect oxylipins is performed in negative mode and thus deprotonated fatty acid derivatives are detected. The fragmentationpattern contains only a limited number of highly informative peaks. Besides a peak corresponding to loss of water, the analytes are characteristically fragmented at carbons functionalized with hydroxyl-groups. Elucidation of the oxylipin's structure can be further facilitated by enzymatic conversion of fatty acid isotopologues labeled with stable isotopes (e.g.

${ }^{13} \mathrm{C}$-and ${ }^{2} \mathrm{H}$-labeled fatty acid). 
For this study, the products produced by PpoA from oleic and linoleic acid are of interest. These oxidized C18 fatty acids are (ranged according their typical abundance in fatty acid conversions by wild type enzyme):

Products derived from oleic acid (IUPAC: (9Z)-Octadec-9-enoic acid)):

- 5,8-Dihydroxy oleic acid (5,8-DiHOME; IUPAC: (9Z)-5,8-Dihydroxy-octadec-9-enoic acid)

- 8-Hydroperoxy oleic acid (8-HPOME; IUPAC: (9Z)-8-Hydroperoxy-octadec-9-enoic acid)

- 8-Hydroxy oleic acid (8-HOME; IUPAC: (9Z)-8-Hydroxy-octadec-9-enoic acid)

Side products:

- 8-Keto oleic acid (8-KOME; IUPAC: (9Z)-8-Oxo-octadec-9-enoic acid)

- 6,8-Dihydroxy oleic acid (6,8-DiHOME; IUPAC: (9Z)-6,8-Dihydroxy-octadec-9-enoic acid)

- 8,11-Dihydroxy oleic acid (8,11-DiHOME; IUPAC: (9Z)-8,11-Dihydroxy-octadec-9-enoic acid)

- 8-Hydroxy-9,10-epoxy-octadecanoic acid

- 10-Hydroxy-8,9-epoxy-octadecanoic acid

Products derived from linoleic acid (IUPAC: (9Z,12Z)-Octadeca-9,12-dienoic acid):

- 5,8-Dihydroxy linoleic acid (5,8-DiHODE; IUPAC: (9Z,12Z)-5,8-Dihydroxy-octadeca-9,12dienoic acid)

- 8-Hydroperoxy linoleic acid (8-HPODE; IUPAC: (9Z,12Z)-8-Hydroperoxy-octadeca-9,12dienoic acid)

- 8-Hydroxy linoleic acid (8-HODE; IUPAC: (9Z,12Z)-8-Hydroxy-octadeca-9,12-dienoic acid)

Side products:

- 8-Keto linoleic acid (8-KODE; IUPAC: (9Z,12Z)-8-Oxo-octadeca-9,12-dienoic acid)

- 6,8-Dihydroxy linoleic acid (6,8-DiHODE; IUPAC: (9Z,12Z)-6,8-Dihydroxy-octadeca-9,12dienoic acid)

- 8,11-Dihydroxy linoleic acid (8,11-DiHODE; IUPAC: (9Z,12Z)-8,11-Dihydroxy-octadeca-9,12dienoic acid)

- (12Z)-8-Hydroxy-9,10-epoxy-octadec-12-enoic acid

- (12Z)-10-Hydroxy-8,9-epoxy-octadec-12-enoic acid

Figure 10 summarizes the LC-MS ${ }^{2}$ spectra of the oxylipins derived from linoleic acid. The corresponding oleic acid derived products exhibit basically the same spectrum. Merely the fragment containing the $\mathrm{C}_{12}-\mathrm{C}_{13}$ bond will have a mass/charge-ratio increased by 2 . 

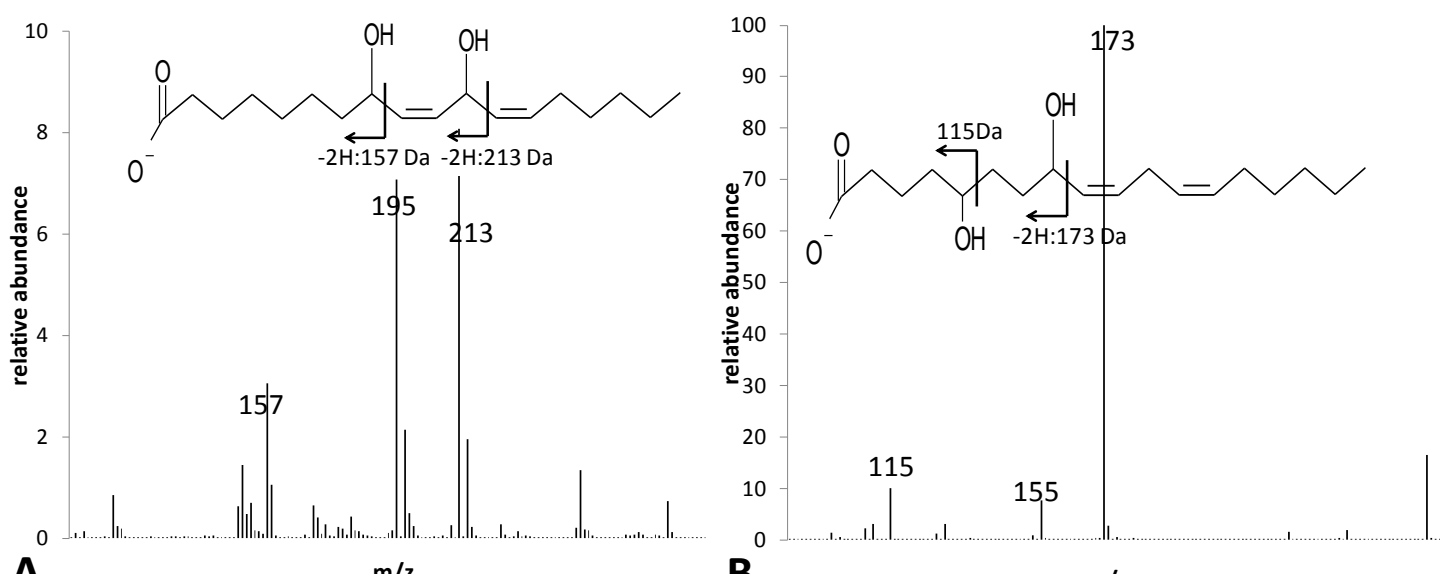

A

$\mathrm{m} / \mathbf{z}$

B

$\mathrm{m} / \mathbf{z}$
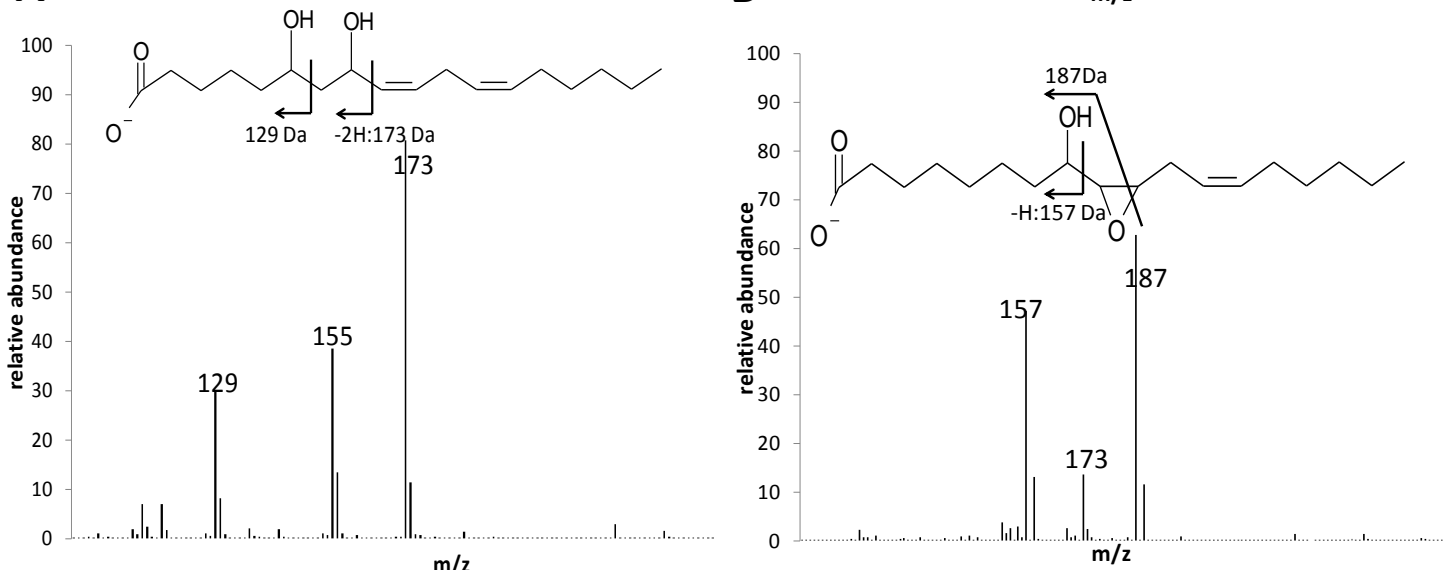

C

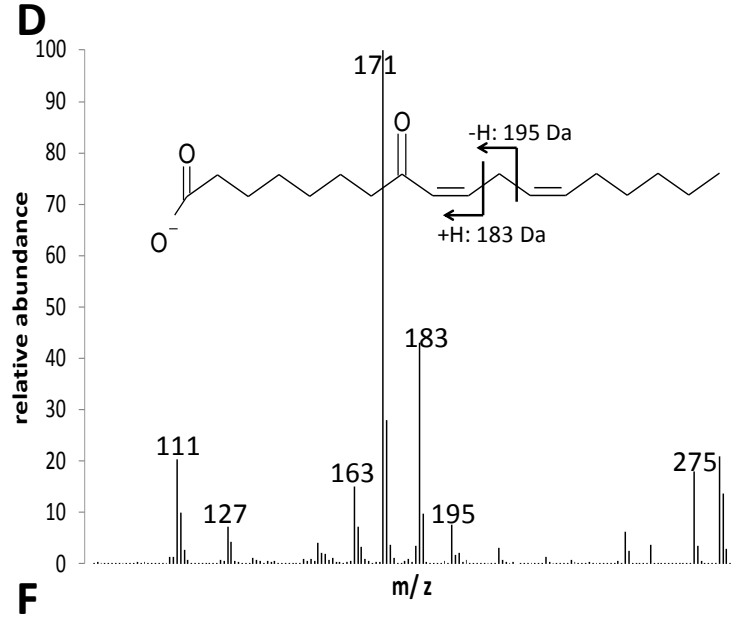

E

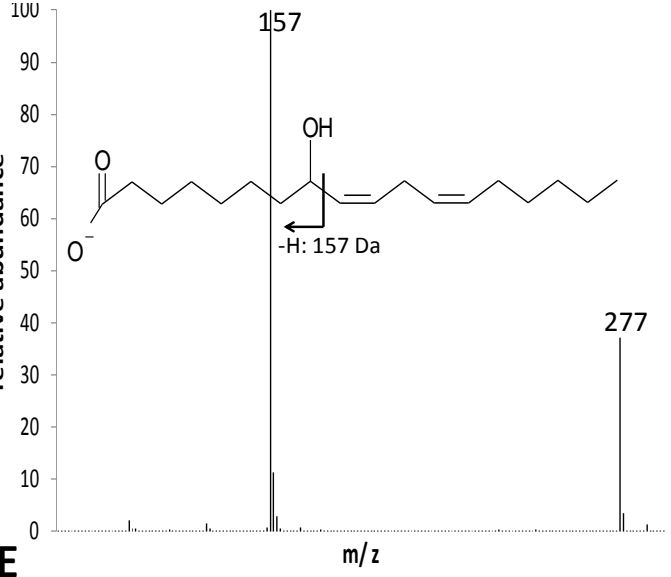

Figure 10: $L C-M S^{2}$-spectra of oxylipins formed by fungal Ppo-enzymes. The spectra are ordered in their range of elution from the RP-HPLC system described in Materials and Methods. A Fragmentation of 8,11DiHODE; a reference-spectrum can be found in (Jernerén et al., 2010) B Fragmentation of 5,8-DiHODE; a reference-spectrum can be found in (Garscha and Ernst H Oliw, 2007) C Fragmentation of 6,8-DiHODE; the 129 Da-Fragment can be unequivocally identified by utilization of ${ }^{13} \mathrm{C}_{18}$-linoleic acid $\boldsymbol{D}$ Fragmentation of 8hydroxy-9,10-epoxy-12Z-octadecenoic acid; a reference-spectrum can be found in (E H Oliw et al., 1998). Note that due to Payne rearrangement, the fragmentation pattern of 10-hydroxy-8,9-epoxy-12Zoctadecenoic acid is basically the same (Ernst $H$ Oliw et al., 2006). E Fragmentation of 8-HODE; a reference-spectrum can be found in (Garscha and Ernst $H$ Oliw, 2007) $F$ Fragmentation of 8-KODE; a reference-spectrum can be found in (E H Oliw et al., 1998). 


\section{RESULTS}

\subsection{Enzyme preparation and quality control}

The prerequisite for the intended biochemical and biophysical characterization of PpoA are expression and purification processes that enable to generate high amounts of homogeneous enzyme. Wild type enzyme and variants were expressed and purified as described in a previous study (Brodhun et al., 2009) and specified in the experimental part (Section 8.1.12). While variants for which only the product pattern was determined (Met791Leu; Met791Leu/Ala792Val; Met791Leu/Ala792Met; Phe795Leu; Phe799Tyr; Phe799Trp; Phe795Leu/Phe799Leu; Leu1009Glu), were not purified, but the cell pellet was only lysed, variants that were further characterized were purified to homogeneity. The quality of the resulting enzyme batch was controlled by a combination of SDS-PAGE, UV-Vis-and CDspectroscopy (data not shown). Except for the Phe799Leu- and Phe799Met-variant, CD-spectra were measured once for every variant in order to assure that the overall protein fold was not perturbed by the mutation (near UV-spectra) and that integrity of the heme cavity was not affected (far UV-spectra). Enzyme concentration was determined spectroscopically, using a theoretically derived specific extinction coefficient of $\varepsilon_{280}=126000 \mathrm{M}^{-1} \mathrm{~cm}^{-1}$. UV-Visspectroscopy was also used as major tool to assess enzyme homogeneity, since the ratio of heme absorption at the soret-peak and at $280 \mathrm{~nm}$ equals one for a PpoA-preparation free of (protein) impurities. When the ratio $A_{410} / A_{280}$ differed from unity, SDS-PAGE was employed to check the protein purity and determine whether the observed deviation is caused by a protein contamination of the sample or reduced heme occupancy, caused by the mutation. The QCparameters for all enzyme batches except Asn887Val and Phe799Leu were consistent with wild type enzyme. For these two variants the soret-band showed reproducibly a significant reduced absorption, in comparison to wild type enzyme. For the Asn887Val-variant $\mathrm{A}_{410}$ was reduced by approximately $30 \%$, while the Phe799Leu-variant exhibited about $60 \%$ of the soret-absorption of wild type enzyme. Yet, SDS-PAGE indicated a homogeneous enzyme preparation. To attribute the thus concluded most likely lowered heme occupancy of the enzyme to one of the two domains, cw-EPR-spectra of the purified enzyme were recorded at X-band (in cooperation with Alistair Fielding, MPI for Biophysical Chemistry, Goettingen; Data not shown). While the highspin heme showed the same abundance as for a wild type sample with the same proteinconcentration (as judged by an equal $A_{280}$ ), the low-spin heme signal was reduced by approximately $40 \%$ for the Asn887Val-variant and $80 \%$ for the Phe799Leu-variant. According to the assignment by Fielding et al. (Fielding et al., 2011), this observation could be interpreted as a lower heme-occupancy of the cytochrome P450 domain of PpoA, caused by the conducted amino acid exchange. Qualitatively, the g-tensor of the Phe799Leu-variant was comparable to wild type, but in the Asn887Val-variant a changed $g_{1}$ indicated a perturbed coordination of the heme. 


\subsection{Attempts to crystallize the enzyme}

\subsubsection{REFINEMENT OF A PREVIOUSLY IDENTIFIED PROMISING CONDITION}

In a previous crystallization trial, Florian Brodhun could identify a precipitant combination that yielded spherulites and thus could putatively serve as initial condition for further refinement. To refine this condition (initially: $1.3 \mathrm{M}\left(\mathrm{NH}_{4}\right)_{2} \mathrm{SO}_{4} ; 200 \mathrm{mM} \mathrm{Li}\left(\mathrm{SO}_{4}\right) ; 100 \mathrm{mM}$ Tris- $\mathrm{HCl}, \mathrm{pH}$ 7.6) precipitant and protein concentrations were systematically varied and the effect of several additives and the temperature on crystallization was investigated. The detailed process of successive refinement is outlined briefly:

I) Systematic variation of precipitant-concentration at a fixed protein concentration ( $15 \mathrm{~g} / \mathrm{l})$ : Salt concentrations were varied in the range of: 1.6 to 7.0 $M$ ionic strength, with the single salts systematically varied in the range of 0.1 to $1.4 \mathrm{M}$.

II) Variation of the protein concentration in the range of 3 to $15 \mathrm{~g} / \mathrm{l}$.

III) Addition of additives (1,6-hexanediol, phenol, glycerol, EDTA, DTT)

IV) Vapor diffusion at 4 and $20^{\circ} \mathrm{C}$

V) Streak-seeding with so far obtained, small spherulites.

Despite all efforts no improvements in spherulite size or shape could be achieved (Figure $11 \mathrm{~A}$ ).

\subsubsection{NEW SCREENING FOR CONDITIONS EVENTUALLY YIELDING CRYSTALS}

Since the previously identified initial hit could not be optimized to give a crystal suitable for data collection, a new initial screen with wild type enzyme was performed. Seven distinct 96-well plates representing the most common used conditions for crystallization were utilized with different enzyme preparations at various temperatures. From this screening only nine conditions were found to yield spherulites that might be considered as initial 'hit' for a further refinement. While the vast majority of the investigated conditions yielded either precipitate or remained clear, these nine wells contained aggregated enzyme that served as initial condition for refinement. From the given 'hits' the best (Figure $11 \mathrm{~B}$ ) was chosen and subjected to a thorough refinement as outlined in 4.2.1. Again no improvement of spherulite size or form was achieved. 


\subsubsection{Systematic test OF Divalent Cations}

Since screening of more than 5000 conditions did not yield successful results, an alternative to sparse matrix screens was considered (Bergfors, 1999, Chapter 10). The idea of this kind of rational screen was to adjust the $\mathrm{pH}$ to a value at which the protein will be negatively charged. At this state, positively charged metal ions might act as counter-ions and mediate crystal contacts between single protein-molecules. Using this rational, following salt precipitants are supposed to yield satisfying results: ammonium sulfate, ammonium phosphate, ammonium citrate, ammonium acetate, magnesium sulfate and calcium chloride. From theoretical calculations considering the number of charged amino acids, one can find that the net-charge of PpoA is supposed to be negative at $\mathrm{pH}$ 8.5. At this $\mathrm{pH}$ imidazole and TRIS are substances that exhibit adequate buffer capacities. Screenings with $20 \mathrm{mg} / \mathrm{ml}$ of wild type enzyme at this $\mathrm{pH}$ utilizing a matrix with variations in the employed buffer, the 6 aforementioned precipitants in varying concentration, a multitude of possible divalent cations ( $\mathrm{Ni}(\mathrm{II}) \mathrm{Cl}_{2}, \mathrm{ZnCl}_{2}, \mathrm{Fe}(\mathrm{II}) \mathrm{SO}_{4}, \mathrm{MgCl}_{2}$, $\mathrm{CaCl}_{2}$ and $\mathrm{CuCl}_{2}$ ) and several additives, the most promising results have been obtained in imidazole buffer with ammonium sulfate as precipitant and either $\mathrm{ZnCl}_{2}$ or $\mathrm{CuCl}_{2}$ as metal ions (Figure $11 \mathrm{C}$ ). Although these initial hits seemed to be promising, a refinement to high quality crystals was not possible.
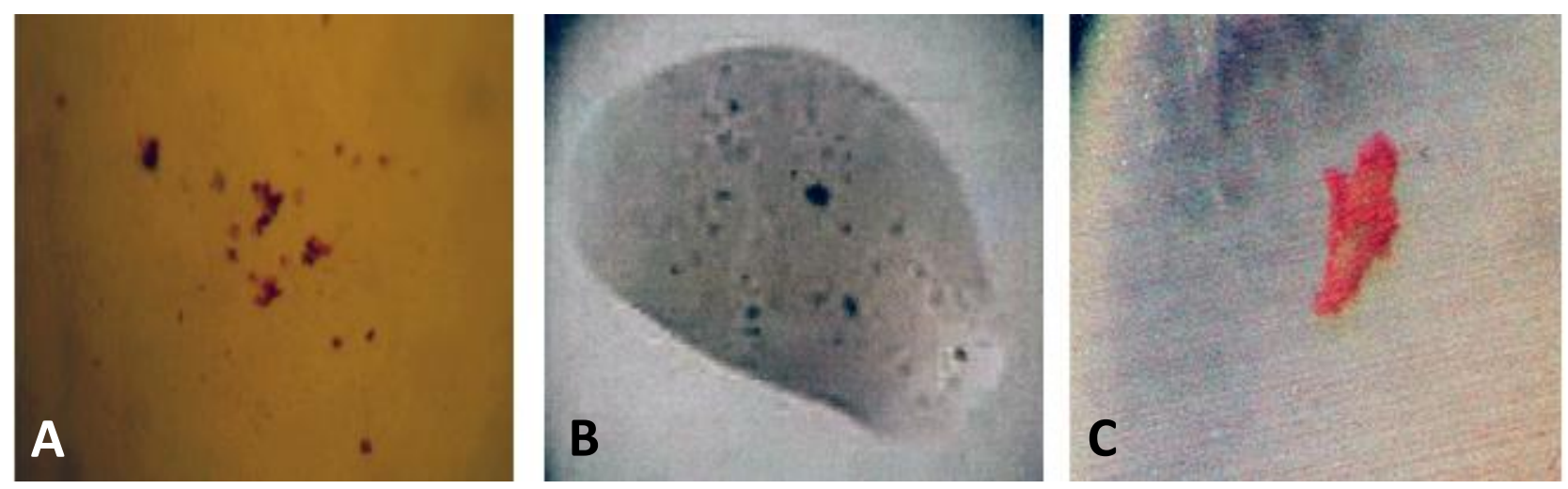

Figure 11: Results obtained for crystallization trials with the unmodified protein. $\boldsymbol{A}$ Refinement of a previously identified condition (( $\left.\mathrm{NH}_{4}\right)_{2} \mathrm{SO}_{4} ; \mathrm{Li}_{2}\left(\mathrm{SO}_{4}\right)$; Tris-HCl; Section 4.2.1) B Best hit in a new screen for a suitable condition (PEG 20000; Na-MES; Section 4.2.2) C Aggregated PpoA, obtained in a rationalized screen $\left(\left(\mathrm{NH}_{4}\right)_{2} \mathrm{SO}_{4} ; \mathrm{CuCl}_{2} ;\right.$ Section 4.2.3) 


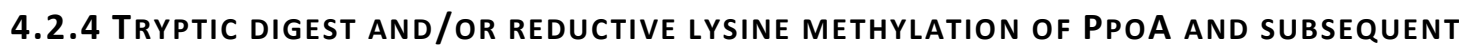 CRYSTAL SCREENS}

It is well known that several proteins have (not necessarily obvious) properties that prevent a successful crystallization. To possibly rescue a project aiming to crystallize such a protein, several protein modifications might be performed (see section 3.1). Two of these various methods have been utilized rather often and have reliably improved the ability to form crystals in several cases: In situ tryptic digest to remove flexible loops of the protein and methylation of surface exposed Iysines to overcome charge-charge repulsions (Kim et al., 2008; Wernimont and Edwards, 2009). Both methods were applied as described in literature and the resulting protein was subjected to a screening for suitable initial conditions as it is described for the wild type enzyme in section 4.2.2. However, the results indicated that the mentioned methods to modify the protein itself, did not improve the crystallizability of PpoA. Also a combination of both methods was not successful.

\subsubsection{GRAFIX AND SUBSEQUENT CRYSTAL SCREEN}

GraFix is an approach developed for 3D-cryo electron microscopy in order to overcome sample properties like polydispersity and flexibility that might negatively affect this kind of experiment (Kastner et al., 2008). The basic principle is that homogeneity of the purified protein sample is assured by ultracentrifugation in a density gradient. This process will select for mass differences and discriminate between distinct oligomeric states and incomplete particle assemblies. To stabilize the enriched protein state, a glutaraldehyde gradient in the tube is used to cross-link the protein, which leads to fixation of a given homogenous sample state. Additionally, this crosslinking agent is thought to be involved in stabilizing flexible parts of the protein by linking them to the stable protein core. These putative benefits of the GraFix approach make it an interesting method also for modification of protein (complexes) to be crystallized. Especially the oligomeric structure of PpoA seems to make it a valuable target for the GraFix approach. Moreover, GraFix was facultatively combined with limited trypsolysis to probe for potential synergistic effects. Figure 12 summarizes the approach, shows the characterization of the modified protein and presents the two most interesting results of the subsequent crystallization screens.

However, also this protein modification did not lead to a substantial increase in the amount of hits, i.e. interesting conditions that might be worth refinement. Nevertheless, two conditions showed very promising initial results (Figure $12 \mathrm{C}$ and D). Interestingly, these two conditions were identical and differed only in the incubation temperature. Thus, MPD in combination with imidazole buffer was identified as a promising condition for further refinements. As diverse other conditions with MPD did not yield spherulites, no other buffers or additional precipitants were chosen for initial optimization of the identified condition. For the refinement screen both, 
precipitant concentration and $\mathrm{pH}$, were systematically varied. The $\mathrm{pH}$ was adjusted to values from 6.05 to 8.05 over the rows of a 96-well block and the MPD-concentration was changed over the columns, starting with a concentration of $10 \%(w / v)$ to a concentration of $40 \%(w / v)$. Since the described initial refinement did not lead to an improvement of the spherulites formed, the matrix of screened parameters was enlarged and the effect of protein concentration and various additives was investigated. To possibly benefit from the effects of ligand binding the functional variant PpoA_Tyr374Phe (Brodhun et al., 2009) was employed, but, as also seen for the unmodified protein, a co-incubation of this variant with linoleic acid was rather counterproductive. Finally, further improvements of well behaved conditions were attempted by various seeding techniques. Nevertheless, again all efforts failed and no significant improvement or well diffracting crystals could be obtained (Figure 13).
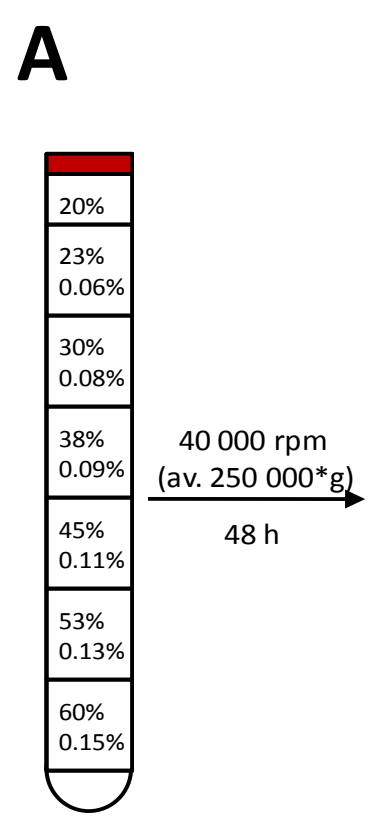
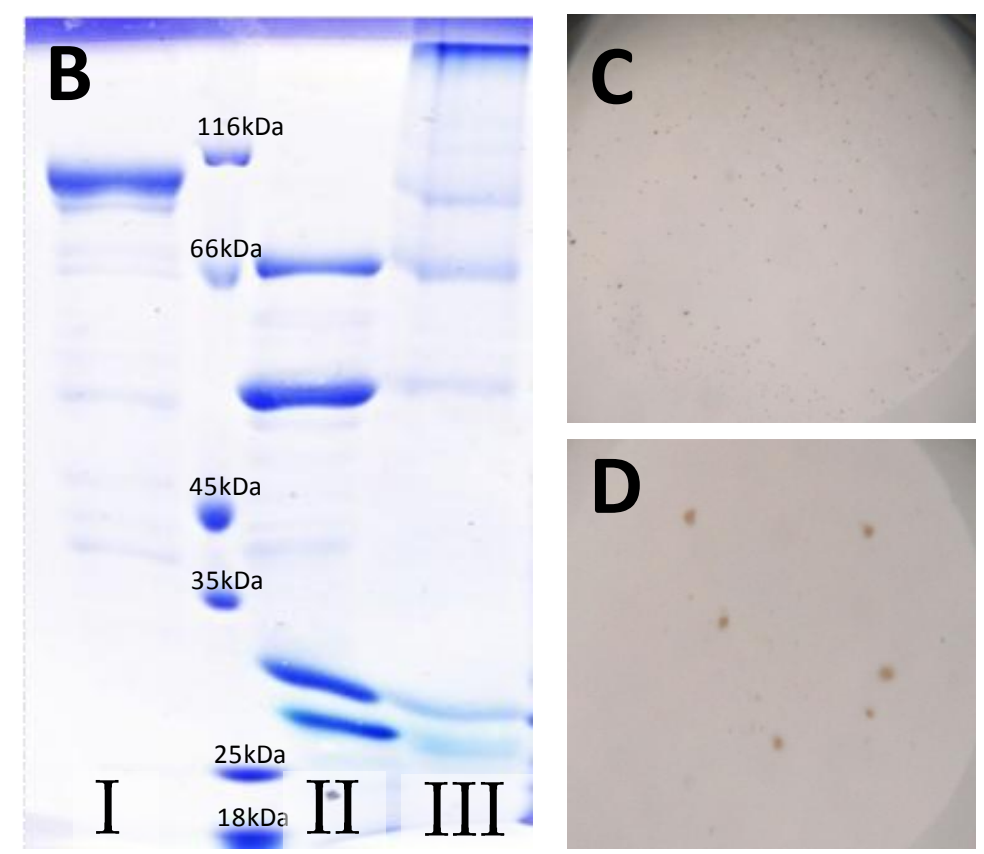

Figure 12: Modification of PpoA by tryptic digest and GraFix. A A discontinuous saccharose gradient (20 to $60 \%(w / v))$ with glutaraldehyde $(0.06$ to $0.15 \%(w / v))$ was utilized to homogenize and simultaneously cross-link a PpoA-sample. The sample was purified and digested as described in sections 8.1.12 and 8.1.15. Subsequently the enzyme (red) was subjected to the GraFix-gradient presented in A. B SDS-PAGE of a wild type standard (i.e. undigested PPoA; lane I), the digested enzyme after SEC (lane II) and after rescue from the GraFix-gradient (lane III). $\boldsymbol{C}$ The first of two interesting hits obtained upon crystallization screens with the modified protein. $250 \mathrm{nl}$ of $11 \mathrm{~g} / \mathrm{l}$ tryptic digested and glutaraldehyde cross linked wt-enzyme were mixed with $250 \mathrm{nl}$ reservoir ( $0.1 \mathrm{M}$ imidazole, $\mathrm{pH} 8 ; 35 \%(\mathrm{w} / \mathrm{v})$ 2-methyl-2,4-pentandiol) and stored for $14 \mathrm{~d}$ at $20^{\circ} \mathrm{C}$. D The second hit of the crystallization screen. $125 \mathrm{nl}$ of $11 \mathrm{~g} / \mathrm{l}$ tryptic digested and glutaraldehyde cross linked wt-enzyme were mixed with $250 \mathrm{nl}$ reservoir (0.1 M imidazole, pH 8; $20 \%$ (w/v) 2-methyl-2,4pentandiol) and stored for $14 \mathrm{~d}$ at $4^{\circ} \mathrm{C}$. 

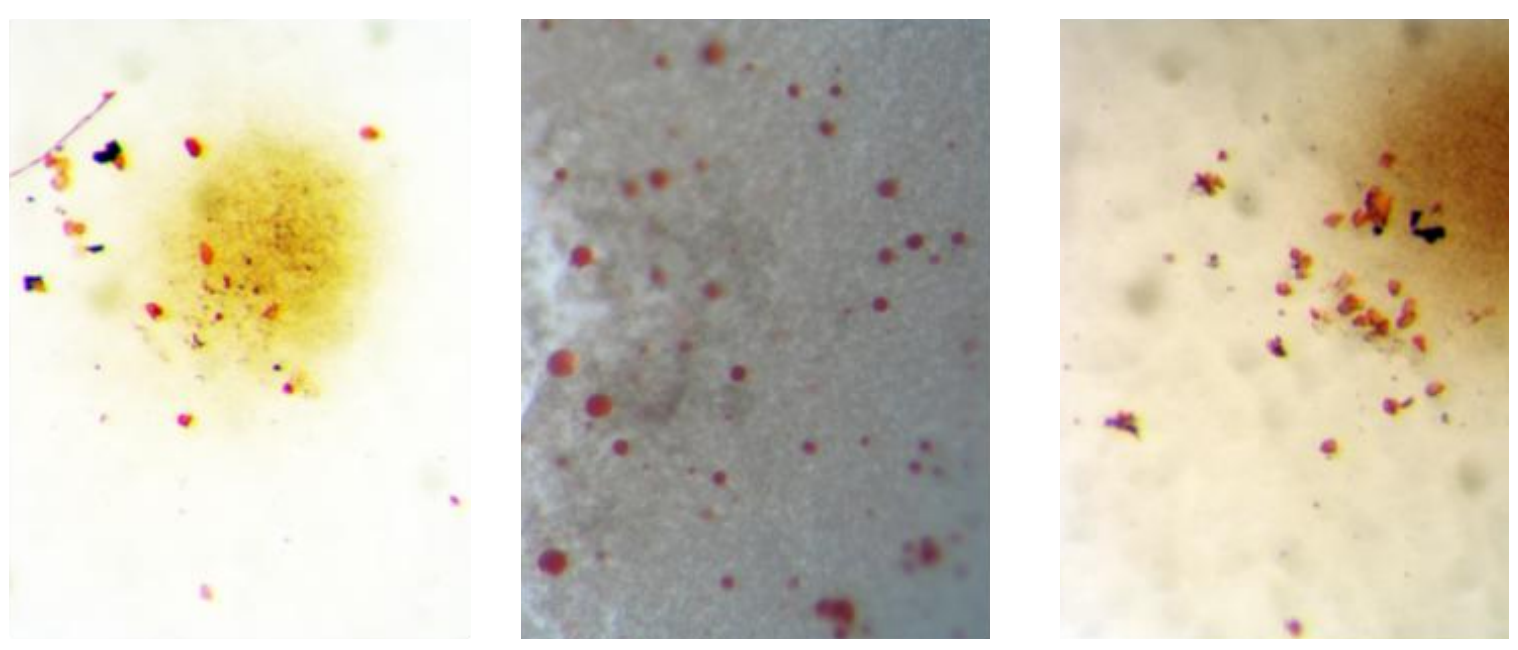

Figure 13: Spherulites obtained by refinement of crystallization conditions for the tryptic digested and GraFix modified enzyme in conditions with imidazole-buffer and MPD-precipitant.

The observed beneficial use of imidazole buffer might be explained by the known coordination of the imidazole ring to the heme of various P450s (Yeh et al., 2005). By this binding a conformational change from the opened enzyme-form to a closed and more rigid one might be accomplished (H. Li and Thomas L. Poulos, 1999). Visible spectra of the enzyme obtained in 20 mM HEPES buffer before and after addition of imidazole indicated that also in the P450-domain of PpoA a six-ligated iron complex with imidazole is formed (Figure 14). Nevertheless, it was shown for class III P450s that the coordination of imidazole to the heme is only weak (Yeh et al., 2005). In line with this, also quantitative imidazole binding studies with wild type enzyme and the His10004Ala-variant, in which the P450-heme is absent (Brodhun et al., 2009), did not lead to an unequivocal assignment of the heme-domain in which this complex is formed (See also section 4.4.4). Taken together, the results of the spectral titration indicate that imidazole might bind to the cysteine as well as the histidine coordinated heme and could eventually induce conformational changes favoring crystallization in both PpoA-domains. 


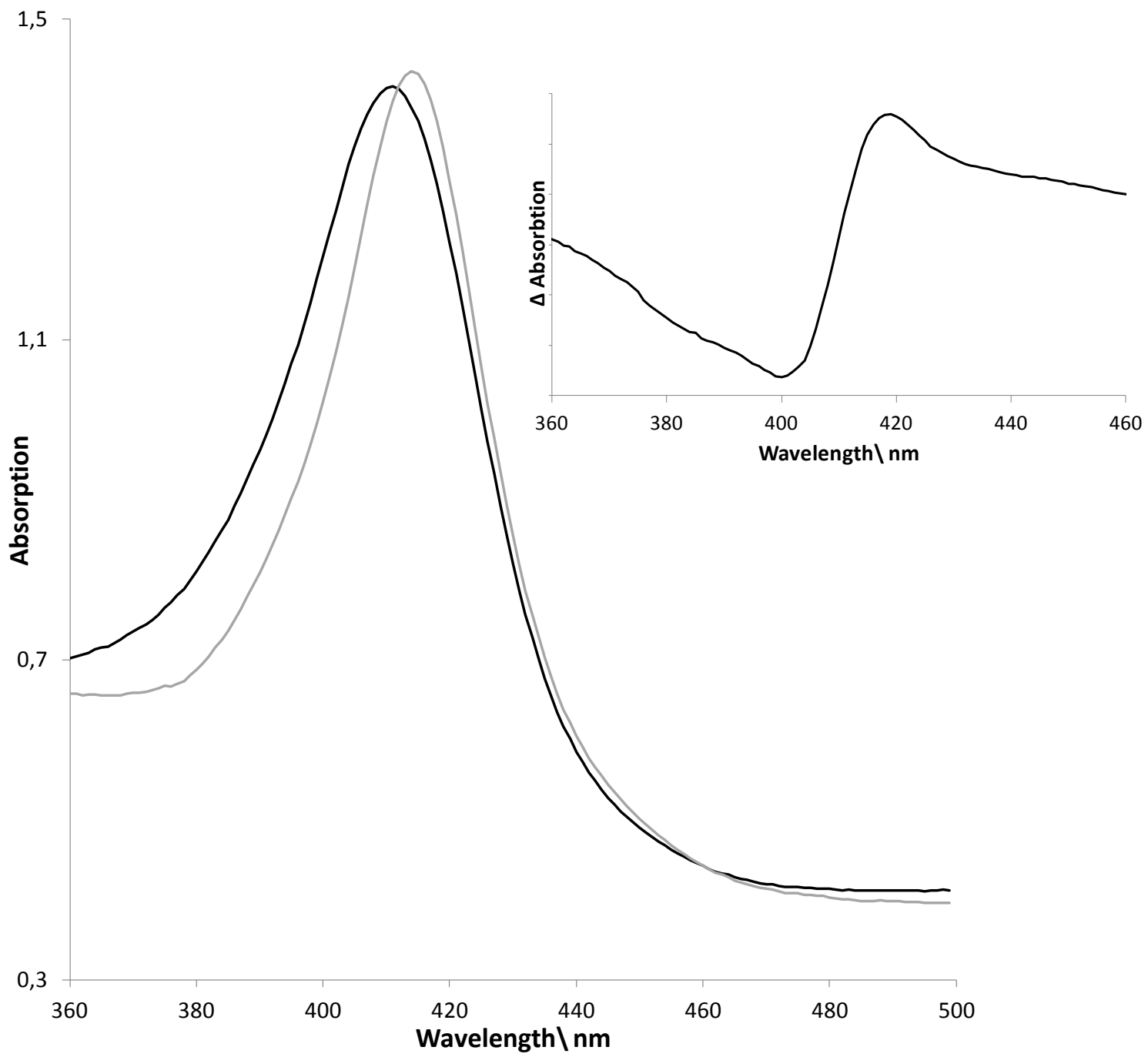

Figure 14: Vis-spectrum of $1 \mathrm{mg} / \mathrm{ml}$ PpoA in $20 \mathrm{mM}$ HEPES, pH 7.4 (black) and the same enzyme after addition of imidazole to a final concentration of $100 \mu \mathrm{M}$ (grey). The red-shift of the soret-peak, indicated by the peak at $418 \mathrm{~nm}$ and the minimum at $402 \mathrm{~nm}$ in the difference spectrum (Inset), can be interpreted by the formation of a six-coordinated nitrogen-based heme-complex and the peak-trough distance in the difference-spectrum can be used to quantify ligand-binding to the heme (Yeh et al., 2005).

\subsection{Template-based structure prediction}

Since the vast majority of proteins will not crystallize at all (Kim et al., 2008), the structure of PpoA was additionally modeled in silico. Especially proteins that have homologue protein structures already solved and deposited in the protein database (PDB; http://www.rcsb.org/pdb/home/home.do) are suitable targets for reliable template-based structure prediction. Hence, the domains of PpoA, which structurally and functional resemble characterized oxylipin synthesizing enzymes, were modeled independently from each other. 


\subsubsection{Predicted structure of PpoA's DOX-domain and identification of Residues PUTATIVELY INVOLVED IN DIOXYGENATION}

The DOX-domain of PpoA from A. nidulans was defined by to range from Met1 to His620. The thus derived sequence-string was submitted to the I-Tasser web server (zhanglab.ccmb.med.umich.edu/I-TASSER/) and modeling was initiated without assignment of templates. In a first step the algorithm identifies best aligning sequences in the PDB to optimally guide the process of fold prediction. In case of the DOX-domain, the threading process relied on PDB-entries belonging to the myeloperoxidase-family, namely: mPGHS-2 (PDB-ID: 1CVU; m Mus musculus); OPGHS-1 (PDB-ID: 1EQG; o, Ovis aries); lactoperoxidase (PDB-ID: 2GJ1) and myeloperoxidase (PDB-ID: $1 \mathrm{MHL}$ ). The resulting model of the apodomain was loaded into the molecular modelling program Chimera 1.5.3 and superimposed with both PGHS isoforms, enzymes known to exhibit a similar sequence and resembling the function of the PpoA DOXdomain (Table 1). Not surprisingly, the functional similarity was reflected on a structural level and both PGHS isoforms superimposed well with the modelled domain. As already indicated by sequence alignments, the agreement was slightly better with isoform 2 as compared to PGHS-1. Hence, a solved crystal-structure of this enzyme (PDB-ID: 3HS5) was superimposed to the predicted apodomain in order to identify presumed cofactor and substrate binding sites as well as amino acid side chains putatively responsible for reactivity (Figure $15 \mathrm{~A}$ ). The thus obtained structure with substrate and cofactor was cleaned within the Amber force field, implemented in the Chimera package.

\begin{tabular}{cccc}
\hline Homologue Enzyme & $\begin{array}{c}\text { Structural } \\
\text { superposition }\end{array}$ & Amino acid identity & Amino acid similarity \\
\hline $\begin{array}{c}\text { oPGHS-1 } \\
\text { PDB-ID: } 3 \text { N8V }\end{array}$ & 1.0 Å over $311 \mathrm{C} \alpha$ & $20.6 \%$ & $31.7 \%$ \\
\hline $\begin{array}{c}\text { mPGHS-2 } \\
\text { PDB-ID: } 3 \mathrm{HS} 5\end{array}$ & 0.9 Å over $309 \mathrm{C} \alpha$ & $16.5 \%$ & $26.7 \%$ \\
\hline
\end{tabular}

Table 1: Comparison of the modeled DOX-domain of PpoA with homologue enzyme structures previously solved. Despite sharing only an amino acid identity of $15 \%$ and an amino acid similarity of $22 \%$, oPGHS1 and mPGHS2 exhibit a highly conserved fold (Michael Garavito et al., 2002) (RMSD $=0.8 \AA$ over $533 \mathrm{C} \alpha$ ), indicating the conserved tertiary structure of heme-dioxygenases. PGHS-X, prostaglandin $\mathrm{H}_{2}$ synthase isoform $x ; m$, Mus musculus; $o$, Ovis aries

In the primary structure of the DOX-domain a highly conserved sequence motif was identified by analysis of 82 Ppo-like sequences placed in Uniprot: RxxLSDAVxLVRGDRxxT. Even at the xpositions only limited variability was observed. Despite the general homology to PGHSs and $\alpha-$ DOXs, this sequence-motif is Ppo-specific and absent in those myeloperoxidases. Strikingly, searching the Uniprot-database with this short sequence string and the default threshold (10) 
will identify solely (putative) PpoA-homologues pointing out its family specific character (BLASTretrieval from May 2012). Within the modelled structure this motif forms an $\alpha$-helix that spans over the putative substrate binding channel. Although the primary sequence is not conserved in PGHSs, the same secondary structural element can be identified in their atomic structures.

Within the modelled dioxygenase domain of PpoA, the heme is placed between the two histidines (His202, His377) that already have been identified by sequence alignments as distal and proximal heme ligands in 7,8-linoleate diol synthase (Garscha and Ernst H Oliw, 2008) (Figure $15 \mathrm{C}$ ). Resembling the substrate channel of PGHS, the fatty acid substrate might be bound in a channel lined by hydrophobic amino acids, which could contribute by hydrophobic interactions to binding of the substrate (Figure $15 \mathrm{~B}$ and $17 \mathrm{~A}$ ). For PGHS, it was shown that substrate affinity is additionally mediated by ionic interaction of the fatty acid's carboxyl group with the guanidinium group of arginine 120 (numbering of oPGHS1) (Koszelak-Rosenblum et al., 2008). In the modeled structure, no basic amino acid structurally aligns with this position, but the charge of arginine 336 is in equal distance to the substrate's carboxylate (5 A; Figure $15 \mathrm{C}$ and 17 A). Tyrosine 374 , the homologue position to mPGHS-2's catalytic active Tyr385, was already shown to be involved in hydrogen-abstraction from carbon $\mathrm{C}_{8}$ (Garscha and Ernst $\mathrm{H}$ Oliw, 2008; Brodhun et al., 2009; Fielding et al., 2011) and is placed in a reasonable distance to this carbon. Notably, the distance between this tyrosine and the hydrogen to be abstracted was modeled to be almost twice as large as the corresponding distance between tyrosine 385 and $C_{13}$ in mPGHS-2. In contrast, tyrosine 327 of the PpoA DOX-domain is located in a distance to $C_{8}$ that is only half the catalytic distance found in $\mathrm{mPGHS}-2$ and thus might rather resemble a catalytic competent interaction. Still it should be mentioned that the substrate position between these two tyrosine residues is rather free and also different substrate topographies with tyrosine 374 being closer to $C_{8}$ are feasible. To shed light on the role of the two newly identified amino acid determinants, an Arg336Met- and a Tyr327Phe-variant were constructed in addition to the already established Tyr374Phe-variant (Brodhun et al., 2009). 


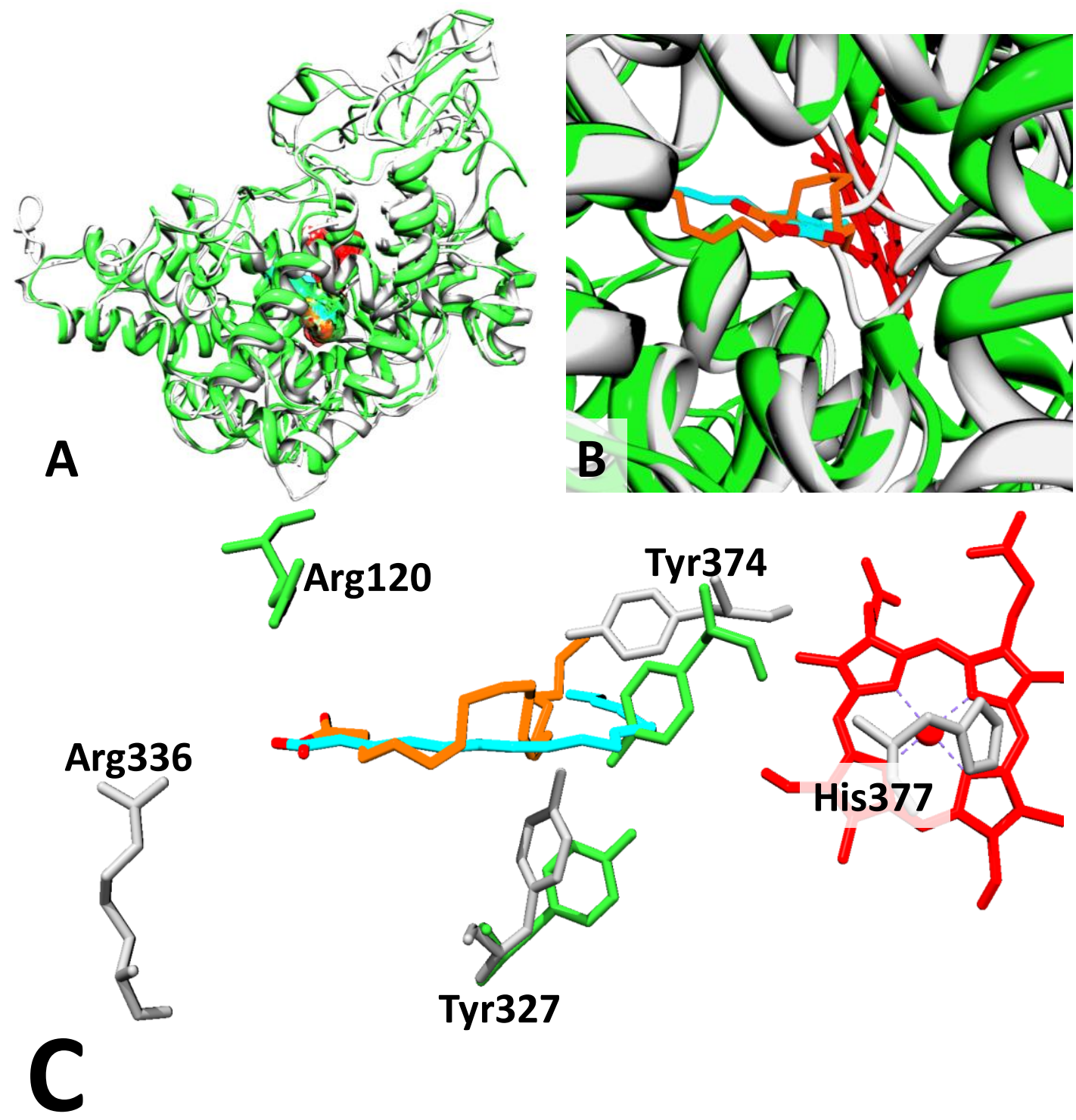

Figure 15: Predicted structure of PpoA's DOX-domain. Shown is the predicted model (white) superimposed with mPGHS2 (green; PDB-ID: 3HS5) serving as relevant homologue enzyme. A Overall structure of the PpoA model superimposed to the experimental structure of mPGHS2. The RMSD between both structures is $0.9 \AA$ over $309 \mathrm{C} \alpha$. B Zoom into the active site. Linoleic acid (rendered in cyan) was placed within the predicted PpoA structure in a similar position and conformation as the substrate (arachidonic acid, orange) is bound in MPGHS2. C Determinants involved in substrate conversion catalyzed by MPGHS-2 and amino acids, proposed to play an analogous role in PpoA, are shown. Arg120 of mPGHS-2 was identified to contribute to arachidonic acid binding by ionic interaction with the carboxylate group. Although in a different position, Arg336 in PpoA is in a reasonable distance to promote substrate affinity by the same mechanism. PpoA's Tyr374 aligns with mPGHS2's Tyr385 and the radical intermediate of this residue is most likely also involved in hydrogen abstraction from the fatty acid substrate. Tyr327 (PpoA) might either serve as an alternative radical site or assist in orienting Tyr374 by formation of a hydrogen-bonding network. At least it aligns with mPGHS2's Tyr348 that was proposed to fulfill the latter role. Also the heme coordinating histidines are structurally conserved in both enzymes. Exemplary shown is PpoA's proximal histidine (HIS377). 


\subsubsection{Predicted structure of PpoA's P450-domain and IDENTIFICATION OF Residues PUTATIVELY INVOLVED IN HYDROPEROXY FATTY ACID REARRANGEMENT}

For modeling of PpoA's hydroperoxide isomerase-domain, the sequence-string from His620 to Phe1081 was submitted to the I-Tasser web interface. Again, initially no templates were specified and thus the threading process unbiasedly searched the PDB. The main templates used to generate the final structure were: $\mathrm{CYP}_{400} \mathrm{CalO}_{2}$ (PDB-ID: 3BUJ); CYP450 46A1 (PDB-ID: 2Q9F); CYP450 105P1 (PDB-ID: 3E5J); CYP450 MoxA (PDB-ID: 2Z36); CYP450 cin (PDB-ID: 1T2B); CYP450 cam (PDB-ID: 1YRC) and CYP450 154C1 (PDB-ID: 1GWI). As expected all templates belong to the cytochrome P450 enzyme family, but surprisingly none of the utilized templates was a class IIIcytochrome P450. To assess the potential differences between the modelled structure and typical cytochrome P450s, the model was superimposed with a number of class III P450s and P450cam, the prototype P450 from Pseudomonas putida (Table 2).

\begin{tabular}{cccc}
\hline Homologue Enzyme & $\begin{array}{c}\text { RMSD of Structural } \\
\text { superposition }\end{array}$ & Amino acid identity & Amino acid similarity \\
\hline $\begin{array}{c}\text { AtAOS } \\
\text { PDB: } 2 \text { RCH }\end{array}$ & 1.3 Å over $123 \mathrm{C} \alpha$ & $11.5 \%$ & $21.5 \%$ \\
\hline $\begin{array}{c}\text { PaAOS } \\
\text { PDB: } 3 \mathrm{DBM}\end{array}$ & $1.2 \AA$ over $113 \mathrm{C} \alpha$ & $14.5 \%$ & $25.3 \%$ \\
\hline $\begin{array}{c}\text { hCyp8a } \\
\text { PDB: } 2 \mathrm{IAG}\end{array}$ & $1.3 \AA$ A over $70 \mathrm{C} \alpha$ & $16.6 \%$ & $26.8 \%$ \\
\hline $\begin{array}{c}\text { P450cam } \\
\text { PDB: } 2 Z W T\end{array}$ & $1.3 \AA$ over $91 \mathrm{C \alpha}$ & $21.7 \%$ & $30 \%$ \\
\hline
\end{tabular}

Table 2: Comparison of the modeled P450-domain of PpoA with homologue enzyme structures previously solved. AOS, allene oxide synthase; At, Arabidopsis thaliana; $P a$, Parthenium argentatum; hCyp8a human prostacyclin synthase; P450cam, Pseudomonas putida camphor monooxygenase

The model superimposes with all structures to a similar RMSD, which points out the fact that, despite sometimes quite diverse primary structures, the fold of cytochrome P450s is highly conserved (Sirim et al., 2010). Due to functional similarities and good structural superposition, the AOS structures were chosen to guide the process of cofactor and substrate placement as well as identification of amino acids, putatively involved in catalysis.

Despite the reasonable structural agreement between the modelled domain and solved structures of homologue enzymes, the later on experimentally verified role of three amino acids in substrate-positioning, regioselectivity of substrate hydroxylation and peroxide-cleavage led to the need to refine the model. Therefore, the I-helix was readjusted and the F-helix was repositioned from the entrance of the substrate access channel to a position directly facing the heme on the distal site. This latter displacement was necessary to bring two phenylalanines 
(Phe795 and Phe799), proposed to be involved in substrate positioning, in a position in which the distance between substrate and heme is catalytic relevant. This reorientation might reflect a dynamic process naturally occurring upon substrate binding to cytochrome P450s (Sirim et al., 2010). The thus perturbed structure was specified as template for a new submission of this domain's primary sequence to the I-Tasser-server. The algorithm's inherent force field cleaned the perturbed structure and the resulting apodomain showed an overall-fold and quality parameters (Q-Mean; swissmodel.expasy.org/qmean) comparable to the initial model. In fact the RMSD between this "closed, substrate-bound" conformation of PpoA's hydroperoxide isomerase domain and the previously obtained "opened, substrate-free" form was $0.98 \AA$ over $345 \mathrm{C} \alpha$. Furthermore, the "closed"-state structurally aligned to the same extent with the given set of prototype P450s (Table 3).

\begin{tabular}{ccc}
\hline Homologue Enzyme & $\begin{array}{c}\text { RMSD of Structural } \\
\text { superposition before } \\
\text { manual refinement }\end{array}$ & $\begin{array}{c}\text { RMSD of Structural } \\
\text { superposition after manual } \\
\text { refinement }\end{array}$ \\
\hline $\begin{array}{c}\text { AtAOS } \\
\text { PDB: } 2 \text { RCH }\end{array}$ & $1.3 \AA$ over $123 \mathrm{C} \alpha$ & $1.1 \AA$ over $102 \mathrm{C} \alpha$ \\
\hline $\begin{array}{c}\text { PaAOS } \\
\text { PDB: } 3 \mathrm{DBM}\end{array}$ & $1.2 \AA$ over $113 \mathrm{C} \alpha$ & $1.0 \AA$ over $94 \mathrm{C} \alpha$ \\
\hline $\begin{array}{c}\text { hCyp8a } \\
\text { PDB: } 2 \text { IAG }\end{array}$ & $1.3 \AA$ over $70 \mathrm{C} \alpha$ & $1.3 \AA$ over $96 \mathrm{C} \alpha$ \\
\hline $\begin{array}{c}\text { P450cam } \\
\text { PDB: } 2 Z W T\end{array}$ & $1.3 \AA$ over $91 \mathrm{C} \alpha$ & $1.3 \AA$ over $106 \mathrm{C} \alpha$ \\
\hline
\end{tabular}

Table 3: Comparison of the refined model of PpoA's P450-domain with homologue enzyme structures previously solved. AOS, allene oxide synthase; At, Arabidopsis thaliana; $P a$, Parthenium argentatum; hCyp8a human prostacyclin synthase; P450cam, Pseudomonas putida camphor monooxygenase

In contrast to the initial, "opened" model, several later on experimentally verified amino acid determinants for certain aspects of reactivity are placed in a meaningful spatial arrangement within the "closed" form (Figure 16). For this model AtAOS was chosen to define an appropriate substrate position, since in this structure the substrate is placed in a position, relative to heme, that is more likely to be catalytic competent as compared to the solved PaAOS structure. Figure $16 \mathrm{C}$ highlights some residues that may play a role in hydroperoxide rearrangement. First, substrate binding and -positioning might be mediated by $\pi$-stacking between the substrate's $\Delta 9$ double bond and the aromatic phenyl ring of Phe795. Although Phe799 might interact in a similar way with the $\Delta 12$-double bond of the substrate, the larger distance between its phenyl ring and the double bond suggests rather another role for this amino acid and it might be involved in constraining the substrate position by the bulkiness of its side-chain. As proposed for the DOX-domain, additional contributions to substrate binding might arise from ionic interactions between the substrate's carboxylate and a positively charged amino acid side chain. 
However, the substrate is proposed to enter the active-site of the P450-domain with its carboxyl-tail first (Figure $16 \mathrm{~B}$ and $17 \mathrm{~B}$ ) and thus a deprotonated substrate may be repulsed by the mainly uncharged active site. Moreover, no basic amino acid that could coordinate the carboxylate was found in reasonable distance within the modeled active site. Finally, Asn887 located in the I-helix is placed in a proper position to form a hydrogen bonding network between its amide-function, the heme-iron and the substrate's peroxide. This hydrogen-bonding network might be crucial for substrate-binding and heterolytic cleavage of the peroxides O-O-bond. To probe the role of the aforementioned putative determinants, respective enzyme-variants (Phe795Leu; Phe799Leu; Phe799Met; Phe799Tyr; Phe799Trp and Asn887Val) were constructed by site-directed mutagenesis.

Figure 16 (next page): Predicted structure of PpoA's P450-domain (white) superimposed with AtAOS (blue; PDB-ID: 2RCH) serving as relevant homologue enzyme. A Overall structure of the PpoA model superimposed to the experimental structure of AtAOS. The RMSD between both structures is $1.3 \AA$ over 123 C $\alpha$. B Zoom into the active site. 8-HPODE (rendered in cyan) was placed within the predicted PpoA structure in a similar position and conformation as the substrate-analogue (13-HODE, orange) is bound in AtAOS. C Determinants involved in substrate conversion catalyzed by AtAOS and amino acids, proposed to play an analogous role in PpoA are shown. Asn321 of AtAOS was identified to contribute to catalytic turnover by formation of a hydrogen-bonding network and thus facilitating the cleavage of the peroxide's $O-O$ bond (D.-S. Lee et al., 2008). Although in a slightly different position, Asn887 in PpoA is in a reasonable distance within the I-helix to putatively promote substrate conversion by a similar mechanism. As expected, the cysteines coordinating the heme-iron as fifth ligand are conserved in both structures (Cys1006 in PpoA and Cys471 in AtAOS). Also a positively charged residue stabilizing the heme by ionic interaction with one of the protoporphyrin's propionates is conserved in both structures (His1004 in PpoA and Lys469 in AtAOS). Additional to these residues, a phenylalanine-pair (Phe795 and Phe799) seems to be of relevance for proper substrate binding and placement in PpoA. 


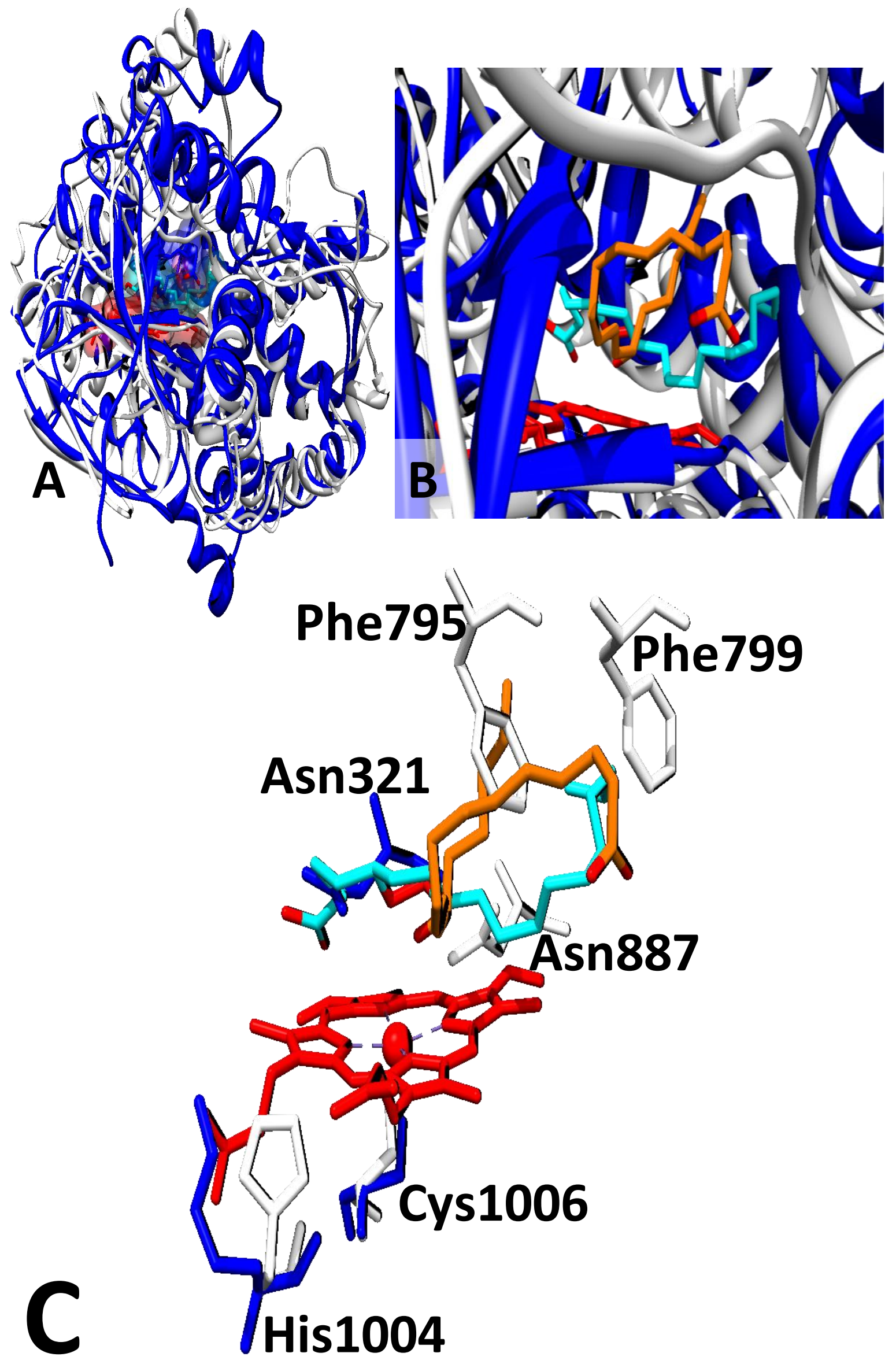




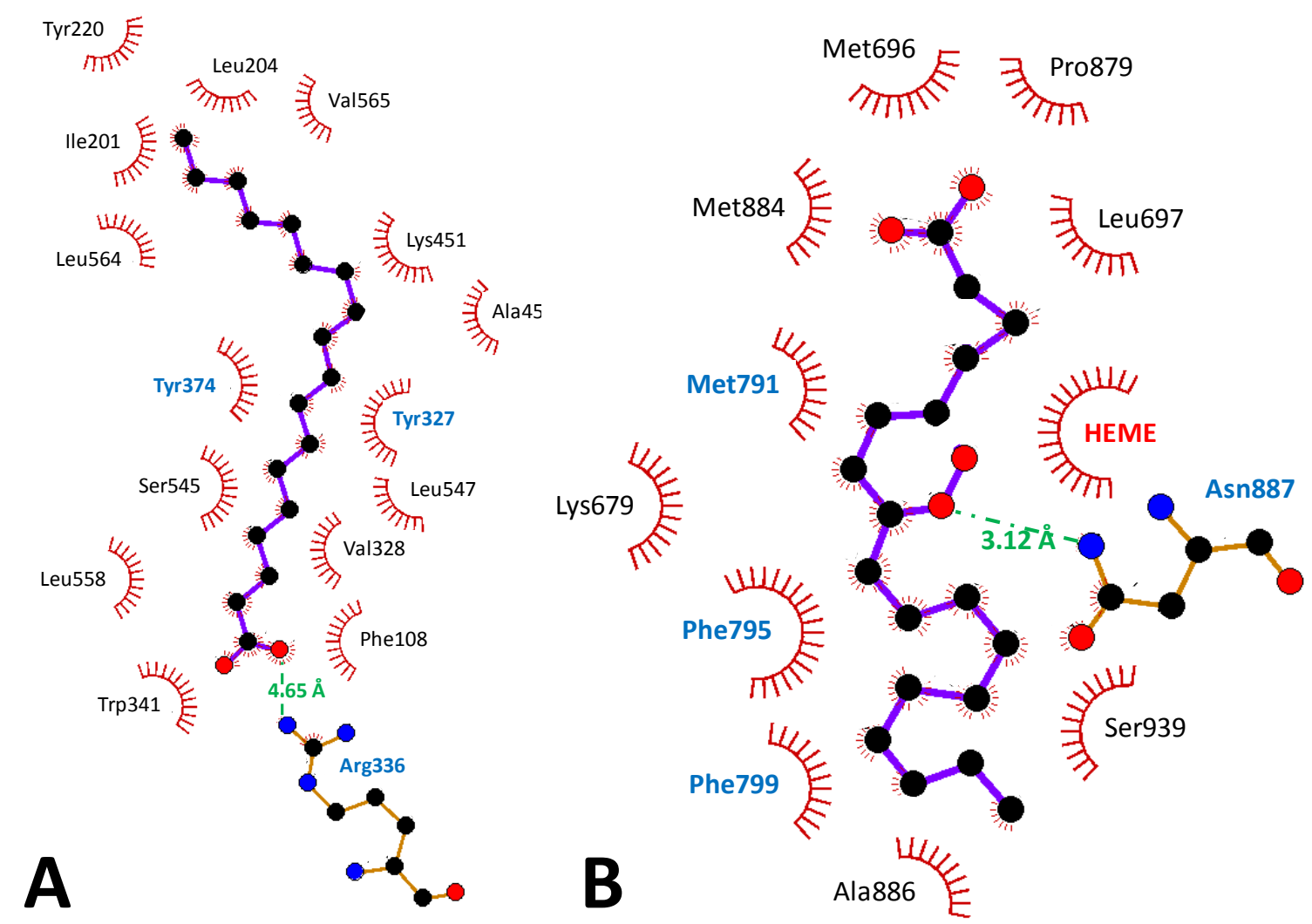

Figure 17: 2D-representation of amino acids forming the active site of PpoA's DOX-domain (A) and P450domain (B), respectively. The substrate is shown in purple, carbons are colored black, nitrogen is blue and oxygen is depicted in red. Amino acids forming hydrophobic contacts with the fatty acid substrate are visualized as semicircles and amino acids involved in hydrogen bonds with the substrate are depicted in ball-stick representation. The corresponding hydrogen bond is shown. In contrast to the DOX-domain, the substrate seems to enter the P450-active site with its carboxyl-end first and exhibits a direct interaction with the heme co-factor. Amino acids investigated in this study are highlighted in blue. The representations were calculated with LigPlot ${ }^{+}$(Laskowski and Swindells, 2011).

\subsection{Validation of proposed amino acid determinants by biochemical characterization of respective variants}

\subsubsection{Substrate binding to PpoA's DOX-Domain may be GOVERnED by IONIC INTERACTION WITH ARG336}

From the modeled structure it was deduced that an important contribution to substrate binding might arise from ionic interactions between the carboxylate and the positively charged guanidinium group of Arg336. Nevertheless, one should keep in mind that the hydrophobic nature of the substrate suggests that main contributions to substrate affinity might arise from the fatty acid tail interacting with hydrophobic residues forming the active site (Figure $17 \mathrm{~A}$ ). To estimate the importance of the ionic interaction between the carboxylate and Arg336 for 
substrate affinity, an uncharged enzyme variant (Arg336Met) was constructed and its kinetics was compared to that of wild type enzyme (Figure 18). The shown kinetic traces were obtained by measuring the depletion of dissolved oxygen upon mixing a varying concentration of linoleic acid with $100 \mathrm{nM}$ of the respective enzyme in $20 \mathrm{mM}$ HEPES buffer, $\mathrm{pH}$ 7.4. For evaluation, the maximal slope of this depletion was determined and, assuming a stoichiometric reaction of one molecule dioxygen with one molecule fatty acid, converted to a substrate conversion rate. Interestingly, the obtained data for the Arg336Met variant suggest a sigmoidal behavior rather than a hyperbolic one as it is observed for wild-type enzyme kinetics and thus suggests a different reaction mechanism. Nevertheless, the data are not significant enough to base a detailed assessment of different kinetic models on them and a putative change of enzyme cooperativity caused by the conducted mutation is not explained straightforwardly. Therefore, both datasets were fitted with a hyperbolic equation and the obtained parameters are: $\mathrm{k}_{\mathrm{m}}=15.3$ $\mu \mathrm{M}$ and $\mathrm{k}_{\mathrm{cat}}=399 \mathrm{~min}^{-1}$ for wild type enzyme and $\mathrm{k}_{\mathrm{m}}=49.5 \mu \mathrm{M}$ and $\mathrm{k}_{\mathrm{cat}}=231 \mathrm{~min}^{-1}$ for the Arg336Met-variant of PpoA. Although the fit with a sigmoidal kinetics $\left(v=\frac{v \max *[L A]^{n}}{k m^{n}+[L A]^{n}}\right.$; (Witherow and Houston, 1999)) led to a much better description of the dataset measured for the Arg336Met-variant (least square sum improved from 66.4 to 8.1), the derived kinetic parameters differ by the same magnitude between wild type and the variant and the better fit was caused merely by a changed Hill coefficient $\left(k_{m, w t}=13.7 \mu \mathrm{M} ; k_{c a t, w t}=375.4 \min ^{-1} ; n_{w t}=1.2\right.$ and $\left.k_{m, R 336 M}=30.4 \mu \mathrm{M} ; k_{c a t, R 336 M}=167.1 \mathrm{~min}^{-1} ; n_{R 336 M}=5.0\right)$. One should also keep in mind that the values obtained for $k_{c a t}$ and $k_{m}$ have to be seen as an approximation of the true value, since micelle-formation of the substrate molecules might lead to an underestimation of the $k_{m}$-values, especially for the Arg336Met-variant.

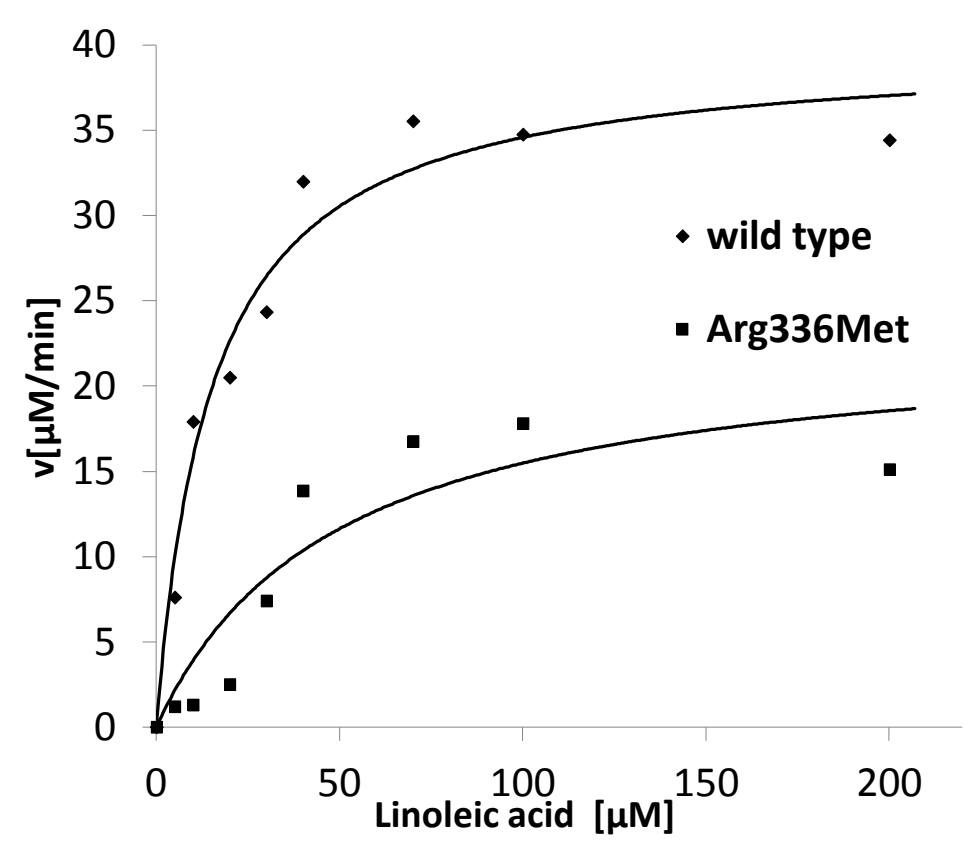

Figure 18: Kinetics of linoleic acid conversion by PpoA_wild type enzyme and PpoA_Arg336Met. $100 \mathrm{nM}$ of the respective enzyme in $20 \mathrm{mM}$ HEPESbuffer, $\mathrm{pH}$ 7.4, were converted with varying substrate concentrations. The conversion rate was determined from the consumption rate of dissolved dioxygen. The measured data were fit to obey Michaelis-Menten kinetics and the calculated parameters are: $k_{m, w t}=$ $15.3 \mu \mathrm{M} ; k_{\text {cat }, w t}=399 \mathrm{~min}^{-1} ; k_{m, R 336 \mathrm{M}}=$ $49.5 \mu \mathrm{M} ; k_{\text {cat, } R 336 \mathrm{M}}=231 \mathrm{~min}^{-1}$. Shown is one of two independently determined kinetics. 


\subsubsection{TYR327: A SECOND TYROSINE INVOLVED IN HYDROGEN ABSTRACTION FROM THE FATTY ACID SUBSTRATE'S $\mathrm{C}_{8}$ ?}

Tyrosine 374 of PpoA was proposed to be involved in a radical-mechanism finally leading to abstraction of hydrogen from the substrate's $C_{8}$ (Brodhun et al., 2009; Fielding et al., 2011). The thus formed substrate centered radical reacts with molecular oxygen and yields, via a peroxyl intermediate, the product of PpoA's DOX-domain: 8-hyproperoxy fatty acid. Within the predicted structure of PpoA's DOX-domain, the tyrosine proposed to initially abstract hydrogen form the fatty acid's $C_{8}$ is only one of two tyrosines in roughly equidistant positions to the substrate's $C_{8}$. There are several roles that the other tyrosine (Tyr327) could play within the catalytic mechanism. Among those are i) a putative alternative radical-site; ii) hydrogen-bonding to Tyr374 and orienting this residue in a catalytic competent conformation suitable to interact with the hydrogen of the substrate's $C_{8}$ and iii) $\pi$-stacking between the phenoxyl-ring of Tyr327 and the $\Delta 9$-double bond of the substrate in order to place the substrate's $C_{8}$ in an adequate distance to Tyr374. To establish whether Tyr327 indeed can be attributed to one of those roles, again an oxygen depletion kinetics was obtained (Figure 19). The data show that Tyr327 is crucially involved in catalytic turnover $\left(k_{m, w t}=15.3 \mu \mathrm{M} ; k_{c a t, w t}=399 \min ^{-1} ; k_{m, y 327 F}=44.9 \mu \mathrm{M}\right.$; $k_{\text {cat, }, 327 \mathrm{~F}}=87 \mathrm{~min}^{-1}$ ). Again the shape of the kinetics is altered, which suggests a transition towards another oxidation mechanism caused by the single point mutation. A sigmoidal description of the measured kinetics results once more in a significantly improved fit (least square sum improved from 8.0 to 1.4), but, with exception of the Hill coefficient, the derived kinetic parameters for wild type and the variant differ by the same magnitude as those obtained for the Michaelis-Menten mechanism ( $k_{m, w t}=13.7 \mu \mathrm{M} ; \mathrm{k}_{\text {cat,wt }}=375.4 \mathrm{~min}^{-1} ; \mathrm{n}_{\mathrm{wt}}=1.2$ and $\mathrm{k}_{\mathrm{m}, \mathrm{Y} 327 \mathrm{~F}}=27.4$ $\mu \mathrm{M} ; \mathrm{k}_{\mathrm{cat}, \mathrm{Y327 \textrm {F }}}=63.3 \mathrm{~min}^{-1} ; n_{\mathrm{Y} 327 \mathrm{~F}}=3.8$. Here it should be emphasized that, although this experiment pointed out the pivotal role of Tyr327 in substrate turn-over, no clarification on the nature of its mechanism is achieved.

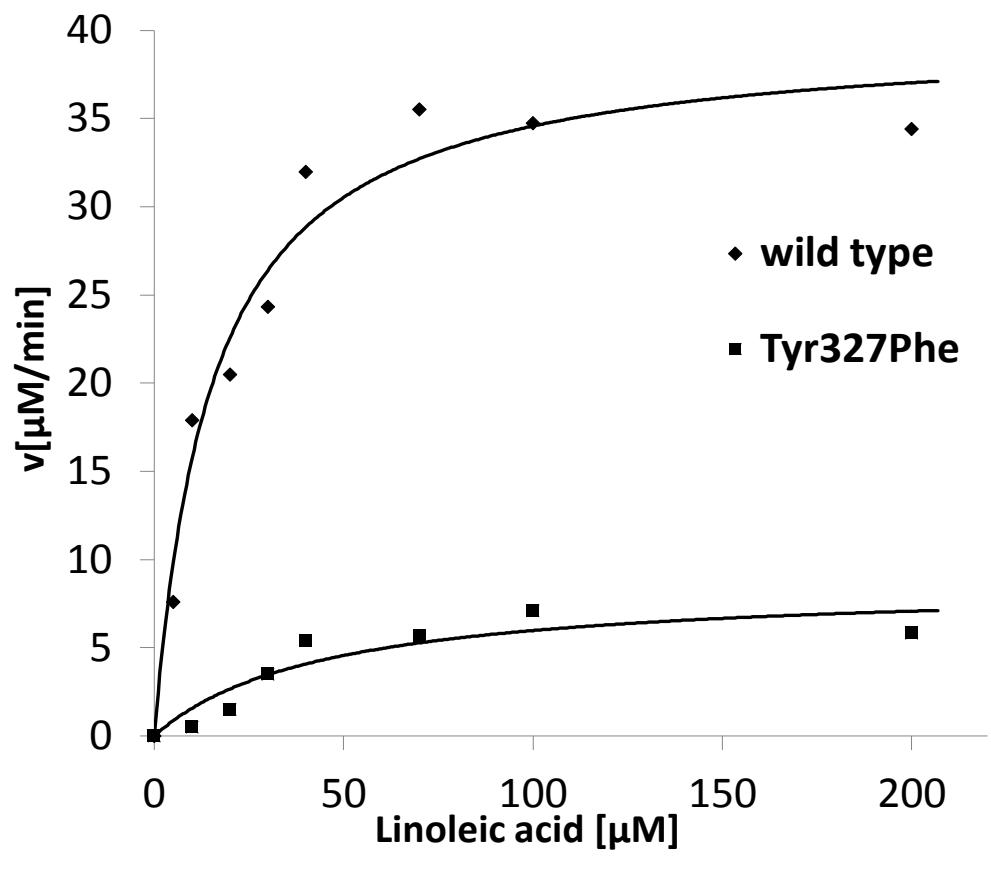

Figure 19: Kinetics of linoleic acid conversion by PpoA_wild type enzyme and PpoA_Tyr327Phe. $100 \mathrm{nM}$ of the respective enzyme in $20 \mathrm{mM}$ HEPESbuffer, $\mathrm{pH}$ 7.4, were converted with varying substrate concentrations. The conversion rate was determined from the consumption rate of dissolved dioxygen. The raw data were fit to a Michaelis-Menten kinetics and the calculated parameters are: $\mathrm{k}_{\mathrm{m}, \mathrm{wt}}=15.3$ $\mu \mathrm{M} ; \mathrm{k}_{\text {cat }, \mathrm{wt}}=399 \mathrm{~min}^{-1} ; \mathrm{k}_{\mathrm{m}, \mathrm{Y} 327 \mathrm{~F}}=44.9$ $\mu \mathrm{M} ; \mathrm{k}_{\mathrm{cat}, \mathrm{Y} 327 \mathrm{~F}}=87 \mathrm{~min}^{-1}$. Shown is one of two independently determined kinetics. 
To estimate whether Tyr327 influences the conformation of the tyrosine radical in the activated enzyme, the tyrosyl-radical of the Tyr327Phe-variant was trapped and subsequently analyzed by EPR-spectroscopy at different frequencies (Experiment conducted and evaluated by Dr. Alistair Fielding in the working group of Prof. Dr. Marina Bennati; MPI for biophysical Chemistry, Goettingen). The measured g-values of the formed radical are consistent with a tyrosyl species, but the observed ß-proton splitting is narrower than in the wild type, which could be interpreted by a different dihedral-angle between the radical's phenoxyl ring and the ß-protons. Additionally, simulating the measured radical by one tyrosyl was not possible, which suggests the presence of different tyrosyl rotamers. On the one hand, these results might be interpreted in a way substantiating the hypothesis of Tyr327 stabilizing Tyr374 in a conformation suitable for hydrogen abstraction from $\mathrm{C}_{8}$ and thus disrupting of the respective hydrogen bond would cause the Tyr374 to adopt another conformation. On the other hand the result of this measurement is also consistent with the idea that the catalytic competent radical is formed at Tyr327 and Tyr374 is merely a transient link in an electron transfer pathway from Tyr327 to heme compound I. Hence, by preventing formation of the radical at position 327 in PpoA's Tyr327Phe-variant the radical could be trapped at Tyr374, a tyrosine putatively exhibiting a different conformation than the one measured in wild type. Moreover, an additional role of Tyr327's phenoxyl ring in positioning the substrate's $\Delta 9$ double bond by $\pi$-stacking is not excluded by these data. Therefore two supplementary variants were constructed: One variant with an aliphatic, hydrophilic residue (Tyr327GIn) and one variant with an aliphatic, hydrophobic residue (Tyr327Leu). While the Tyr327GIn-variant was not reacting with linoleic acid, the Tyr327Leuvariant, which is supposed to have neither the ability to properly place the substrate nor to orient the Tyr374-radical by hydrogen bonds, exhibited low residual activity. Although the DOXdomain formed not only 8-HPODE, but also 10-HPODE, the amount of this by-product was comparable to what can be found in wild type enzyme. $75 \%$ of the hydroperoxy fatty acid produced by the Tyr327Leu-variant was 8-HPODE, a value comparable to the $60-70 \%$ reported for PpoA wild type enzyme (Brodhun et al., 2010). In a nutshell, the product pattern of this variant contradicts the hypothesis of an aromatic residue at position 327 being involved in correct placement of the substrate and therefore determining regioselectivity of fatty acid oxidation. However, Tyr327 is crucially involved in catalysis and this observation might be explained either by a hydrogen bond between this residue and the catalytic competent Tyr374 or an (alternative) tyrosyl radical formed at this position. To probe the latter hypothesis, the distances between the tyrosyl-radicals in the distinct enzyme monomers were measured by DEER and the amino acid position of the radical was assigned based on a low resolution structure of PpoA (Section 4.6.2). 


\subsubsection{Determinants of SUbStRate binding to PPoA'S hydRoperoxide-ISOMERASE- DOMAIN}

To probe whether ionic interactions between the substrate's carboxylate and a positively charged amino acid within the active site play a role in substrate binding to PpoA's cytochrome P450-domain, conversion of 8-HPODE methyl ester was pursued. This uncharged substrate analogue should be repulsed by a positive charge within the active site and thus no product conversion would be detectable, if a basic amino acid is involved in substrate binding. Interestingly, the results not only show that 8-HPODE methyl ester can be rearranged by PpoA (Figure $20 \mathrm{~A}$ ), but a competitive assay, in which equal amounts of 8-HPODE and 8-HPODE methyl ester were provided for the rearrangement reaction, revealed that the enzyme has the same conversion rate for both kinds of substrate (Figure $20 \mathrm{~B}$ ). A final experiment to prove the derived hypothesis that no charges are involved in substrate binding to PpoA's hydroperoxide isomerase domain was to conduct the aforementioned competitive assay at a higher $\mathrm{pH}$. While the previous assay was performed at $\mathrm{pH} 7.4$ (50 mM TRIS-buffer) and at this $\mathrm{pH}$ obviously both substrates were converted to an equal extent, the same experiment performed at pH $9(50 \mathrm{mM}$ TRIS-buffer) resulted in conversion of solely 8-HPODE methyl ester. The most obvious explanation for this result is repulsion of the deprotonated substrate from the enzyme's noncharged active site structure. In line with this observation, conversion of linoleic acid by PpoA yields a product pattern that crucially depends on the buffer's pH. At pH 5 and 7 only traces of hydroperoxy fatty acid accumulate, but the vast majority is rearranged to 5,8-DiHODE. In contrast, at $\mathrm{pH} 9$ the situation differs and 8-HPODE is accumulated and not rearranged by the enzyme's P450 activity (data not shown). 
Results
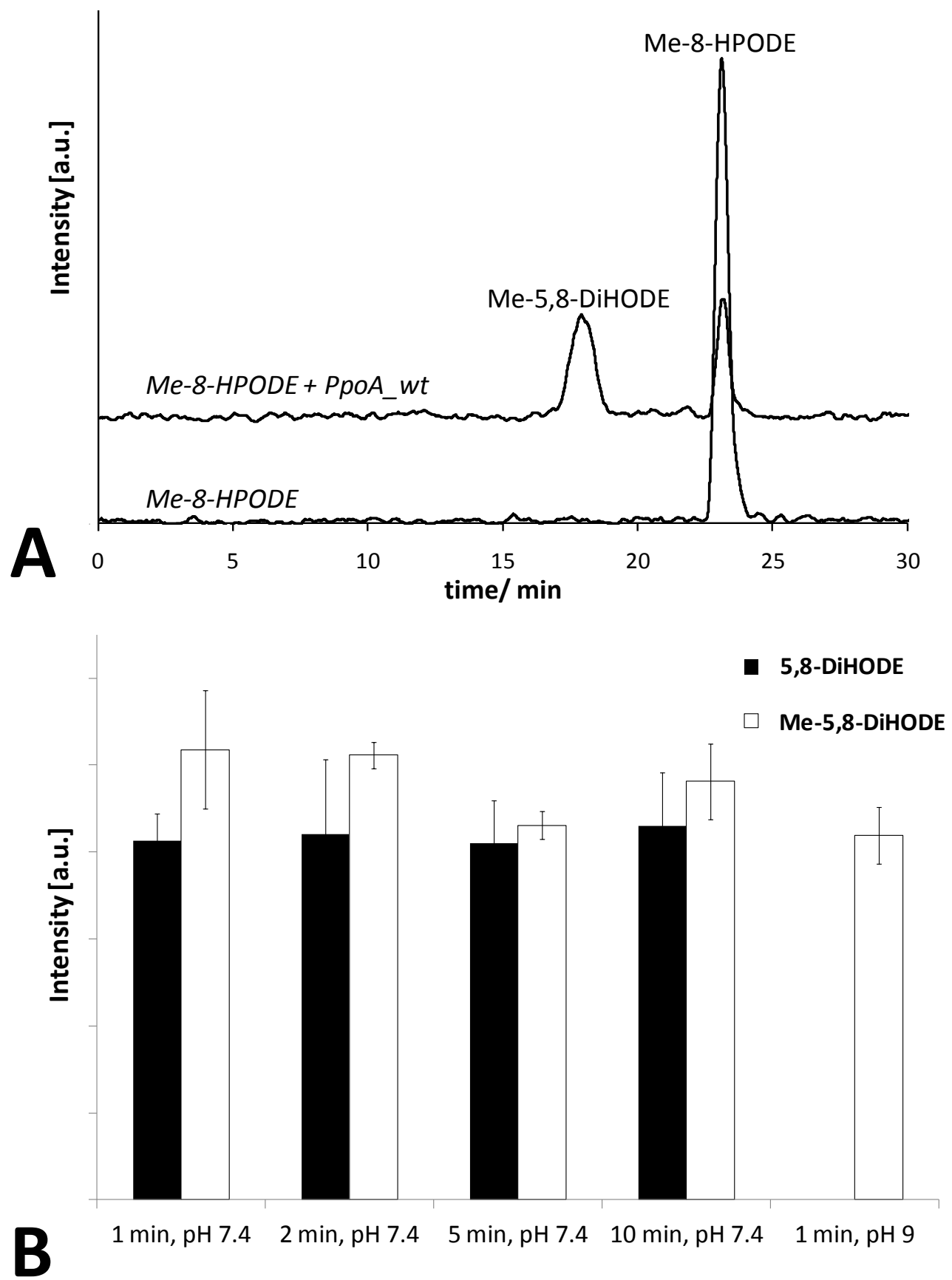

Figure 20: No ionic interactions are involved in substrate binding to PpoA's hydroperoxide isomerase domain. $\boldsymbol{A}{ }^{14} \mathrm{C}$-labeled 8-HPODE methyl ester was synthesized by conversion of ${ }^{14} \mathrm{C}$-linoleic acid with PpoA_C1006A (Brodhun et al., 2009) and subsequent methylation of the resulting ${ }^{14}$ C-8-HPODE with trimethylsilyldiazomethane. This substance (lower trace) is converted by PpoA (wild type) to 5,8-DiHODE methyl ester (upper trace). Shown are representative scintillation counter detected signals of RP-HPLC separated substances. B A competitive assay in which equal amounts of 8-HPODE methyl ester and 8HPODE were subjected to conversion by PPOA (wild type) and were extracted after different times resulted in formation of equal amounts 5,8-DiHODE and 5,8-DiHODE methyl ester at pH 7.4. At pH 9.0 only conversion of 8-HPODE methyl ester was observed. Shown are the arithmetic means of three independent conversions quantified for each time point as well as the corresponding standard deviation. 
While these results demonstrate that ionic interactions do not play a role in substrate binding and thus validate the absence of basic amino acids in the modeled active site and support the hypothesis that the substrate may enter the P450-active site with its carboxyl-end first, binding of the substrate to aromatic residues via $\pi$-stacking is still reasonable.

$\pi$-Stacking as potential constrain for proper placement of 8-HPODE in PpoA's P450-domain

Evaluating the predicted structural model of PpoA's P450-domain, one can hypothesize that Phe795 is involved in correct substrate orientation by $\pi$-stacking of its aromatic side chain with the substrate's $\Delta 9$ double bond. The initial, "opened" homology model of the P450-domain furthermore suggested that another highly conserved phenylalanine (Phe799) could interact with the $\Delta 12$ double bond of polyenoic fatty acid derivatives (Figure 21). Both phenylalanines are located in the region in between the F/G-helices, which is described to typically harbor the substrate recognition sites (SRS) 2 and 3 (Sirim et al., 2010).

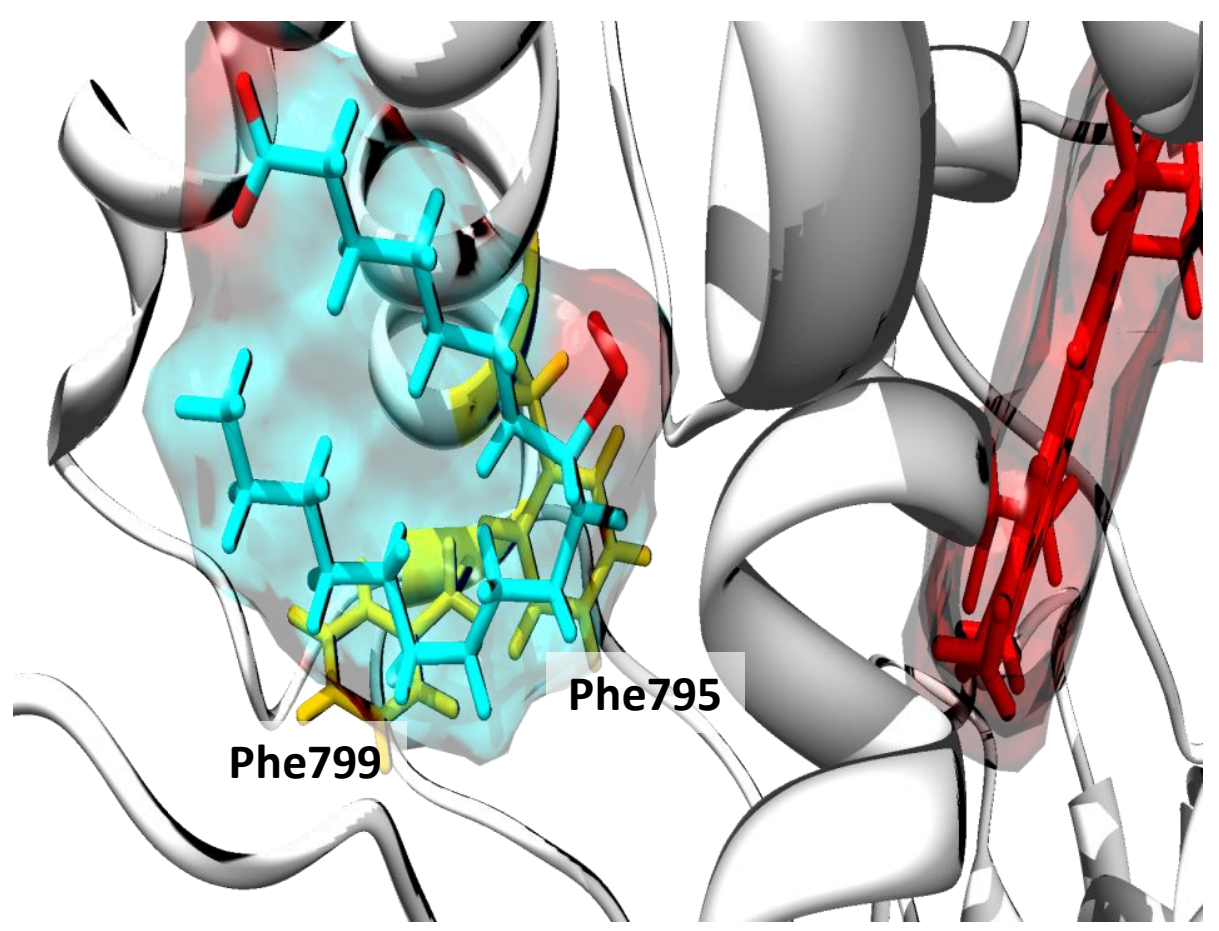

Figure 21: Aromatic residues, putatively involved in $\pi$-stacking to place the polyenoic substrate in the right position. In this substrate orientation, Phe795 is in proximity to the fatty acids $\Delta 9$ double bond and Phe 799 might be placed to stabilize the position of the $\Delta 12$ double bond. It should be mentioned that the distance between the peroxide and the heme is likely to large for this conformation to be a productive state and thus a conformational change and closure of the enzyme-domain upon substrate binding seems to be likely. 
Results

To elucidate whether the aromaticity of these phenylalanines indeed might play a role in substrate positioning, the respective leucine-variants and a double mutant were created and the product pattern observed upon conversion of oleic and linoleic acid was determined. The results of oleic acid conversion by the Phe795Leu-variant illustrate that Phe795 plays a role in determining the regioselectivity of the reaction (Figure 22). In the product-pattern obtained from conversion with the variant, the amount of DiHOME with changed regiochemistry is increased ten-fold from $2.4 \%$ of the total amount of products to $24 \%$ as compared to the wild type enzyme.

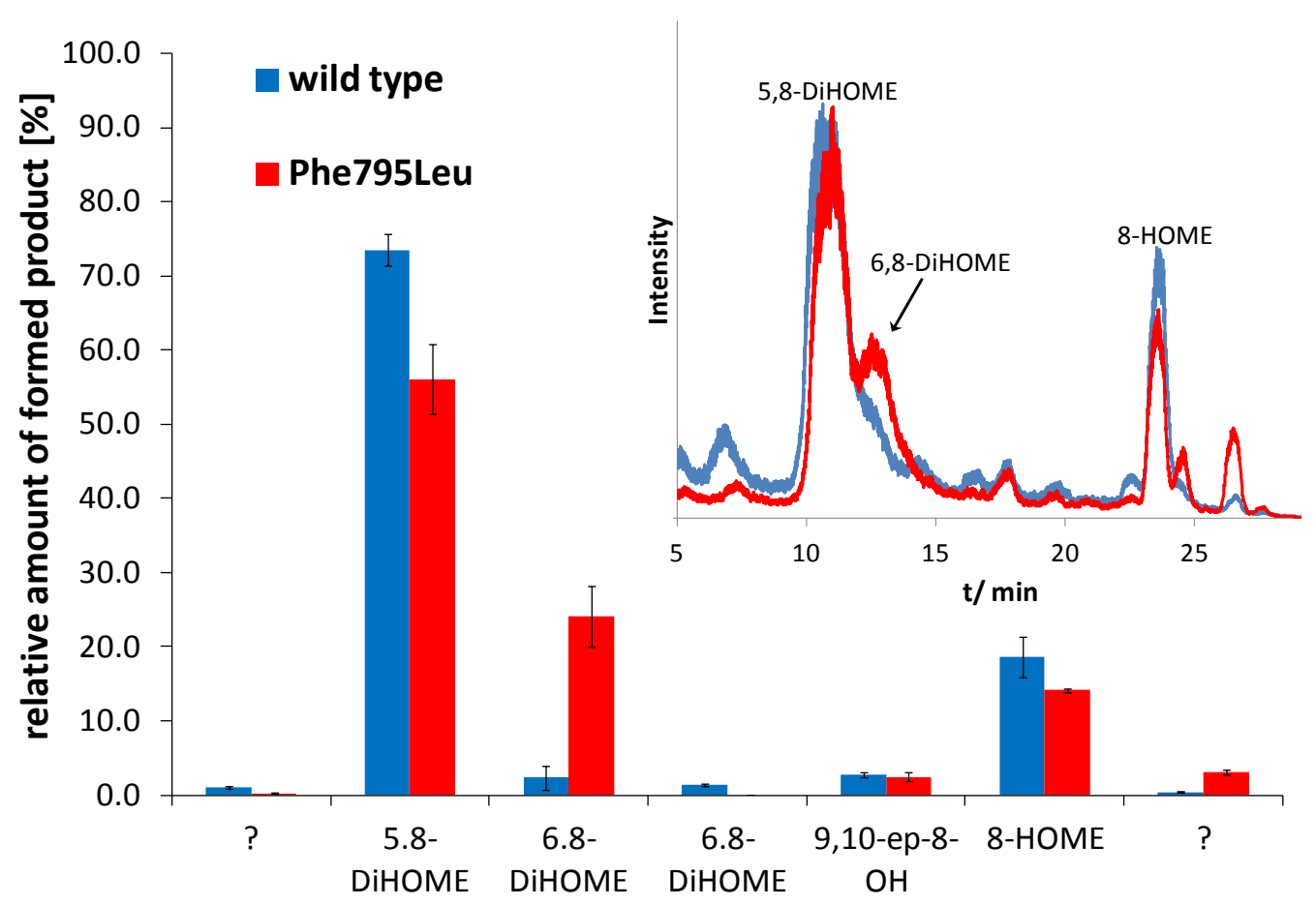

Figure 22: Phe795 in SRS22 determines the regioselectivity of PpoA's fatty acid isomerase activity. The conversion was conducted by incubation of $1 \mathrm{ml}$ cell lysate with $100 \mu \mathrm{M}$ oleic acid for $2 \mathrm{~min}$. The reaction was quenched and extracted twice by $E t_{2} O$. No significant difference in the overall amount of oxidized fatty acids was observed and product identity was verified by their $\mathrm{MS}^{2}$-spectra (Section 3.7). Note that two minor products have only been tentatively assigned as 8,11-DiHOME and 8-KOME and that two peaks have the MS/MS-spectrum of 6,8-DiHOME, representing most likely different diastereomers of this compound. Shown is the quantitative evaluation of three biological replicates each measured as triplicate as well as the corresponding standard deviation. Inset: Representative chromatograms of the conversion of oleic acid by wild type enzyme (blue) and the Phe795Leu-variant (red). 
In agreement with $\pi$-stacking between aromatic protein residues and the substrate's double bonds, conversion of linoleic acid, in which a second double bond might contribute to substrate binding, by the Phe795Leu-variant led to a less pronounced increase in the amount of byproduct as compared to oleic acid conversion. Still, an increase in the relative amount of 6,8DiHODE from $8 \%$ for the wild type enzyme to $16 \%$ for the variant was observed (Figure 23). To elucidate the putative involvement of Phe799's phenyl ring in binding of the $\Delta 12$ double bond, linoleic acid was converted by the respective variant. Strikingly, this variant showed a more severely disturbed product pattern than the Phe795Leu variant (Figure 23). Not only accumulation of products from the DOX-domain (8-HODE and 8-HPODE) indicated a reduced turn-over of the rearrangement reaction catalyzed by PpoA's cytochrome P450 activity, but also the nature of rearranged products was more diverse than observed so far and comprised dihydroxy derivatives (8,11-DiHODE; 5,8-DiHODE; 6,8-DiHODE) as well as epoxy alcohols (tentatively assigned as 8-hydroxy-9,10epoxy-12Z-octadecenoic acid and 10-hydroxy-8,9epoxy$12 Z$-octadecenoic acid). Additionally, two peaks were detected, which's fragmentation pattern was consistent with the spectrum of 8-KODE. Since the identification of ketones and epoxy alcohols indicated a non-enzymatic homolytic peroxide cleavage of 8-HPODE by free heme (Ernst $\mathrm{H}$ Oliw et al., 2006), the heme-occupancy of the P450-domain of the Phe799Leu-variant was investigated next.

A significantly decreased soret band of the purified variant in comparison to wild type enzyme indicated a loss of heme (Figure $24 \mathrm{~A}$ ). To attribute this lower heme occupancy to one of the two domains, a continuous wave X-band EPR-spectrum was recorded in collaboration with Alistair Fielding (MPI for Biophysical Chemistry, Goettingen; Figure $24 \mathrm{~B}$ ). The spectrum reveals that the low spin heme content of this variant is reduced to approx. $15 \%$ of the wild type's value, while the g-values are unaffected. This result indicates a heme loss without perturbation of the heme's coordination environment. Supporting this spectroscopic data, the purified Phe799Leu-variant showed a drastic decreased amount of $\mathrm{P} 450$-derived products and 70 to $90 \%$ of all products were derived from PpoA's DOX-domain (i.e. 8-HODE and 8-HPODE; Figure $24 \mathrm{C}$ ). Although these data suggest a loose binding of heme in the Phe799Leu-variant accompanied by heme-bleeding into the buffer, free heme-chemistry does not explain the diversification of the product-pattern in the purified variant. Hence, Phe799 seems not only to affect heme binding, but also reaction specificity. To probe the crucial property of the residue at position 799, further variants (Phe799Tyr; Phe799Trp and Phe799Met) were constructed. Interestingly, all of those variants did not show a reduced heme content in comparison to wild type enzyme (data not shown). Nevertheless, the Phe799Met-variant showed the same diversified product pattern as the Phe799Leu-variant, additionally indicating that the diversification of products rearranged from 8-HPODE might not be crucially governed by free heme, but rather by an unconstrained substrate binding to the active site of PpoA's P450-domain (Figure 25). In line with the idea that the residue at amino acid position 799 is involved in substrate binding is the observation that a bulky tryptophan at this position prevents isomerization of 8-HPODE to a great extent, 
potentially by blocking the active site. Not that surprising is the observation that the Phe799Tyrvariant exhibited a nearly identical product pattern as the wild type enzyme. Still one can see from the obtained data that even with such a slight modification of the amino acid at position 799 a significant decrease in hydroperoxy fatty acid isomerase activity is associated. Considering that the rate-limiting step of the overall reaction is hydrogen-abstraction in the DOX-domain (Section 4.7), this activity reduction becomes even more evident. Despite the data obtained here, the question, whether the roles of Phe795 and Phe799 in determining reaction specificity can be attributed to be a result of improper substrate placement, due to a lack of $\pi$-stacking between the substrate's double bonds and the aromatic amino acid side chains, or whether the observed effects have to be explained by other means, is not finally answered by these experiments. However, in this respect it is mentionable that the Phe799Leu-variant, which was proposed to exhibit an impaired ability to correctly place the $\Delta 12$ double bond, is also rearranging oleic acid to a diversified product pattern (data not shown).

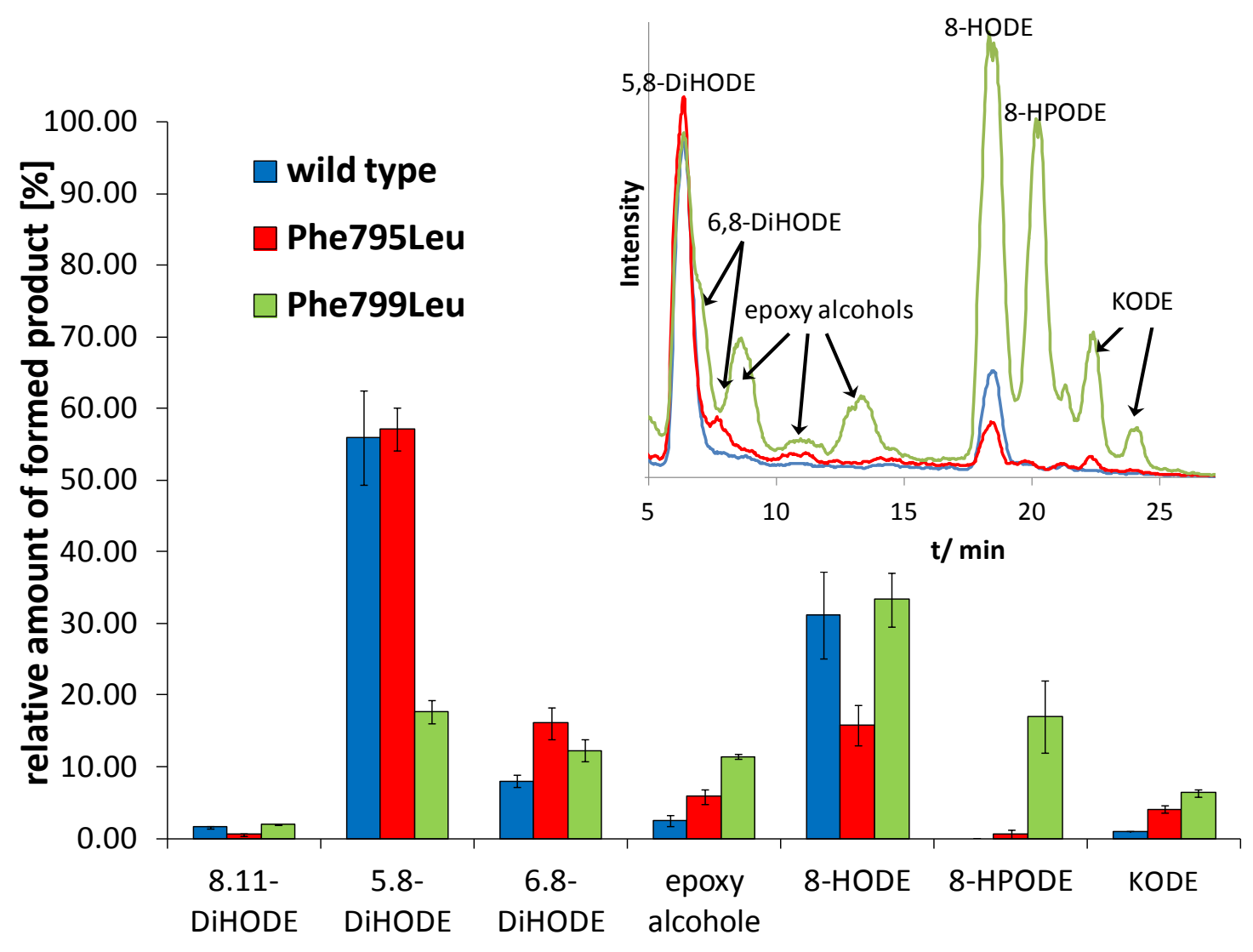

Figure 23: Phe795 in SRS2 and Phe799 in SRS3 determine the regioselectivity of PpoA's fatty acid isomerase activity. The conversion was conducted by incubation of $1 \mathrm{ml}$ cell lysate with $100 \mu \mathrm{M}$ linoleic acid for $2 \mathrm{~min}$. The reaction was quenched and extracted twice by $\mathrm{Et}_{2} \mathrm{O}$. Products were identified by their $M S^{2}$-spectra (Section 3.7). Shown is the quantitative evaluation of three biological replicates as well as the corresponding standard deviation. Inset: Representative chromatograms of the conversion of linoleic acid by wild type enzyme (blue), the Phe795Leu-variant (red) and the Phe799Leu-variant (green). Note that peaks with the same assignment have identical MS/MS-spectra representing most likely different isomers of this compound. 

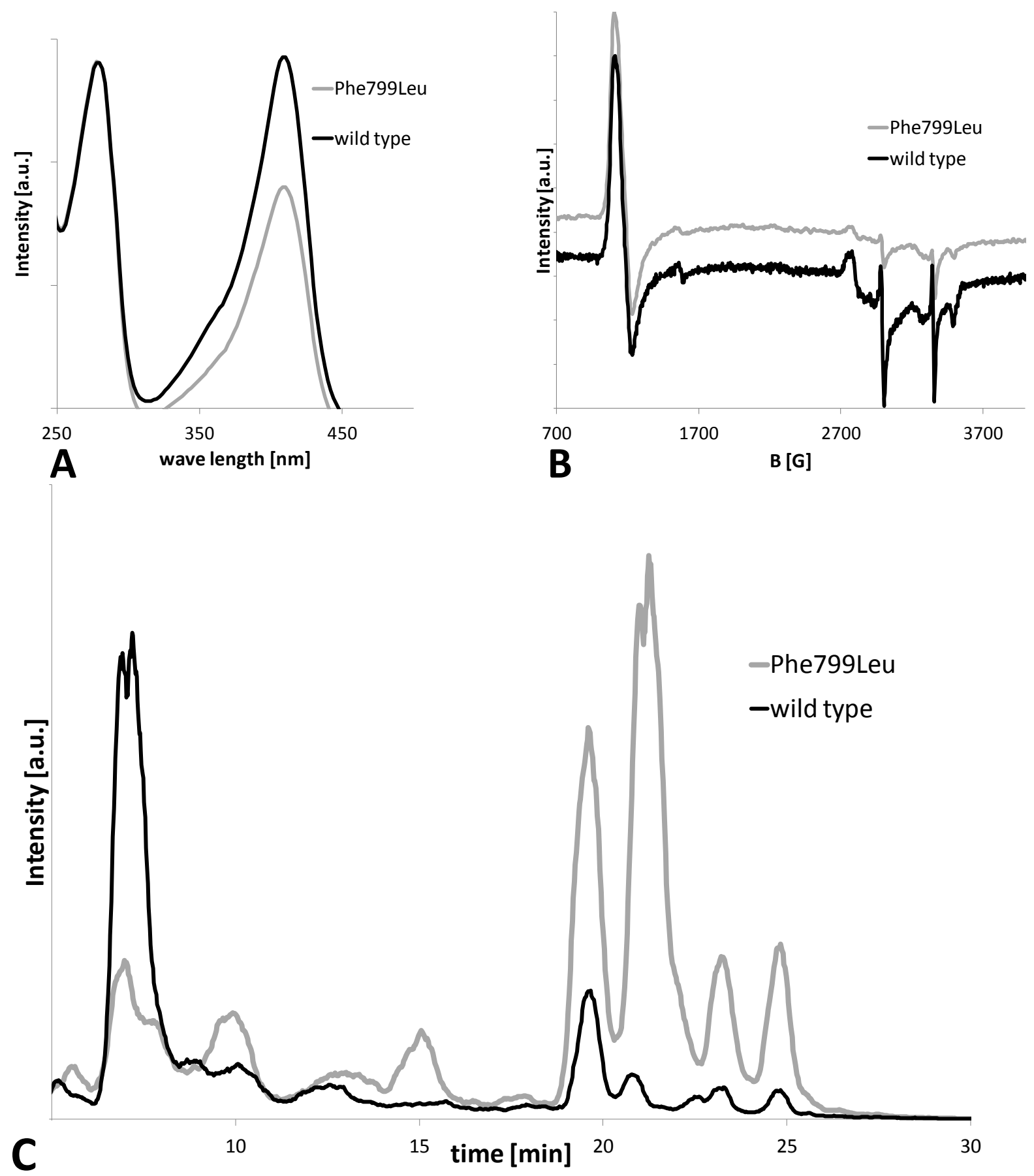

Figure 24: The mutation of Phe799 to Leu leads to a significant loss of cytochrome P450-heme of PpoA. A The UV/VIS-spectrum of this variant shows a reduced soret-peak relative to wild type enzyme. This indicates $a \sim 40 \%$ heme loss. B X-band CW-EPR spectrum of the variant shows that only the low-spin heme content (i.e. the P450-heme (Fielding et al., 2011)) is reduced, while the high-spin heme (i.e. the DOX-heme (Fielding et al., 2011)) shows the same abundance as in the reference spectrum of wild type enzyme. Note that for clarity an offset was added to the variant's spectrum. C Representative LC/MS-chromatogram of linoleic acid conversion by purified PpoA_Phe799Leu. Note that the amount of rearranged 8-HPODE is drastically reduced in comparison to wild type enzyme and a concomitant accumulation of 8-HPODE is observed. Three independent conversions were performed. 

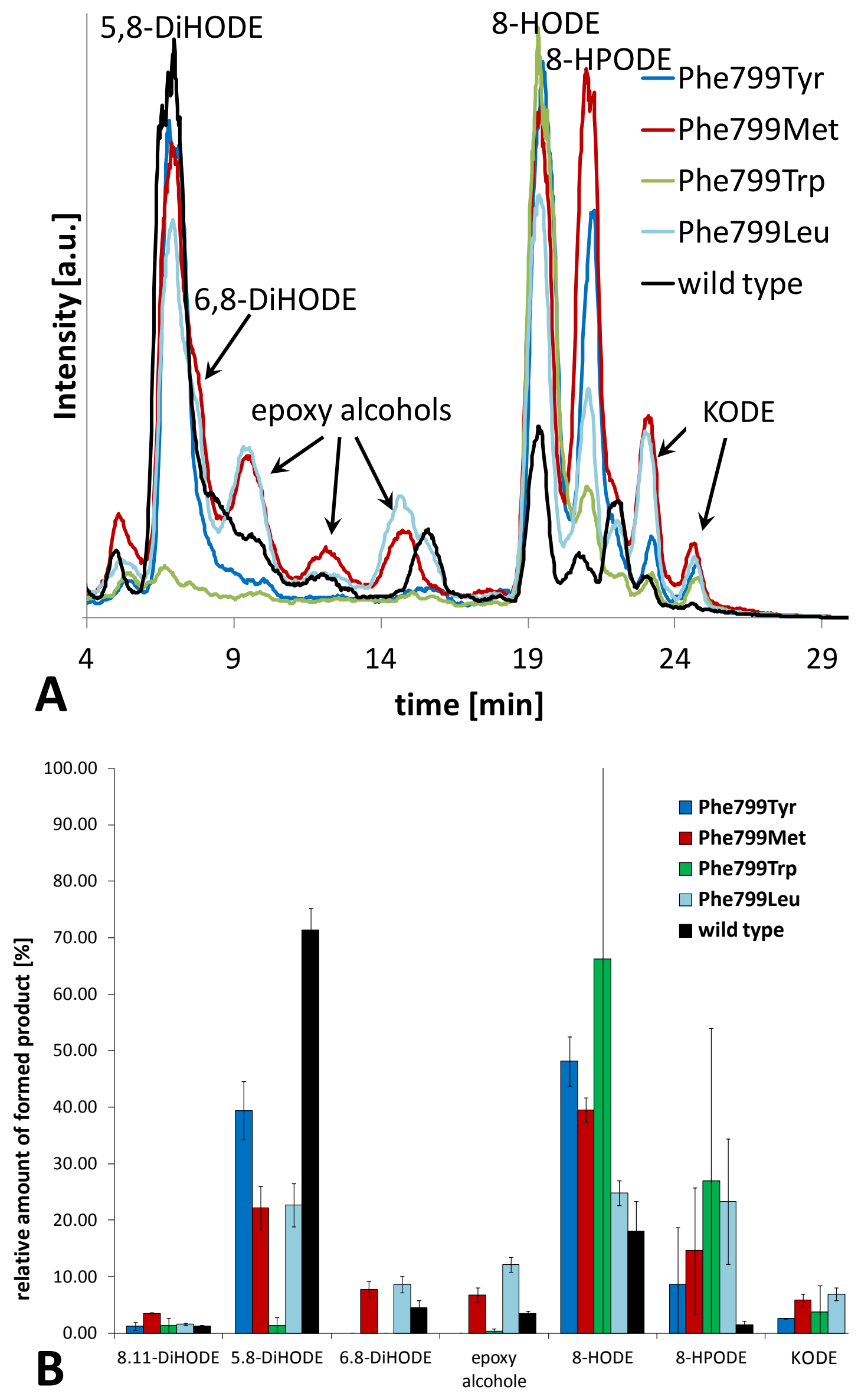
Figure 25 (previous page): Product patterns of linoleic acid conversion by PpoA_Phe799Leu, PpoA_Phe799Tyr, PpoA_Phe799Trp, and PpoA_Phe799Met in comparison to wild type enzyme. $100 \mu \mathrm{M}$ linoleic acid was converted by the respective cell lysates. While the Trp-variant rearranged basically no hydroperoxy fatty acid, the Tyr-variant showed a product pattern reminiscent of the wild type pattern, although the activity of the P450-domain was reduced. In contrast, the leucine-and methionine-variants showed a diversified product pattern. A Representative chromatograms. B Quantitative evaluation of LCMS data. The patterns were obtained from three independent enzyme expressions of each variant. Shown is the arithmetic mean as well as the corresponding standard deviation.

\subsubsection{ASPARAGINE 887 IS CRUCIALLY INVOLVED IN HYDROPEROXIDE REARRANGEMENT}

It is known that the amide function of an asparagine in the I-helix of hydroperoxy fatty acid rearranging P450s is crucially involved in peroxide cleavage (Chiang et al., 2006; D.-S. Lee et al., 2008). Notably, sequence alignments did not lead to identification of a homologue residue in PpoA and in the initial model of the P450-domain, no asparagine was at the expected position. However, a neighboring residue within the I-helix is an asparagine (Asn887), but its side chain points by approximately $90^{\circ}$ away from the expected direction. Assuming that this detail might be false predicted, a respective variant (Asn887Val) was constructed. Although the conducted variation did not lead to a qualitative change of the product pattern and only the three enzymatic products known from conversions by wild type enzyme, i.e. 8-HODE, 8-HPODE and 5,8-DiHODE, were produced, there was a significant effect on the hydroperoxide isomerase activity causing a decrease in the relative amount of rearranged product and a concomitant accumulation of DOX-derived products (Figure 26).

During characterization of the purified PpoA-variant, it became obvious that the conducted mutation leads additionally to a reduced soret absorption of the enzyme. To address whether this reduced vis-absorption is caused by a lower heme-content of either the DOX-or the P450domain, or merely reflects altered cofactor coordination in one or the other domain, Dr. Alistair Fielding (MPI for Biophysical Chemistry, Goettingen) recorded cw X-band EPR-spectra of the variant as well as of wild type enzyme. The obtained data reveal that there is no difference in the high-spin heme indicating that the cofactor of the DOX-domain is not affected by the conducted variation. In contrast, the low-spin signal assigned as P450-heme (Fielding et al., 2011) was not only reduced by approximately $40 \%$, but also the g-tensor was altered (Figure 27). Since the gvalues of a P450 enzyme are very sensitive to the cofactor's ligation sphere, these changes might indicate an altered coordination as one would expect, if the amide of Asn887 forms a hydrogen bonding network involving the subtrate's peroxide and the heme-iron. 
Results

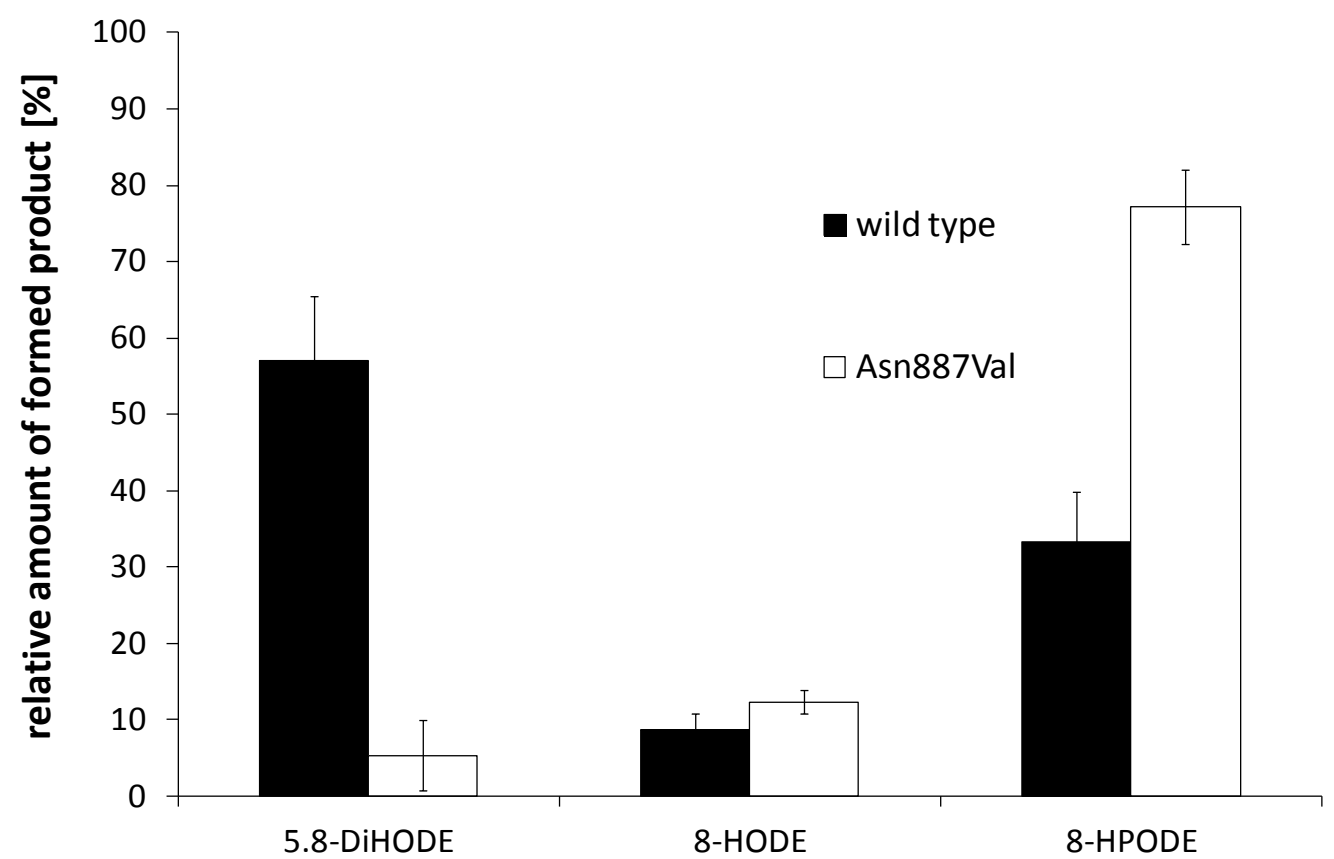

Figure 26: The Asn887Val-variant of PpoA exhibits a significantly reduced hydroperoxy fatty acid isomerase activity as compared to the wild type. This effect is revealed by a decreased amount of rearranged product and a concomitant accumulation of DOX-derived products. The reaction of the respective variant with linoleic acid was extracted after $2 \mathrm{~min}$ and product patterns in these extracts were measured by LC-MS'. Shown are the arithmetic means of products quantified for three independent conversions as well as the corresponding standard deviation.

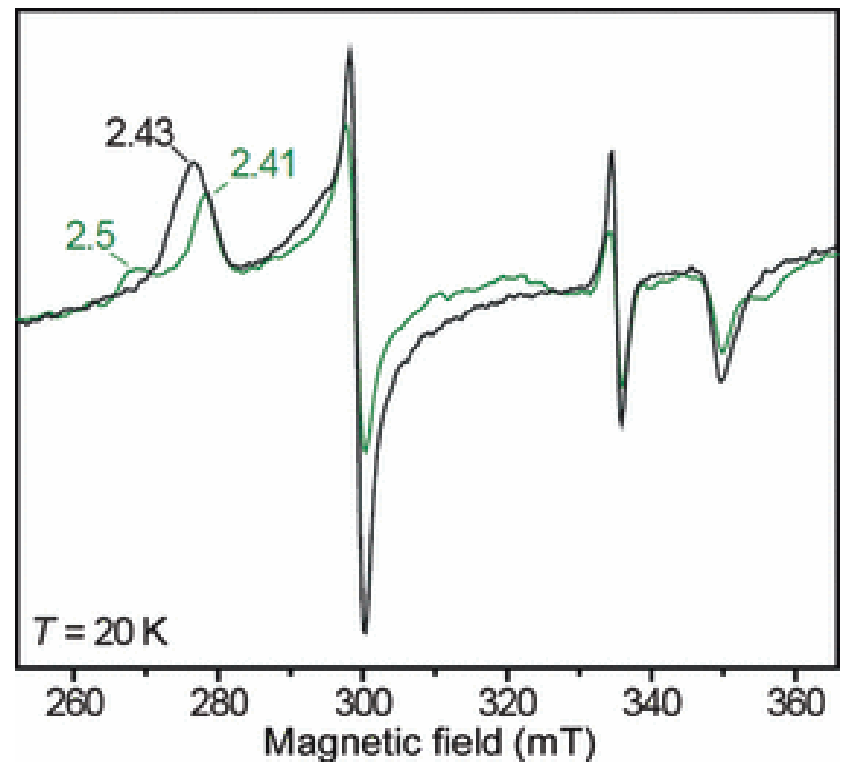

Figure 27: $\mathrm{cW} X$-EPR spectra of the low-spin heme species in PpoA-Asn887Val (75 $\mu \mathrm{M}$; green) as compared to wild type (75 $\mu \mathrm{M}$; black). Qualitative differences in $g_{1}$ are marked. Additionally, the heme occupancy in the variant is only about $60 \%$ of the wild type value. Spectra were recorded by Dr. Alistair Fielding with details specified in literature (Fielding et al., 2011). The spectrum of the variant was measured for a single preparation and the wild type spectrum is consistent with spectra obtained for several other preparations of this enzyme 
A final experiment to shed light on the role of asparagine 887 was based on the idea that the asparagine might structurally resemble the functional homologue position in class III P450s. As it was shown for PpoA (Brodhun et al., 2009), this P450 class in general exhibits only weak and transient binding of typical P450-heme ligands like CO and imidazole (Yeh et al., 2005; D.-S. Lee et al., 2008). Taken crystallographic data into account, this observation is explained by the carboxamide of the catalytic asparagine sterically hindering the direct coordination of small ligands to the heme iron (D.-S. Lee et al., 2008). To probe a similar position of Asn887 directly above the distal heme plane in PpoA's P450-domain, an imidazole titration was performed as already outlined in section 4.2.5 and figure 14. Briefly, wild type enzyme and the Asn887Valvariant were both titrated with imidazole and the red-shift of the soret band was monitored. Interestingly, titrations of both enzymes yielded basically the same dissociation constant ( $\mathrm{k}_{\mathrm{D}} \sim 6$ to $11 \mu \mathrm{M}$, assuming a one-site binding model), indicating that Val still might be too bulky to permit access to the heme or that the asparagine in PpoA indeed is farer apart from the heme than in other class III P450s, as it was predicted in the structural model (Section 4.3.1.2). Notably, the measured affinity is roughly 50-times higher as it was reported for prostacyclin synthase (Yeh et al., 2005), which might additionally point out a more accessible heme. By imidazole titration of PpoA's His1004Ala-variant, in which the P450-heme is absent (Brodhun et al., 2009), it could be shown that also the histidine coordinated heme of the DOX-domain is binding imidazole. Thus, for proper evaluation of the spectral binding assays, consideration of a two-site binding model was necessary. Therefore the binding parameters obtained for titration of the His1004Alavariant $\left(k_{D} \sim 34 \mu \mathrm{M}\right)$ were defined as (fixed) parameters of imidazole binding to the DOX-domain and affinity parameters of imidazole binding to the P450-heme were subsequently calculated from titrations of wild type enzyme and the Asn887Val-variant, respectively, with fixed parameters for imidazole binding to the DOX-heme and the imidazole binding parameters for the P450-heme fitted to a two-site binding model. For both, the wild type enzyme and its Asn887Val-variant, the thus obtained $K_{D}$ did not differ significantly and were in the range of $\sim 80$ $\mu \mathrm{M}$. This value still represents an about 6-fold higher affinity of imidazole to the P450-heme of PpoA as compared to prostacyclin synthase. Again the missing influence of a less bulky residue at position 887 on ligand access supports the idea that this position in PpoA is less important in shielding of the P450 heme as in other class III P450s.

Taken together the here presented data substantiate the involvement of Asn887 in hydroperoxide rearrangement and justify the refinement of the initially obtained predicted structure. Nevertheless, the heme-binding of imidazole suggests that the P450-heme of PpoA is shielded to a lesser extent than in other class III P450s. This in turn might indicate that the catalytic competent asparagine, which is responsible for the steric shielding, has a different position and is in agreement with the failure to identify a catalytic competent asparagine by sequence alignments. 


\subsection{Attempts to convert 5,8-LDS to 7,8-LDS}

Psi-factor producing oxygenases in Aspergillus nidulans have been identified by homology to the enzyme 7,8-linoleate diol synthase (7,8-LDS) from Gaeummanomyces graminis, which synthesizes 7,8-dihydroxy fatty acids (Tsitsigiannis, Zarnowski, et al., 2004). In the following years several in depth studies have shown that both enzymes posses the same domain-structure comprising of an N-terminal DOX-domain and a C-terminal cytochrome P450, which represents a conserved architecture that is common to all members of the LDS-family. It was shown for 7,8LDS that the $\mathrm{N}$-terminal domain catalyzes the same reaction as PpoA and converts an unsaturated $18 \mathrm{C}$ fatty acid to its 8-hydroperoxy derivative (Garscha and Ernst $\mathrm{H}$ Oliw, 2008). Also the alignment of both sequences illustrates that the determinants for the different regioisomers synthesized as products of the respective enzyme are most likely located in the P450-domain.

In principle two possible explanations for the changed regioselectivity of both hydroperoxy fatty acid isomerases seem possible: i) a change of the protein architecture at the distal side of the heme leads to a sterically caused readjustment of the substrate position or ii) an amino acid exchange within the heme binding loop leads to a modification of the heme's redox potential causing a changed kinetics or mechanism of heme-chemistry.

\section{i) Is regioselectivity determined by a changed substrate binding?}

As already shown in section 4.4 .3 , Phe795 seems to contribute via $\pi$-stacking with the $\Delta 9$ double bond of the substrate to the placement of this substrate in a proper position. Knowing that the substrate will not undergo severe reorientations between heterolytic peroxide cleavage and hydroxylation of $C_{5}$ [40], it is reasonable to assume that a transition state will be formed at which the two carbons involved in the reaction (i.e. $\mathrm{C}_{8}$ and $\mathrm{C}_{5}$ ) are concomitant in close proximity of the heme iron (Figure $28 \mathrm{~A}$ ). From the structure, one can deduce that the long aliphatic side chain of Met791 might push $\mathrm{C}_{5}$ towards the iron, while a small amino acid at position 792 could create a cavity that allows $C_{6}$ and $C_{7}$ to be placed in unproductive distance from the cofactor (Figure 28A). The corresponding positions in G. graminearum are Leu796, which is less bulky than Met and hence would not force $C_{5}$ to be placed next to the iron, and Val797, slightly bulkier than Ala792 in PpoA and thus could push substrate's $C_{7}$ in a productive distance to the heme (Figure $28 \mathrm{~B}$ ). Nevertheless, the corresponding variant of PpoA as well as the double mutant (PpoA_Met791Leu and PpoA_Met791Leu/Ala792Val) showed an identical product pattern compared to the wild type and also the variant resembling the situation of Magnaporthe grisea (PpoA_Met791Leu/Ala792Met), which was thought to more likely push $C_{7}$ in a productive distance to the heme, did not show an altered product pattern (data not shown). 


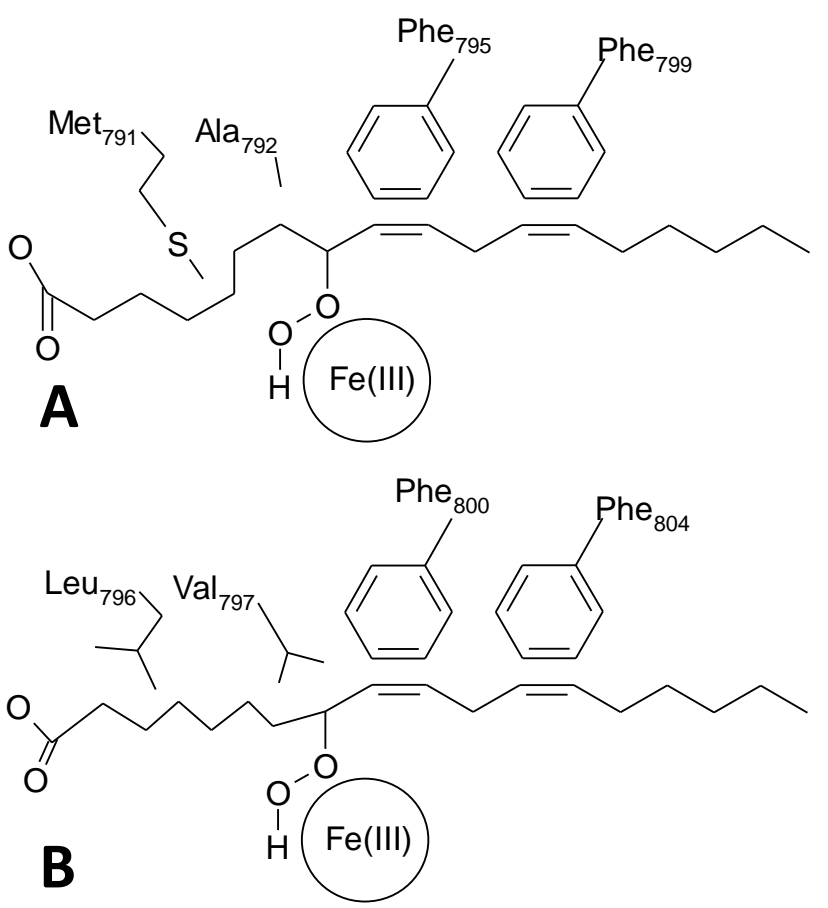

Figure 28: Proposed transition state of the substrate bound to PpoA's P450-domain and thereof derived determinants for regioselectivity. $\boldsymbol{A}$ illustrates the situation in 5,8-LDS (i.e. PpoA), while $\boldsymbol{B}$ depicts the equivalent positions in Gaeummanomyces graminis 7,8-LDS. Note that 7,8-LDS of Magnaporthe grisea (Cristea et al., 2003) has a situation with Met instead of Ala792 and Leu instead of Met791, making the explanation for formation of 7,8-DiHODE more likely.

\section{i) Does an altered heme-chemistry influence regioselectivity?}

There are only some known determinants that govern the redox potential and therefore the reactivity of the heme cofactor of an enzyme. Of those determinants the proximal ligand coordinating the iron is by far the most important one (Thomas L. Poulos, 1996). Besides the proximal ligand, only a few other amino acids have been implicated to modulate the cofactor's redox potential. Matsumura et al. identified two residues in the heme binding loop and showed their influence on the redox potential (Matsumura et al., 2008). While the phenylalanine at the beginning of the P450 consensus sequence, seven amino acids upstream of the iron's cysteine ligand is invariant in 7,8- and 5,8-LDS, the second identified determinant differs. The hydrophobic leucine three amino acids downstream the cysteine ligand of PpoA is replaced by a charged glutamate in 7,8-LDS. Matsumura et al. have shown that replacement of a hydrophobic amino acid (Ala) at this position by a hydrophilic one ( $G(n)$ leads to a decreased electron density on the heme-iron and therefore directly influences the kinetics of compound I formation (Matsumura et al., 2008). A decreased rate of compound I formation could in turn favor a reorientation bringing carbon 5 to the oxidizing species in 5,8-LDS, while a faster formation of this oxidizing species in 7,8-LDS might promote instant oxidation of the neighboring carbon 7. To address the question whether the variation of this position plays a role in the differentiation between 5,8- and 7,8-LDS, the respective variant of 5,8-LDS (PpoA_Leu1009Glu) was generated. However, also this enzyme variant exhibited virtually the same product pattern as the wild type. 
Results

\subsection{Quaternary structure of PpoA}

\subsubsection{Measuring the native low-resolution structure of PpoA by SaXs}

To derive a hypothesis how the independently predicted domains might interact with each other and make up an oligomeric enzyme, a low resolution structure of the native enzyme was determined by SAXS. Small-angle scattering data were obtained in collaboration with Giancarlo Tria (BioSAXS-group, EMBL, Hamburg) for six different concentrations (48.8 g/l, $25.0 \mathrm{~g} / \mathrm{l}, 10.5 \mathrm{~g} / \mathrm{l}$, $5.8 \mathrm{~g} / \mathrm{l}, 3.0 \mathrm{~g} / \mathrm{l}$ and $2.0 \mathrm{~g} / \mathrm{l}$ ) of the wild type enzyme (in $50 \mathrm{mM}$ HEPES, pH 7.4 with or without 2 mM DTT addition) at the X33-beamline at the DESY in Hamburg. The resulting scattering curves and Guinier plots indicated that the enzyme preparation showed no aggregation tendency and was highly monodisperse. Also, addition of $2 \mathrm{mM}$ DTT, which can be used in order to reduce aggregation, did not alter the scattering curve. The distance distribution function suggested a maximal particle's dimension of $\sim 16.5 \mathrm{~nm}$, a radius of gyration of $\sim 5.4 \mathrm{~nm}$ and an occupied volume of $\sim 546 \mathrm{~nm}^{3}$. This directly measured particle volume suggested a molecular weight of $\sim 341 \mathrm{kDa}$, which would correspond to a trimeric quaternary enzyme structure of the enzyme (the monomer has a theoretically calculated molecular weight of $\sim 120 \mathrm{kDa}$, while its apparent molecular weight judged by SDS-PAGE is $\sim 110 \mathrm{kDa}$ ). The $a b$ initio determined molecular shape that best describes the experimentally observed scattering curve was calculated by Giancarlo Tria utilizing the program DAMMIF (D. Franke and Svergun, 2009). Interestingly, the thus determined shape (Figure $29 \mathrm{~A}+\mathrm{B}$ ) agreed with the low resolution structure obtained by rigidbody docking of three monomers of each of the previously modeled enzyme domains to the scattering curve using the SASREF algorithm (Petoukhov and Svergun, 2005) (Figure 29 C+D). The thus obtained structure showed an excellent agreement with the experimental data.

To validate the derived model of the enzyme's quaternary structure, surface exposed and accessible protease cleavage sites were mapped. Therefore the protein was subjected to native proteolysis with trypsin. Although size exclusion chromatography indicated that the native molecular weight was unchanged, this treatment yielded reproducible four stable fragments that could be separated by SDS-PAGE (Figure $30 \mathrm{~A}$ ). The sites that were accessible to this treatment were determined by ESI-MS ${ }^{2}$ of the excised bands (measurements by Dr. Oliver Valerius, Georg-August-University, Goettingen). Although this approach was complicated by the generally incomplete peptide-coverage on the one side and a highly sensitive detection of unspecifically truncated peptides forming a background over the whole lane on the other side, four sites of truncation have been identified (Arg140; Lys350; Lys806, and Lys834) and were highlighted within the enzyme's model (Figure $30 \mathrm{~B}$ ). Clearly, the accessible digestion sites are distributed on the edge of the molecule and the flat sites of the particle are inaccessible for the protease's active site. 

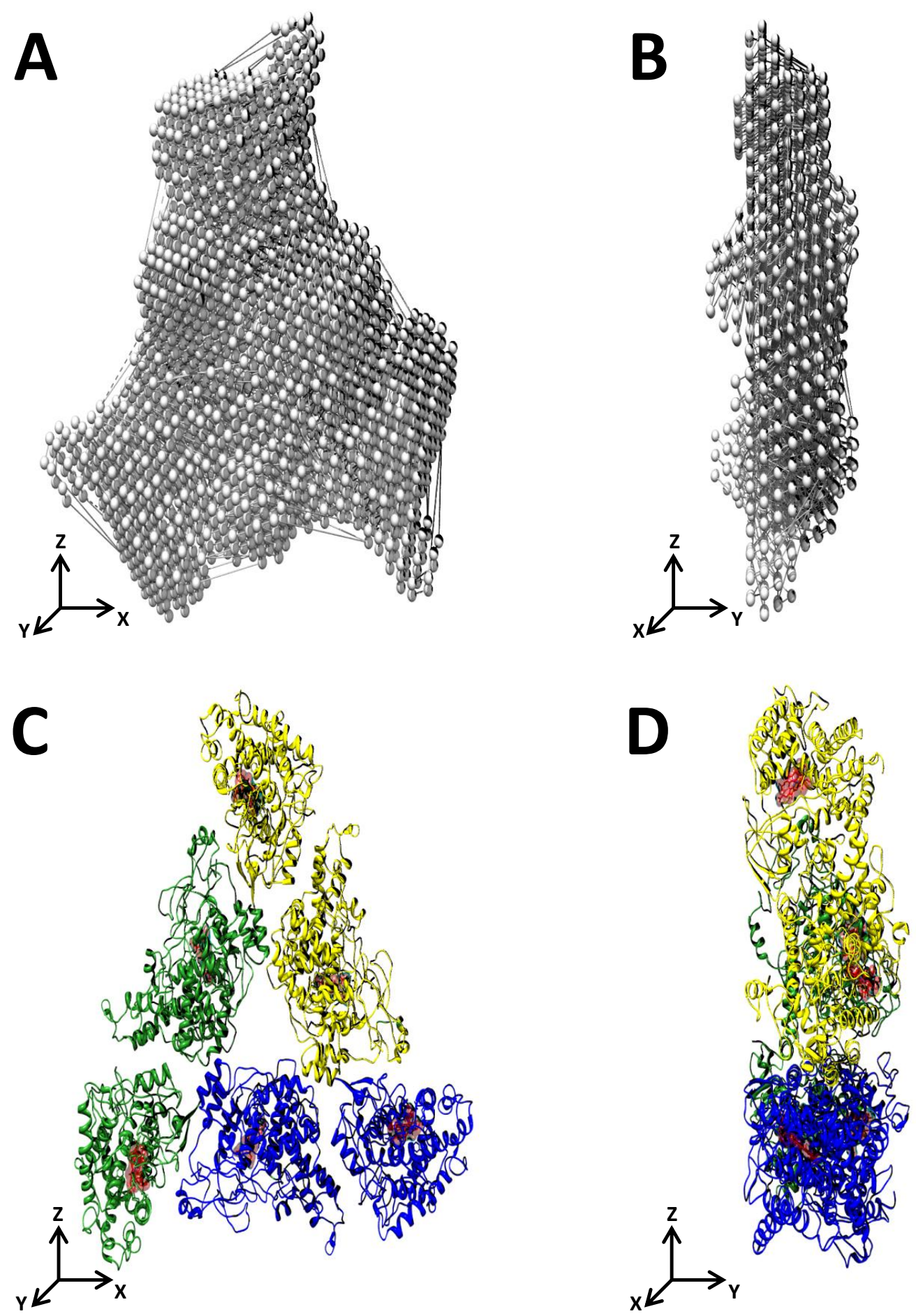

Figure 29: PpoA's quaternary structure derived from a small-angle $X$-ray scattering intensity profile of native wild type enzyme. $\mathrm{A} A \mathrm{Ab}$ initio determined bead-model of the particle shape that fits the experimentally observed scattering curve. The shape was calculated by EMBL's BioSAXS group using the DAMMIF algorithm. $B 90^{\circ}$ rotation of $A$ around the z-axis. C Rigid-body docking of three PpoA-monomers (i.e. three predicted DOX- and three predicted P450-domains) to the scattering curve. The docking was calculated in EMBL's BioSAXS group using the SASREF algorithm. $D 90^{\circ}$ rotation of $C$ around the z-axis. 


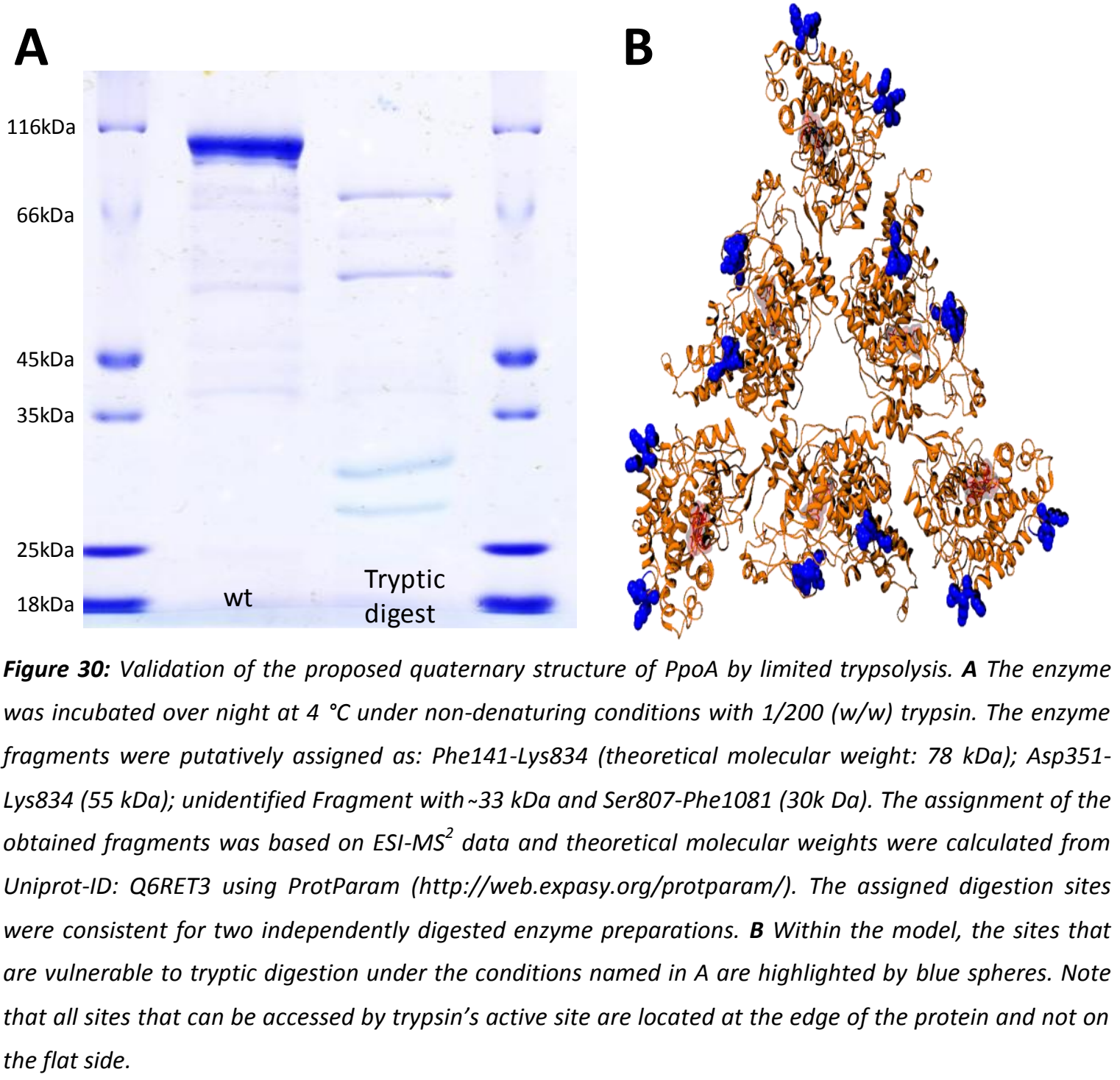

\subsubsection{Measuring tyrosyl-Radical Distances by DEER}

DEER is a technique to probe the distance between two paramagnetic centers. Since it is known that the first step of oxygen insertion is mediated by such a paramagnetic center, i.e. a tyrosylradical that abstracts hydrogen from the fatty acid's $C_{8}$, one can measure the distances between these tyrosyls in the different monomers of the protein and use this constraints to further evaluate the proposed quaternary enzyme structure. From the obtained low resolution structure of PpoA (Section 4.6.1), a distance of the proposed radical-forming Tyr374 of $5.9 \mathrm{~nm}$ was derived. However, one has to keep in mind that SAXS will yield merely low-resolution structures and thus these distances are associated with an experimental error of up to $2 \mathrm{~nm}$. Independently from their true spacing, the arrangement of the monomers within a triangular quaternary structure gives rise to an equilateral distance triangle between active site residues of the distinct domains (Figure 31). In order to measure the distances between the catalytic active tyrosines with a more precise method and thus verify or falsify the SAXS-derived enzyme structure, DEER 
was employed. Interestingly, Fielding et al. measured already the distance between the catalytic active tyrosyl radicals and determined one significant distance of $5.2 \mathrm{~nm}$ (Fielding et al., 2011). Although this distance is in agreement with the spacings of the Tyr374s determined from the SAXS-structure and the existence of merely one significant distance can be explained by the spatial arrangement of the three tyrosyl radicals as vertices of an equilateral triangle, an additional, minor distance extracted in this study complicates the picture again. Considering that Tyr327 is a second tyrosine residue within the DOX active site and is in reasonable distance to the substrate carbon to be oxidized, this distance $(3.9 \mathrm{~nm})$ could be explained by an additional radical site at this tyrosine (Figure 31). To evaluate this possibility, the tyrosyl radical distances in the Tyr327Phe-variant were determined additionally to those of the wild type enzyme.

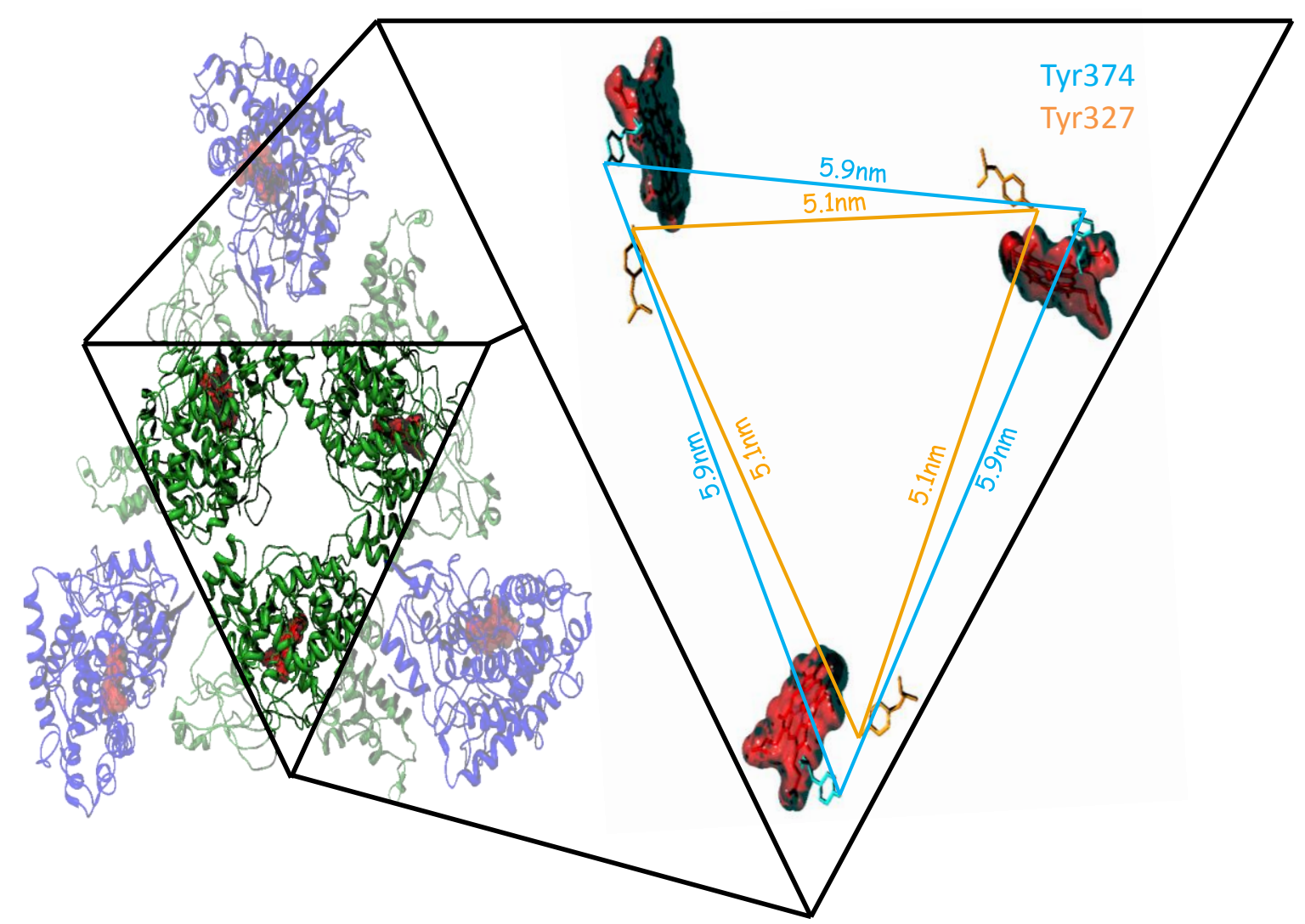

Figure 31: Zoom into the DOX active-sites. Tyrosine residues putatively forming the substrate oxidating radical are shown. The distances between the three Tyr374-sites (cyan) are $5.9 \mathrm{~nm}$, while the distances between the alternative Tyr327-sites (orange) are $5.1 \mathrm{~nm}$. Within the Structural model of PpoA, DOXdomains are colored green and the P450-domains are colored blue. Hemes are rendered in red. 
Taking advantage of the superior signal to noise ratio and a lower demand on sample amount (Ghimire et al., 2009), we intended to extend the previous study on this issue at X-band (Fielding et al., 2011) and obtain the new data at Q-band. In order to measure the expected distance by DEER, the dipolar evolution time trace should be recorded for at least $2.5 \mu \mathrm{s}$ (G. Jeschke et al., 2004). While this evolution time is sufficient to obtain a reliable distance-value, longer evolution times are favorable in order to extract exact distance distributions. Besides the problem to generate and trap a sufficient amount of tyrosyl radical, the main obstacle for the given analytical task is the rather small phase memory time of the generated radical. This enhanced relaxation might be caused by the proximity to the heme iron (Fielding et al., 2011). To decrease these relaxation processes, the influence of glycerol and $\mathrm{D}_{2} \mathrm{O}$ on relaxation times was assessed (Figure 32). Therefore, $100 \mu \mathrm{M}$ of wild type trimer was reacted with an equal volume of $12 \mathrm{mM}$ 8 -HPODE in the respective buffer. The reaction was immediately (reaction time 4 to 6 seconds) transferred to Q-band tubes, quenched in a dry ice bath and then stored in liquid nitrogen. Measurements of relaxation rates were performed at $6 \mathrm{~K}$ with the details given in Section 8.1.21. Interestingly, the utilization of $\mathrm{D}_{2} \mathrm{O}$ instead of water led to an unexpected enhanced relaxation of the radical. This might indicate that the rotational freedom of the tyrosyl is restricted by a hydrogen-bonding network involving water and thus is increased in $\mathrm{D}_{2} \mathrm{O}$. The addition of $20 \%$ glycerol did not lead to a significant increase of the decisive $T_{m}$, but contrary substantially lengthened $T_{1}$, which implements the requirement of a longer shot repetition time and thus a longer acquisition time in order to get the same number of spectral averages. Although a further increased glycerol concentration led to an even longer spin-lattice relaxation, the buffer condition containing $30 \%$ glycerol also positively affected the phase memory time and increased this parameter by $\sim 50 \%$. Since the formation of a glass instead of a crystalline frozen solution can advantageously influence relaxation times (Kveder et al., 2008), also a condition that allows glass-formation upon freezing was utilized. This sample contained $50 \mathrm{mM} \mathrm{HEPES,} \mathrm{pH} \mathrm{7.5,} \mathrm{with} 30$ $\%$ glycerol and $1 \mathrm{M}$ sucrose as vitrification buffer. However, this condition did not improve $\mathrm{T}_{\mathrm{m}}$ as compared to the buffer containing $30 \%$ glycerol, but prolonged $\mathrm{T}_{1}$. 


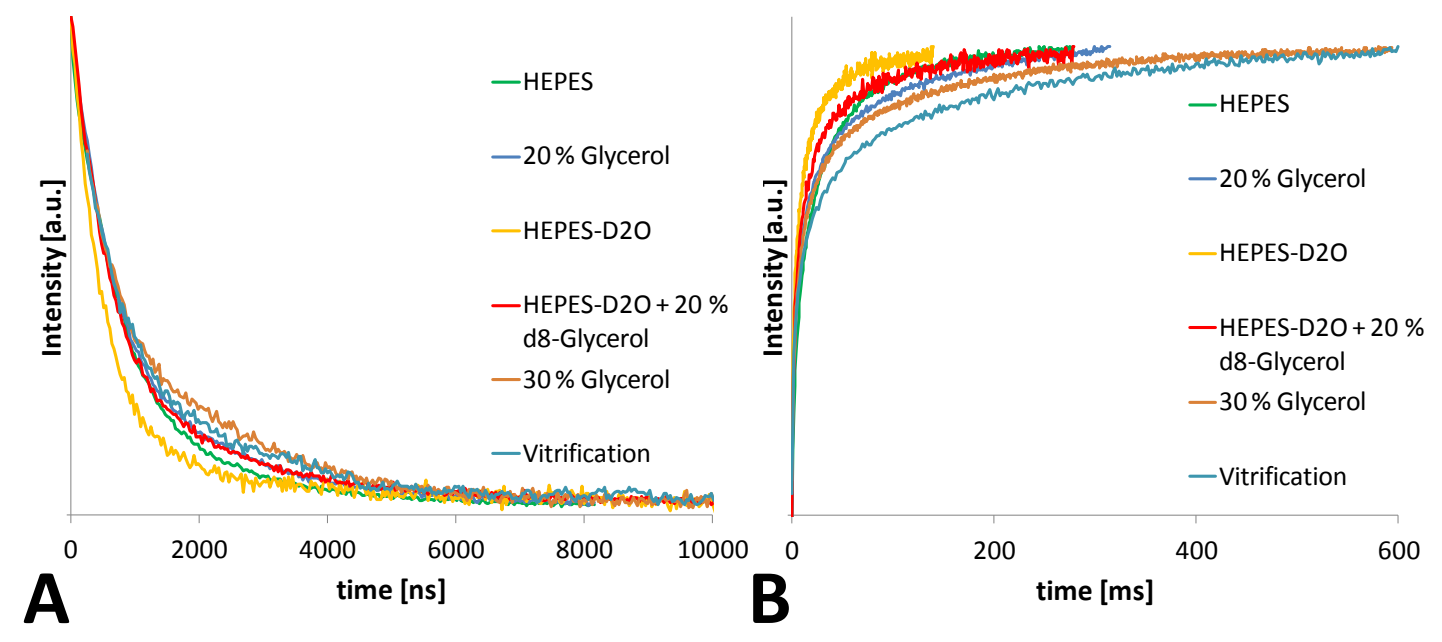

Figure 32: Relaxation rates of PpoA's tyrosyl radical under different buffer-conditions at $6 K$. $T_{1}(\boldsymbol{A})$ was measured by an inversion recovery sequence and $T_{m}(B)$ by a two-pulse decay. The obtained traces were fitted with an exponential $\left(T_{m}\right)$ and biexponential $\left(T_{1}\right)$ function, respectively. From these fits the following relaxation rates were obtained: $T_{1}($ HEPES $)=52.4 \mathrm{~ms} ; T_{m}($ HEPES $)=988 \mathrm{~ns} ; T_{1}(20 \%$ Glycerol $)=71.2 \mathrm{~ms}$; $T_{m}(20 \%$ Glycerol $)=984 n s ; T_{1}\left(H E P E S-D_{2} O\right)=21.9 m s ; T_{m}\left(H E P E S-D_{2} O\right)=647 n s ; T_{1}\left(H E P E S-D_{2} O+20 \% d_{8^{-}}\right.$ Glycerol $)=44.3 \mathrm{~ms} ; T_{m}\left(\right.$ HEPES- $D_{2} \mathrm{O}+20 \% \mathrm{~d}_{8}$-Glycerol $)=962 \mathrm{~ns} ; T_{1}(30 \%$ Glycerol $)=121 \mathrm{~ms} ; T_{m}(30 \%$ Glycerol) $=1470 \mathrm{~ns} ; T_{1}$ (vitrification) $=196 \mathrm{~ms} ; T_{m}$ (vitrification) $=1500 \mathrm{~ns}$. "HEPES" is short for $50 \mathrm{mM}$ HEPES, pH 7.5, and "vitrification" denotes a condition containing 50 mM HEPES, pH 7.5/ 1 M sucrose/30\% glycerol. In contrast to all other conditions in which the water crystallizes upon freezing, this buffer composition is supposed to give a perfect glass. All measured relaxation-rates were obtained with a single enzyme preparation.

Besides a reasonable phase-memory time, the second requirement in order to obtain highquality DEER-data is to generate a sufficient amount of radical. According to the values reported by Fielding et al. (Fielding et al., 2011), one could expect 0.6 spins per enzyme-trimer for manual mixed samples. To assess the influence of preparing the sample in X-and Q-band-tubes, respectively, as well as to define the 8 -HPODE concentration that gives maximal radicalintensity, spins were quantified by $\mathrm{cw} X$-band spectroscopy. To avoid the pitfalls associated with absolute quantitation, most of the samples were quantified at $20 \mathrm{~K}$ utilizing $\mathrm{g}_{2}=2.25$ of the lowspin heme as internal standard. Since the reaction generating the tyrosyl radical as well as the mutation of Tyr327Phe is unlikely to influence this feature, normalizing the radical intensity to this value seems to be a valid approach. The results indicated that, although generated by manual mixing, the radical can be trapped reproducible and to the same extent in X-band and Qband tubes. In contrast, the radical-kinetics and enzyme activation was found to be sensitive to the utilized concentration of peroxide and 160-fold excess of 8-HPODE was identified as the peroxide concentration that enabled to generate the highest radical amount. While radical trapping was reproducible for a given enzyme batch, different batches showed a varying response towards peroxide treatment with radical intensities varying by as much as 4 -fold. In an attempt to absolutely quantify the amount of radical, radical intensities were measured at $110 \mathrm{~K}$ 
and quantified with a $100 \mu \mathrm{M}$ 4-Amino-2,2,6,6-tetramethylpiperidine-1-oxyl standard. These data suggest that, depending on the enzyme preparation, roughly between 0.1 and 0.4 spins per trimer were yielded. Considering the errors associated with determination of protein concentrations, quantification of radicals and the error propagation of both, this value is in reasonable agreement with the 0.6 spins per trimer reported by Fielding et al. (Fielding et al., 2011). For DEER-measurements the enzyme preparations yielding most radical upon activation were deployed.

Dipolar evolutions were recorded on samples prepared by mixing $100 \mu \mathrm{M}$ of the respective PpoA-variant with $16 \mathrm{mM}$ 8-HPODE in HEPES-buffer containing $30 \%$ glycerol and trapping the generated radical by freezing in liquid nitrogen after 4 to 6 seconds. The data were collected at 5 $\mathrm{K}$. For the measurement, the microwave frequency was set to the value maximally absorbed in the resonator and the magnetic field was adjusted to match the low-field transition of the $\beta$ proton split tyrosyl-radical. The pump pulse was applied $50 \mathrm{MHz}$ away on the tyrosyl radical's high-field line. The pulse length enabling the most efficient spin-inversion at the pump frequency was determined by a nutation experiment and found to be $\sim 50 \mathrm{~ns}$. The parameters of the pulsesequence at the observer frequency were set as follows: $\pi / 2=20 \mathrm{~ns} ; \tau_{1}=150 \mathrm{~ns} ; \tau_{2}=3200 \mathrm{~ns}$ and the shot repetition time was set to $100 \mathrm{~ms}$. The signal was averaged for $20 \mathrm{~h}$ and distances were extracted from data obtained in the time domain by Tikhonov regularization as implemented in DeerAnalysis2011 (G. Jeschke et al., 2006). The distances extracted from dipolar evolutions recorded on wild type samples resembled the distance-distribution that was already measured at X-band (Fielding et al., 2011): One major distance of $5.2 \mathrm{~nm}$ is accompanied by a minor distance of $4.1 \mathrm{~nm}$ (Figure $33 \mathrm{~A}+\mathrm{B}$ ). While the major distance is in good agreement with the equilateral distance triangle putatively spanned between the Tyr374s, the existence of a second distance is not in agreement with a solely paramagnetic center within a symmetric trimer. To assess whether this smaller distance might arise from an alternative radical-site, dipolar evolutions were recorded additionally on PpoA's Tyr327Phe-variant. According to the predicted structure of the DOX-active site (Section 4.3.1.1), this residue is the most likely candidate for formation of an additional radical-site. Interestingly, the obtained dipolar evolution (Figure $33 \mathrm{C}$ ) as well as the extracted distances (Figure $33 \mathrm{D}$ ) showed no significant difference to wild type enzyme, which might point out that Tyr327 is not a major alternative radical-site and, at the same time, strengthens the hypothesis of Tyr374 being the catalytic competent radical-site. Considering the second hypothesis according to which Tyr327 serves as hydrogen-bond donor to stabilize the catalytic active Tyr374, one would expect to see a broader distance distribution in the Tyr327Phe-variant. Although no broadening was extracted from the measured dipolar evolutions for this variant, this does not necessarily contradict this hypothesis. The low signal-tonoise ratio and the rather short dipolar evolution time prevent a reliable extraction of distance distributions from the measured data. 

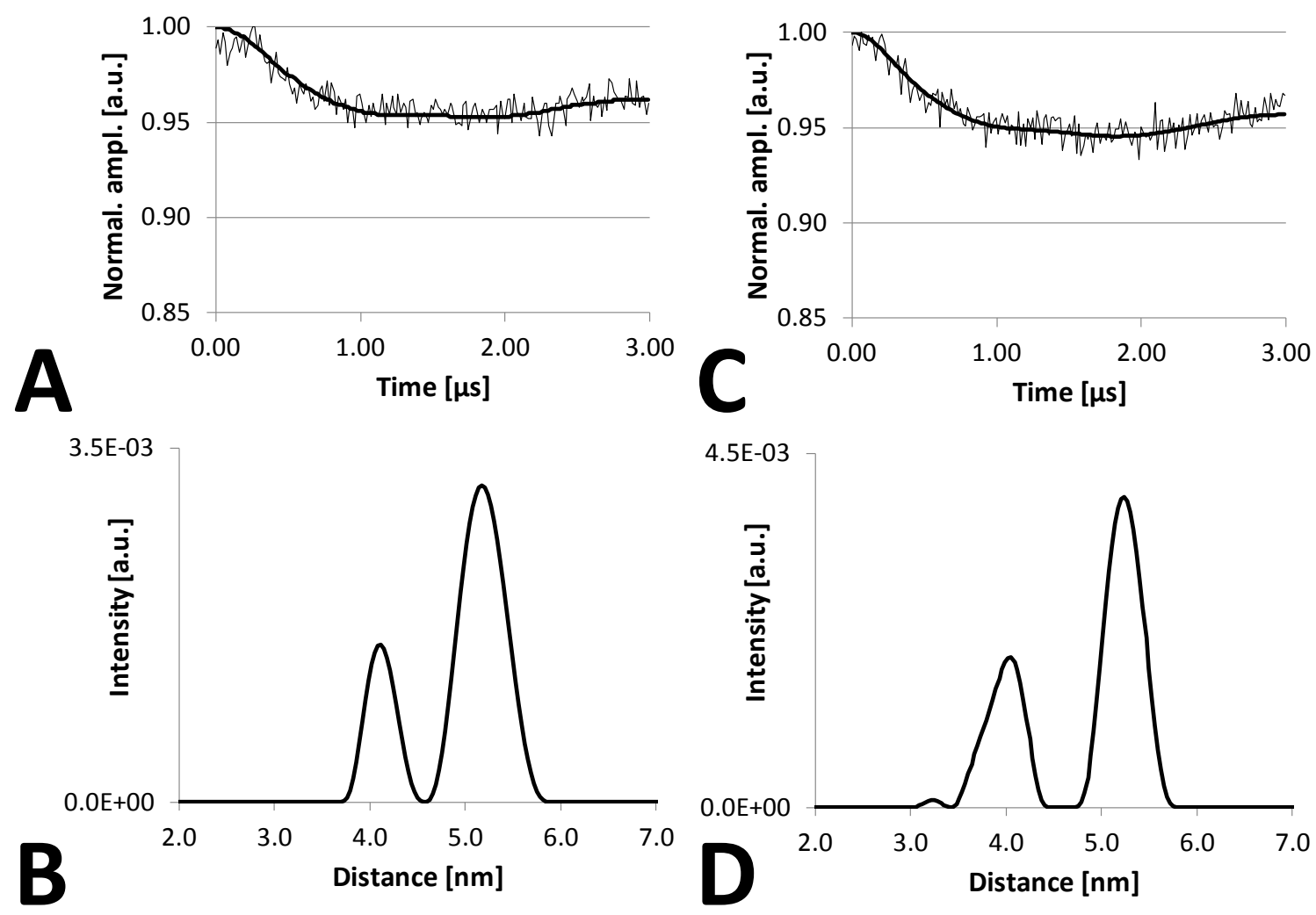

Figure 33: Dipolar evolutions and distance distributions of tyrosyl-radical distances in PpoA. A Background

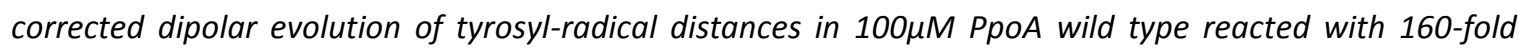
excess 8-HPODE and aged for $6 \mathrm{~ms}$. The trace was accumulated at $5 \mathrm{~K}$ and averaged for $20 \mathrm{~h}$. Experimental details are given in the text. The here shown evolution is consistent with more than 5 independently measured ones. B Distance distributions extracted from A. C Background corrected dipolar evolution of tyrosyl-radical distances in 100 M PpoA Tyr327Phe. The experimental procedure yielding the radical is identical to the one specified for $A$ and a consistent trace was obtained in an independent measurement. $\boldsymbol{D}$ Distance distributions extracted from $C$.

Although deployment of the respective variant revealed that the $4.1 \mathrm{~nm}$ distance does not stem from an additional radical formed at Tyr327, no alternative hypothesis where it might arise from was derived so far. Considering that PpoA is an enzyme that is characterized by the presence of three paramagnetic centers in the active state (i.e. tyrosyl radical; low-spin heme and high-spin heme) (Fielding et al., 2011), it is reasonable to assume that the minor distance might arise from dipolar couplings of the tyrosyl to one of the hemes. Based on the fact that inversion of high-spin heme magnetization requires different energies than applied and the proposal that the DOXheme (i.e. the high-spin heme) is in the ferryl state in the activated enzyme and thus is EPRinactive, one can reason that dipolar couplings to the low-spin heme might be picked up. Interestingly, the distances between the proposed tyrosyl radical-site and the low-spin heme within the low-resolution SAXS-model are in agreement with the measured distance distribution (Figure $34 \mathrm{~A}$ ). While the distances between the low-spin hemes of each monomer and one 
tyrosyl-heme distance are too large to be observed (i.e. $d>8 \mathrm{~nm}$ ), the remaining two tyrosylheme distances are in the same order of magnitude as the Tyr374* distances and a bit smaller, respectively, and thus could explain the measured distance distribution. To test this hypothesis, a PpoA-variant with a reduced low-spin heme-content (Phe799Leu; Section 4.4.3) was measured (Figure $34 \mathrm{~B}$ ). Albeit the distance distribution extracted from the dipolar evolution of this variant (Figure $34 \mathrm{C}$ ) indicates that the smaller distance is gone in the absence of low-spin heme, an unequivocal statement linking this distance to a tyrosyl P450-heme distance can't be made, because a DEER-experiment placing the pump pulse $60 \mathrm{MHz}$ away at the low-field side of the tyrosyl (i.e. in better resonance to the low-spin heme) did not result in a dipolar evolution with a modulation depth significant enough to be analyzed (data not shown).

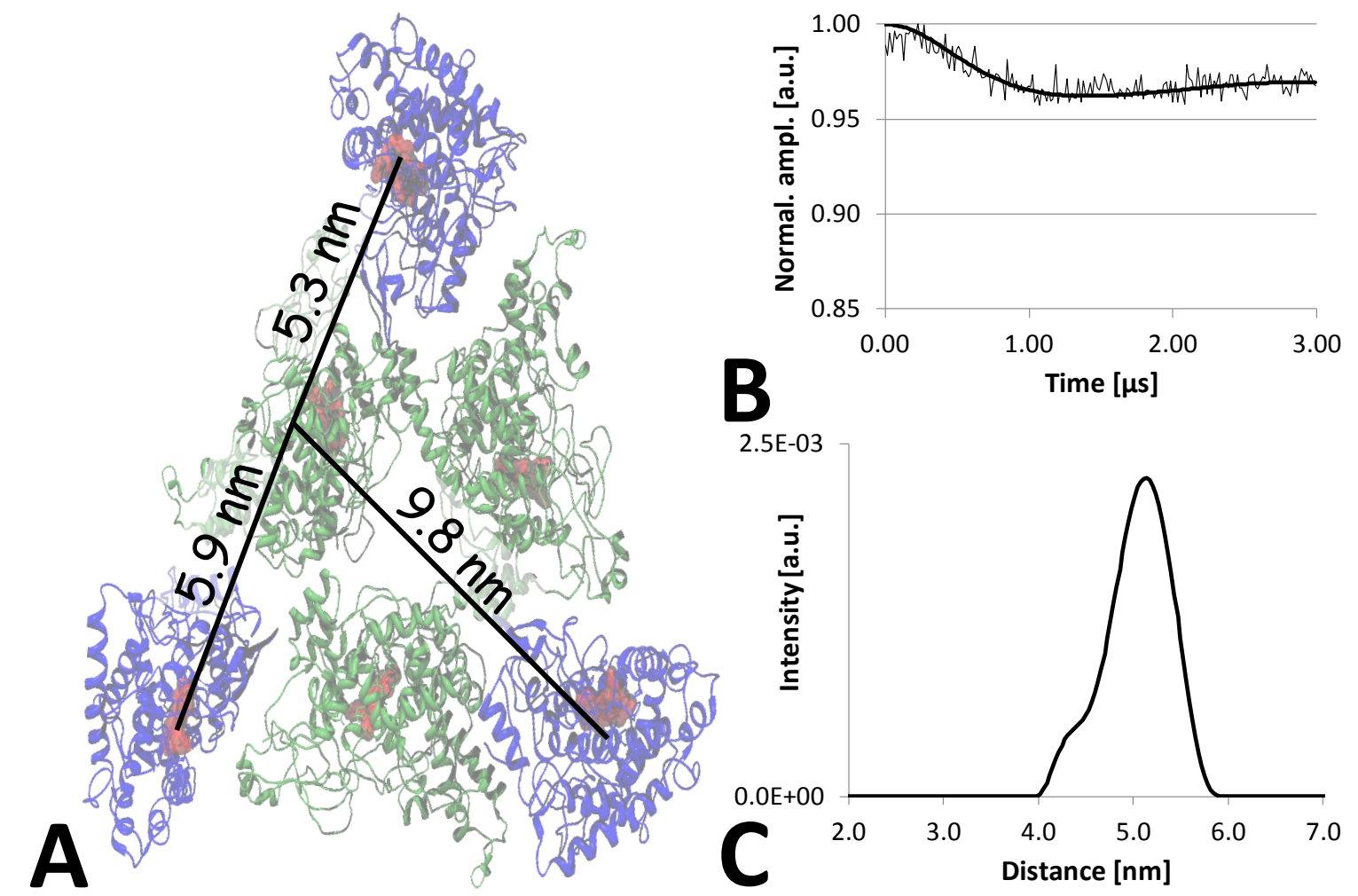

Figure 34: The minor distance observed in PpoA might be caused by dipolar coupling of the catalytic competent tyrosyl to the low-spin heme. A Expected distances of the low-spin hemes to one of the proposed tyrosyl-radical sites (Tyr374). Remarkably, one of the expected distances has the same magnitude as the tyrosyl-tyrosyl distances, one is slightly shorter and the third is too large to be detected by DEER. Note that, due to symmetry, the distances between the other tyrosyls and the low-spin hemes will be identical. B Background corrected dipolar evolution of tyrosyl radical distances in $100 \mu M$ PpoA_Phe799Leu reacted with 160-fold excess of 8-HPODE and aged for $6 \mathrm{~ms}$. The trace was accumulated at $5 \mathrm{~K}$ and averaged for $20 \mathrm{~h}$. Experimental details are given in the text. The shown evolution is consistent with the dipolar evolution obtained for the PpoA_His1004Ala variant, which also has a reduced heme occupancy ((Brodhun et al., 2009); Dr. Alistair Fielding, personal communication) C Distance distributions extracted from $B$. 


\subsection{Kinetic isotope effects to probe rate-limiting steps of hydrogen abstraction}

\subsubsection{UTILIZED PROBES}

To assess the contribution of hydrogen abstraction steps in the distinct PpoA-domains on the overall enzyme kinetics, Dr. Andre Nadler (Institute for Organic and Biomolecular Chemistry, University Goettingen) synthesized regio-specifically dideuterated oleic acids. One substrate was dideuterated at $C_{8}$ (8,8-Dideutero oleic acid, $\left.C_{8}-d_{2}-O A\right)$, while another had these labels on $C_{5}(5,5-$ Dideutero oleic acid, $\mathrm{C}_{5}-\mathrm{d}_{2}-\mathrm{OA}$ ) (Figure 35 ). Therefore the latter one served as probe to test the kinetic contribution of $C_{5}$-hydroxylation on the overall reaction, while $C_{8}-d_{2}-O A$ was utilized to probe the kinetic significance of the hydrogen abstraction step preceding the insertion of molecular oxygen. Or, to give it in other words, $\mathrm{C}_{8}-\mathrm{d}_{2}-\mathrm{OA}$ can give information on the kinetic contribution of the reaction taking place in the DOX-domain of the enzyme and the kinetics of $\mathrm{C}_{5}-\mathrm{d}_{2}-\mathrm{OA}$ conversion will provide knowledge about the kinetic relevance of fatty acid hydroperoxide rearrangement taking place in the cytochrome P450-domain of the enzyme.

\subsubsection{PSEUdo Steady-STATE KINetics}

The reaction kinetics was continuously assayed by monitoring the insertion of molecular oxygen into the fatty acid. Since oxygen is inserted in equimolar amounts into the substrate, this oxygen consumption can be directly related to enzyme kinetics. Figure 36 shows representative traces of this oxygen depletion for $100 \mu \mathrm{M}$ of the respective substrates. In figure $36 \mathrm{~A}$ the proposed mechanistic homology to PGHS is illustrated by the sigmoidal shape of the obtained oxygen consumption curves, reflecting enzyme activation and inactivation processes. On a longer timescale this is also evident for $\mathrm{C}_{8}-\mathrm{d}_{2}-\mathrm{OA}$ (Figure $36 \mathrm{~B}$ ). The first derivatives of these curves reveal unequivocally that the entire kinetics is governed by these opposite effects and that a phase of highest activity is reached only transiently (Figure $36 \mathrm{C}$ ). This dependence on activation and inactivation processes permits the application of conventional steady-state theory for evaluation of the observed kinetics and complicates the quantitative interpretation of the results. Since the intention of the here described experiments was to obtain data on the rate limiting steps under physiological conditions and a comprehensive investigation of the enzyme with all its characteristics, including activation- and inactivation-phases, a pre-activation of the enzyme with any kind of peroxide was not conducted. 

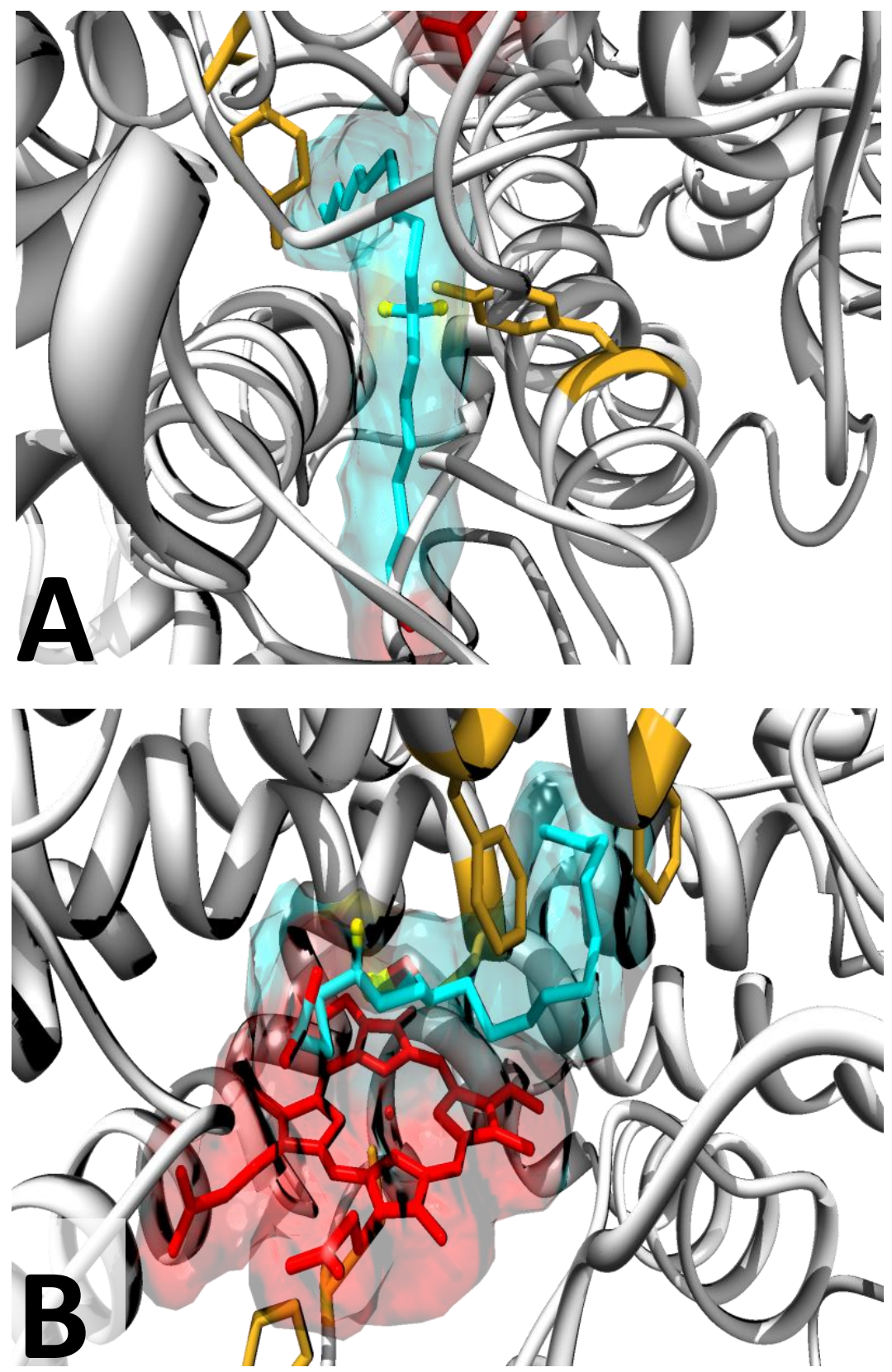

Figure 35: Probes utilized to determine the kinetic contribution of hydrogen transfer steps in the DOXdomain (A) and the P450domain (B) of PpoA. A Within the DOX-domain hydrogen is abstracted from oleic acid's $\mathrm{C}_{8}$ and transferred to the catalytic active tyrosine (which is most likely Tyr374, the left tyrosine in this figure; the right one is Tyr327). By specific dideuteration of this carbon, the corresponding reaction rate is diminished. $\boldsymbol{B}$ Within PpoA's P450-domain, the N-terminally formed 8HPOME is rearranged to $5,8-$ DiHOME. Therefore, a hydrogen/deuterium has to be abstracted, most likely by compound I, from substrate's $C_{5}$. Substrates are rendered in cyan with oxygen atoms in red and deuterium in yellow.

To overcome the difficulties associated with the description of the obtained biexponential kinetics, the transiently reached point of maximal velocity was extracted from each trace for the different substrate concentrations. The dependency of these values from substrate concentration was pragmatically described by a hyperbolic equation similar to the one, known from Michaelis-Menten-kinetics (Figure 37). Nevertheless, one should keep in mind that no steady state was reached and thus the parameters of this hyperbolic equation do not have the meaning of Michaelis-Menten parameters. Yet, the obtained parameters might be utilized to quantify the KIEs observed for the distinct isotopologues (Table 4). While the magnitudes of the measured data indicate that hydrogen abstraction from $\mathrm{C}_{8}$ is the major rate-limiting step of the reaction, the influence of $\mathrm{C}_{5}$-dideuteration on oxygen depletion kinetics indicates that the two enzyme domains are not acting independent from each other. 


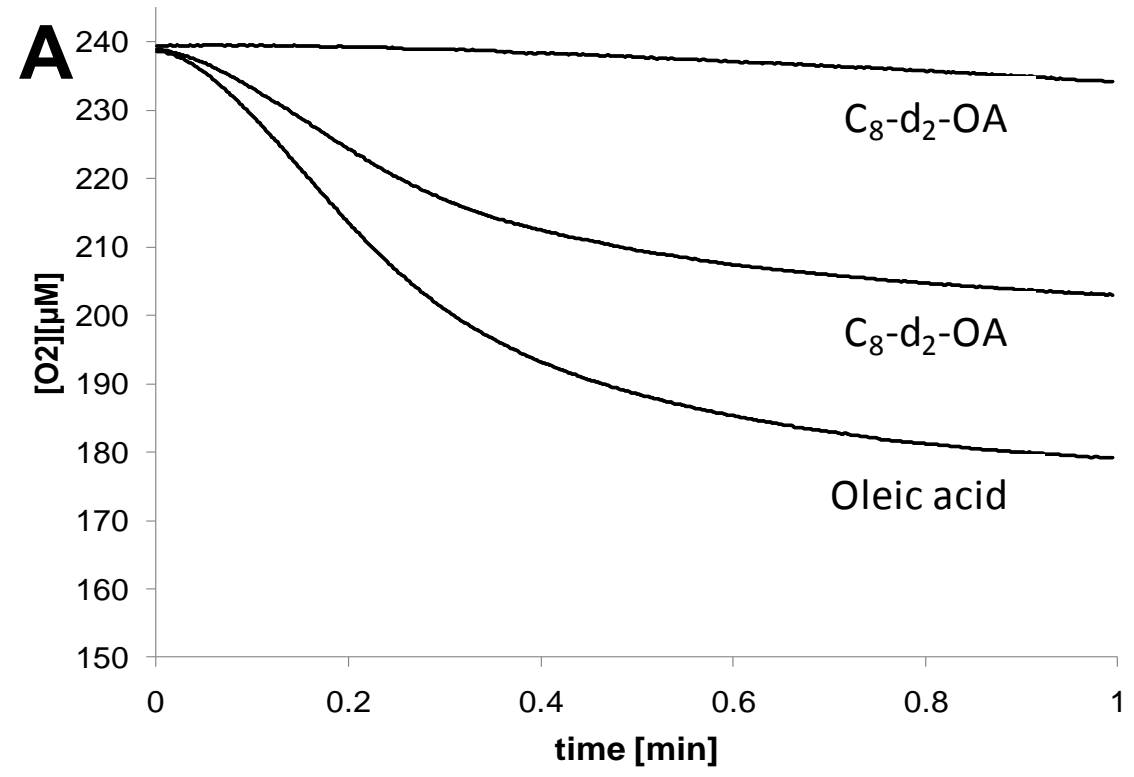

Figure 36:

representative

oxygen

consumption

kinetics, obtained

for conversion of

500 nM PpoA with

$100 \mu \mathrm{M}$ of various

substrates.

A Oxygen

depletion kinetics

obtained for

conversion of oleic

acid, $C_{5}-d_{2}-O A$ and

$C_{8}-d_{2}-O A$,

respectively.

Shown are

representative

traces from three

independent

measurements.

B Oxygen

depletion kinetics

obtained for

conversion of $\mathrm{C}_{8}$

$d_{2}-O A$.

C First derivatives

of the kinetic

traces from $A$.

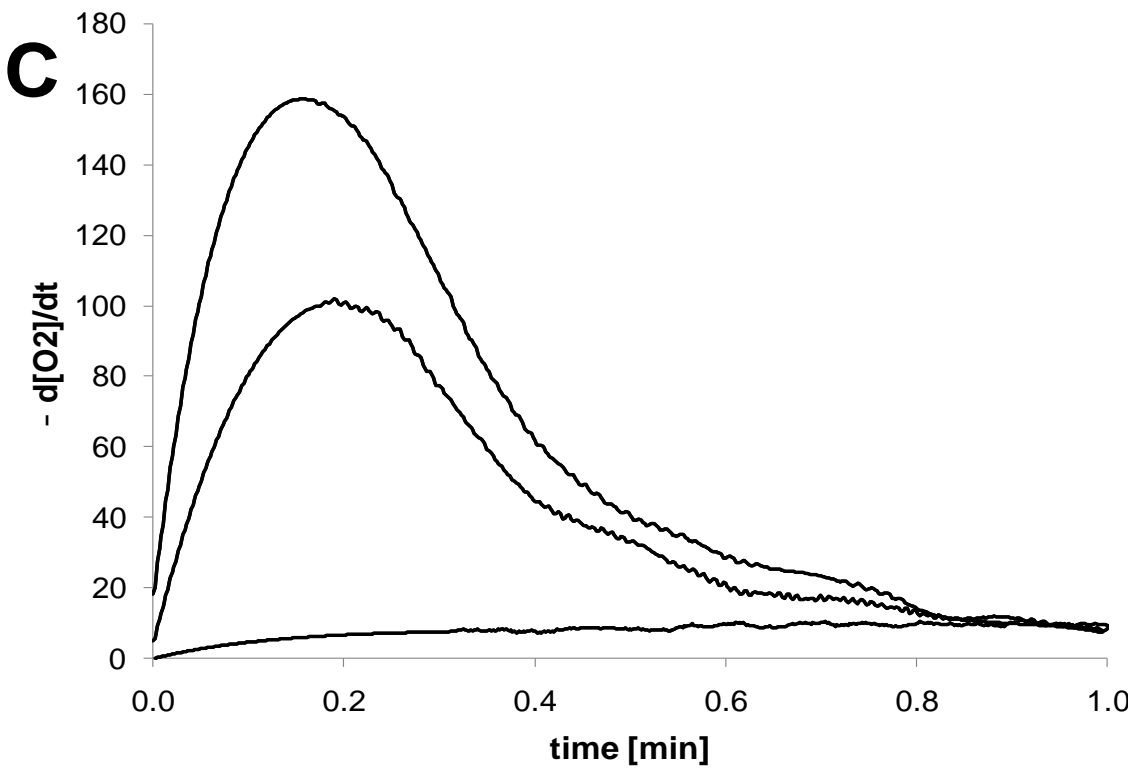




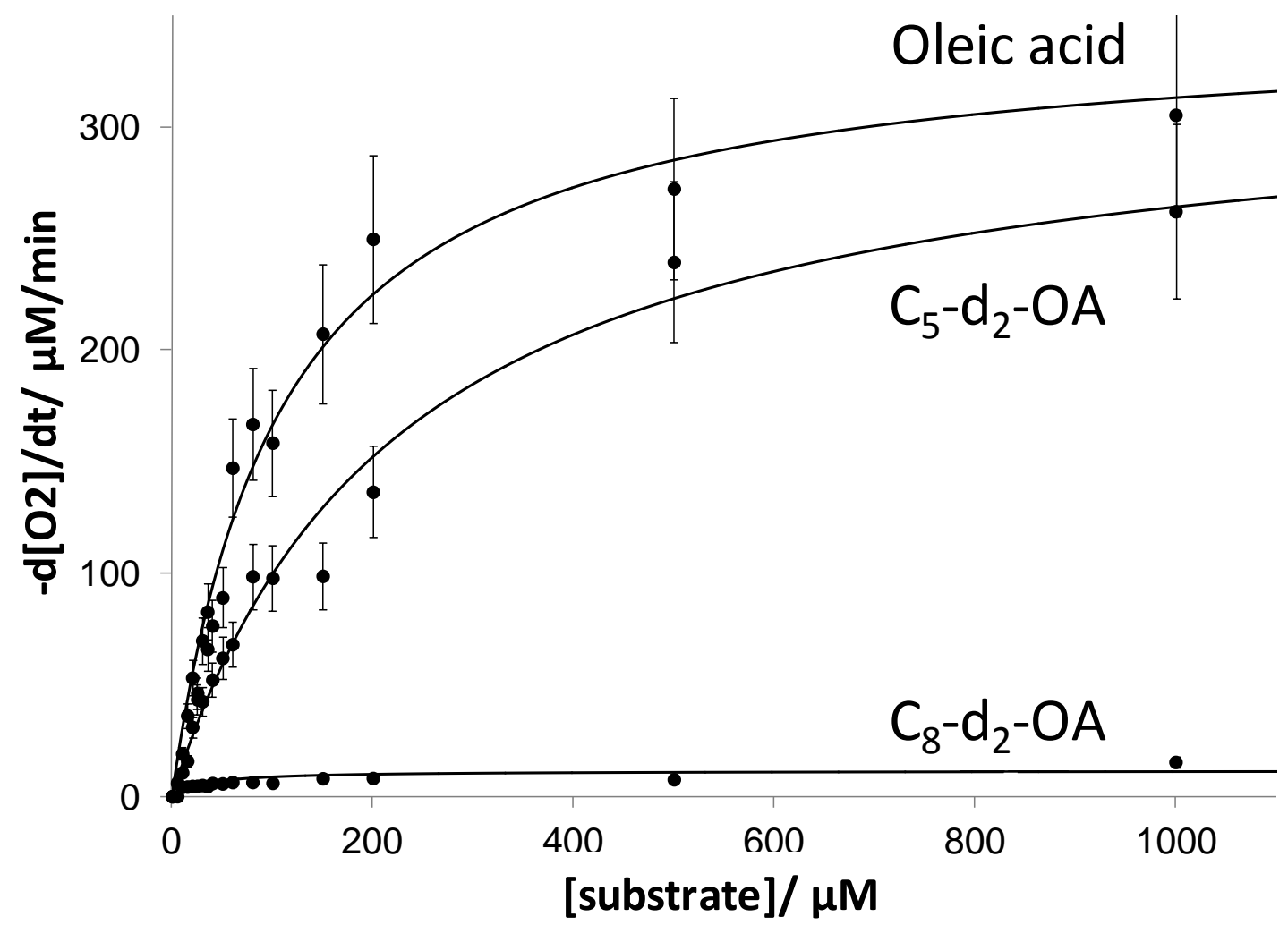

Figure 37: Pseudo-Michaelis-Menten kinetics obtained for conversion of oleic acid (upper trace); $C_{5}-d_{2}-O A$ (middle trace) and $\mathrm{C}_{8}-\mathrm{d}_{2}-\mathrm{OA}$ (lower trace) by PpoA. By measurement of fifteen distinct substrate concentrations as triplicates, the coefficient of variation was found to be up to $20 \%$ for this method. To illustrate the significance of the measurements, this coefficient of variation is shown for each substrate concentration measured.

\begin{tabular}{ccc}
\hline & \multicolumn{3}{c}{ Substrate } \\
\hline Kinetic parameter & $\mathrm{C}_{5}-\mathbf{d}_{\mathbf{2}}-\mathrm{OA}$ & $\mathrm{C}_{\mathbf{8}}-\mathrm{d}_{\mathbf{2}}-\mathrm{OA}$ \\
\hline $\mathrm{V}_{\mathrm{H}} / \mathrm{v}_{\mathrm{D}}$ & 1,1 & 33,4 \\
\hline$(\mathrm{v} / \mathrm{k})_{\mathrm{H}} /(\mathrm{v} / \mathrm{k})_{\mathrm{D}}$ & 2,2 & 9,6 \\
\hline
\end{tabular}

Table 4: Deuterium KIEs for conversion of oleic acid and its $C_{5}$ - $d_{2}$-and $C_{8}-d_{2}$-derivatives, respectively. Note that the kinetics do not obey the Michaelis-Menten equation and thus interpretation of the obtained KIEs is not straightforward. 


\subsubsection{END-POINT MEASUREMENTS}

To probe, whether the results obtained for oxygen depletion are reflected in the amount of product formed, conversions of $100 \mu \mathrm{M}$ of the respective substrate by PpoA were quenched after 2 min incubation time and extracted with $\mathrm{Et}_{2} \mathrm{O}$. Although the HPLC results (Figure 38) correlated with the results of oxygen consumption and showed roughly the same amount of activity reduction for the distinct substrates, LC-MS ${ }^{2}$ analysis revealed that there was a significant change in the relative amount of byproducts of the reaction (Figure 39). While for conversion with oleic acid and $\mathrm{C}_{8}-\mathrm{d}_{2}-\mathrm{OA}$ the product pattern showed the known distribution with 6,8-DiHOME and 9,10-epoxy-8-hydroxy-octadecanoic acid as side products, the amount of these minor products was doubled for the substrate dideuterated at $C_{5}$. Concomitant with this increase, the amount of the main product was decreased by the same extent.

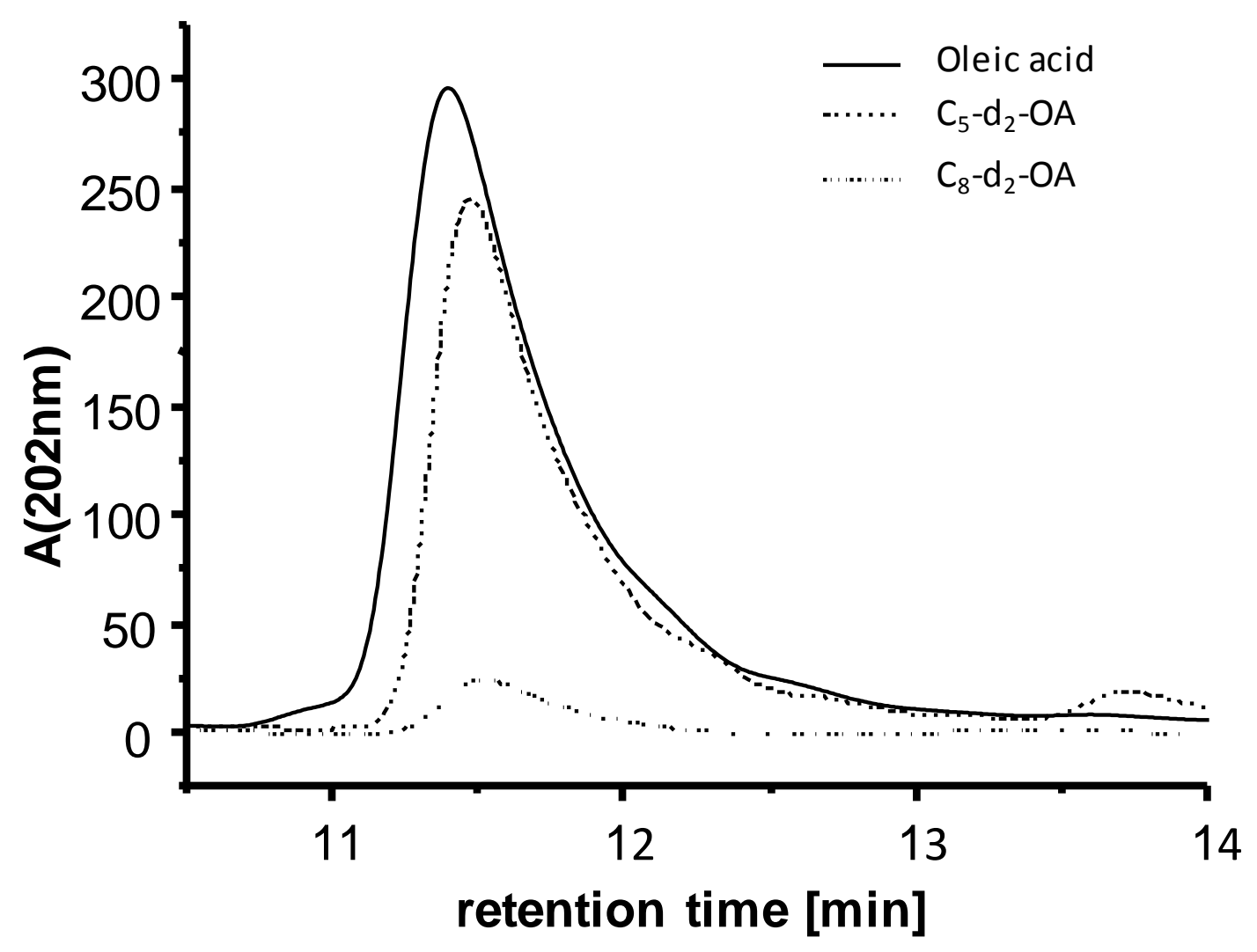

Figure 38: Quantitative analysis of the main product (5,8-DiHOME) formed upon conversion of PpoA with differentially dideuterated oleic acid. Shown are UV-signals of the product-pattern separated by RP-HPLC. Retention time of the main product was verified by authentic standard. Shown are representative chromatograms of three independent conversions 


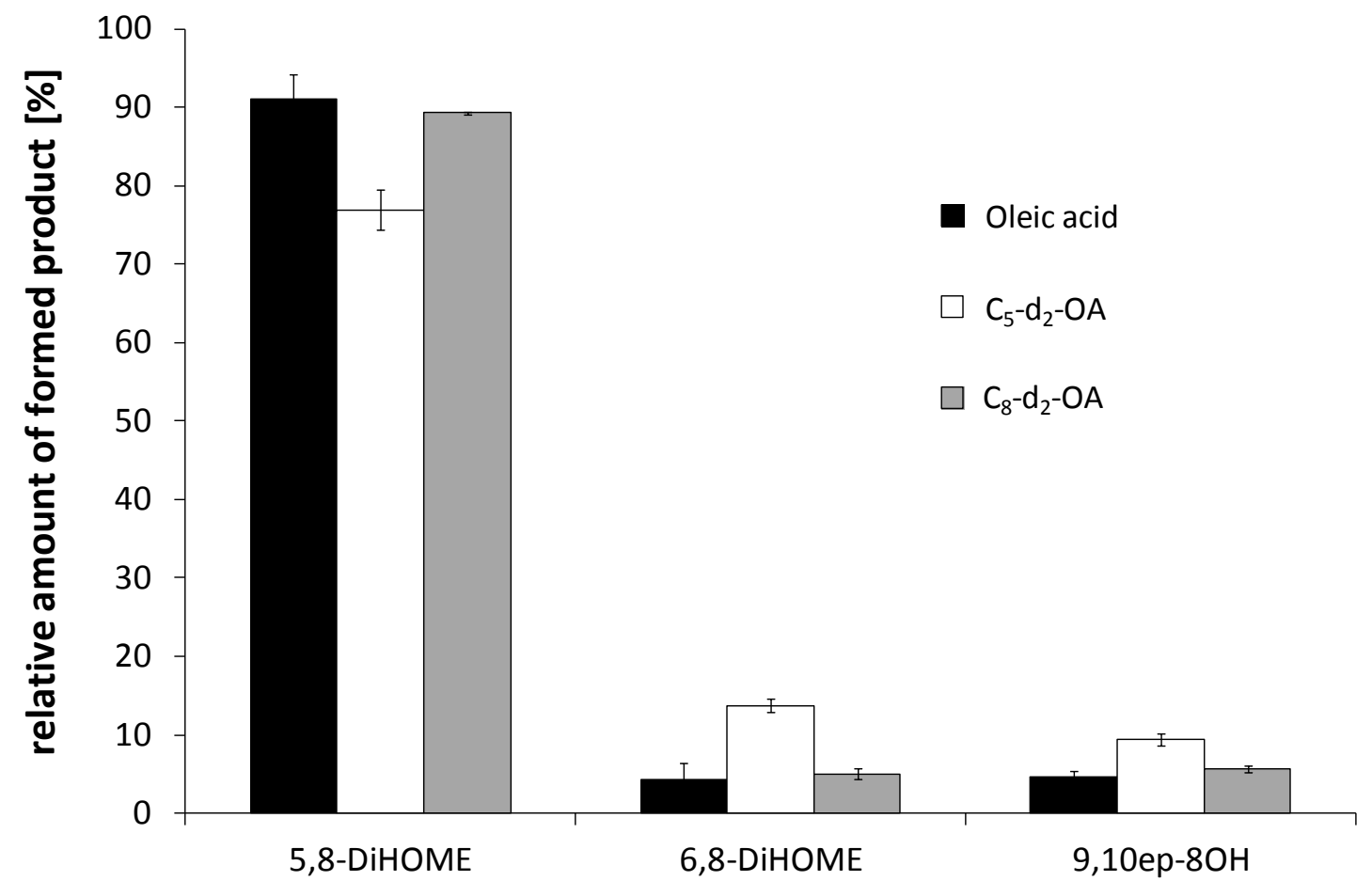

Figure 39: $L C / M S^{2}$-analysis of products formed upon conversion of 100 $\mu \mathrm{M}$ differentially dideuterated oleic acid by 500nM PpoA. The reaction was accomplished for two minutes and products were subsequently extracted with $E t_{2} O$. Formed products were identified by $L C-M S^{2}$ (as described in Section 3.7) and quantified by LC-MS. Shown are the arithmetic mean as well as the corresponding standard deviation for results from three independent conversions. 9,10ep-80H is short for 8-hydroxy-9,10-epoxy-octadecanoic acid.

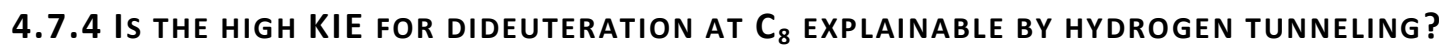

The most straightforward way to address this question is to measure the temperature dependency of the reaction. Hampering this approach, activity is beyond the systems limit of detection for the substrate $\mathrm{C}_{8}-\mathrm{d}_{2}-\mathrm{OA}$ at temperatures below $8{ }^{\circ} \mathrm{C}$. Additionally, the activity shows a significant drop at $40{ }^{\circ} \mathrm{C}$ indicating that enzyme denaturation already occurs at this temperature. Constrained by these two limits, the temperature range that can be covered is rather small and thus the results deduced from these measurements should be treated with care. Nevertheless, the measured temperature dependency within this limited range revealed no striking difference in the Arrhenius prefactors for the reaction of PpoA with oleic acid or $\mathrm{C}_{8^{-}}$ $d_{2}-\mathrm{OA}$ and thus contradicts the possibility of hydrogen tunneling (data not shown). 


\subsubsection{EXPLAining THE EFFECT OF Dideuteration AT $C_{5}$ ON OXYGEN CONSUMPTION}

The most likely explanation for the observed influence of dideuteration at $C_{5}$ on dioxygenation rate would be the existence of an inter-domain communication. One possible mechanism of this inter-domain communication could be intermediate-channeling from the $\mathrm{N}$-terminal domain to the P450-domain of the enzyme. In principle, there are at least three ways of intermediate channeling:

(i) A closed hydrophobic tunnel for intermediate transfer (Cheng et al., 2008)

(ii) Electrostatic channeling of the intermediate across the protein surface (Cheng et al., 2008)

(iii) 'Channeling' by proximity of the active sites, resulting in a microenvironment with locally higher intermediate concentrations (Bauler et al., 2010)

Due to the chemical properties of the intermediate yielded in the reaction of PpoA with oleic acid, possibility (ii) seems to be unlikely. If substrate channeling occurs within a closed hydrophobic tunnel, one would expect that in a competitive approach using labeled fatty acid (50 $\mu \mathrm{M}{ }^{13} \mathrm{C}_{18}$-linoleic acid) and unlabelled intermediate (50 $\mu \mathrm{M}$ 8-HPODE) the majority of end product would be labeled (Spivey and Ovádi, 1999). As the result of this isotope dilution in figure 40 demonstrates, this is not the case for PpoA. Contrary an accumulation of labeled intermediate was observed and most of the end product formed was unlabeled, indicating that the P450-domain of the enzyme can use free intermediate as substrate for the rearrangement and that the hydroperoxide product of the DOX-domain is released to the buffer. These results could point out that the 'channel' between the both domains might be defined by not more than steric adjacency resulting in a direct equilibration of the intermediate between both active sites, which might be a kinetic advantage for the overall reaction to yield the desired end product. However, the macromolecular structure as it is supported by the SAXS-data (Section 4.6.1) does not substantiate this idea. Contrary, the proposed active sites (Section 4.3) and substrate access routes are not facing each other in this model. 


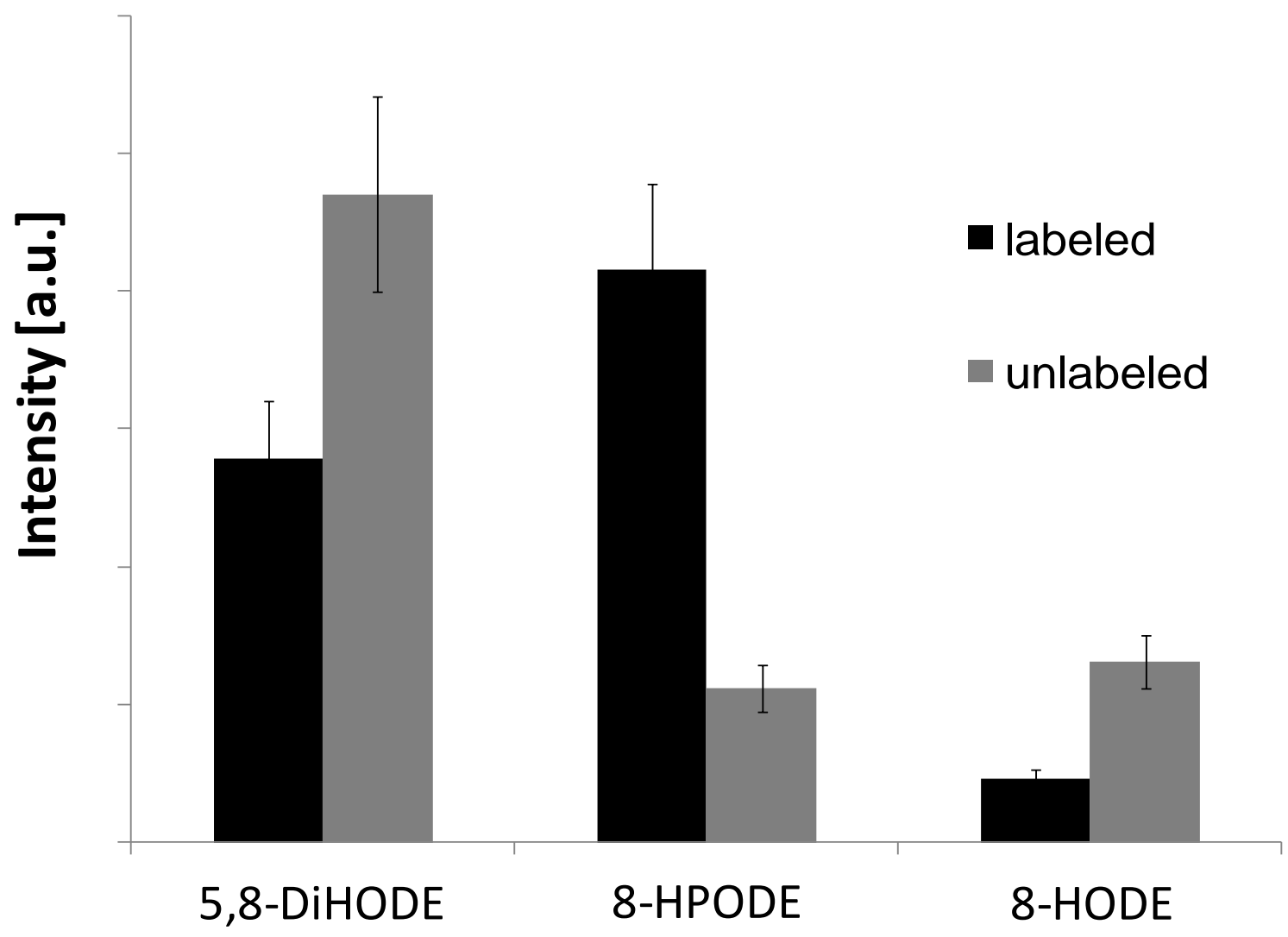

Figure 40: Isotope dilution experiment for the reaction of PpoA with labeled linoleic acid and unlabeled intermediate (8-HPODE) suggests a lack of a hydrophobic tunnel between both domains. 50uM of each substrate were reacted with $0.1 \mu \mathrm{M}$ PpoA. The products formed after $45 \mathrm{sec}$ were extracted and quantified by LC/MS. Shown are the arithmetic mean as well as the corresponding standard deviation for products quantified from three independent conversions.

Another hypothesis that could explain the observed influence of $\mathrm{C}_{5}$-dideuteration on the kinetics of dioxygen consumption is that the enzyme exhibits some kind of allosteric regulation or a halfof-site reactivity as it was reported e.g. for PGHS (Yuan et al., 2006; Dong et al., 2011). As already stated in the introduction, this enzyme is a dimer, but interestingly, upon substrate binding to one subunit, a conformational change will inactivate the other one and hence only one of both subunits is active at the same time. A similar mechanism occurring between the distinct domains of PpoA and thus a conformational change occurring upon substrate binding to one domain and preventing substrate binding to or conversion by the other domain could also explain the observed effect. The reduced kinetic rate of fatty acid hydroperoxide rearrangement caused by dideuteration at $\mathrm{C}_{5}$ would consequently also affect the activity of the fatty acid hydroperoxide formation in PpoA's DOX-domain. If this hypothesis is true, one would expect that the enzyme's oxygen consumption rate depends on whether there is additional fatty acid hydroperoxide present in the buffer. Without the initial presence of this substrate of the P450-domain, the dioxygen insertion should proceed with a faster rate. Contrary, if substrate for the cytochrome P450 catalyzed hydroperoxide rearrangement is present, the conformational change caused by 
this reaction should slow down the dioxygenation rate. Nevertheless, one has to consider that 8HPODE does not only serve as substrate for PpoA's P450-domain, but also activates the DOXdomain by oxidation of the heme. As an opposite effect, this enhanced activation might actually mask a potential allosteric regulation. However, the results of the corresponding competitive experiment, deploying $100 \mu \mathrm{M}$ of the substrate of the DOX-domain and a varying concentration of the P450-substrate, show a rather severe dependence of the DOX-activity on the substrateconcentration available for the rearrangement-reaction, which might be interpreted as first proof for an allosteric regulation (Figure 41).

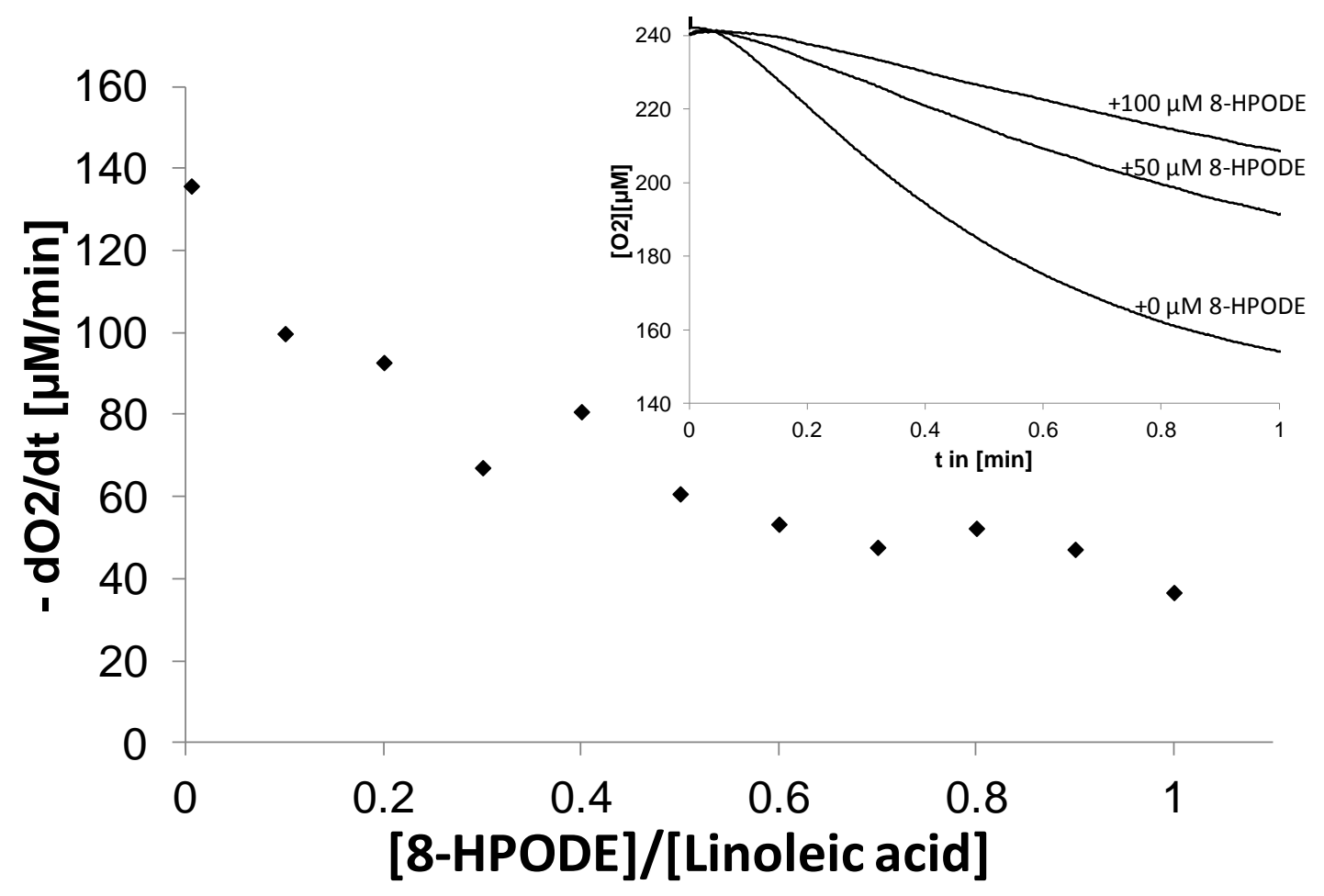

Figure 41: Decrease of dioxygenation velocity, due to increase of the initially present intermediate concentration, might be interpreted as hint for a half-of-site reactivity of both PpoA domains. Shown is the quantitative determination of maximal DOX-activity in dependence of substrate concentration available for the P450-reaction. 0.5 MM PpoA was reacted with $100 \mu \mathrm{M}$ linoleic acid and a varying concentration of 8HPODE. For each ratio [8-HPODE]/[Linoleic acid] a single measurement was performed. Inset: Oxygen consumption kinetics obtained for PpoA catalyzed conversion of $100 \mu \mathrm{M}$ linoleic acid without, with $50 \mu \mathrm{M}$ and with $100 \mu M$ 8-HPODE reveals a significant dependency of DOX turn-over on substrate concentration available for the rearrangement reaction catalyzed by the P450-domain. 


\subsubsection{Stereochemistry of the dioxygenation, catalyzed by PpoA}

The stereochemistry of the sequential oxygenation yielding dihydroxy fatty acids was elucidated for the homologue enzyme 7,8-LDS from Gaeumannomyces graminis (M Hamberg et al., 1994). This enzyme abstracts the pro-S hydrogen from $\mathrm{C}_{8}$ and inserts oxygen antarafacially. The following conversion of $8 R$-HPODE to a vicinal diol in threo configuration takes place suprafacially. To establish whether also PpoA abstracts the pro $S$ hydrogen of the fatty acid substrate's $\mathrm{C}_{8}$, linoleic acid enriched to $64 \%$ with an $8 R$-deuterium label was utilized. Calculated from the natural abundance of ${ }^{13} \mathrm{C}(1.1 \%)$, one would expect approx. $20 \%$ of end product to be one Da heavier than the nominal mass, assuming that the compound consists of 18 carbons. For the conversion of linoleic acid by PpoA, this expectation value is quite exactly met by the measured value (Table 5). As a result of conversion of linoleic acid enriched with $8 R$-Deuterolinoleic acid one would mathematically expect $58 \%$ of the heavier isotopologue, if the deuterium label is retained. This theoretical value is the sum of the heavier ${ }^{13} \mathrm{C}$-isotopologue (i.e. the isotopologue with one atom ${ }^{13} \mathrm{C}$ ) derived from $36 \%$ linoleic acid and the light ${ }^{13} \mathrm{C}$ isotopologue (i.e. the isotopologue without any ${ }^{13} \mathrm{C}$ ) derived from the deuterium-labeled linoleic acid $(0.2 * 0.36+0.8 * 0.64)$. If one furthermore considers the isotopologues with an additionally by one Da increased molecular mass, the theoretically expected distribution can be derived as described and would be $29 \%, 58 \%$ and $13 \%$, for 311,312 and $313 \mathrm{Da}$, respectively. Evaluation of the experimental data for all three masses reveals that the measured values do not fit as perfect as outlined in table 5. However, the measured relative distribution: $36 \%, 53 \%$ and $11 \%$ is still close to the expected distribution. Especially when considering the mass uncertainty of the mass spectrometer used, the here reported deviation from theory is negligible. Since the experimental observed values are in agreement with the theoretically derived ones, one can deduce that the pro $S$ hydrogen is abstracted from $C_{8}$ and not the pro $R$ hydrogen. Hence, at least the first step of the reaction-mechanism has the same stereochemistry as the homologue reaction catalyzed by 7,8-LDS from $G$. graminis.

\begin{tabular}{ccc}
\hline Conversion of & Area 5,8-DiHODE $/ \mathrm{m} / \mathbf{z}=\mathbf{3 1 1}$ & Area 5,8-DiHODE $/ \mathrm{m} / \mathbf{z}=\mathbf{3 1 2}$ \\
\hline Linoleic acid & $84 \%$ & $16 \%$ \\
\hline $\begin{array}{c}\text { 64\% 8R-Deutero-linoleic acid/ } \\
\text { 36\% Linoleic acid }\end{array}$ & $43 \%$ & $57 \%$ \\
\hline
\end{tabular}

Table 5: Relative quantity of 5,8-DiHODE isotopologues formed upon conversion of PpoA with linoleic acid and linoleic acid enriched with its 8R-deutero isotopologue, respectively. The arithmetical expected nominal mass of the end product of linoleic acid conversion by PpoA is $311 \mathrm{Da}$. The amount of end product with this mass and its isotopologue with a one Da heavier molecular weight was quantified by LC-MS. 


\subsection{Protein film voltammetry of PpoA}

In order to assess the ability of PpoA to be investigated by protein-film voltammetry, a pyrolytic graphite electrode was coated with DDAB. This promoter/electrode-system is a suitable starting point to investigate the electrochemical behavior of a heme containing protein (Section 3.6).

However, immediately upon bringing the electrode prepared with a film of DDAB into the enzyme solution, a visible precipitate formed. Since this might indicate enzyme denaturation, the enzyme solution was checked for integrity of the native state by UV-Vis-spectroscopy and conversion of linoleic acid. Remarkably, both criteria showed characteristics deviating from the native enzyme and indicating enzyme denaturation. Hence, one has to keep in mind that the measured electrochemical properties will not reflect the physiological situation, but merely represent the artificial situation of the heme enzyme immobilized in a DDAB-film. The electrolyte

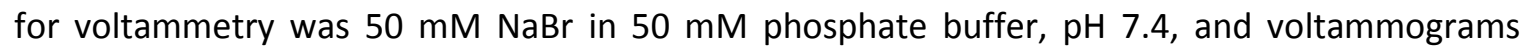
were measured against a silver reference-electrode. Both, the cyclic voltammograms as well as the square wave voltammograms (Figure 42) show a single midpoint potential, which might be caused by an unresolvable overlap of the midpoint potentials of both PpoA hemes or is indicative of a severe denaturation of rather one than both active sites. The value of this midpoint potential depends on the scan speed of the cyclic voltammograms and changes from $0.34 \mathrm{~V}$ (for a scan speed of $10 \mathrm{mV} / \mathrm{sec}$ ) to $-0.46 \mathrm{~V}$ (for a scan speed of $50 \mathrm{mV} / \mathrm{sec}$ ). Because this latter scan speed already leads to a severe perturbation of the voltammogram shape, it is reasonable to assume that acceptable midpoint potentials can be measured only for scan speeds of up to $30 \mathrm{mV} / \mathrm{sec}$. Interestingly, the value obtained for this scan speed $(-0.42 \mathrm{~V})$ is in perfect agreement with the value obtained from square wave voltammetry.
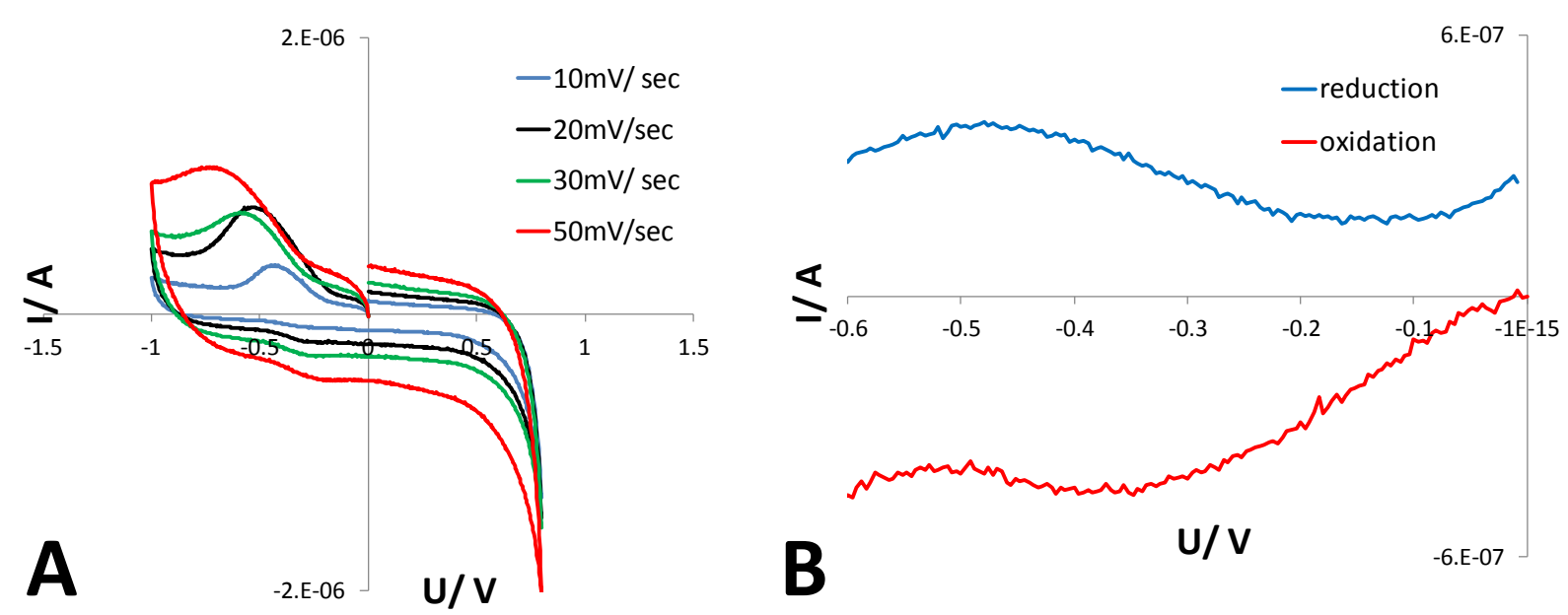

Figure 42: Protein film voltammetry of PpoA immobilized on a pyrolytic graphite electrode coated with $D D A B$. A Cyclic voltammograms obtained for different scan speeds. B Square wave voltammogram. Details of the experiment are specified in the experimental part of this thesis and the measured voltammograms are obtained from a single immobilization experiment. 
Results 


\section{DISCUSSION}

\subsection{Structure-Function-Relationship}

In order to establish a structure-function relationship for PpoA, it was attempted to crystallize the enzyme, obtain X-ray diffraction data and finally solve an atomic structure. Despite some efforts and the exploration of unusual techniques to eventually rescue the crystallization trials (Section 4.2), no diffraction quality crystals of PpoA were obtained. To derive structural information nonetheless, the single domains, which functionally resemble distinct oxylipin producing enzymes found in other species, were utilized for template-based structure prediction (Section 4.3). Evaluation of the predicted structures (Figures 15-17) was based on the fact that several aspects of structure-function-relationship, exemplary including substrate binding to heme-dioxygenases and mechanisms of peroxide-cleavage by atypical cytochrome P450's, have been elucidated in these homologue enzymes (Koszelak-Rosenblum et al., 2008; D.-S. Lee et al., 2008). However, one has to keep in mind that the predicted models will not correctly reflect all structural details and substrates might also bind with a slightly changed conformation. Exemplary the fatty acid bound in the DOX-domain might exhibit a different position as proposed and hence the geometry and distances between carbon 8 and the catalytically important tyrosines are not defined unequivocally. A different issue associated with the atomic details of the modeled active sites is illustrated by the predicted position of the catalytically involved Asn887. As predicted, the side-chain of this residue is pointing by $90^{\circ}$ away from the peroxide-heme axis and thus would render this a residue incompetent for catalytic turn-over. Nevertheless, analysis of the respective valine-variant indicated that this residue is crucially involved in the hydroperoxide rearrangement reaction. Although the position of this residue might be refined by a readjustment of the entire I-helix, an eventually more likely explanation for a repositioned amide side chain is given by a kink interrupting the helical secondary structure of the I-helix above the active site in virtually all cytochrome P450s (Denisov et al., 2005). In the thus created space the catalytic threonine of typical P450s is located. A similar kink and the resulting readjustment of catalytically important amino acids was also reported for hydroperoxy fatty acid rearranging P450s (D.-S. Lee et al., 2008). Nonetheless, the predicted structure of PpoA's P450-domain contains an unperturbed I-helix and thus might be false predicted in this detail. As a last example for the putatively wrong predicted details of the active-site structures, the position of the hydroperoxy fatty acid substrate in the P450-domain is mentioned here. Although two ortholog classIII-P450s have been crystallized with the same substrate-analogue, the resulting structures reveal two completely different substrate orientations (L. Li et al., 2008; D.-S. Lee et al., 2008) making the evaluation of the native binding modes nearly impossible and preventing a reliable prediction of substrate position in the P450-domain of PpoA. 
Despite these problems associated with various details of the predicted active-site structures, the thorough biochemical and biophysical characterization of variants of certain hypothesized determinants proved the functional role of the respective amino acid position (Section 4.4). Utilizing this approach five amino acids crucially involved in substrate conversion by PpoA were identified. Of these five residues two are apparently involved in dioxygenation, while the remaining three amino acids govern hydroperoxy fatty acid rearrangement in the P450-domain of PpoA. It should be pointed out that all identified amino acid determinants are highly conserved throughout the whole Ppo-enzyme family and thus might serve as prototype determinants that characterize the reactivity of the whole enzyme family. The involvement of the mentioned determinants in catalysis is discussed in detail within the following two subsections. Elaborating on the active site structures and to get further insight into the macromolecular assembly of the predicted domains to an entire oligomeric enzyme, small-angle $\mathrm{X}$-ray scattering data were used to derive a low resolution structure of the native enzyme (Section 4.6.1). The thus postulated quaternary structure of PpoA is discussed in Section 5.1.3.

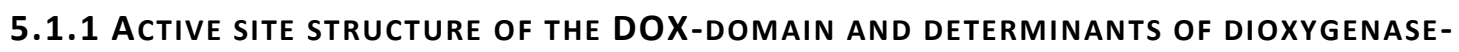 ACTIVITY}

The N-terminal domain (amino acid 1-620) of PpoA was assigned by sequence homology as member of the myeloperoxidase enzyme family (Daiyasu and Toh, 2000) and by multiple sequence alignments this study could identify an eighteen amino acid long sequence motif (Section 4.3.1.1) within the DOX-domain that is highly specific for all PpoA-homologue enzymes and thus might guide a way for enzyme classification within the myeloperoxidase family. Based on structures of different myeloperoxidases, the $\mathrm{N}$-terminal domain of PpoA was modeled and found to resemble the crystal structures of the characterized heme-dioxygenases PGHS-1 and PGHS-2 (Section 4.3.1.1; Figure 15). The cofactor and the fatty acid substrate (linoleic acid) were placed in the same position as it is found in PGHS, which leads to a spatial arrangement, in which all known determinants of reactivity are placed in reasonable geometry. The heme is coordinated by a proximal histidine (His377) and on the distal side a second histidine (His202) can be found. The corresponding positions of both histidines were already shown to be crucially involved in enzyme activity of the homologue 7,8-LDS (Garscha and Ernst H Oliw, 2008) and PpoA's His377Ala-variant is also inactive (Dr. Florian Brodhun, personal communication). The fatty acid is bound within a hydrophobic channel (Figure $17 \mathrm{~A}$ ) of which Val328 next to the fatty acids $\mathrm{C}_{4}$ has been implemented to be involved in the regioselectivity of hydroperoxide formation (Garscha and Ernst H Oliw, 2009; Brodhun et al., 2010). Besides hydrophobic interactions of the fatty acid tail with apolar amino acids lining the substrate channel, the carboxylate of the substrate might be bound by ionic interactions to the side chain of a basic amino acid. A similar binding mechanism is proposed for both homologue heme dioxygenase: $\alpha$-DOX and PGHS (Koszelak-Rosenblum et al., 2008). Although at the position homologue to PGHS-2's Arg120 no basic amino acid was identified within the predicted PpoA-structure, the side chain of Arg336 is 
located at the entrance of the proposed substrate channel and resides at the same distance to the substrate's carboxylate as Arg120 in mPGHS2 (Figures $15 \mathrm{C}$ and $17 \mathrm{~A}$ ). Therefore, it was assumed that this residue might confer affinity of the fatty acid substrate to PpoA's DOXdomain. To validate this hypothesis, a respective variant with an uncharged amino acid at position 336 (Arg336Met) was constructed and its substrate affinity was measured by a kinetic approach and compared to wild type enzyme (Section 4.4.1, Figure 18). Although the measured kinetics of the variant are altered in a way supporting the hypothesis of Arg336 being involved in substrate binding, the effects of this mutation and especially the increase of $k_{m}$ are not as pronounced as one would expect for a residue playing a pivotal role in substrate binding. On the one hand, the small magnitude of the observed effect might be either caused by the enzyme's complex kinetics, which is governed by activation- and inactivation-processes that prevent reaching steady state conditions and hence complicate the evaluation of the kinetics by Michaelis-Menten theory (discussed in more detail in section 5.2), or by the fatty acid substrate forming micelles leading to an underestimation of the true $\mathrm{k}_{\mathrm{m}}$. Besides these putative systematic errors, one should also keep in mind that the shape of the dioxygen depletion kinetics measured for the Arg336Met-variant indicates a transition towards a different oxidation-mechanism caused by this mutation. Although a sigmoidal description of the measured data leads to a significantly improved fit, a clear conclusion why the conducted mutation should affect the enzyme's cooperativity in such a drastic way is not easily derived. Interestingly, sigmoidal description of the measured variant-kinetics does not lead to changed values of $k_{m}$ and $v_{\max }$, relative to the wild type values, but affects merely the Hill coefficient. While this effect might be explainable for the Arg336Met-variant, a variant proposed to have affected binding properties; the observation that the Tyr327Phe-variant possesses a similar effect (Figure 19) renders this hypothesis implausible again and suggests that a lower activity may just unmask an intrinsic cooperative enzyme behavior. Albeit this possibly changed enzyme cooperativity, the $k_{m}$ and $v_{\max }$ of the Arg336Met-variant, as compared to the wild type, are also not altered more significantly for the sigmoidal described kinetics. Thus the small observed effect could have a mechanistic implication and point out that the basic amino acid in the DOX-domain of PpoA rather resembles the function of Arg120 in PGHS-2 than that of the homologue position in PGHS-1. In isoform 2 of this heme DOX the positively charged side chain of Arg120 does not ionically interact with the substrate, but stabilizes its position by formation of weaker hydrogen bonds and thus renders hydrophobic interactions with the fatty acid tail relatively more important (Rieke et al., 1999). In line with other determinants crucially involved in positioning of the fatty acid are oxidations of unusual substrates (e.g. 14:1 $1^{\Delta 9 z}, 16: 1^{\Delta 9 z}, 18: 1^{\Delta 8 z}, 18: 1^{\Delta 11 z}, 18: 1^{\Delta 12 z}$ and 20:1 $1^{\Delta 11 z}$ ), which indicated that the regiospecificity of the oxidation might be determined rather by the distance from the double bond (system) than by the distance from the substrate's carboxyl- or $\omega$-end (Brodhun et al., 2009; Ernst $\mathrm{H}$ Oliw et al., 2011). This finding implicates that the regioselectivity of the dioxygenation within the DOX-domain might depend on the correct placement of the substrate's double bonds. Based on this observation, Tyr327 can be identified as solely aromatic residue putatively determining the placement of the substrate by $\pi$-stacking to assure that the 
dioxygenation occurs regio-selective. Remarkably, this residue is highly conserved throughout all dioxygenases and was suggested to be involved in a hydrogen bonding network that places the catalytic competent tyrosyl radical in a position that resembles a rotamer perfectly placed to abstract hydrogen from the fatty acid substrate (Rogge et al., 2006). In contrast to this clear attribution of a certain role, Thuresson et al. speculated that the corresponding COX-residue might rather be involved in placement of the substrate than positioning of the oxidizing tyrosyl (Thuresson et al., 2001). This deduction was based on and is strengthened by the observation that in COX and 7,8-LDS only variants in which the Tyr was replaced by Phe, as another aromatic amino acid, remained active (Thuresson et al., 2001; Garscha and Ernst H Oliw, 2008). Nevertheless, since a narrower ß-proton splitting of the tyrosyl radical was observed in the EPRspectrum of the Tyr327Phe-variant, which indicates a conformational perturbation of the oxidizing tyrosine, it is reasonable to conclude that Tyr327 in PpoA contributes, at least partially, to proper positioning of the oxidizing tyrosyl radical. Hence, a role of this residue in substrate positioning would be not more than an additional one and evaluation of the mechanistic implications of this residue is thus complicated. Moreover, preliminary results characterizing PpoA's Tyr327Leu-variant, which should not contribute to substrate binding by $\pi$-stacking, indicated that the regioselectivity of dioxygenation by this variant is not less specific and thus Tyr327 is most likely not crucially involved in substrate-placement. Besides the already mentioned function of Tyr327 to place the oxidizing tyrosine radical in a catalytic competent conformation, the spatial arrangement of Tyr327 next to the substrate's $C_{8}$ furthermore suggested that the catalytic tyrosyl radical might be (alternatively) formed at this position and the previously implicated Tyr374 (Brodhun et al., 2009; Fielding et al., 2011) might be only a transient link in a radical chain for intra-molecular electron transfer from Tyr327 to heme compound I. To test this hypothesis, radical distances between the tyrosyl-radicals in the distinct domains were measured by DEER (Section 4.6.2) and compared to the tyrosine distances derived from PpoA's low resolution quaternary structure (Section 4.6.1). Although the distance distribution obtained for the wild type enzyme indicated the presence of an additional minor distance that could be interpreted as a second or alternative radical site at Tyr327, the distances extracted from the dipolar evolutions measured for the Tyr327Phe-variant were basically identical to the wild type pattern and thus a second radical at this position is rather unlikely (Figure 33). Integrating the results from the DEER-experiment of the Tyr327Phe-variant and the modeled active site structure with solely two tyrosines being in reasonable distance to eventually oxidize the substrate, the assignment of Tyr374 as catalytic competent residue is further strengthened. 


\subsubsection{ACtive Site Structure Of the P450-domain and Determinants OF Hydroperoxy FATTY ACID ISOMERASE-ACTIVITY}

Based on the observation that a basic amino acid is, at least partially, involved in substrate binding to PpoA's DOX-domain, the contributions of charge-charge interactions to binding in the P450-domain were investigated. Strikingly, no positively charged amino acid, which could interact with the hydroperoxy fatty acid's carboxyl group, was identified in the proposed active site of the P450-domain and the proposed binding mechanism with the carboxyl group in the active site suggests that the substrate has to be protonated (Figure $16 \mathrm{~B}$ and $17 \mathrm{~B}$ ). Albeit this might be indicative of a false predicted structure and binding mechanism, the possibility that this is a valid observation was further investigated by conversion of an uncharged substrate analogue (Section 4.4.3). The methyl ester of 8-HPODE was not only converted without altered reaction specificity, which points out that a hypothetical ionic interaction is not crucial for substrate placement and hence for regioselectivity of the rearrangement reaction, but also a competitive conversion of 8-HPODE methyl ester and free 8-HPODE revealed that there is no quantitative difference in substrate binding of these both substrates (Figure 20). Contrary, conversion of 8HPODE at $\mathrm{pH} 9$ indicated that the carboxylate actually has to be protonated for substrate rearrangement. This might be explained by repulsion of the deprotonated, anionic substrate from the uncharged active site of the P450-domain. Considering that ionic interactions do not contribute to substrate-binding to the P450-domain, a new aim was to explore the contributions of distinct binding modes to substrate affinity. Especially taking into account that fatty acid hydroxylations by PpoA will occur specifically on carbons -1 and -4 relative to the substrate's double bond (Brodhun et al., 2009), one might speculate that $\pi$-stacking between an aromatic residue and the substrate's double bond(s) contributes to proper positioning of the substrate within the active site. Within the originally modeled "opened" P450-structure indeed two phenylalanines (Phe795 and Phe799) are in reasonable proximity to 8-HPODE's $\Delta 9$ and $\Delta 12$ double bonds for $\pi$-stacking to occur (Section 4.3.1.2; Figure 21). The substrate was placed in this position based on the substrate's electron density found in guayule AOS (L. Li et al., 2008). However, the distance between the peroxide and the heme renders this position most likely catalytic inactive. Therefore, assuming that the two phenylalanines indeed are involved in substrate binding, an assumption that was later on experimentally verified, a conformational change involving a movement of the enzyme's F/G-helix towards the heme and thus closing the active site upon substrate binding would be mandatory. Actually, similar structural perturbations have been shown to occur upon substrate binding to P450-BM3 $(\mathrm{H}$. Li and Thomas L. Poulos, 1997, 1999) and several other cytochrome P450s (Pochapsky et al., 2010). With two reasonable amino acid determinants for substrate binding on hand, the next step was to biochemically characterize the respective enzyme-variants (Section 4.4.3). Because no assay to continuously monitor either depletion of the fatty acid peroxide or formation of the dihydroxy fatty acid is

feasible, the kinetics of the P450-domain cannot be measured directly. Instead, end-point measurements of the enzymatic fatty acid conversion by PpoA were conducted and a decreased 
activity was deduced from an accumulation of hydroperoxy fatty acid, while an impaired and less constrained substrate binding might be visualized as a loss of reaction specificity and a diversified product pattern. Although the Phe795Leu-variant showed the expected biochemical characteristics with a less specific regioselectivity of the rearrangement reaction, which was more pronounced for conversion of oleic acid, a substrate with solely one constraining double bond, the Phe799Leu-variant showed a surprising effect on the resting enzyme (Figures 22, 23 and 24). All available data are consistent with a severely decreased heme occupancy of the P450-domain of this variant. Concomitant the product pattern of the rearranged hydroperoxy fatty acid was even more diversified as compared to the Phe795Leu-variant. Interestingly, only the variant with a leucine at position 799 exhibited the reduced heme occupancy, while all other amino acids tested at this position (i.e. Met, Trp and Tyr) did not perturb the cofactor binding. Despite the unperturbed heme-binding, the additional variant with an aliphatic amino acid (Phe799Met) rearranged 8-HPODE also to a diversified product pattern (Figure 25). This observation indicates a dual function of Phe799 in substrate as well as cofactor binding. Nevertheless, it has to be admitted that an explanation of how an amino acid at the distal side of the heme could affect cofactor binding in such a drastic manner is not straightforwardly given. Likely the change of phenylalanine to leucine provokes a severe perturbation affecting the structure of the whole domain. Although the influence of the respective variants, having an aliphatic residue at this position, on the rearrangement's regioselectivity seems to be more obvious: lacking of the proposed $\pi$-stacking leads to a less constrained substrate position and thus a higher probability of other parts of the substrate to be oxidized, the fact that Phe799 is more important for regioselectivity of the rearrangement than Phe795 is not consistent with this hypothesis. From their arrangement within the structure, it would be expected that the $\Delta 9$ double bond of the substrate is bound by the benzyl ring of Phe795 and regiospecific rearrangement of the 8-hydroperoxy derivative of oleic acid by wild type enzyme suggests that proper placement of this double bond is more important relative to the placement of the $\Delta 12$ double bond of polyenoic substrates. Additionally, the Phe799Leu-variant rearranged 8-HPOME also to a diversified product pattern and not specifically to 5,8-DiHOME, as would be expected if the aromatic ring at position 799 would be solely necessary to place the substrate's $\Delta 12$ double bond. Therefore, Phe799 might possess a distinct function that is not $\pi$-stacking to the $\Delta 12$ double bond. Located at the boundary of the active site, its bulkiness might constrain the substrate in a catalytic competent position (Pochapsky et al., 2010). In line with this observation, the variant with a bulkier residue at position 799 (Phe799Trp) did not rearrange significant amounts of hydroperoxy fatty acids, putatively caused by a restricted access to the active site. Alternatively this phenylalanine might mediate substrate access and product egress. A similar function of a phenylalanine was proposed for a variety of cytochrome P450s among them Cyp3A4, Сyp2B1 and P450cam (Fishelovitch et al., 2009). Nevertheless, for these enzymes the gate controlling the access to the active site is build up by a pair of phenylalanines that is closed by $\pi$-stacking between the distinct benzyl rings. In the predicted structure of PpoA's P450domain Phe1059 might serve as complementing residue forming this gate. However, the 
Phe1059Leu-variant did not show a diversified product pattern, but its reaction specificity was comparable to wild type enzyme (data not shown).

Despite the identification of Phe795 and Phe799 as residues involved in determination of the rearrangement's regioselectivity, identification of determinants responsible for differentiation between 5,8-LDS and 7,8-LDS was not successful. In order to identify potential sites determining the reaction specificity of a cytochrome P450 enzyme, one can start to investigate the so called substrate recognition sites (SRSs). These SRSs have been defined as flexible parts of the enzyme governing the accommodation of different substrate molecules and specifying their regio- and stereo selective metabolization (Gotoh, 1992). While most of these SRSs seem to be responsible for adaption towards a highly diverse range of substrate sizes, SRS 5 is one of the most important sites for distinct regio- and stereoselectivity in closely related enzymes (Seifert and Pleiss, 2009). Therefore, its potential involvement in the differentiation between 5,8-LDS and 7,8-LDS was investigated. SRS 5 starts C-terminal of the K-helix directly behind the ExxR-motif and spans over 9 to 11 amino acids to an arginine, which stabilizes the heme by interaction with one of the propionate groups (Seifert and Pleiss, 2009). Within the modeled structure of PpoA's P450-domain, this region is indeed adjacent to the relevant part of the substrate and might be involved in determination of regiospecificity. Nevertheless, the three residues in direct contact with the substrate (Val940, Ala941 and Leu942) are conserved between 5,8-LDS and 7,8-LDS. Thus, it seems unlikely that SRS 5 is involved in the differentiation between these two enzymes. Instead of single amino acid determinants, significant changes in the architecture of the protein fold might be also responsible for an altered regioselectivity. A sequence alignment shows that within the cytochrome P450-domain of 7,8-LDS three longer inserts exist as compared to 5,8LDS. These inserts in the P450-domain are solely responsible for the longer amino acid sequence of 7,8-LDS in comparison to 5,8-LDS (1165 amino acids vs. 1081 amino acids). To assess a putative effect of these inserts on the regioselectivity of the catalyzed rearrangement reaction, the cytochrome P450 domains of both enzymes have been modeled with the I-Tasser algorithm. However, within these putative structures none of the three additional loops of 7,8-LDS interfered with the predicted substrate binding site of $\mathrm{PpoA}$ and thus a conclusion whether one of these loops might be responsible for the different regioselectivity in the distinct enzymes is not possible. Based on the predicted substrate binding mode, an alternative hypothesis to explain the differences between 5,8-LDS and 7,8-LDS was that in 5,8-LDS a six-membered transition state enabling the proximity of $C_{5}$ and $C_{8}$ is formed, while the substrate is not forced to adopt this conformation in 7,8-LDS (Section 4.5, Figure 28). Although Met791 was identified as putative structure fulfilling this function in $\mathrm{PpoA}$, a respective variant did not catalyze formation of 7,8-DiHODE. Besides a structural explanation, one might consider that differences in the cofactor chemistry could also explain the altered reaction specificity. Exemplary a short lived compound I might readily oxidize the closest carbon, while a less reactive compound I might be stable enough to oxidize the substrate not before it was repositioned. However, with exception of the thiol ligand, the factors governing the reactivity of $\mathrm{P} 450$-heme are not well understood yet 
and one of the residues in the heme-binding loop that was proposed to mediate this reactivity did not influence the regioselectivity of fatty acid dihydroxylations catalyzed by PpoA (Section 4.5).

Upon binding of the substrate, the peroxide has to be reduced and concomitantly the P450 heme is oxidized to yield compound I, the reactive species capable to hydroxylate an unactivated $\mathrm{C}-\mathrm{H}$ bond (Rittle and Green, 2010). In related hydroperoxy fatty acid rearranging cytochrome $\mathrm{P} 450 \mathrm{~s}$, this peroxide cleavage was proposed to be facilitated by a hydrogen bonding network involving the amide function of an asparagine located in the enzyme's I-helix on the distal side of the heme (Hecker and Ullrich, 1989; Yeh et al., 2005; D.-S. Lee et al., 2008). While no strictly conserved asparagine exists at the homologue position in PpoA's P450-domain, a neighboring residue in the I-helix of this enzyme-domain was found to be an asparagine (Asn887; Section 4.3.1.2; Figures $16 \mathrm{C}$ and $17 \mathrm{~B}$ ). Assuming that this detail of the structure might be false predicted, a respective variant was constructed and characterized biochemically (Asn887Val; Section 4.4.4). As one would expect, if the hydroperoxide rearrangement reaction is impaired, products of the DOX-domain were accumulated in linoleic acid conversions by this variant and concomitantly the amount of produced 5,8-DiHODE was decreased by about $90 \%$ as compared to wild type enzyme (Figure 26). Furthermore, this variant showed a slightly decreased heme content. This weakened cofactor binding is in agreement with the amide side-chain forming a hydrogen bonding network between the peroxide-substrate and the heme-iron and thus representing an additional weak and noncovalent contribution to cofactor as well as substrate binding. Additionally supporting this hypothesis, the g-values that are sensitive to the coordination environment of the iron were found to be slightly altered (Figure 27). To further probe whether the altered position of the asparagine in PpoA as compared to other class III P450s is an artifact of the modeling process or putatively reflects a true structural difference, the binding affinity of a small ligand (imidazole) to the heme was measured. The carboxamide of the catalytic competent asparagine was implemented to shield the distal heme-side and thus cause the merely transient and weak formation of the enzyme-family archetypical absorption of the reduced CO-complex at $450 \mathrm{~nm}$, which is typical for class III P450s (Yeh et al., 2005; D.-S. Lee et al., 2008). By this mechanism also binding of imidazole is less pronounced than in typical P450s. Although a transient formation of the reduced CO-complex absorbing at $450 \mathrm{~nm}$ was already shown in a previous study (Brodhun et al., 2009), these results are not sufficient to quantitatively assess this process. Therefore a spectrally monitored imidazole-binding study was conducted. The evaluation of the obtained data was complicated by the existence of a second heme. Although this complexity might be theoretically overcome by considering a two-site binding model, the distinct spectral parameters of the histidine and cysteine coordinated heme, with the soret peak of the DOX-domain slightly blue-shifted in comparison to the P450 heme, render also this approach impracticable, as the read out of the assay is not independent from the binding site. Despite these complications, the tendencies revealed by the imidazole titrations indicate that i) imidazole has a higher affinity to the P450-heme of PpoA as compared to the 
active site of prostacyclin synthase (Yeh et al., 2005) and ii) the mutation of Asn887 to valine did not significantly increase the binding affinity of imidazole. Both results might be interpreted as indications that the asparagine in PpoA shields the heme less strictly as in other class III P450s and thus mutating this residue to a less bulky one does not significantly ease the access to the heme. This conclusion implements that the modeled structure might predict a true position of this asparagine and that the active site of PpoA's P450-domain is more open than in other class III P450s and thus could enable the access of water. The presence of these water molecules in turn could be the reason why the hydroperoxide cleavage occurs heterolytically in fatty acid diol synthases (Kupfer et al., 2001) and not homolytically as in other class III P450s (Brash, 2009). At least, acid catalyzed cleavage by water was shown to be the main determinant of hetero- vs. homolytical O-O bond scission in P450 2B1, 3A1 and 3A5 (Correia et al., 1995). Although the present study probed only the involvement of an I-helical asparagine's amide in peroxide cleavage, another mechanism for peroxide cleavage, which can be identified in CYP152B1 and CYP152A1 (Matsunaga et al., 2002; D.-S. Lee et al., 2003; Fujishiro et al., 2011) and which requires an additional carboxylic function, seems to be unlikely, since no carboxylate was identified in the proximity of the peroxide, to be cleaved during catalysis.

\subsubsection{QUATERNARY STRUCTURE}

PpoA and related enzymes were thought to be tetrameric for more than ten years (Chao Su and Ernst H. Oliw, 1996), but the SAXS-data obtained in this study illustrate that this view on the molecular weight was not detailed enough. Both, the zero-angle scattering intensity $I(0)$ as well as the $a b$ initio model of the particle's shape calculated from the entire scattering curve, suggest that native PpoA forms rather a trimer (Section 4.6.1; Figure 29). The flat, trimeric quaternary structure causes an overestimation of the native molecular weight determined by size exclusion chromatography, which is calibrated for globular proteins. The derived quaternary structure, which's envelope is based on the experimental obtained SAXS data, can reasonably explain at least two observations that have been made for PpoA: i) Only the edges of the flat molecule can reach into the active site of trypsin. Thus, the digestion of native PpoA does not lead to a complete degradation, but yields stable fragments (Section 4.6.1; Figure 30). ii) The arrangement of the monomers within an equilateral triangle (Figure 31) explains why determination of the distances between tyrosyl-radicals of the monomers gives only one significant signal representing a distance of $\sim 5.2 \mathrm{~nm}$ ((Fielding et al., 2011) and Section 4.6.2; Figure 33). The distance observed with this technique in frozen solution agrees quite well with the distance of the tyrosines, putatively forming the radical, in the model. The distance differences between both methods might be explained by a genuine difference between the liquid and the frozen solution. Still one should keep in mind that for SAXS a difference as small as $10 \AA$ is not resolvable. An additional modulation of the dipolar evolution representing a second, smaller distance between paramagnetic species of $\sim 4.1 \mathrm{~nm}$ indicated that an alternative radical site might be formed. However, the same distance distribution of the Tyr327Phe-variant as 
compared to the wild type indicated that at least this tyrosine is not responsible for the additional distance observed. At the same time, this opens the question where this smaller distance might arise from. Theoretically one could speculate that the tyrosyls might exhibit a certain conformational flexibility. However, to span a distance difference of $10 \AA$, two tyrosines would have to undergo a severe and concerted movement towards each other and an intermediate or broadened distance would be expected in this case. Moreover, the catalytic active tyrosine of the homologue PGHS was shown to posses no conformational flexibility (Sidhu et al., 2010). Considering that this smaller distance was observable even after the fast quenching ( 4 to 6 seconds) performed on the here investigated samples, it seems to be unlikely that it is a distance associated with radical side products not involved in catalytic turn-over or responsible for enzyme-inactivation. Additionally strengthening the hypothesis that this minor radicaldistance is not caused by dipolar coupling to another protein radical is the observation that the tyrosyl radical formed upon activation of the wild type enzyme can be simulated by a single tyrosine (Fielding et al., 2011). To finally rule out the possibility of an additional radical site indicative of enzyme inactivation, a rapid freeze-quench sample could be characterized in the future. Although also dipolar coupling to the low spin heme rather than to a distinct radical could theoretically explain the existence of a second distance (Figure $34 \mathrm{~A}$ ), the fact that this smaller distance is not observed when the pump-pulse is applied in closer resonance with the low-spin heme indicates that this is no valid hypothesis. One should also keep in mind that, despite that the resting enzyme should posses a symmetric structure, substrate binding in the course of the activation process might induce a conformational change, which could perturb the structure and eventually lead to an isosceles triangle rather than an equilateral one. To probe this hypothesis a future experiment could determine tyrosyl-radical distances in a sample activated with hydrogen peroxide. Since this peroxide is no substrate for the P450-domain of the enzyme, no postulated conformational change should occur and thus also the activated enzyme should posses a symmetric geometry. An alternative explanation for the existence of the smaller distance might be that it is merely an artifact caused by the treatment of a triple-spin system as biradical. It was shown that this simplification can lead to a broadening of the distancedistribution and the extraction of small distance artifacts especially from tri-radicals with an equilateral geometry (Gunnar Jeschke et al., 2009). Nevertheless, also treatment of the data with a model describing an equilateral tri-radical does not meet the requirements of the investigated system. This stems from the fact that the spins in the investigated system are only transiently formed and not stable. Hence, it is not clear to which extent the mean value of $\sim 0.6$ spins per trimer is distributed as mono-, bi and tri-radical. Although a cooperative enzyme behavior favoring the formation of bi- and tri-radicals is possible, most likely a mixture of different radical populations might exist complicating the extraction of true distance distributions further. Moreover, the existence of additional paramagnetic centers in the nanoobject (i.e. high-spin and low-spin heme) might imply the need to consider further spin correlations. Interestingly, the smaller distance exhibits quite exactly the double frequency as compared to the larger distance $\left(v_{\text {dd }}(4.12 n m) / v_{\text {dd }}(5.18 \mathrm{~nm})=1.99\right)$ and thus might be indeed an 
artificial sum combination of this true distance. Taking into account that distortions of the extracted distances are most pronounced for symmetric/equilateral spatial arrangements of spins (Gunnar Jeschke et al., 2009) and that the absence of the low-spin heme might not only lead to a reduction of the complexity of the multi-spin correlations, but also to a structural perturbation eventually destroying the molecule's symmetry, also this hypothesis might be in agreement with the result of PpoA's Phe799Leu-variant. Generally, the absence of the smaller distance in this variant (Figure 34) did not substantiate or falsify one of the several hypotheses explaining the nature of this distance. The loss of the P450-heme could prevent formation of an additional protein-radical in this domain and the associated diminished activity of the rearrangement reaction could suppress the manifestation of conformational changes, occurring upon substrate binding, as perturbed symmetry of the oligomer. Everything considered the nature of this additional distance cannot be clarified at the present stage of research.

Altogether, the SAXS-derived low-resolution structure, validated by tryptic digest and DEERconstraints, helped to set the proposed structure of PpoA to a new level. Although an atomic view of the interactions between the monomers could not be given, the orientations of both domains with respect to each other were derived from the predicted domains docked into the SAXS-derived low resolution structure and the assembly of two conserved, oxylipin forming activities in a single polypeptide chain as well as its quaternary structure were characterized for the first time.

\subsection{Kinetic isotope effects to probe the kinetic contribution of hydrogen-transfer steps}

Utilizing regioselectively deuterated oleic acid, the kinetic contribution of the distinct hydrogen transfer steps within PpoA's reaction mechanism were probed. The proposed reaction mechanism (Brodhun et al., 2009; Fielding et al., 2011) predicts at least three different steps of hydrogen transfer: i) the catalytic competent tyrosyl radical abstracts hydrogen from the substrate's $\mathrm{C}_{8}$; ii) this hydrogen is subsequently retransferred to the intermediately formed peroxyl radical thus restoring the tyrosyl radical for the next catalytic cycle and iii) within the P450-domain an activated heme species, most likely compound I, has to abstract hydrogen from $\mathrm{C}_{5}$ in order to enable oxygen rebound from the oxidized heme. The kinetic contributions of these steps might be revealed by oleic acid dideuterated at $C_{8}$ (to unravel the importance of steps $i$ and ii) and a distinct probe dideuterated at $C_{5}$ (to estimate the influence of step iii on the overall kinetics).

In a first attempt, dioxygenation kinetics were obtained by continuous measurement of dioxygen depletion (Section 4.7.2; Figures 36 and 37). In order to kinetically describe the data obtained for different substrate concentrations, the transient point of maximal velocity was obtained from dioxygen depletion kinetics of each substrate concentration and several models were considered for their evaluation. Obviously, a classical Michaelis-Menten description of the kinetics is not 
valid, since the enzyme system exhibits no steady state. On the other hand, the oligomeric structure of the enzyme suggests cooperative effects and the corresponding sigmoidal behavior of the kinetics would be reasonable as well. Moreover, more sophisticated kinetic models that can be used to describe phase transitions at certain substrate-concentrations (e.g. the formation of micelles and different enzyme kinetics for the free fatty acid and the micelle) might be considered. Also, one should keep in mind that the kinetics might depend on the concentrations of both substrates, fatty acid and dioxygen, as it was described for exemplary for PGHS-1 (Mukherjee et al., 2007). Nevertheless, for a detailed investigation, which of the mechanisms is best suited to describe the fatty acid conversion kinetics of PpoA, highly precise kinetic data are mandatory. Inter alia caused by impaired substrate solubility in aqueous solution these are hard to get and typically the measured kinetics have a rather high standard deviation rendering the credible evaluation of different models exhibiting merely subtle differences impossible. In order to overcome these problems a pragmatic approach was chosen and the kinetic behavior was described by a hyperbolic equation resembling the one known from the classical MichaelisMenten mechanism. Despite the fact that trends arising from differentially deuterated substrates might be revealed by this approach, one should keep in mind that the reaction is dominated by enzyme-activation and -inactivation processes and thus does not exhibit a steadystate. Hence the reaction does not obey a Michaelis-Menten mechanism and the obtained kinetic parameters ( $k$ and $v$ ) have not the classical meaning of $k_{m}$ and $v_{\max }$. For the $C_{8}$-labeled substrate $v_{H} / v_{D}$ was determined to be 33.4 , which is significantly higher than the value of $\sim 7$ that one can expect for bond fission by a semi classical mechanism (Ranaghan and Mulholland, 2010). Nevertheless, it has to be considered that the labeling was only regio-and not stereo selective. Hence, the measured KIE is a combination of primary and secondary KIE. Moreover, the hydrogen/deuterium is proposed to be transferred twice in a full dioxygenation cycle. First, the label is removed from the fatty acid and reacts with the catalytic active tyrosyl radical to form a tyrosine. Following formation of a peroxyl substrate radical by trapping of molecular oxygen by the carbon centered fatty acid radical, the same hydrogen/deuterium is transferred to yield the product of fatty acid dioxygenation and restore the tyrosyl radical for the next catalytic cycle (Brodhun et al., 2009). For both homologue enzymes, PGHS and $\alpha$-DOX, it was shown by measurements in deuterated water that on the time scale of catalysis exchange of the tyrosine's proton with the buffer is negligible and thus indeed both steps of hydrogen/deuterium transfer have to be considered (Mukherjee et al., 2010; Danish et al., 2011). Keeping these contributions to the macroscopically determined KIE for conversion of $\mathrm{C}_{8}-\mathrm{d}_{2}-\mathrm{OA}$ as well as the standard deviations of the measured data in mind the determined value seems not significantly increased enough to claim "hydrogen tunneling" unequivocally. Nevertheless, the observed $\mathrm{V}_{H} / \mathrm{V}_{\mathrm{D}}$ strongly suggests hydrogen tunneling contributions for breakage of the carbon hydrogen bond. To prove this hypothesis a more detailed investigation of the KIE's temperature dependency is essential. While unequal Arrhenius-prefactors for both isotopologues are typically a sufficient indication for hydrogen tunneling, one should keep in mind that in certain cases this is not enough and additional experimental evidence (e.g. evaluation of multiple KIEs with the Swain-Schaad 
relationship) is needed to unequivocal proof hydrogen tunneling (S.C. Sharma and Klinman, 2008). Nevertheless, a first rough estimation of the temperature dependency of the reaction of PpoA with oleic acid and $\mathrm{C}_{8}-\mathrm{d}_{2}-\mathrm{OA}$ indicated that the Arrhenius-prefactors for conversion of both substrates are equal and thus contradict the possibility of hydrogen tunneling being involved in the abstraction of hydrogen from the substrate's $C_{8}$ (Section 4.7.4).

Interestingly, the observed pronounced KIE for hydrogen abstraction from $\mathrm{C}_{8}$ and (putative) proceeding of this process by hydrogen tunneling is in agreement with observations for several other fatty acid dioxygenases. LOXs are known for their high deuterium KIEs in the range of $\sim 80$ (Rickert and Klinman, 1999). Notably, although the substrates and products of this enzyme-class are comparable to those of PpoA's DOX-domain, the enzyme mechanism differs. Exemplary the oxidizing species of LOX enzymes is not a tyrosyl radical, but Fe(III)-OH (C. Schneider et al., 2007) and the hydrogen is abstracted from a bis-allylic carbon that exhibits a lower bond dissociation enthalpy than the allylic $\mathrm{C}_{8}$ of the PpoA substrates (Ernst $\mathrm{H}$ Oliw et al., 2010). Interestingly, the two enzymes that are mechanistically comparable to PpoA's DOX-domain are quite diverse with respect to their KIEs. While $\alpha$-DOX was shown to exhibit tunneling effects comparable to LOXenzymes (Gupta et al., 2008a), the situation for PGHS differs remarkably. Although the macroscopically observed KIE was shown to be unexpectedly low more than 40 years ago (Mats Hamberg and Samuelsson, 1967), it was established only recently that this does not reflect a masking of a higher intrinsic value by a complex interplay of certain reaction steps, but merely that the inherent process of bond breaking has a value of just $k_{H} / k_{D} \sim 2$ and thus is significantly lower than expected (Wu et al., 2011). To complete the confusion, the non-native substrate linoleic acid shows a $k_{H} / k_{D} \sim 20$ and thus is within the expected range of deuterium KIEs (Danish et al., 2011). Although one has to consider that the fully deuterated substrate will give a combination of primary and several secondary KIEs and that the hydrogen/deuterium-label has to be abstracted twice, from the fatty acid and subsequently from the catalytic tyrosine, the measured value is in the upper range of what one would expect in a semiclassical mechanism and the authors concluded from several additional experiments that hydrogen tunneling occurs in the course of this reaction. The measured differences between the native (arachidonic acid) and the non native (linoleic acid) substrate might be explained structurally by the geometry of substrate bound to the active site. While the transition state of linoleic acid conversion allows hydrogen to tunnel and permits this pathway for the heavier deuterium, the native substrate is bound in a geometry that allows tunneling of both isotopes and thus reduces the observed KIE. Interestingly, these macroscopically differently expressed deuterium KIEs of the in other respects mechanistically highly comparable $\alpha$-DOX and PGHS suggests that $\alpha$-DOX might be a better model for the PpoA DOX-domain than PGHS. Nevertheless, some other features like the missing peroxidase activity of $\alpha$-DOX (Mats Hamberg, León, et al., 2002) and the suprafacial insertion of molecular oxygen by this enzyme (Mats Hamberg, Sanz, et al., 2002) support the idea that PGHS might be the better suited homologue model and suggest that the DOX-domain of PpoA might mechanistically comprise of aspects from both enzymes. 
In contrast to DOXs, in typical cytochrome P450 enzymes no unique step of catalysis can be identified as conserved rate-limiting step. In fact, depending on the enzyme, several microscopic reaction steps might be rate-limiting (Guengerich, 2002). Although often the second electron transfer step preceding the formation of compound 0 is found to be rate-limiting (Ortiz de Montellano, 2010), this is obviously not of interest for hydroperoxide rearranging P450s. According to Guengerich et al., the rearrangement reaction catalyzed by the PpoA-P450 domain might be rate limited by: $\mathrm{C}-\mathrm{H}$ bond breaking or product release (Guengerich, 2002). The later one is only of importance if the substrate induces a conformational change upon binding, a situation that actually might occur in PpoA. To assess a putative rate-limitation caused by $\mathrm{C}-\mathrm{H}$ bond breaking, $\mathrm{C}_{5}-\mathrm{d}_{2}-\mathrm{OA}$ was utilized to probe the kinetic contribution of hydrogen abstraction on the overall reaction. Surprisingly dideuteration at this carbon did not only influence the amount of 5,8-DiHOME (Figure 38) and led to a diversification of rearranged products (Figure 39), but also had an effect on the dioxygenation rate (Figure 36 and 37). Although this indicates that the hydrogen abstraction from $C_{5}$ is the rate-limiting step during PpoA's rearrangement reaction, the influence of dideuteration at $C_{5}$ on the kinetics of dioxygenation, determined as dioxygen depletion, was puzzling and is not explainable by the assumption of domains, acting independent from each other. To understand how an isotope effect, decreasing the reaction rate of a subsequent activity, can influence the reaction rate of a preceding reaction step two explanations are obvious: i) a substrate channel, transferring the substrate from one domain to the other or ii) conformational changes upon substrate binding leading to a perturbation of the structure and allosteric regulation of the activity of the other domain. To rule out the first explanation an isotope dilution experiment was conducted (Spivey and Ovádi, 1999). Assuming a closed channel between both domains, one would expect that labeled linoleic acid would be transferred directly into labeled 5,8-DiHODE and no labeled 8-HPODE would be released to the buffer. At the same time no unlabeled intermediate present in the reaction buffer could enter the P450-domain and thus no unlabeled dihydroxy fatty acid should be detectable. Nevertheless, the results of the corresponding experiment (Section 4.7.5; Figure 40) illustrate that in PpoA no structural closed channel exists for intermediate transfer between both domains. Another mechanism for intermediate transfer between two domains could be the equilibrium of product and substrate between spatially neighbored active sites (Bauler et al., 2010). However, the quaternary structure derived from SAXS-data is not supporting this hypothesis (Section 4.6.1). Although one should keep in mind that the low-resolution of the particle's shape derived from the scattering curve might lead to a misinterpretation of the true domain orientations, these results render a conformational change upon substrate binding to the P450-domain and perturbing the substrate-binding to the DOX-domain the most likely explanation. Additionally, the two structural models obtained for the P450-domain upon prediction of the tertiary structure ("opened" and "closed" form; Section 4.3.1.2) are an indication that these conformational changes indeed might occur upon substrate binding to the hydroperoxide isomerase domain. Nevertheless, these structures are only first indications pointing out that PpoA could show a similar structural perturbation upon substrate binding as P450-BM3. In this 
naturally occurring fusion protein of a cytochrome P450 monooxygenase with its FAD/FMN diflavin reductase substrate binding to the monooxygenase leads to a conformational change affecting the relative orientation of the reductase domain $(\mathrm{H}$. Li and Thomas L. Poulos, 1999). The finding that dideuteration at $C_{5}$ has a more pronounced influence on $V / k$ than on $V$ can be interpreted as a further proof for this hypothesis. Dideuteration at this particular carbon does not influence the measured enzymatic interconversion (i.e. dioxygen insertion yielding 8HPOME), but alters the component describing substrate-binding and formation of enzymesubstrate complex (Northrop, 1975). This might be explained by an altered substrate-binding to the DOX-domain, caused by a changed kinetics of the rearrangement reaction.

\subsection{Electrochemistry of PpoA}

In principle, PpoA immobilized within a DDAB-film on a pyrolytic graphite electrode seemed to be a suitable system to be characterized by protein film voltammetry. In both, cyclic voltammetry as well as square wave voltammetry, oxidation and reduction currents could be measured (Section 4.8, Figure 42). In contrast to the square wave voltammograms the cyclic voltammograms exhibited a more significant reduction peak as compared to the subsequent oxidation peak, which might indicate that the reduction is not fully reversible. The fact that only one peak is observed might indicate that the redox-potentials of the P450- and the DOX-hemes are either not resolvable by protein film voltammetry or that merely one active site was denaturated by the immobilization procedure. Additionally, it should be considered that DDAB might be not a suitable promoter to mediate electron transfer between the electrode surface and the one or the other heme. Albeit neither the DOX-nor the P450-domain remained active upon the immobilization procedure, the value of the measured redox-potential might indicate that rather the DOX-heme was inaccessible for electron transfer by the chosen procedure. This deduction is based on redox-potentials reported for either P450-enzymes (e.g. Cyp121: -476 mV (McLean et al., 2008); CYP125: -303 mV (McLean et al., 2009)) or DOXs (e.g. LOX: $600 \mathrm{mV}$ (Nelson et al., 1995); PGHS1: -167 mV (Goodwin et al., 2000); PGHS2: -156 mV (Goodwin et al., 2000)) compared to the measured midpoint-potential of PpoA (-420 mV).

Although these first results opened in principle the possibility to further characterize the electrochemical behavior of $\mathrm{PpoA}$, including the kinetics of electron transfer, the dependency on temperature and different $\mathrm{pH}$, these investigations were not further pursued. The reason for this is that rather large optimization efforts to identify the best suited electrode material as well as a surfactant that might better mediate the electron transfer are needed. These optimizations are mandatory in order to eventually detect another potential representing the second heme centre. Moreover, some enzymes are reported to exhibit redox parameters quite sensitive to the experimental layout (Goodwin et al., 2000; Johnson et al., 2002). Despite these time-consuming optimization procedures, the main problems arise from the difficulty to physiological interprete the measured potential and obtain mechanistically relevant information (Léger and Bertrand, 2008). An eventually better way to measure adequate redox-potentials would be to use redox- 
titrations as reported in literature (Daff et al., 1997). However, the existence of two heme centers within one protein would make it necessary to monitor spectral changes by EPR-instead of vis-spectroscopy (Verhagen et al., 1995). A final and striking argument against a detailed electrochemical investigation of PpoA is that, also by measuring soluble redox-potentials, one always obtains the electrochemical parameters for the ferric/ferrous-heme transition, a redox transition without physiological relevance for the enzyme mechanism, which is proposed to involve only transitions from ferric to ferryl or oxo-ferryl (Brodhun et al., 2009; Fielding et al., 2011). 


\section{SUMMARY}

Phytopathogenic fungi are not only of economic importance for the yield depression they cause, but can also be considered as severe threat for the health of the consumer ingesting molded crops and fruits. This health threat is caused by several fungal secondary metabolites referred to as mycotoxins (Section 1.2.1). About eight years ago, the group of Nancy Keller proposed that these molding processes, as well as several types of mycoses in mammals, are mediated by fungal hormone-like acting compounds known as Psi-factors (Section 1.2.2). Considering the economic importance of the provoked fungal infections and their impact on public health, a detailed investigation of the underlying mechanisms of Psi-factor perception, their physiological influence and biosynthesis is of scientific significance. Focusing on the biochemical and mechanistic characterization of Psi-factor producing oxygenase $A$ and thus highlighting the molecular basis of Psi-factor biosynthesis, this study could substantiate the mechanism presented by Brodhun et al. (Brodhun et al., 2009) and establish a hypothetical structural model. Although being merely a low resolution model with atomic details based solely on structure prediction and the overall-shape based on small-angle X-ray scattering (Section 4.6.1), several aspects of this structure could be validated by the deployment of adequate biochemical and biophysical approaches. Exemplary the crucial involvement of five newly identified amino acids in determination of the reaction specificity and enzyme activity could be proven (Section 4.4). So it was shown that the fatty acid substrate may bind to the DOX-domain with the $\omega$-end first and is stabilized by ionic interactions of its carboxylate with $\operatorname{Arg} 336$, while binding of the intermediately formed hydroperoxy fatty acid to the cytochrome P450-domain may occur in a reversed orientation, i.e. with the carboxyl-end first, and thus requires an uncharged, protonated substrate. Instead two phenylalanines (Phe795 and Phe799) seem to play a role in substrate-binding and determination of reaction specificity. While the rearrangement reaction catalyzed by the P450-domain involves heterolytic cleavage of a peroxide, which is facilitated by the presence of Asn887, the dioxygenation in the $\mathrm{N}$-terminal domain of PpoA is mediated by a tyrosyl radical. Based on the active site structure, two tyrosines were postulated to be involved in this process. While EPR-spectroscopy of the respective variants revealed that Tyr374 ((Fielding et al., 2011) and section 4.6.2) is most likely the catalytic active residue, Tyr327 seems to be involved in orienting this residue in a catalytic competent position. Besides these active site structures guiding the enzymatic turn-over, a distinct aspect of the enzyme mechanism was evaluated by the use of specifically dideuterated substrates (Section 4.7). Thus it was shown that hydrogen abstraction preceding the insertion of molecular oxygen is the rate limiting step of the overall reaction and both domains of the bifunctional enzyme are not acting independent from each other, but are kinetically linked. Taken together, this study could elucidate the functional and structural basis of Psi-factor biosynthesis in ascomycetes and highlight the molecular background for the physiological role of Psi-factors in host-pathogen interactions, which was established by previous studies (Section 1.2.2). 
Summary 


\section{OUTLOOK AND FUTURE PERSPECTIVES}

To further establish PpoA's reaction mechanism from a mechanistic point of view, detailed investigation of several aspects presented in this work could be conducted. Exemplary, several determinants of substrate-binding were proposed in this study (Arg336; Phe795 and Phe799), but their involvement in substrate-binding was not proven unequivocally. Expanding on this issue a comprehensive study utilizing isothermal titration calorimetry and the various variants could be conducted. Nevertheless, this might be a rather challenging study since chemical similar compounds (e.g. linoleic acid and hydroperoxy linoleic acid) might show affinity to distinct active sites of the enzyme, namely the peroxidase-, the DOX- and the P450-active site. Deciphering the binding-contributions of a given substrate to the distinct sites might be even further complicated by the low solubility of the substrates in aqueous buffer and the concomitant mixing artifacts arising from substrates, solubilized in ethanol. A different valuable investigation of PpoA's reaction-mechanism might involve stopped-flow measurements to kinetically asses the heme reaction cycle in both domains. However, one has to consider that both hemes will form structurally highly similar intermediates and an assignment of spectral features to one or the other domain is not straightforward. Despite this difficulty, some minor differences of the UV/vis-spectra might arise from the distinct heme-ligation. Exemplary, it was shown that compound I of PGHS-1 exhibits an absorption maximum at $630 \mathrm{~nm}$ (Rouzer and Lawrence J. Marnett, 2003), while this maximum is red-shifted by $60 \mathrm{~nm}$ in Cyp119 (Rittle and Green, 2010). A way to more easily overcome the issue of similar heme-intermediates in both domains would be to genetically truncate the enzyme and obtain the distinct activities expressed independent from each other. Besides the problem of achieving this (several attempts have been made during this thesis without success, data not shown), a more severe counter-argument against this strategy is that the kinetics measured for the single domains will not reflect the complex interplay of both domains in one polypeptide chain and thus will fail to address the most intriguing feature of PpoA and related enzymes. Finally, from a biochemical point of view, a detailed investigation of cooperativity effects occurring in the trimeric enzyme might justify some efforts. Starting with theoretical indications that substrate-binding to PpoA's P450-domain might induce a conformational change and supported by the experimentally observed influence of substrate-dideuteration at $C_{5}$ on the dioxygenation rate, first data suggesting an allosteric regulation occurring in the bifunctional enzyme have been collected. To further decipher this putative process, one could try to get better kinetic data with lower standard deviation that might resolve a sigmoidal behaviour of the kinetics. In this respect one should think of overcoming the issues of substrate-solubility by the use of detergents. Additionally, CD-and fluorescence-spectroscopy might be utilized to monitor conformational changes upon substratebinding to the one or the other domain. 
Although in this study a model could be derived that gave first insights into the quaternary structure and template-based structure prediction seems to be a reliable approach to predict atomic details of the distinct domains, a detailed view on the structurally most intriguing part of Ppo enzymes, the linkage between both domains, could not be given. Because several unsuccessful attempts were made to crystallize PpoA, this enzyme appears not to be a suitable candidate to approach the answer to this structural issue. Although some more efforts can be undertaken to finally get a PpoA crystal, e.g. construction of PpoA-variants that might exhibit a reduced surface entropy, a much more promising way to obtain a Ppo crystal seems to be to initiate crystallization trials with homologue enzymes. The high conservation of Ppo enzymes in ascomycetes (Andreou et al., 2009) as well as the existence of PpoA orthologs in virtually all ascomycetes (Tsitsigiannis, Kowieski, et al., 2005), provides a huge diversity to seek for suitable candidate enzymes. Nevertheless, the appropriate enzyme for this experiment might not be predictable rational, but rather involve time-consuming trial and error. Since this involves cloning, expression, purification, characterization and crystallization for each tested candidate, much effort has to be undertaken, if one decides to go this way.

Albeit there are still details of the reaction mechanism to be answered, a much more important field of research seems to be a more detailed physiological characterization of PpoA. While the endogenous role of Psi-factors in ascomycetes to balance sexual and asexual spore development is quite well established (S P Champe et al., 1987; S P Champe and el-Zayat, 1989; Tsitsigiannis, Kowieski, et al., 2004, 2005; Tsitsigiannis, Zarnowski, et al., 2004), its more exciting role in interspecies communication is more elusive. Although initial data were accumulated, which suggest that Psi-factors produced by PpoA are involved in host-pathogen interactions (see section 1.2), no definite proof of this hypothesis was given yet. In the light of the here presented data describing the enzyme's mechanism and structure, a study closing the gap existing between the characterized enzyme and its scarcely defined physiological role might be the scientifically most promising one. The experimental design of such a study could potentially involve heterologous expression of ppo genes in a putative host and characterization of the susceptibility of the resulting transgenic host towards a variety of fungal pathogens in comparison to the non-transgenic host. A correlation of the assumed change in the resistance of the transgenic and non-transgenic host with the host's oxylipin profile might additional strengthen the proposed role of Psi-factors in pathogenicity. 


\section{EXPERIMENTAL PART}

\subsection{Wet lab methods}

All bacterial cultures were inoculated under sterile conditions in a Sterile hood Prett/ ${ }^{\circledR}-$ Telsta Bioll-A from Telstar (Terrassa, Spain) and all glassware and disposable tips were autoclaved prior to usage. Unless stated otherwise, all materials were purchased from Carl Roth (Karlsruhe, Germany) and of highest purity available.

\subsubsection{KITS AND MARKER}

Kits: Nucleospin ${ }^{\mathrm{TM}}$ plasmid kit from Machery\&Nagel (Düren, Germany)

Protein Marker: Protein molecular weight marker ${ }^{\mathrm{TM}}$ from Fermantas (St. Leon-Rot, Germany)

Enzymes: Pfu-DNA-Polymerase and Dpnl were obtained from Fermantas

\subsubsection{MEDIA AND ANTIBIOTIC}

Luria-Bertani (LB)-medium: Pepton: $10 \mathrm{~g} / \mathrm{l}$; yeast extract: $10 \mathrm{~g} / \mathrm{l} ; \mathrm{NaCl}: 5 \mathrm{~g} / \mathrm{l}$ in $\mathrm{diH}_{2} \mathrm{O}$; the medium was autoclaved at $120^{\circ} \mathrm{C}$ for $20 \mathrm{~min}$.

2*YT medium: Pepton: $30 \mathrm{~g} / \mathrm{l}$; yeast extract: $20 \mathrm{~g} / \mathrm{l}$; $\mathrm{NaCl}: 5 \mathrm{~g} / \mathrm{l}$ in $\mathrm{diH}_{2} \mathrm{O}$; the medium was autoclaved at $120{ }^{\circ} \mathrm{C}$ for $20 \mathrm{~min}$.

Antibiotic: Kanamycin was added to all cultivations to a final concentration of $50 \mu \mathrm{g} / \mathrm{ml}$

LB-Plates: For preparation of agar plates, $1.5 \%(w / v)$ agar was added to the medium.

\subsubsection{StRAINS}

Organism

Strain

Genotype

Source

\begin{tabular}{|c|c|c|c|}
\hline E. coli & XL1Blue & $\begin{array}{c}\text { recA1endA1gyrA96 thi- } \\
\text { 1hsdR17 supE44 } \\
\text { relA1lac[F'proAB lac9zM15 } \\
\text { Tn19(Tetr)] }\end{array}$ & $\begin{array}{c}\text { Invitrogen } \\
\text { (Darmstadt, } \\
\text { Germany) }\end{array}$ \\
\hline E. coli & BL21* & $\begin{array}{c}\text { F-ompT hsdSB }\left(r_{B}-m_{B}\right) \text { gal } \\
\text { dcm araB:T7RNAP-tetA }\end{array}$ & $\begin{array}{c}\text { Invitrogen } \\
\text { (Darmstadt, } \\
\text { Germany) }\end{array}$ \\
\hline
\end{tabular}

Table 6: Used organisms and strains. 


\subsubsection{VECTORS}

PpoA was expressed from pET24a plasmid (Merck, Darmstadt, Germany) as described in literature (Brodhun et al., 2009).

\subsubsection{PREPARATION OF COMPETENT E. COLI FOR TRANSFORMATION BY HEAT-SHOCK}

$300 \mathrm{ml}$ LB-Medium was inoculated with $300 \mu \mathrm{l}$ of an overnight culture of the respective E.coli strain and grown at $37{ }^{\circ} \mathrm{C}$ to an $\mathrm{OD}_{600} \sim 0.5$. The cell suspension was chilled for $10 \mathrm{~min}$ on ice and cells were subsequently harvested by centrifugation ( $20 \mathrm{~min} ; 3000 * \mathrm{~g})$. The pellet was washed once with $100 \mathrm{ml}$ TFB-buffer and cells were resuspended in $16 \mathrm{ml}$ TFB-buffer and $1.2 \mathrm{ml}$ DMSO. $300 \mu \mathrm{l}$ aliquots were prepared and shock-frozen in liquid nitrogen. Until use, they were stored at $-80^{\circ} \mathrm{C}$.

\section{TB-Buffer:}

$10 \mathrm{mM}$ PIPES pH $6.7(\mathrm{KOH})$, sterile filtered

$55 \mathrm{mM} \mathrm{MnCl}_{2}$

$15 \mathrm{mM} \mathrm{CaCl}_{2}$

$250 \mathrm{mM} \mathrm{KCl}$

\subsubsection{TRANSFORMATION}

For transformation of the plasmids in E. coli the competent cells were thawed on ice. Subsequently, $100 \mu \mathrm{l}$ of the cell suspension were added to the site-directed mutation reaction or $2 \mu \mathrm{l}$ of plasmidDNA. This mixture was incubated for 20 min on ice. Afterwards, the cells were heat-shocked in a water bath for $45 \mathrm{sec}$ at $42{ }^{\circ} \mathrm{C}$ and instantly cooled on ice for $5 \mathrm{~min}$. After addition of $900 \mu \mathrm{LB}-$ medium the cells were shaken at $200 \mathrm{rpm}$ for $90 \mathrm{~min}$ at $37{ }^{\circ} \mathrm{C}$. Transformands were selected by spreading this cell suspension on LB-agar-plates containing $50 \mu \mathrm{g} / \mathrm{ml}$ kanamycin. The plates were incubated over night at $37 \mathrm{C}$ and single colonies were picked for subsequent incubations.

\subsubsection{PLASMID-DNA-ISOLATION FROM E. COLI}

For isolation of plasmid-DNA $5 \mathrm{ml} \mathrm{LB}$-medium containing $50 \mathrm{\mu g} / \mathrm{ml}$ Kanamycin was inoculated with a single bacterial colony and cultivated over night at $37^{\circ} \mathrm{C}$ and $200 \mathrm{rpm}$. Plasmid preparation from this culture was facilitated by the Nucleospin ${ }^{\mathrm{TM}}$ Plasmid Kit according to the manufacturer's protocol. Deviating from this protocol, elution of DNA was performed by addition of $50 \mu \mathrm{lim}_{2} \mathrm{O}$. Plasmid-DNA was stored at $-20^{\circ} \mathrm{C}$. 


\subsubsection{Site-directed mutagenesis by Polymerase-Chain-Reaction}

Site-specific nucleotide exchanges were conducted on the previously described expression plasmid (Brodhun et al., 2009) by utilization of oligonucleotides with specific mismatches at the desired position (Table 7). PCR amplification was performed by Pfu-Polymerase according to manufacturer's instructions on a Mastercyler from Eppendorf AG (Hamburg, Germany). After 25 cycles of PCR, template DNA was digested with Dpnl, precipitated and the resolved mutated plasmid was transformed to XL1-Blue (Invitrogen, Darmstadt, Germany). Site-specificity of the conducted mutation was confirmed by sequencing of the entire open reading frame.

\subsubsection{DNA-SEQUENCING}

DNA-sequencing was performed using the Dye-Terminator-Cycle-Sequencing-Kit from Applied Biosystems (Carlsbad, USA). During DNA-synthesis, ddNTP's are randomly introduced, which leads to a nucleotide specific truncation of the strand. The ddNTP'S are labeled with distinct fluorescent dyes and the fragments are detected by their $3^{\prime}$-terminal nucleotide. Therefore, the mixture of the DNA strands of different lengths is separated by capillary electrophoresis, the fluorescent dye molecules are excited by a laser and fluorescence is detected by a photo cell. The sequencing reaction was performed as specified by Applied Biosystems with $1 \mu \mathrm{l}$ of plasmid and $2 \mu \mathrm{M}$ of the sequence specific oligonucleotide (Table 8). For purification sodium acetate ( $3 \mathrm{M}$ ), ethanol and EDTA (125 mM) were added, the mixture was stirred and then incubated for $5 \mathrm{~min}$. Subsequently, the mixture was centrifuged at $20000^{*} \mathrm{~g}$ and $4{ }^{\circ} \mathrm{C}$ for $15 \mathrm{~min}$. The resulting pellet was washed with ethanol $(70 \%)$, dried and dissolved in formamide $(15 \mu \mathrm{L})$. Acrylamide based electrophoretic separation of the specifically terminated DNA-fragments was done by the group of Prof. Pieler at GZMB, Goettingen. 


\begin{tabular}{|c|c|}
\hline Oligonucleotide name/ Mutation & Sequence \\
\hline Tyr327Phe_a & AAGATTTTGTCCGAACGATTTTGAATATAAACCGG \\
\hline Tyr327Phe_b & CGGACAAAATCTTTTTAGGATAATATTTGCG \\
\hline Tyr327Leu_a & TTATTTTAAAAGATTTGGTCCGAACGATTTTG \\
\hline Tyr327Leu_b & TTCGGACCAAATCTTTTAAAAATAATATTTGCG \\
\hline Tyr327Gln_a & TTATTTTAAAAGATCAGGTCCGAACGATTTTG \\
\hline Tyr327Gln_b & TTCGGACCTGATCTTTTTAAAATAATATTTGCG \\
\hline Arg336Met_a & GAATATAAACATGACAGATAGCACCTGGAGTTTGG \\
\hline Arg336Met_b & GCTATCTGTCATGTTTATATTCAAAATCGTTCGG \\
\hline Met791Leu_a & AGATCTTGGCTGCAGTTTTCACTGCC \\
\hline Met791Leu_b & TGCAGCCAAGATCTTGTACAGTTCCG \\
\hline Met791Leu/Ala792Val_a & CAAGATCTTGGTTGCAGTTTTCACTGCC \\
\hline Met791Leu/Ala792Val_b & TGCAACCAAGATCTTGTACAGTTCCG \\
\hline Met791Leu/Ala792Met_a & CAAGATCTTGATGGCAGTTTTCACTGCC \\
\hline Met791Leu/Ala792Met_b & TGCCATCAAGATCTTGTACAGTTCCG \\
\hline Phe795Leu_a & CAGTACTCACTGCCATCTTCTACGACGC \\
\hline Phe795Leu_b & ATGGCAGTGAGTACTGCAGCCATTATC \\
\hline Phe799Leu_a & GCCATCTTGTACGACGCAGATATTGGG \\
\hline Phe799Leu_b & TCGTACAAGATGGCAGTGAAAACTGC \\
\hline Phe799Tyr_a & CTGCCATCTACTACGACGCAGATATTGGG \\
\hline Phe799Tyr_b & GCGTCGTAGTAGATGGCAGTGAAAACTGC \\
\hline Phe799Trp_a & CTGCCATATGGTACGACGCAGATATTGGG \\
\hline Phe799Trp_b & GCGTCGTACCATATGGCAGTGAAAACTGC \\
\hline Phe799Met_a & CTGCCATCATGTACGACGCAGATATTGGG \\
\hline Phe799Met_b & GCGTCGTACATGATGGCAGTGAAAACTGC \\
\hline Asn887Val_a & AATGGTGGCAGTCCAAGCACAATTGTTTTCGC \\
\hline Asn887Val_b & GCGAAAACAATTGTGCTTGGACTGCCACCATT \\
\hline Leu1009Glu_a & GCGAAGATCTATGCAAGACAGG \\
\hline Leu1009Glu_b & GATCTTCGCCCAAACACTTGTGG \\
\hline
\end{tabular}

Table 7: Oligonucleotides, used for site-directed mutagenesis

\begin{tabular}{cc}
\hline Oligonucleotide name & Sequence \\
\hline T7-Promoter & TAATACGACTCACTATAGG \\
\hline PpoA_Seq_A & GACCTATTCCAGACAGACCC \\
\hline PpoA_Seq_B & ATGCAAGATTTTGTCGCGGG \\
\hline PpoA_Seq_C & TACAAGCTGGTTCTTCGCGC \\
\hline PpoA_Seq_D & GTTCGAGCTAAACCAGGCCG \\
\hline
\end{tabular}

Table 8: Oligonucleotides, used for sequencing 


\subsubsection{EXPRESSION OF PPOA WILD TYPE AND ITS VARIANTS IN $E$. COLI}

For expression of the recombinant protein, the respective plasmids were transformed in Bl21*. As preparatory cultures LB-medium containing $50 \mu \mathrm{g} / \mathrm{ml}$ Kanamycin was inoculated with one transgenic colony and shaken at $200 \mathrm{rpm}$ over night at $37^{\circ} \mathrm{C}$. These preparatory cultures were used to inoculate the expression cultures ( 1 I in 2 I shaking flasks; $2^{*} Y T$ with $50 \mu \mathrm{g} / \mathrm{ml}$ Kanamycin). The cultures were cultivated until they reached an $\mathrm{OD}_{600}$ of $0.6-0.8$. Then a spatula green ammonium ferric citrate as well as a spatula aminolevulinic acid (Sigma, Steinheim, Germany) was added and expression was induced by addition of IPTG to a final concentration of $0.1 \mathrm{mM}$. Subsequently the cultures were further cultivated for $24 \mathrm{~h}$ at $28^{\circ} \mathrm{C}$. The cells were harvested by centrifugation at $8000^{*} \mathrm{~g}$ at $4{ }^{\circ} \mathrm{C}$ for $20 \mathrm{~min}$. The cell pellet was dissolved in lysis buffer and this suspension was stored at $-20^{\circ} \mathrm{C}$.

\section{Lysis buffer:}

$50 \mathrm{mM}$ TRIS, $\mathrm{pH} 7.4$

$10 \%(v / v)$ Glycerol

5 mM EDTA

Lysozyme (spatula tip)

\subsubsection{CELL DISRUPTION OF E. COLI EXPRESSION CULTURES}

The dissolved cell pellet was thawed on ice. To further facilitate cell disruption, pulsed sonication $(5 \mathrm{x}$ $45 \mathrm{sec}, 50 \%$ power, 50 \% impulse) was performed with a Sonoplus GM 7 from Bandelin (Berlin, Germany). The cell debris was removed by centrifugation at $50.000 * \mathrm{~g}$ at $4^{\circ} \mathrm{C}$ for $20 \mathrm{~min}$ and the clarified supernatant was poured and used for further purification.

\subsubsection{ENZYME PURIFICATION}

Enzyme was purified as described previously (Brodhun et al., 2009). Briefly, cell pellet was resuspended in lysis-buffer (50 mM Tris-buffer, pH 7.5/ 5 mM EDTA/ $10 \%(v / v)$ glycerol) and after cell disruption by lysozyme-treatment and ultrasonication a two-step chromatographical sequence was utilized with a strong anion exchanger for enzyme capture (Source 30Q (GE-healthcare, Freiburg, Germany) with $50 \mathrm{mM}$ Tris-buffer, $\mathrm{pH} 7.5$, and $5 \mathrm{mM} \mathrm{EDTA}$; $\mathrm{dV} / \mathrm{dt}=2 \mathrm{ml} / \mathrm{min}$; elution with a linear gradient from 0 to $300 \mathrm{mM} \mathrm{NaCl}$ over $25 \mathrm{~min}$ ) and a size exclusion column (Superdex S200 26/60 (GEhealthcare, Freiburg, Germany) with a $20 \mathrm{mM}$ NaHEPES-buffer, $\mathrm{pH} 7.4 ; \mathrm{dV} / \mathrm{dt}=2 \mathrm{ml} / \mathrm{min}$ ) for polishing of the enzyme.

\subsubsection{SDS-POLLYACRYLAMIDE-GEL ELLEKTROPHORESIS (SDS-PAGE)}

SDS-PAGE were cast and run on a Mini-PROTEAN3 Electrophoresis System from Bio-Rad Laboratories $\mathrm{GmbH}$ (Muenchen, Germany). The samples were mixed with FIB's Laemmli-buffer (Seesen, Germany) and boiled for $5 \mathrm{~min}$. Typically, $10 \mu \mathrm{l}$ of the denatured samples were loaded into the wells of the stacking gel and the gel was run with $30 \mathrm{~mA}$ until the bromphenol front was not visible anymore. Proteins were stained overnight by Coomassie Brilliant Blue and destained with water. Optionally, 
protein-bands were excised and identified by ESI-MS. The latter was done by Dr. Oliver Valerius, Institute for Microbiology and Genetics, Georg-August-University, Goettingen.

Stacking gel $(4.8 \%)$

$0.5 \mathrm{M}$ Tris/ $\mathrm{HCl}, \mathrm{pH}=6.7 ; 0,4 \%(w / v)$ SDS

$375 \mu l$

$30 \%$ Acrylamide/Bisacrylamide $(12.5: 1, w / v) \quad 240 \mu \mathrm{l}$

$25 \%(w / v)$ APS $\quad 3 \mu \mathrm{l}$

TEMED $\quad 6 \mu l$

$\mathrm{diH}_{2} \mathrm{O} \quad 885 \mu \mathrm{l}$

\section{Seperation gel $(10 \%)$}

$1.5 \mathrm{M}$ Tris $/ \mathrm{HCl}, \mathrm{pH}=8.8 ; 0,4 \%$ SDS

$1000 \mu \mathrm{l}$

$30 \%$ Acrylamide/Bisacrylamide $(12.5: 1, w / v)$

$1330 \mu \mathrm{l}$

$25 \%(w / v)$ APS

$3 \mu \mathrm{l}$

TEMED

$6 \mu \mathrm{l}$

$\mathrm{diH}_{2} \mathrm{O}$

$1670 \mu \mathrm{l}$

\section{SDS-PAGE-buffer}

$25 \mathrm{mM}$ Tris $/ \mathrm{HCl}, \mathrm{pH}=8.0$

$200 \mathrm{mM}$ glycerine

$0.1 \%(w / v)$ SDS

\section{Coomassie-staining solution}

$40 \%(v / v)$ Methanol

$10 \%(v / v)$ Acetic acid

Coomassie Brilliant Blue G250

\subsubsection{UV-VIS AND CD-SPECTROSCOPY}

UV-Vis spectra were recorded against a matching buffer on a Cary 100 Bio UV-visible spectrophotometer from Varian (Palo Alto, USA). The enzyme was diluted to an appropriate concentration $(<1 \mathrm{~g} / \mathrm{l})$ and measured in a $10 \mathrm{~mm}$ quartz cuvette. Enzyme-concentrations were determined utilizing the theoretically calculated specific extinction coefficient $\varepsilon_{280}=125500 \mathrm{M}^{-1} \mathrm{~cm}^{-1}$.

CD-spectra were recorded on a Chirascan from Applied Photophysics (Leatherhead, UK) against 20 $\mathrm{mM}$ HEPES-buffer, $\mathrm{pH}$ 7.4. The temperature was fixed at $20^{\circ} \mathrm{C}$ and the enzyme concentration was adjusted to $1.2 \mathrm{mg} / \mathrm{ml}$. For far-UV and Vis-CD spectra a $10 \mathrm{~mm}$ cuvette was used, while near-UV CDspectra were measured with enzyme placed in a $1 \mathrm{~mm}$ cuvette.

\subsubsection{Tryptic digestion of native PpoA}

For digestion of PpoA purified enzyme in $20 \mathrm{mM}$ HEPES, pH 7.4, was treated with $1: 200(\mathrm{w} / \mathrm{w})$ trypsin (Sigma; Steinheim; Germany). This mixture was incubated over night at $4{ }^{\circ} \mathrm{C}$. For removal of 
the protease, size exclusion chromatography was utilized as outlined in section 8.1.12. Alternatively the digested protein was directly analyzed by SDS-PAGE.

\subsubsection{GRAFIX}

The method for fixation of a certain oligomeric state within a density gradient was applied with modifications from literature (Kastner et al., 2008). For PpoA not a continuous, but a discontinuous saccharose gradient with a discontinuous glutaraldehyde gradient in the opposite orientation was used. The gradient was prepared in 50 mM HEPES, pH 7.5, and the gradient concentrations were adjusted as outlined in figure 12. The gradient was prepared in $12 \mathrm{ml}$ tubes and PpoA was applied as thin, concentrated band on top of the gradient. Separation of the applied sample and concomitant fixation of a certain oligomeric state was accomplished by centrifugation $250000^{*} \mathrm{~g}$ at and $4{ }^{\circ} \mathrm{C}$ for 48 h. Using this protocol both, untreated and previously trypsin digested PpoA, were cross-linked.

\subsubsection{REDUCTIVE METHYLATION OF SURFACE EXPOSED LYSINES}

Reductive methylation was carried out as described in literature (Kim et al., 2008). For this kind of protein modification PpoA at a concentration of $7 \mathrm{~g} / \mathrm{l}$ in $50 \mathrm{mM}$ HEPES, pH 8.0/500 mM NaCl/ $5 \%$ $(v / v)$ glycerol/ $10 \mathrm{mM}$ ß-mercaptoethanol was used. Alternatively also trypsin treated PpoA (section 8.1.15) was used for this modification. Dimethylamine-borane (Sigma, Steinheim, Germany) was solved in water to a final concentration of $1 \mathrm{M}$ and $1 \mathrm{M}$ formaldehyde was prepared by boiling 300

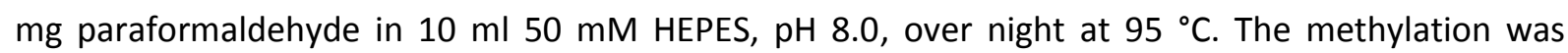
accomplished by addition of $80 \mu \mathrm{l} 1 \mathrm{M}$ formaldehyde and $40 \mu \mathrm{l} 1 \mathrm{M}$ dimethylamine-borane to $2 \mathrm{ml}$ of the protein. This mixture was incubated on ice for $2 \mathrm{~h}$ and the addition of methylating reagents was repeated two times. Upon incubation over night, the reaction was stopped by addition of glycine to a final concentration of $13.3 \mathrm{mM}$ and DTT to a final concentration of $5 \mathrm{mM}$. Size exclusion chromatography was applied to exchange the buffer and remove the reagents.

\subsubsection{MEASURING DOX-KINETICS BY MONITORING DEPLETION OF DISSOLVED OXYGEN}

Continuous DOX-kinetics were measured by monitoring oxygen depletion during the reaction of 100 to $500 \mathrm{nM}$ Ppo-enzyme with various fatty acids in different concentrations. The reactions were conducted in $20 \mathrm{mM}$ Na-HEPES, pH 7.5, and the temperature was adjusted with a water bath. Unless stated otherwise, the temperature was fixed at $27^{\circ} \mathrm{C}$. The respective substrate was prepared as $100-$ fold EthOH stock solution and $10 \mu \mathrm{l}$ of this stock were diluted in $980 \mu \mathrm{l}$ buffer. Dissolved oxygen in the reaction was measured using an Oxygraph (a Clark-type electrode) from Hansatech Instruments (Norfolk, UK) and the reaction was started by addition of enzyme. For evaluation, velocity of dioxygenation was calculated by determination of the maximal slope of oxygen depletion and assuming a stoichiometric, equimolar reaction of oxygen with fatty acid. The measured signal was correlated with the dissolved oxygen level by calibrating the system with sodium dithionite. Therefore, in a completely oxygen-saturated buffer solution, all dissolved oxygen was reduced by addition of sodium dithionite. The amplitude of the resulting voltage decrease was equalized to the total dissolved oxygen amount at the respective temperature, as they are tabulated in literature. 


\subsubsection{Synthesis of ${ }^{14}$ C-LABeled 8-HPODE AND 8-HPODE MEthyl ESTER}

$10 \mu \mathrm{l}$ of $100 \mu \mathrm{Ci} \cdot \mathrm{ml}^{-1} 1{ }^{14} \mathrm{C}_{1}$-linoleic acid (Perkin Elmer, Waltham, USA) and $5 \mu \mathrm{l}$ of $500 \mathrm{~g}^{*} \mathrm{I}^{-1}$ peroxidefree linoleic acid (Cayman, Ann Arbor, USA) were mixed in $400 \mu \mathrm{l}$ methanol and converted to methylated linoleic acid by addition of $10 \mu \mathrm{l} 2 \mathrm{M}$ trimethylsilyl-diazomethane in hexane (SigmaAldrich, Steinheim, Germany). The reaction was accomplished on a shaker at room temperature for $30 \mathrm{~min}$. After evaporation of the solvent under a gentle nitrogen stream, linoleic acid methyl ester was separated from the remaining free fatty acid by RP-HPLC. HPLC conditions were chosen identical to those described by Brodhun et al. (Brodhun et al., 2009). ${ }^{14} \mathrm{C}$-labeled 8-HPODE was produced by conversion of $10 \mu \mathrm{L}$ of $100 \mu \mathrm{Ci} \cdot \mathrm{ml}^{-1} 1_{-14} \mathrm{C}_{1}$-linoleic acid and $5 \mu \mathrm{l}$ of $500 \mathrm{~g}^{*} \mathrm{I}^{-1}$ peroxide-free linoleic acid by PpoA-His1004Ala (Brodhun et al., 2009). The fatty acid peroxide product was isolated by RP-HPLC and subsequently the corresponding methyl ester was furnished by reaction with trimethylsilyldiazomethan. The methylated 8-HPODE was again isolated by RP-HPLC. These substrate methyl esters were finally converted with $100 \mathrm{nM}$ of the respective enzyme in $20 \mathrm{mM} \mathrm{HEPES}, \mathrm{pH} 7.4$, for 30 min at room temperature. After extraction with diethyl ether, the conversion products were analyzed by RP-HPLC coupled to a Ramona Star scintillation counter (Raytest, Straubenhardt, Germany).

\subsubsection{SUBStRATE CONVERSION AND ANALYSIS OF THE PRODUCT PATTERN BY LC-MS ${ }^{2}$}

Reactions were typically carried out in Na-HEPES $(20 \mathrm{mM}, \mathrm{pH}$ 7.5). The reaction buffer was prepared by dilution of the 100-fold substrate working concentration (10 $\mu \mathrm{l}$; prepared in EtOH) in buffer (980 $\mu l)$. Enzymatic conversion was started by mixing this buffer with PpoA. Subsequently, the reaction was shaken for two minutes and stopped afterwards by extraction with $\mathrm{Et}_{2} \mathrm{O}$. The solvent was evaporated under a $\mathrm{N}_{2}$-stream and the products were redissolved in $\mathrm{MeCN} / \mathrm{H}_{2} \mathrm{O} / \mathrm{HAc}$ (50: $50: 0.1$ $(\mathrm{v} / \mathrm{v})$ ). LC/MS ${ }^{2}$ was performed as described previously (Brodhun et al., 2009). The relative product distribution was determined by relation of the respective product signal-intensity to the intensitysum of all products formed. Therefore, the single products were identified by $\mathrm{MS}^{2}$. Both, the use of deuterium labels at different sites of the fatty acid backbone as well as conversion of the substrate's ${ }^{13} \mathrm{C}_{18}$-isotopologue, allowed a reliable identification of the single fragments and facilitated the identification of the products formed. The signals of the distinct products were integrated after extracting the molecular weight of the product from the total ion count of the MS. To calculate the molecular weight of the products, deprotonation of the carboxylic group and isotopologues of the substrate have to be considered.

\subsubsection{Measurement of tyrosyl Radical distances by DEER}

CW-EPR spectra were recorded on a Bruker EMX spectrometer equipped with a Bruker ER041 bridge. The resonator was cooled by a liquid helium cryostat from Oxford Instruments. Detailed parameters of the measurement are specified elsewhere (Fielding et al., 2011).

Pulsed EPR-measurements were perfomed on a Bruker ELEXSYS E580. Temperature was adjusted using a liquid helium cryostat CF935 from Oxford Instruments. For highest sensitivity, the 
measurements were performed at $6 \mathrm{~K}$ (determination of relaxation times and echo detected field sweeps) and $5 \mathrm{~K}$ (four-pulse DEER), respectively. Measurement of $\mathrm{T}_{1}$ was done utilizing an inversion recovery sequence: $\pi-\tau_{1}-\pi / 2-\tau_{2}-\pi-\tau_{2}$-detect, with $\tau_{1}$ being increased. The time window of this experiment was adjusted to a length that covered a complete saturation of the signal. $T_{m}$ was measured with a two-pulse echo decay experiment: $\pi / 2-\tau_{1}-\pi-\tau_{1}-$ detect, with $\tau_{1}$ being increased. Depending on the relaxation time, the time window was adjusted to cover the whole exponential decay. DEER data were collected utilizing the dead-time free four-pulse sequence presented in section 3.4.1 (Pannier et al., 2000). Distance distributions were extracted from dipolar time evolution data using Tikhonov regularization as implemented in DeerAnalysis2011 (G. Jeschke et al., 2006).

\subsubsection{Direct electrochemistry of PpoA Within a SURfactant film}

A self assembled DDAB-film was established on an electrode made of pyrolytic graphite by air evaporating $3 * 10 \mu \mathrm{l}$ of a DDAB-solution (0.1 M in chloroform) on the electrode. Subsequently, PpoA wild type enzyme was bound to this surfactant film by equilibration of the electrode in a $25 \mu \mathrm{M}$ PpoA-solution (in $20 \mathrm{mM}$ HEPES, pH 7.4) for one week at $4{ }^{\circ} \mathrm{C}$. Enzyme integrity after this incubation was checked by UV/Vis-spectroscopy of the enzyme solution and conversion of linoleic acid. The electrode itself was utilized as working electrode to measure cyclic and square wave voltammograms. In both cases Ag was utilized as reference electrode, a Pt-wire served as counterelectrode and $50 \mathrm{mM} \mathrm{NaBr}$ in $50 \mathrm{mM} \mathrm{Na} \mathrm{HPO}_{4}, \mathrm{pH}$ 7.4, was utilized as electrolyte. Cyclic voltammograms were obtained in a range from $-1 \mathrm{~V}$ to $0.8 \mathrm{~V}$ while the scan-rate was varied between 10 and $50 \mathrm{mV} / \mathrm{sec}$. Square wave voltammograms were obtained in a range between -1 and $0 \mathrm{~V}$.

\subsubsection{IMIDAZOLE TITRATION AND SPECTRAL BINDING ASSAY}

Spectral binding assays were performed as described in literature (Yeh et al., 2005). Therefore, the enzyme solution ( $1 \mathrm{mg} / \mathrm{ml}$ in $20 \mathrm{mM}$ HEPES, $\mathrm{pH} 7.5$ ) was titrated with a concentrated imidazole stock solution. The vis-spectrum was recorded upon each imidazole addition. For evaluation the difference-spectrum was calculated and the peak-trough difference was plotted against the imidazole concentration. Dissociation constants were calculated assuming either a one-site $\left((A 417-A 402)=\frac{B m a x *[\text { imidazole }]}{k D+[\text { imidazole }]}\right) \quad$ or $\quad$ a $\quad$ two-site $\quad\left((A 417-A 402)=\frac{B \text { max } 1 *[\text { imidazole }]}{k D 1+[\text { imidazole }]}+\right.$ $\left.\frac{B m a x 2 *[\text { imidazole }]}{k D 2+[\text { imidazole }]}\right)$ binding model. 


\subsection{Bioinformatic methods}

Molecular weight and $\varepsilon_{280}$ of PpoA were calculated using the ProtParam tool from Swissprot (http://web.expasy.org/protparam/).

For template-based structure prediction several algorithms have been tested (e.g. http://raptorx.uchicago.edu/; http://casp.rnet.missouri.edu/multicom_3d.html), but finally I-TASSER (http://zhanglab.ccmb.med.umich.edu/I-TASSER/) was chosen.

Chimera 1.5.3 was obtained from University of California, San Francisco (http://www.cgl.ucsf.edu/chimera/download.html) and utilized for molecular modeling as well as rendering. Raytracing was performed with POV-Ray (http://www.povray.org/).

Amino acid identities and similarities were calculated using the web interface SIAS (http://imed.med.ucm.es/Tools/sias.html).

Sequence alignments and analysis was done using the BioEdit package from Ibis Biosciences (http://www.mbio.ncsu.edu/bioedit/bioedit.html).

2D Plots of active site residues were calculated with LigPlot ${ }^{+}$(http://www.ebi.ac.uk/thorntonsrv/software/LigPlus/) (Laskowski and Swindells, 2011). 


\section{ACKNOWLEDGEMENT}

I am just a dwarf standing on the shoulder of giants

-Bernhard von Chartres

This thesis emerged from my work in the department of Plant Biochemistry at the Albrecht-vonHaller Institute, Georg-August-University Goettingen. I am indebted to Prof. Ivo Feußner who supervised my work and supported me in all kinds of scientific life and questions. At the same time he allowed me to evolve my own ideas and supported them to the end of learning a truly autonomous kind of research. The most outstanding support to this work was laid by Dr. Florian Brodhun, who established PpoA in Prof. Feußner's working group and thus was one of the most important contact persons in my daily scientific life. I am also grateful for the help of Benjamin Goede, who disburdened me in daily lab routine for half a year. For expertise purification of 8-HPODE as crucial substrate for rearrangement reactions and enzyme activation, the work of Sabine Freitag is greatly acknowledged. The support with and establishment of oxylipin analytics by Dr. Cornelia Herrfurth is acknowledged. Although without successful results, I am also indebted to Dr. Ellen Hornung and Susanne Mester, who helped me with physiological studies on the effect of Ppoenzymes on plants. Despite their sacrificially efforts my plant-physiological experiments were unfortunately governed by my underdeveloped green thumb. Additionally, I am thankful to Ellen for filling up my physical energy reservoirs in a tasty way. Last but not least, I want to express my gratitude to Gerhard Marder. His steady engagement in order to maintain the lab equipment was an essential input to keep the experiments running. I also want to express my thanks to Florian and Prof. Feußner, for careful reading the manuscript of this thesis and supporting its writing with valuable comments.

Besides the whole department of Plant Biochemistry, several collaborators supported this work. Dr. Andre Nadler and Prof. Ulf Diederichsen from the Institute of Organic and Biomolecular Chemistry synthesized specifically deuterated oleic acids for measurement of KIEs and greatly assisted in interpreting the obtained data and writing the manuscript for my very first publication, a rememberable step in my scientific career. Also from the Department of Bioanalytics valuable support to this thesis was given. Dr. Danilo Meyer helped me with CD-spectroscopy and Dr. Kathrin Schroeder-Tittmann enabled me to perform a Pichia pastoris fermentation and express a PpoA homologue. Also the head of the Department, Prof. Kai Tittmann, gave valuable input to this thesis by helping with the interpretation of KIE-data and inspiring advises during our thesis committee meetings. Small angle $\mathrm{X}$-ray scattering data, were obtained at the $\mathrm{X}-33$ beamline at the DESY in Hamburg. Therefore, I am grateful to the BioSAXS-group of Prof. Dimitri Svergun, and especially the help of Giancarlo Tria in obtaining and interpreting the data is emphasized here. In this context, I also want to express my gratitude to Dr. Oliver Valerius (Department of Molecular Microbiology and Genetics, University Goettingen), who determined exposed trypsin cleavage sites in the native 
enzyme by ESI-MS. The attempts to crystallize PpoA were conducted in the group of Prof. Ficner and the practical help of Michael Franke and Daniel Weinrich are acknowledged. I am especially indebted to Dr. Piotr Neumann, who gave me advice of how to proceed and, most important, encouraged me, whenever the experiments did not work as they should. DNA-sequencing was performed in the group of Prof. Pieler (Department of Developmental Biochemistry, University Goettingen) by Andreas Nolte.

I want to emphasize especially our collaboration with the EPR-group of Prof. Marina Bennati at the MPI for biophysical chemistry in Goettingen. Not only her advices during our thesis committee meetings were a source of inspiration, but together we could elucidate some important features of the intriguing mechanism of lipid oxidation catalyzed by PpoA and she gave me the chance to perform the DEER-meausrements on my own. The work of Dr. Alistair Fielding and Dr. Roberta Pievo and our stimulating discussions enabled me to get some important insights into how EPR spectroscopy works. I also want to thank the whole group for the support they gave me during my own measurements of tyrosyl radical distances, and emphasize the contributions of Dr. Giuseppe Sicoli and especially the advice, guidance and the good portion of British humor given by Alistair.

The international research training group: "Metal Sites in Biomolecules: Structures, Regulation and Mechanisms" did not only financially support this thesis, but our regular meetings, workshop and symposia were a great source of inspiration for my work. I want to name especially the input of Antonia Albers, who enabled me to measure cyclic voltammograms in the group of Prof. Franc Meyer. Moreover, Prof. Derek Logan inspired me in one of our joint workshops with our Swedish colleagues that SAXS might be a suitable technique to address the quaternary structure of PpoA. 


\section{REFERENCES}

Andreou, A., F. Brodhun, and I. Feussner, 2009, Biosynthesis of oxylipins in non-mammals: Progress in Lipid Research, v. 48, no. 3-4, p. 148-170, doi:10.1016/j.plipres.2009.02.002.

Anfinsen, C. B., 1973, Principles that Govern the Folding of Protein Chains: Science, v. 181, no. 4096, p. $223-230$, doi:10.1126/science.181.4096.223.

Armstrong, F. A., 2009, Dynamic electrochemical experiments on hydrogenases: Photosynthesis Research, doi:10.1007/s11120-009-9428-0.

Bauler, P., G. Huber, T. Leyh, and J. A. McCammon, 2010, Channeling by Proximity: The Catalytic Advantages of Active Site Colocalization Using Brownian Dynamics: The Journal of Physical Chemistry Letters, v. 1, no. 9, p. 1332-1335, doi:10.1021/jz1002007.

Bergfors, T. M., 1999, Protein Crystalization: Techniques, Strategies, and Tips : A Laboratory Manual: TBS The Book Service Ltd.

Bhardwaj, N., R. V. Stahelin, R. E. Langlois, W. Cho, and H. Lu, 2006, Structural bioinformatics prediction of membrane-binding proteins: Journal of Molecular Biology, v. 359, no. 2, p. 486-495, doi:10.1016/j.jmb.2006.03.039.

Bornscheuer, U. T., G. W. Huisman, R. J. Kazlauskas, S. Lutz, J. C. Moore, and K. Robins, 2012, Engineering the third wave of biocatalysis: Nature, v. 485, no. 7397, p. 185-194, doi:10.1038/nature11117.

Brash, A. R., 2009, Mechanistic aspects of CYP74 allene oxide synthases and related cytochrome P450 enzymes: Phytochemistry, v. 70, no. 13-14, p. 1522-1531, doi:10.1016/j.phytochem.2009.08.005.

Brodhagen, M., D. I. Tsitsigiannis, E. Hornung, C. Goebel, I. Feussner, and Nancy P. Keller, 2007, Reciprocal oxylipin-mediated cross-talk in the Aspergillus-seed pathosystem: Molecular Microbiology, v. 67, no. 2, p. 378-391, doi:10.1111/j.1365-2958.2007.06045.x.

Brodhun, F., and I. Feussner, 2011, Oxylipins in fungi: The FEBS Journal, v. 278, no. 7, p. 1047-1063, doi:10.1111/j.1742-4658.2011.08027.x.

Brodhun, F., C. Göbel, E. Hornung, and I. Feussner, 2009, Identification of PpoA from Aspergillus nidulans as a Fusion Protein of a Fatty Acid Heme Dioxygenase/Peroxidase and a Cytochrome P450: The Journal of Biological Chemistry, v. 284, no. 18, p. 11792-11805, doi:10.1074/jbc.M809152200.

Brodhun, F., S. Schneider, C. Göbel, E. Hornung, and I. Feussner, 2010, PpoC from Aspergillus nidulans is a fusion protein with only one active haem: The Biochemical Journal, v. 425 , no. 3, p. 553-565, doi:10.1042/BJ20091096.

Brodowsky, I. D., M Hamberg, and E H Oliw, 1992, A linoleic acid (8R)-dioxygenase and hydroperoxide isomerase of the fungus Gaeumannomyces graminis. Biosynthesis of (8R)-hydroxylinoleic acid and (7S,8S)-dihydroxylinoleic acid from (8R)-hydroperoxylinoleic acid.: Journal of Biological Chemistry, v. 267 , no. 21 , p. $14738-14745$. 
Brown, S. H., J. B. Scott, J. Bhaheetharan, W. C. Sharpee, L. Milde, R. A. Wilson, and Nancy P Keller, 2009, Oxygenase coordination is required for morphological transition and the host-fungus interaction of Aspergillus flavus: Molecular Plant-Microbe Interactions: MPMI, v. 22, no. 7, p. 882894, doi:10.1094/MPMI-22-7-0882.

Brown, D. W., J. H. Yu, H. S. Kelkar, M. Fernandes, T. C. Nesbitt, N P Keller, T. H. Adams, and T. J. Leonard, 1996, Twenty-five coregulated transcripts define a sterigmatocystin gene cluster in Aspergillus nidulans.: Proceedings of the National Academy of Sciences of the United States of America, v. 93, no. 4, p. 1418-1422.

Burow, G. B., T. C. Nesbitt, J. Dunlap, and N. P. Keller, 1997, Seed Lipoxygenase Products Modulate Aspergillus Mycotoxin Biosynthesis: Molecular Plant-Microbe Interactions, v. 10, no. 3, p. 380-387, doi:10.1094/MPMI.1997.10.3.380.

Calvo, A. M., L. L. Hinze, H. W. Gardner, and N P Keller, 1999, Sporogenic effect of polyunsaturated fatty acids on development of Aspergillus spp: Applied and Environmental Microbiology, v. 65, no. 8, p. 3668-3673.

Champe, S P, and A. A. el-Zayat, 1989, Isolation of a sexual sporulation hormone from Aspergillus nidulans: Journal of Bacteriology, v. 171, no. 7, p. 3982-3988.

Champe, S P, P. Rao, and A. Chang, 1987, An endogenous inducer of sexual development in Aspergillus nidulans: Journal of General Microbiology, v. 133, no. 5, p. 1383-1387.

Cheng, Y., C. A. Chang, Z. Yu, Yongjie Zhang, M. Sun, T. S. Leyh, M. J. Holst, and J. A. McCammon, 2008, Diffusional Channeling in the Sulfate-Activating Complex: Combined Continuum Modeling and Coarse-Grained Brownian Dynamics Studies: Biophysical Journal, v. 95, no. 10, p. 4659-4667, doi:10.1529/biophysj.108.140038.

Chiang, C.-W., H.-C. Yeh, L.-H. Wang, and N.-L. Chan, 2006, Crystal Structure of the Human Prostacyclin Synthase: Journal of molecular biology, v. 364, no. 3, p. 266-274, doi:10.1016/j.jmb.2006.09.039.

Christensen, S. A., and M. V. Kolomiets, 2011, The lipid language of plant-fungal interactions: Fungal Genetics and Biology: FG \& B, v. 48, no. 1, p. 4-14, doi:10.1016/j.fgb.2010.05.005.

Conner, K. P., C. M. Woods, and W. M. Atkins, 2011, Interactions of cytochrome P450s with their ligands: Archives of Biochemistry and Biophysics, v. 507, no. 1, p. 56-65, doi:10.1016/j.abb.2010.10.006.

Cooper, D. R., T. Boczek, K. Grelewska, M. Pinkowska, M. Sikorska, M. Zawadzki, and Z. Derewenda, 2007, Protein crystallization by surface entropy reduction: optimization of the SER strategy: Acta Crystallographica. Section D, Biological Crystallography, v. 63, no. Pt 5, p. 636-645, doi:10.1107/S0907444907010931.

Coppock, R. W., and B. J. Jacobsen, Mycotoxins in animal and human patients: Toxicology and Industrial Health, v. 25, no. 9-10, p. $637-655$, doi:10.1177/0748233709348263. 
Correia, M. A., K. Q. Yao, A. J. Allentoff, S. A. Wrighton, and J. A. Thompson, 1995, Interactions of Peroxyquinols with Cytochromes P450 2B1, 3A1, and 3A5 - Influence of the Apoprotein on Heterolytic Versus Homolytic O-O Bond-Cleavage: Archives of Biochemistry and Biophysics, v. 317, no. 2, p. 471-478, doi:10.1006/abbi.1995.1190.

Corvaja, C., M. Bowman, M. Brustolon, and E. Giamello, 2009, Electron Paramagnetic Resonance (A practioner's Toolkit): New Jersey, John Wiley \& Sons, 3-36; 159-194 p.

Cristea, M., A. E. Osbourn, and Ernst H Oliw, 2003, Linoleate diol synthase of the rice blast fungus Magnaporthe grisea: Lipids, v. 38, no. 12, p. 1275-1280.

Daff, S. N., S. K. Chapman, R. A. Holt, S. Govindaraj, T. L. Poulos, and A. W. Munro, 1997, Redox Control of the Catalytic Cycle of Flavocytochrome P-450 BM3+: Biochemistry, v. 36, no. 45, p. 1381613823, doi:10.1021/bi971085s.

Daiyasu, H., and H. Toh, 2000, Molecular evolution of the myeloperoxidase family: Journal of Molecular Evolution, v. 51, no. 5, p. 433-445.

Danish, H. H., I. S. Doncheva, and J. P. Roth, 2011, Hydrogen Tunneling Steps in Cyclooxygenase-2 Catalysis: J. Am. Chem. Soc., v. 133, no. 40, p. 15846-15849, doi:10.1021/ja2059523.

Denisov, I. G., T. M. Makris, S. G. Sligar, and I. Schlichting, 2005, Structure and chemistry of cytochrome P450: Chemical Reviews, v. 105, no. 6, p. 2253-2277, doi:10.1021/cr0307143.

Derewenda, Z. S., 2004, Rational protein crystallization by mutational surface engineering: Structure (London, England: 1993), v. 12, no. 4, p. 529-535, doi:10.1016/j.str.2004.03.008.

Derewenda, Z. S., and P. G. Vekilov, 2006, Entropy and surface engineering in protein crystallization: Acta Crystallographica. Section D, Biological Crystallography, v. 62, no. Pt 1, p. 116-124, doi:10.1107/\$0907444905035237.

Dietz, R., W. Nastainczyk, and H. H. Ruf, 1988, Higher oxidation states of prostaglandin H synthase. Rapid electronic spectroscopy detected two spectral intermediates during the peroxidase reaction with prostaglandin G2: European Journal of Biochemistry / FEBS, v. 171, no. 1-2, p. 321-328.

Dong, L., A. J. Vecchio, N. P. Sharma, B. J. Jurban, M. G. Malkowski, and W. L. Smith, 2011, Human Cyclooxygenase-2 is a Sequence Homodimer That Functions as a Conformational Heterodimer: Journal of Biological Chemistry, v. 286, no. 21, p. 19035 -19046, doi:10.1074/jbc.M111.231969.

Fabbri, A. A., C. Fanelli, G. Panfili, S. Passi, and P. Fasella, 1983, Lipoperoxidation and Aflatoxin Biosynthesis by Aspergillus parasiticus and A. flavus: Journal of General Microbiology, v. 129, no. 11, p. 3447-3452, doi:10.1099/00221287-129-11-3447.

Fanelli, C, and A A Fabbri, 1989, Relationship between lipids and aflatoxin biosynthesis: Mycopathologia, v. 107, no. 2-3, p. 115-120.

Fielding, A. J., F. Brodhun, C. Koch, R. Pievo, V. P. Denysenkov, I. Feussner, and M. Bennati, 2011, Multifrequency Electron Paramagnetic Resonance Characterization of PpoA, a CYP450 Fusion Protein that Catalyses Fatty Acid Dioxygenation: Journal of the American Chemical Society, doi:10.1021/ja202207t. 
Fishelovitch, D., S. Shaik, H. J. Wolfson, and R. Nussinov, 2009, Theoretical Characterization of Substrate Access/Exit Channels in the Human Cytochrome P450 3A4 Enzyme: Involvement of Phenylalanine Residues in the Gating Mechanism: The Journal of Physical Chemistry. B, v. 113, no. 39, p. 13018-13025, doi:10.1021/jp810386z.

Förster, F., B. Webb, K. A. Krukenberg, H. Tsuruta, D. A. Agard, and A. Sali, 2008, Integration of small angle $\mathrm{X}$-ray scattering data into structural modeling of proteins and their assemblies: Journal of molecular biology, v. 382, no. 4, p. 1089-1106, doi:10.1016/j.jmb.2008.07.074.

Fowler, P. W., and P. V. Coveney, 2006, A computational protocol for the integration of the monotopic protein prostaglandin $\mathrm{H} 2$ synthase into a phospholipid bilayer: Biophysical Journal, v. 91, no. 2 , p. 401-410, doi:10.1529/biophysj.105.077784.

Franke, D., and D. I. Svergun, 2009, DAMMIF, a program for rapid ab-initio shape determination in small-angle scattering: Journal of Applied Crystallography, v. 42, no. 2, p. 342-346, doi:10.1107/S0021889809000338.

Franke, A., C. Fertinger, and R. van Eldik, 2008, Which Oxidant Is Really Responsible for P450 Model Oxygenation Reactions? A Kinetic Approach: Angewandte Chemie International Edition, v. 47, no. 28, p. 5238-5242, doi:10.1002/anie.200800907.

Frisvad, J. C., U. Thrane, R. A. Samson, and J. I. Pitt, 2006, Important mycotoxins and the fungi which produce them, in A. D. Hocking, J. I. Pitt, R. A. Samson, and U. Thrane, eds., Advances in Food Mycology: Boston, MA, Springer US, p. 3-31.

Fujishiro, T., O. Shoji, S. Nagano, H. Sugimoto, Y. Shiro, and Yoshihito Watanabe, 2011, Crystal Structure of H2O2-dependent Cytochrome P450SP $\alpha$ with Its Bound Fatty Acid Substrate: Journal of Biological Chemistry, v. 286, no. 34, p. 29941 -29950, doi:10.1074/jbc.M111.245225.

Funk, C. D., 2001, Prostaglandins and Leukotrienes: Advances in Eicosanoid Biology: Science, v. 294, no. 5548 , p. $1871-1875$, doi:10.1126/science.294.5548.1871.

Gao, X., M. Brodhagen, T. Isakeit, S. H. Brown, C. Göbel, J. Betran, I. Feussner, Nancy P Keller, and M. V. Kolomiets, 2009, Inactivation of the lipoxygenase ZmLOX3 increases susceptibility of maize to Aspergillus spp: Molecular Plant-Microbe Interactions: MPMI, v. 22, no. 2, p. 222-231, doi:10.1094/MPMI-22-2-0222.

Gao, X., W.-B. Shim, C. Göbel, S. Kunze, I. Feussner, R. Meeley, P. Balint-Kurti, and M. Kolomiets, 2007, Disruption of a maize 9-lipoxygenase results in increased resistance to fungal pathogens and reduced levels of contamination with mycotoxin fumonisin: Molecular Plant-Microbe Interactions: MPMI, v. 20, no. 8, p. 922-933, doi:10.1094/MPMI-20-8-0922.

Garscha, U., F. Jernerén, D. Chung, Nancy P Keller, Mats Hamberg, and Ernst H Oliw, 2007, Identification of dioxygenases required for Aspergillus development. Studies of products, stereochemistry, and the reaction mechanism: The Journal of Biological Chemistry, v. 282, no. 48, p. 34707-34718, doi:10.1074/jbc.M705366200.

Garscha, U., and Ernst H Oliw, 2008, Critical amino acids for the 8(R)-dioxygenase activity of linoleate diol synthase. A comparison with cyclooxygenases: FEBS Letters, v. 582, no. 23-24, p. 3547-3551, doi:10.1016/j.febslet.2008.09.031. 
Garscha, U., and Ernst H Oliw, 2009, Leucine/valine residues direct oxygenation of linoleic acid by (10R)- and (8R)-dioxygenases: expression and site-directed mutagenesis of (10R)-dioxygenase with epoxyalcohol synthase activity: The Journal of Biological Chemistry, v. 284, no. 20, p. 13755-13765, doi:10.1074/jbc.M808665200.

Garscha, U., and Ernst H Oliw, 2007, Steric analysis of 8-hydroxy- and 10-hydroxyoctadecadienoic acids and dihydroxyoctadecadienoic acids formed from 8R-hydroperoxyoctadecadienoic acid by hydroperoxide isomerases: Analytical Biochemistry, v. 367, no. 2, p. 238-246, doi:10.1016/j.ab.2007.04.045.

Ghimire, H., R. M. McCarrick, D. E. Budil, and G. A. Lorigan, 2009, Significantly improved sensitivity of Q-band PELDOR/DEER experiments relative to $X$-band is observed in measuring the inter-coil distance of a leucine zipper motif peptide (GCN4-LZ): Biochemistry, v. 48, no. 25, p. 5782-5784, doi:10.1021/bi900781u.

Goodrich-Tanrikulu, M., N. E. Mahoney, and S. B. Rodriguez, 1995, The plant growth regulator methyl jasmonate inhibits aflatoxin production by Aspergillus flavus: Microbiology (Reading, England), v. 141 ( Pt 11), p. 2831-2837.

Goodwin, D. C., S. W. Rowlinson, and L J Marnett, 2000, Substitution of tyrosine for the proximal histidine ligand to the heme of prostaglandin endoperoxide synthase 2: implications for the mechanism of cyclooxygenase activation and catalysis: Biochemistry, v. 39, no. 18, p. 5422-5432.

Gotoh, O., 1992, Substrate recognition sites in cytochrome P450 family 2 (CYP2) proteins inferred from comparative analyses of amino acid and coding nucleotide sequences: The Journal of Biological Chemistry, v. 267, no. 1, p. 83-90.

Green, M. T., J. H. Dawson, and H. B. Gray, 2004, Oxoiron(IV) in Chloroperoxidase Compound II Is Basic: Implications for P450 Chemistry: Science, v. 304, no. 5677, p. 1653 -1656, doi:10.1126/science.1096897.

Guengerich, F. P., 2002, Rate-limiting steps in cytochrome P450 catalysis: Biological Chemistry, v. 383 , no. 10, p. 1553-1564, doi:10.1515/BC.2002.175.

Gupta, A., A. Mukherjee, K. Matsui, and J. P. Roth, 2008a, Evidence for protein radical-mediated nuclear tunneling in fatty acid alpha-oxygenase: Journal of the American Chemical Society, v. 130, no. 34, p. 11274-11275, doi:10.1021/ja8042273.

Hamberg, M, L. Y. Zhang, I. D. Brodowsky, and E H Oliw, 1994, Sequential oxygenation of linoleic acid in the fungus Gaeumannomyces graminis: stereochemistry of dioxygenase and hydroperoxide isomerase reactions: Archives of Biochemistry and Biophysics, v. 309, no. 1, p. 77-80, doi:10.1006/abbi.1994.1087.

Hamberg, Mats, I. P. de León, A. Sanz, and C. Castresana, 2002, Fatty acid [alpha]-dioxygenases: Prostaglandins \& Other Lipid Mediators, v. 68-69, p. 363-374, doi:10.1016/S0090-6980(02)00040-0. Hamberg, Mats, and B. Samuelsson, 1967, On the Mechanism of the Biosynthesis of Prostaglandins E1 and F1 $\alpha$ : Journal of Biological Chemistry, v. 242, no. 22, p. $5336-5343$. 
Hamberg, Mats, A. Sanz, and C. Castresana, 2002, $\alpha$-Dioxygenase, a new enzyme in fatty acid metabolism: International Congress Series, v. 1233, no. 0, p. 307-317, doi:10.1016/S05315131(02)00158-9.

Hecker, M., and V. Ullrich, 1989, On the mechanism of prostacyclin and thromboxane A2 biosynthesis.: Journal of Biological Chemistry, v. 264, no. 1, p. $141-150$.

Hörnsten, L., Chao Su, A. E. Osbourn, P. Garosi, U. Hellman, C. Wernstedt, and Ernst H. Oliw, 1999, Cloning of Linoleate Diol Synthase Reveals Homology with Prostaglandin $\mathrm{H}$ Synthases: Journal of Biological Chemistry, v. 274, no. 40, p. $28219-28224$, doi:10.1074/jbc.274.40.28219.

Hu, N., 2001, Direct electrochemistry of redox proteins or enzymes at various film electrodes and their possible applications in monitoring some pollutants: Pure and Applied Chemistry, v. 73, no. 12, p. 1979-1991, doi:10.1351/pac200173121979.

Hunte, C., and H. Michel, 2002, Crystallisation of membrane proteins mediated by antibody fragments: Current Opinion in Structural Biology, v. 12, no. 4, p. 503-508.

Imai, M., H. Shimada, Y Watanabe, Y. Matsushima-Hibiya, R. Makino, H. Koga, T. Horiuchi, and Y. Ishimura, 1989, Uncoupling of the cytochrome P-450cam monooxygenase reaction by a single mutation, threonine-252 to alanine or valine: possible role of the hydroxy amino acid in oxygen activation: Proceedings of the National Academy of Sciences, v. 86, no. 20, p. $7823-7827$.

Jacques, D. A., and J. Trewhella, 2010, Small-angle scattering for structural biology-Expanding the frontier while avoiding the pitfalls: v. 19, no. 4, p. 642-657, doi:10.1002/pro.351.

Jernerén, F., A. Sesma, M. Franceschetti, M. Francheschetti, Mats Hamberg, and Ernst H Oliw, 2010, Gene deletion of 7,8-linoleate diol synthase of the rice blast fungus: studies on pathogenicity, stereochemistry, and oxygenation mechanisms: The Journal of Biological Chemistry, v. 285, no. 8, p. 5308-5316, doi:10.1074/jbc.M109.062810.

Jeschke, G., A. Bender, H. Paulsen, H. Zimmermann, and A. Godt, 2004, Sensitivity enhancement in pulse EPR distance measurements: Journal of Magnetic Resonance, v. 169, no. 1, p. 1-12, doi:10.1016/j.jmr.2004.03.024.

Jeschke, G., V. Chechik, P. Ionita, A. Godt, H. Zimmermann, J. Banham, C. Timmel, D. Hilger, and H. Jung, 2006, DeerAnalysis2006-a comprehensive software package for analyzing pulsed ELDOR data: Applied Magnetic Resonance, v. 30, no. 3, p. 473-498, doi:10.1007/BF03166213.

Jeschke, Gunnar, M. Sajid, M. Schulte, and Adelheid Godt, 2009, Three-spin correlations in double electron-electron resonance: Phys. Chem. Chem. Phys., v. 11, no. 31, p. 6580-6591, doi:10.1039/B905724B.

Johnson, D. L., C. J. Maxwell, D. Losic, J. G. Shapter, and L. L. Martin, 2002, The influence of promoter and of electrode material on the cyclic voltammetry of Pisum sativum plastocyanin: Bioelectrochemistry (Amsterdam, Netherlands), v. 58, no. 2, p. 137-147.

Karthein, R., R. Dietz, W. Nastainczyk, and H. H. Ruf, 1988, Higher oxidation states of prostaglandin H synthase. EPR study of a transient tyrosyl radical in the enzyme during the peroxidase reaction: European Journal of Biochemistry / FEBS, v. 171, no. 1-2, p. 313-320. 
Kastner, B. et al., 2008, GraFix: sample preparation for single-particle electron cryomicroscopy: Nature Methods, v. 5, no. 1, p. 53-55, doi:10.1038/nmeth1139.

Kim, Y. et al., 2008, Large-scale evaluation of protein reductive methylation for improving protein crystallization: Nature Methods, v. 5, no. 10, p. 853-854, doi:10.1038/nmeth1008-853.

Koszelak-Rosenblum, M., A. C. Krol, D. M. Simmons, C. C. Goulah, L. Wroblewski, and M. G. Malkowski, 2008, His-311 and Arg-559 Are Key Residues Involved in Fatty Acid Oxygenation in Pathogen-inducible Oxygenase: The Journal of Biological Chemistry, v. 283, no. 36, p. 24962-24971, doi:10.1074/jbc.M804358200.

Kupfer, R., S. Y. Liu, A. J. Allentoff, and J. A. Thompson, 2001, Comparisons of hydroperoxide isomerase and monooxygenase activities of cytochrome P450 for conversions of allylic hydroperoxides and alcohols to epoxyalcohols and diols: probing substrate reorientation in the active site: Biochemistry, v. 40, no. 38, p. 11490-11501.

Kveder, M., D. Merunka, M. Jokić, and B. Rakvin, 2008, Low-temperature electron-spin relaxation in the crystalline and glassy states of solid ethanol: Physical Review B, v. 77, no. 9, p. 094202, doi:10.1103/PhysRevB.77.094202.

Laskowski, R. A., and M. B. Swindells, 2011, LigPlot+: multiple ligand-protein interaction diagrams for drug discovery: Journal of Chemical Information and Modeling, v. 51, no. 10, p. 2778-2786, doi:10.1021/ci200227u.

Lee, D.-S., P. Nioche, Mats Hamberg, and C. S. Raman, 2008, Structural insights into the evolutionary paths of oxylipin biosynthetic enzymes: Nature, v. 455, no. 7211 , p. 363-368, doi:10.1038/nature07307.

Lee, D.-S., A. Yamada, H. Sugimoto, I. Matsunaga, H. Ogura, K. Ichihara, S. Adachi, S.-Y. Park, and Y. Shiro, 2003, Substrate Recognition and Molecular Mechanism of Fatty Acid Hydroxylation by Cytochrome P450 from Bacillus subtilis: Journal of Biological Chemistry, v. 278, no. 11, p. 9761 -9767, doi:10.1074/jbc.M211575200.

Léger, C., and P. Bertrand, 2008, Direct electrochemistry of redox enzymes as a tool for mechanistic studies: Chemical Reviews, v. 108, no. 7, p. 2379-2438, doi:10.1021/cr0680742.

Li, L., Z. Chang, Z. Pan, Z.-Q. Fu, and X. Wang, 2008, Modes of heme binding and substrate access for cytochrome P450 CYP74A revealed by crystal structures of allene oxide synthase: Proceedings of the National Academy of Sciences of the United States of America, v. 105, no. 37, p. 13883-13888, doi:10.1073/pnas.0804099105.

Li, H., and Thomas L. Poulos, 1999, Fatty acid metabolism, conformational change, and electron transfer in cytochrome P-450BM-3: Biochimica et Biophysica Acta (BBA) - Molecular and Cell Biology of Lipids, v. 1441, no. 2-3, p. 141-149, doi:10.1016/S1388-1981(99)00161-4.

Li, H., and Thomas L. Poulos, 1997, The structure of the cytochrome p450BM-3 haem domain complexed with the fatty acid substrate, palmitoleic acid: Nat Struct Mol Biol, v. 4, no. 2, p. 140-146, doi:10.1038/nsb0297-140. 
Liu, W., C. E. Rogge, B. Bambai, G. Palmer, A.-L. Tsai, and R. J. Kulmacz, 2004, Characterization of the heme environment in Arabidopsis thaliana fatty acid alpha-dioxygenase-1: The Journal of Biological Chemistry, v. 279, no. 28, p. 29805-29815, doi:10.1074/jbc.M401779200.

Lloyd, T., A. Krol, D. Campanaro, and M. Malkowski, 2006, Purification, crystallization and preliminary $X$-ray diffraction analysis of pathogen-inducible oxygenase (PIOX) from Oryza sativa: Acta Crystallographica. Section F, Structural Biology and Crystallization Communications, v. 62, no. Pt 4, p. 365-367, doi:10.1107/S1744309106007305.

Lottspeich, F., J. Engels, and O. Schiemann, 2006, Bioanalytik: München, Elsevier, 441-458 p.

Martinis, S. A., W. M. Atkins, P. S. Stayton, and S. G. Sligar, 1989, A conserved residue of cytochrome P-450 is involved in heme-oxygen stability and activation: J. Am. Chem. Soc., v. 111, no. 26, p. 92529253, doi:10.1021/ja00208a031.

Matsumura, H., M. Wakatabi, S. Omi, A. Ohtaki, N. Nakamura, M. Yohda, and H. Ohno, 2008, Modulation of Redox Potential and Alteration in Reactivity via the Peroxide Shunt Pathway by Mutation of Cytochrome P450 around the Proximal Heme Ligandt: Biochemistry, v. 47, no. 16, p. 4834-4842, doi:10.1021/bi800142v.

Matsunaga, I., A. Yamada, D.-S. Lee, E. Obayashi, N. Fujiwara, K. Kobayashi, H. Ogura, and Y. Shiro, 2002, Enzymatic Reaction of Hydrogen Peroxide-Dependent Peroxygenase Cytochrome P450s: Kinetic Deuterium Isotope Effects and Analyses by Resonance Raman Spectroscopy: Biochemistry, v. 41, no. 6, p. 1886-1892, doi:10.1021/bi011883p.

Mazur, P., K. Nakanishi, A. A. E. El-Zayat, and Sewell P. Champe, 1991, Structure and synthesis of sporogenic psi factors from Aspergillus nidulans: J. Chem. Soc., Chem. Commun., no. 20, p. 14861487, doi:10.1039/C39910001486.

McLean, K. J. et al., 2008, Characterization of active site structure in CYP121. A cytochrome P450 essential for viability of Mycobacterium tuberculosis H37Rv: The Journal of Biological Chemistry, v. 283, no. 48, p. 33406-33416, doi:10.1074/jbc.M802115200.

McLean, K. J., P. Lafite, C. Levy, M. R. Cheesman, N. Mast, I. A. Pikuleva, D. Leys, and Andrew W Munro, 2009, The Structure of Mycobacterium tuberculosis CYP125: molecular basis for cholesterol binding in a P450 needed for host infection: The Journal of Biological Chemistry, v. 284, no. 51, p. 35524-35533, doi:10.1074/jbc.M109.032706.

Mertens, H. D. T., and D. I. Svergun, 2010, Structural characterization of proteins and complexes using small-angle X-ray solution scattering: Journal of Structural Biology, v. 172, no. 1, p. 128-141, doi:10.1016/j.jsb.2010.06.012.

Michael Garavito, R., M. G. Malkowski, and D. L. DeWitt, 2002, The structures of prostaglandin endoperoxide $\mathrm{H}$ synthases-1 and -2: Prostaglandins \& Other Lipid Mediators, v. 68-69, no. 0, p. 129152, doi:10.1016/S0090-6980(02)00026-6.

Moon, A. F., G. A. Mueller, X. Zhong, and L. C. Pedersen, 2010, A synergistic approach to protein crystallization: combination of a fixed-arm carrier with surface entropy reduction: Protein Science: $A$ Publication of the Protein Society, v. 19, no. 5, p. 901-913, doi:10.1002/pro.368. 
Mukherjee, A., A. M. Angeles-Boza, G. S. Huff, and J. P. Roth, 2010, Catalytic Mechanism of a Heme and Tyrosyl Radical-Containing Fatty Acid $\alpha$-(Di)oxygenase: J. Am. Chem. Soc., v. 133, no. 2, p. $227-$ 238, doi:10.1021/ja104180v.

Mukherjee, A., D. W. Brinkley, K.-M. Chang, and J. P. Roth, 2007, Molecular Oxygen Dependent Steps in Fatty Acid Oxidation by Cyclooxygenase-1+: Biochemistry, v. 46, no. 13, p. 3975-3989, doi:10.1021/bi602502j.

Nelson, M. J., B. A. Brennan, D. B. Chase, R. A. Cowling, G. N. Grove, and R. C. Scarrow, 1995, Structure and kinetics of formation of catechol complexes of ferric soybean lipoxygenase-1: Biochemistry, v. 34, no. 46, p. 15219-15229.

Nilsson, T., E. Martínez, A. Manresa, and Ernst H. Oliw, 2010, Liquid chromatography/tandem mass spectrometric analysis of 7,10-dihydroxyoctadecenoic acid, its isotopomers, and other 7,10dihydroxy fatty acids formed by Pseudomonas aeruginosa 42A2: Rapid Communications in Mass Spectrometry, v. 24, no. 6, p. 777-783, doi:10.1002/rcm.4446.

Northrop, D. B., 1975, Steady-state analysis of kinetic isotope effects in enzymic reactions: Biochemistry, v. 14, no. 12, p. 2644-2651.

Oliw, E H, C Su, T. Skogström, and G. Benthin, 1998, Analysis of novel hydroperoxides and other metabolites of oleic, linoleic, and linolenic acids by liquid chromatography-mass spectrometry with ion trap MSn: Lipids, v. 33, no. 9, p. 843-852.

Oliw, Ernst H, U. Garscha, T. Nilsson, and M. Cristea, 2006, Payne rearrangement during analysis of epoxyalcohols of linoleic and alpha-linolenic acids by normal phase liquid chromatography with tandem mass spectrometry: Analytical Biochemistry, v. 354, no. 1, p. 111-126, doi:10.1016/j.ab.2006.04.010.

Oliw, Ernst H, F. Jernerén, I. Hoffmann, M. Sahlin, and U. Garscha, 2010, Manganese lipoxygenase oxidizes bis-allylic hydroperoxides and octadecenoic acids by different mechanisms: Biochimica Et Biophysica Acta, doi:10.1016/j.bbalip.2010.12.002.

Oliw, Ernst H, A. Wennman, I. Hoffmann, U. Garscha, Mats Hamberg, and F. Jernerén, 2011, Stereoselective oxidation of regioisomeric octadecenoic acids by fatty acid dioxygenases: Journal of Lipid Research, v. 52, no. 11, p. 1995-2004, doi:10.1194/jlr.M018259.

Ortiz de Montellano, P. R., 2010, Hydrocarbon Hydroxylation by Cytochrome P450 Enzymes: Chemical reviews, v. 110, no. 2, p. 932, doi:10.1021/cr9002193.

Pannier, M., S. Veit, A Godt, G Jeschke, and H. . Spiess, 2000, Dead-Time Free Measurement of Dipole-Dipole Interactions between Electron Spins: Journal of Magnetic Resonance, v. 142, no. 2, p. 331-340, doi:10.1006/jmre.1999.1944.

Peterson, J. A., and S. E. Graham, 1998, A close family resemblance: the importance of structure in understanding cytochromes P450: Structure, v. 6, p. 1079-1085, doi:10.1016/S0969-2126(98)001099. 
Petoukhov, M. V., and D. I. Svergun, 2005, Global Rigid Body Modeling of Macromolecular Complexes against Small-Angle Scattering Data: Biophysical Journal, v. 89, no. 2, p. 1237-1250, doi:10.1529/biophysj.105.064154.

Pochapsky, T. C., S. Kazanis, and M. Dang, 2010, Conformational plasticity and structure/function relationships in cytochromes P450: Antioxidants \& Redox Signaling, v. 13, no. 8, p. 1273-1296, doi:10.1089/ars.2010.3109.

Poulos, Thomas L., 1996, The role of the proximal ligand in heme enzymes: Journal of Biological Inorganic Chemistry, v. 1, no. 4, p. 356-359, doi:10.1007/s007750050064.

Putnam, C. D., M. Hammel, G. L. Hura, and J. A. Tainer, 2007, X-Ray Solution Scattering (SAXS) Combined with Crystallography and Computation: Defining Accurate Macromolecular Structures, Conformations and Assemblies in Solution: Quarterly Reviews of Biophysics, v. 40, no. 03, p. 191285, doi:10.1017/S0033583507004635.

Ranaghan, K. E., and A. J. Mulholland, 2010, Computer simulations of quantum tunnelling in enzymecatalysed hydrogen transfer reactions: Interdisciplinary Sciences, Computational Life Sciences, v. 2, no. 1, p. 78-97, doi:10.1007/s12539-010-0093-y.

Reginsson, G. W., and O. Schiemann, 2011, Pulsed electron-electron double resonance: beyond nanometre distance measurements on biomacromolecules: Biochemical Journal, v. 434, no. 3, p. 353-363, doi:10.1042/BJ20101871.

Reverberi, M., F. Punelli, M. Scarpari, E. Camera, S. Zjalic, A. Ricelli, Corrado Fanelli, and Anna Adele Fabbri, 2010, Lipoperoxidation affects ochratoxin A biosynthesis in Aspergillus ochraceus and its interaction with wheat seeds: Applied Microbiology and Biotechnology, v. 85, no. 6, p. 1935-1946, doi:10.1007/s00253-009-2220-4.

Rickert, K. W., and J. P. Klinman, 1999, Nature of Hydrogen Transfer in Soybean Lipoxygenase 1: Separation of Primary and Secondary Isotope Effectst: Biochemistry, v. 38, no. 38, p. 12218-12228, doi:10.1021/bi990834y.

Rieke, C. J., A. M. Mulichak, R. M. Garavito, and W. L. Smith, 1999, The Role of Arginine 120 of Human Prostaglandin Endoperoxide H Synthase-2 in the Interaction with Fatty Acid Substrates and Inhibitors: Journal of Biological Chemistry, v. 274, no. 24, p. 17109 -17114, doi:10.1074/jbc.274.24.17109.

Rittle, J., and M. T. Green, 2010, Cytochrome P450 Compound I: Capture, Characterization, and C-H Bond Activation Kinetics: Science, v. 330, no. 6006, p. 933 -937, doi:10.1126/science.1193478.

Rogge, C. E., B. Ho, W. Liu, R. J. Kulmacz, and A.-L. Tsai, 2006, Role of Tyr348 in Tyr385 Radical Dynamics and Cyclooxygenase Inhibitor Interactions in Prostaglandin H Synthase-2: Biochemistry, $\mathrm{V}$. 45, no. 2, p. 523-532, doi:10.1021/bi051235w.

Rouzer, C. A., and Lawrence J. Marnett, 2003, Mechanism of Free Radical Oxygenation of Polyunsaturated Fatty Acids by Cyclooxygenases: Chem. Rev., v. 103, no. 6, p. 2239-2304, doi:10.1021/cr000068x. 
Roy, A., A. Kucukural, and Yang Zhang, 2010, I-TASSER: a unified platform for automated protein structure and function prediction: Nature Protocols, v. 5, no. 4, p. 725-738, doi:10.1038/nprot.2010.5.

Schneider, C., D. A. Pratt, N. A. Porter, and A. R. Brash, 2007, Control of oxygenation in lipoxygenase and cyclooxygenase catalysis: Chemistry \& Biology, v. 14, no. 5, p. 473-488, doi:10.1016/j.chembiol.2007.04.007.

Seifert, A., and J. Pleiss, 2009, Identification of selectivity-determining residues in cytochrome P450 monooxygenases: a systematic analysis of the substrate recognition site 5: Proteins, v. 74 , no. 4, p. 1028-1035, doi:10.1002/prot.22242.

Sharma, S. C., and J. P. Klinman, 2008, Experimental Evidence for Hydrogen Tunneling when the Isotopic Arrhenius Prefactor (AH/AD) is Unity: Journal of the American Chemical Society, v. 130, no. 52, p. 17632-17633, doi:10.1021/ja806354w.

Sharma, P. K., S. P. de Visser, and S. Shaik, 2003, Can a Single Oxidant with Two Spin States Masquerade as Two Different Oxidants? A Study of the Sulfoxidation Mechanism by Cytochrome P450: J. Am. Chem. Soc., v. 125, no. 29, p. 8698-8699, doi:10.1021/ja035135u.

Sidhu, R. S., J. Y. Lee, C. Yuan, and W. L. Smith, 2010, Comparison of Cyclooxygenase-1 Crystal Structures: Cross-Talk between Monomers Comprising Cyclooxygenase-1 Homodimers,: Biochemistry, v. 49, no. 33, p. 7069-7079, doi:10.1021/bi1003298.

Simmons, D. L., R. M. Botting, and T. Hla, 2004, Cyclooxygenase Isozymes: The Biology of Prostaglandin Synthesis and Inhibition: Pharmacological Reviews, v. 56, no. 3, p. 387-437, doi:10.1124/pr.56.3.3.

Sirim, D., M. Widmann, F. Wagner, and J. Pleiss, 2010, Prediction and analysis of the modular structure of cytochrome P450 monooxygenases: BMC Structural Biology, v. 10, p. 34, doi:10.1186/1472-6807-10-34.

Smyth, D. R., M. K. Mrozkiewicz, W. J. McGrath, P. Listwan, and B. Kobe, 2003, Crystal structures of fusion proteins with large-affinity tags: Protein Science: A Publication of the Protein Society, v. 12, no. 7, p. 1313-1322, doi:10.1110/ps.0243403.

Spivey, H. O., and J. Ovádi, 1999, Substrate Channeling: Methods, v. 19, no. 2, p. 306-321, doi:10.1006/meth.1999.0858.

Stryer, L., 2007, Biochemie: Heidelberg, Elsevier, Spektrum Akademischer Verlag.

Su, Chao, and Ernst H. Oliw, 1996, Purification and Characterization of Linoleate 8-Dioxygenase from the Fungus Gaeumannomyces graminis as a Novel Hemoprotein: Journal of Biological Chemistry, v. 271 , no. 24 , p. $14112-14118$, doi:10.1074/jbc.271.24.14112.

Su, Chao, M. Sahlin, and Ernst H. Oliw, 1998, A Protein Radical and Ferryl Intermediates Are Generated by Linoleate Diol Synthase, a Ferric Hemeprotein with Dioxygenase and Hydroperoxide Isomerase Activities: Journal of Biological Chemistry, v. 273, no. 33, p. $20744-20751$, doi:10.1074/jbc.273.33.20744. 
Thatcher, L. F., J. M. Manners, and K. Kazan, 2009, Fusarium oxysporum hijacks COI1-mediated jasmonate signaling to promote disease development in Arabidopsis: The Plant Journal, v. 58, no. 6, p. 927-939, doi:10.1111/j.1365-313X.2009.03831.x.

Thuresson, E. D., K. M. Lakkides, C. J. Rieke, Y. Sun, B. A. Wingerd, R. Micielli, A. M. Mulichak, M. G. Malkowski, R. M. Garavito, and W. L. Smith, 2001, Prostaglandin Endoperoxide H Synthase-1: Journal of Biological Chemistry, v. 276, no. 13, p. 10347 -10357, doi:10.1074/jbc.M009377200.

Tsai, A.-L., and R. J. Kulmacz, 2010, Prostaglandin H synthase: resolved and unresolved mechanistic issues: Archives of Biochemistry and Biophysics, v. 493, no. 1, p. 103-124, doi:10.1016/j.abb.2009.08.019.

Tsitsigiannis, D. I., J.-W. Bok, D. Andes, K. F. Nielsen, J. C. Frisvad, and Nancy P Keller, 2005, Aspergillus cyclooxygenase-like enzymes are associated with prostaglandin production and virulence: Infection and Immunity, v. 73, no. 8, p. 4548-4559, doi:10.1128/IAI.73.8.4548-4559.2005.

Tsitsigiannis, D. I., and Nancy P. Keller, 2006, Oxylipins act as determinants of natural product biosynthesis and seed colonization in Aspergillus nidulans: Molecular Microbiology, v. 59, no. 3, p. 882-892, doi:10.1111/j.1365-2958.2005.05000.x.

Tsitsigiannis, D. I., T. M. Kowieski, R. Zarnowski, and Nancy P Keller, 2004, Endogenous lipogenic regulators of spore balance in Aspergillus nidulans: Eukaryotic Cell, v. 3, no. 6, p. 1398-1411, doi:10.1128/EC.3.6.1398-1411.2004.

Tsitsigiannis, D. I., T. M. Kowieski, R. Zarnowski, and Nancy P Keller, 2005, Three putative oxylipin biosynthetic genes integrate sexual and asexual development in Aspergillus nidulans: Microbiology (Reading, England), v. 151, no. Pt 6, p. 1809-1821, doi:10.1099/mic.0.27880-0.

Tsitsigiannis, D. I., R. Zarnowski, and Nancy P Keller, 2004, The lipid body protein, PpoA, coordinates sexual and asexual sporulation in Aspergillus nidulans: The Journal of Biological Chemistry, v. 279, no. 12, p. 11344-11353, doi:10.1074/jbc.M310840200.

Udit, A. K., M. G. Hill, and H. B. Gray, 2006, Electrochemistry of Cytochrome P450 BM3 in Sodium Dodecyl Sulfate Films: Langmuir : the ACS journal of surfaces and colloids, v. 22, no. 25, p. 1085410857, doi:10.1021/la061162x.

Vecchio, A. J., B. J. Orlando, R. Nandagiri, and M. G. Malkowski, 2012, Investigating Substrate Promiscuity in Cyclooxygenase-2 THE ROLE OF ARG-120 AND RESIDUES LINING THE HYDROPHOBIC GROOVE: Journal of Biological Chemistry, v. 287, no. 29, p. 24619-24630, doi:10.1074/jbc.M112.372243.

Vergopoulou, S., D. Galanopoulou, and P. Markaki, 2001, Methyl jasmonate stimulates aflatoxin B1 biosynthesis by Aspergillus parasiticus: Journal of Agricultural and Food Chemistry, v. 49, no. 7, p. 3494-3498.

Verhagen, M. F. J. M., E. T. M. Meussen, and W. R. Hagen, 1995, On the reduction potentials of Fe and $\mathrm{Cu}$ ? Zn containing superoxide dismutases: Biochimica et Biophysica Acta (BBA) - General Subjects, v. 1244, no. 1, p. 99-103, doi:10.1016/0304-4165(94)00201-8. 
Wasternack, C., 2007, Jasmonates: an update on biosynthesis, signal transduction and action in plant stress response, growth and development: Annals of Botany, v. 100, no. 4, p. 681-697, doi:10.1093/aob/mcm079.

Werck-Reichhart, D., and R. Feyereisen, 2000, Cytochromes P450: a success story: Genome Biology, v. 1 , no. 6 , p. REVIEWS3003.

Wernimont, A., and A. Edwards, 2009, In situ proteolysis to generate crystals for structure determination: an update: PloS One, v. 4, no. 4, p. e5094, doi:10.1371/journal.pone.0005094.

Witherow, L. E., and J. B. Houston, 1999, Sigmoidal kinetics of CYP3A substrates: an approach for scaling dextromethorphan metabolism in hepatic microsomes and isolated hepatocytes to predict in vivo clearance in rat: The Journal of Pharmacology and Experimental Therapeutics, v. 290, no. 1, p. 58-65.

Wu, G., J.-M. Lü, W. A. van der Donk, R. J. Kulmacz, and A. Tsai, 2011, Cyclooxygenase reaction mechanism of prostaglandin $\mathrm{H}$ synthase from deuterium kinetic isotope effects: Journal of Inorganic Biochemistry, v. 105, no. 3, p. 382-390, doi:16/j.jinorgbio.2010.11.015.

Wu, G., C. E. Rogge, J.-S. Wang, R. J. Kulmacz, G. Palmer, and A.-L. Tsai, 2007, Oxyferryl Heme and Not Tyrosyl Radical Is the Likely Culprit in Prostaglandin H Synthase-1 Peroxidase Inactivation: Biochemistry, v. 46, no. 2, p. 534-542, doi:10.1021/bi061859h.

Yeh, H.-C., P.-Y. Hsu, J.-S. Wang, A.-L. Tsai, and L.-H. Wang, 2005, Characterization of heme environment and mechanism of peroxide bond cleavage in human prostacyclin synthase: Biochimica et biophysica acta, v. 1738, no. 1-3, p. 121-132, doi:10.1016/j.bbalip.2005.11.007.

Yuan, C., C. J. Rieke, G. Rimon, B. A. Wingerd, and W. L. Smith, 2006, Partnering between monomers of cyclooxygenase-2 homodimers: Proceedings of the National Academy of Sciences, v. 103, no. 16, p. $6142-6147$, doi:10.1073/pnas.0601805103.

Zhang, Yang, 2008a, I-TASSER server for protein 3D structure prediction: BMC Bioinformatics, v. 9, p. 40, doi:10.1186/1471-2105-9-40.

Zhang, Yang, 2008b, Progress and challenges in protein structure prediction: Current opinion in structural biology, v. 18, no. 3, p. 342-348, doi:10.1016/j.sbi.2008.02.004.

Zwanzig, R., A. Szabo, and B. Bagchi, 1992, Levinthal's paradox.: Proceedings of the National Academy of Sciences of the United States of America, v. 89, no. 1, p. 20-22. 


\section{Parts of this thesis have been published:}

1 Nadler A, Koch C, Brodhun F, Wehland J-D, Tittmann K, Feußner I \& Diederichsen U (2011) Influence of substrate dideuteration on the reaction of the bifunctional heme enzyme psi factor producing oxygenase A (PpoA). Chembiochem 12, 728-737

2 Koch C, Fielding AJ, Brodhun F, Bennati M \& Feußner I (2012) Linoleic acid positioning in psi factor producing oxygenase $A$, a fusion protein with an atypical cytochrome P450 activity. FEBS Journal 279, 1594-1606 


\section{ABBREVIATIONS}

Å- Angstrom, $10^{-10} \mathrm{~m}$

A- Deoxyadenosine

Ala- Alanine

$\alpha$-DOX- Alpha dioxygenase

AOS- Allene oxide synthase

APS- Ammonium persulfate

Arg- Arginine

Asn- Asparagine

a.u.- Arbitrary units

bp- Base pair

${ }^{\circ} \mathrm{C}$ - Degree Celsius

C- Deoxycytidine

$\mathrm{C}_{5}$ - $\mathrm{d}_{2}$-OA- (9Z) 5,5-Dideutero-octadec-9-enoic acid

$\mathrm{C}_{8}$ - $\mathrm{d}_{2}$-OA- (9Z) 8,8-Dideutero-octadec-9-enoic acid

$\mathrm{CW}$ - Continuous wave

Cys- Cysteine

Da- Dalton

DEER- Double electron electron resonance

$\mathrm{diH}_{2} \mathrm{O}$ - Deionized water

DiHODE- (9Z, 12Z) Dihydroxy-octadeca-9,12-dienoic acid

DiHOME- (9Z) Dihydroxy-octadec-9-enoic acid

DMSO- Dimethyl sulfoxide

DNA- Deoxyribonucleic acid

dNTP- Deoxyribonucleotide triphosphate

$\mathrm{D}_{2} \mathrm{O}$ - Deuterium oxide

DOX- Dioxygenase

DTT- Dithiothreitol

E- Glutamic acid

EDTA- Ethylenediaminetetraacetic acid

EM- electron microscopy

EPR- Electron paramagnetic resonance

ESI- Electrospray ionisation

$\mathrm{Et}_{2} \mathrm{O}$ - Diethyl ether

EtOH- Ethanol

F- Phenylalanine

G- Deoxyguanosine

g- Gram 
Abbreviations

Gln- Glutamine

Glu- Glutamic acid

GraFix- Gradient Fixation

h- Hours

$\mathrm{H}$ - Histidine

HAc- Acetic acid

$\mathrm{HCl}$ - hydrochloric acid

His- Histidine

HEPES- 4-(2-hydroxyethyl)-1-piperazine-ethanesulfonicacid

HODE- (9Z, 12Z) Hydroxy-octadeca-9,12-dienoic acid

HOME- (9Z) Hydroxy-octadec-9-enoic acid

HPLC- High performance liquid chromatography

HPODE- $(9 Z, 12 Z)$ Hydroperoxy-octadeca-9,12-dienoic acid

HPOME- (9Z) Hydroperoxy-octadec-9-enoic acid

HR- High resolution

IPTG-IsopropyI- $\beta$-D-thiogalaktoside

IUPAC- International Union of Pure and Applied Chemistry

$\mathrm{k}_{\mathrm{D}}$ - Dissociation constant

KIE- Kinetic isotope effect

KODE- $(9 Z, 12 Z)$ Oxo-octadeca-9,12-dienoic acid

KOME- (9Z) Oxo-octadec-9-enoic acid

I- Liter

L- Leucine

$\lambda$ - Wavelength

LDS- Linoleate diol synthase

Leu- Leucine

LOX- Lipoxygenase

M- Molar (mole per liter)

$\mathrm{m}$ - Meter

mA- Milliampere

MeCN-Acetonitrile

Met- Methionine

MetOH- Methanol

MPI- Max Planck Institute

MS- Mass spectrometry

MW- Molecular weight

$m / z$ - Ratio of mass to charge

$\mathrm{N}$ - Asparagine

$O D_{X^{-}}$Optical density at a wavelength of $X \mathrm{~nm}$

P450- Cytochrome P450 
PCR- Polymerase chain reaction

PDB- Protein data base (http://www.rcsb.org/pdb/home/home.do)

PELDOR- Pulsed electron double resonance

PGIS- Prostacyclin synthase

PGHSx- Prostaglandin $\mathrm{H}_{2}$ synthase, isoform $\mathrm{x}$

$\mathrm{pH}$ - The negative decadic logarithm of hydrogen- ion activity $\left[-\log _{10}\left[\mathrm{H}_{3} \mathrm{O}\right]^{+}\right.$

Phe- Phenylalanine

PIPES- Piperazine- N,N'- bis- ethanesulfonic acid

PPIX- Protoporphyrin IX

Ppo- Psi- factor producing oxygenase

Psi- Precocious sexual inducer

Q- Glutamine

R- Arginine

RMSD- Root- mean- square deviation

rpm- Rotations per minute

RP- Reverse phase

RT- Room temperature

s- Second

SAXS- Small-angle $X$ - ray scattering

SDS- Sodium dodecyl sulfate

SRS- Substrate recognition site (of cytochrome P450s)

T- Deoxythymidine

$\mathrm{T}_{1}$ - Spin- lattice relaxation

$\mathrm{T}_{2}$ - Spin- spin relaxation

$\mathrm{T}_{\mathrm{m}}$ - Phase memory time

TEMED- $N, N, N^{\prime}, N^{\prime}$ - Tetramethylethylenediamine

TFA- Trifluoroacetic acid

Tris- 2- Amino- 2- hydroxymethyl- propane- 1,3- diol

Trp- Tryptophane

Tyr- Tyrosine

U- Unit (unit of enzyme activity)

UV- Ultra violet

vis- Visible

V- Volt

Val- Valine

v/v- Volume per volume

W- Tryptophane

Wt- Wild type

w/v- Weight per volume

$Y$ - Tyrosine 
Abbreviations 


\section{CURRICULUM VITAE}

\section{Personal Information}

Surname, Name

Date/Place of birth

Nationality
Koch, Christian

$10^{\text {th }}$ November $1984 /$ Muehlhausen

German

\section{Education}

Georg-August-University Goettingen

Doctoral thesis

January 2010 to September 2012

Structure and Function of A.nidulans Psi factor producing oxygenase $A$

Group of Prof. Dr. Ivo Feußner

Scholarship holder of the DFG-IRTG1422 (01.10-09.12)

University of Applied science Jena

Dipl.-Ing. (FH)

Effective recombinant protein production in Pichia pastoris

February 2007 to October 2007 through multi copy integration of the heterologous gene c-LECTA GmbH, Leipzig

University of Applied science Jena

Pharma-Biotechnology

Seilergymnasium Schlotheim

Abitur

August 1995 to June 2003

October 2003 to October 2007 